\title{
SPATIAL VARIABILITY OF SOIL VELOCITY USING PASSIVE SURFACE WAVE TESTING
}

\author{
A Thesis \\ presented to \\ the Faculty of California Polytechnic State University, \\ San Luis Obispo
}

\author{
In Partial Fulfillment \\ of the Requirements for the Degree \\ Master of Science in Civil and Environmental Engineering
}

by

Daniel Raymond Wagstaffe

December 2015 
(C) 2015

Daniel Raymond Wagstaffe

ALL RIGHTS RESERVED 
COMMITTEE MEMBERSHIP

TITLE:

Spatial Variability of Soil Velocity using

Passive Surface Wave Testing

AUTHOR: Daniel Raymond Wagstaffe

DATE SUBMITTED: December 2015

COMMITTEE CHAIR: $\quad$ Robb Moss, Ph.D., PE

Associate Professor of Civil Engineering

COMMITTEE MEMBER: John Jasbinsek, Ph.D.

Associate Professor of Physics

COMMITTEE MEMBER: Gregg Fiegel, Ph.D., PE, GE

Professor of Civil Engineering

COMMITTEE MEMBER: Dmitry Maleev, Ph.D.

Candidate of Geology and Mineralogy

Associate Professor at Far Eastern State

Transportation University 


\section{ABSTRACT \\ Spatial Variability of Soil Velocity using Passive Surface Wave Testing \\ Daniel Raymond Wagstaffe}

Lifelines such as highways, pipelines, telecommunication lines, and powerlines provide communities with vital services, and their functionality is dependent upon the soil that supports them. However, when designing the infrastructure, it can be difficult to know where to test the soil in order to give spatially representative sampling, particularly for long, lifeline structures. Finding this distance requires knowledge of the spatial correlation and/or the spatial variability of the soil parameter (stiffness, cohesion, etc.). But this correlation distance is not typically found in practice because it requires large amounts of data and the costs of retrieving that data can be high. Lack of representative sampling can lead to an overly conservative design but too much sampling can create an overly expensive sampling program. In this study, multiple tests using the geophysical method of spatial autocorrelation (SPAC) were conducted to find soil velocity along a 310 meter long profile. SPAC records passive surface waves which sample the underlying soil, and these surface waves can be used to create a shear wave velocity profile of the site. The spatial continuity of the stiffness (the soil velocity values) was then found using geostatistics. The geostastical tool primarily used in this study was the (semi-)variogram, but the covariance function and the correlogram are also shown. The distance that the soil parameter is minimally correlated with itself is assumed to be the maximum distance that gives representative sampling. This study found this distance (the range of the semi-variogram) to be 70 meters for 5 meters depth, 100 meters for 10 to 15 meters depth, and 90 meters for 30 meters depth. 


\section{ACKNOWLEDGMENTS}

First and foremost, I would like to thank Dr. Robb Moss. This study would not have been possible without him and I very much appreciate all the help he gave me. I always got undivided attention when I came in for advice and I never got shooed out the door. I am grateful that he not only helped develop this study, but also helped grow me as a student and an engineer.

My other committee members who each played a role in the development of this thesis. To Dr. John Jasbinsek who was eager to send reference material, enthusiastic to sit with me and discuss my newest questions, and willing even to put on gloves and help me collect the data. To Dr. Dmitry Maleev who was ready to give advice and guidance even before his office was set up and for the much needed help in translating the Russian code book. And to Dr. Gregg Fiegel who developed my engineering skills in previous classes, and who took the time to read through and constructively critic this thesis.

To my friends and roommates for working alongside me, for encouraging me, and for always providing the smile I needed (and the food I needed after long nights in the lab). To Tyler, Jordan, Luke, Rory, Nick, David, Riley, and Ballard: thank you.

And, of course, to my family. For pushing me and loving me since I was too young to know what engineering was and when I thought that a thesis defense had something to do with a dragon and a sword. I am the man I am today because of your love and guidance. Thank you. 


\section{TABLE OF CONTENTS}

Page

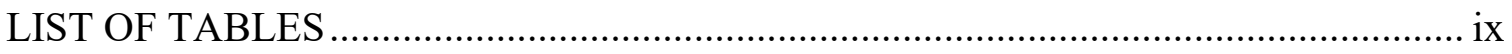

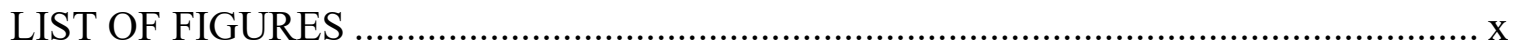

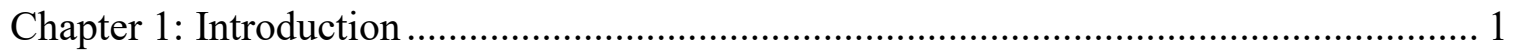

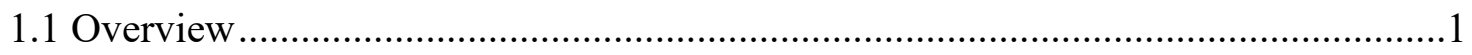

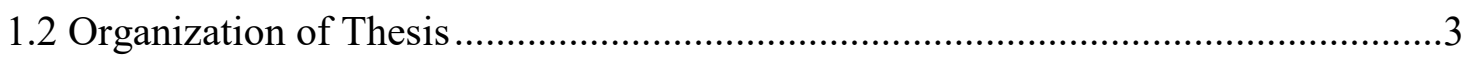

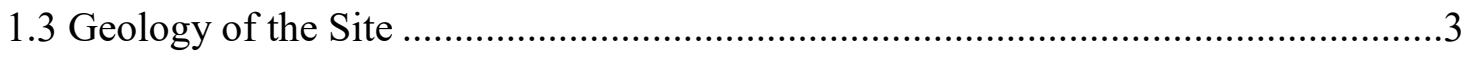

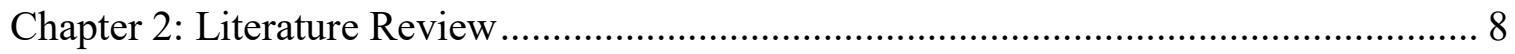

2.1 Review of the Spatial Autocorrelation (SPAC) Method............................................

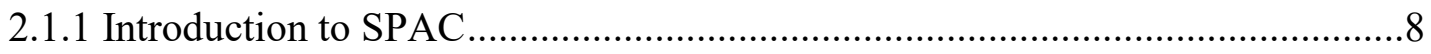

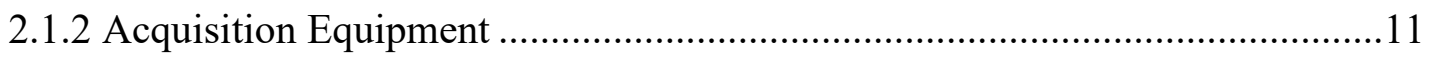

2.1.3 Surface Wave Measurement and Analysis ...................................................13

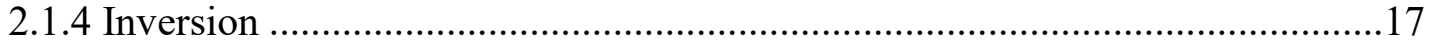

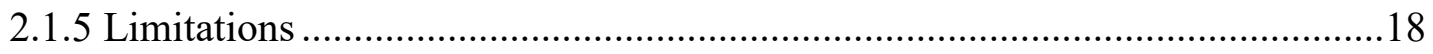

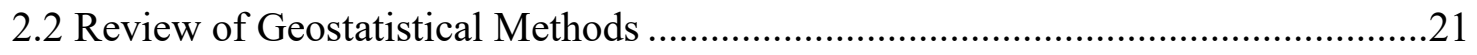

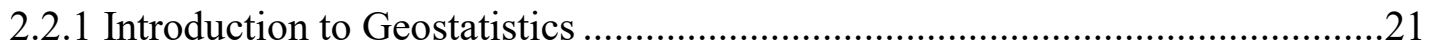

2.2.2 Spatial Continuity Functions ………………................................................24 


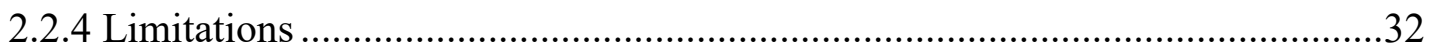

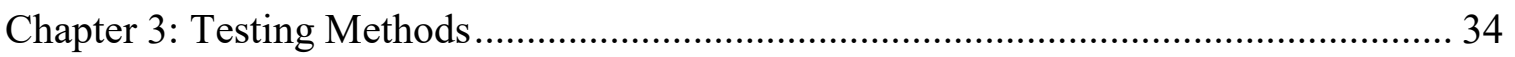

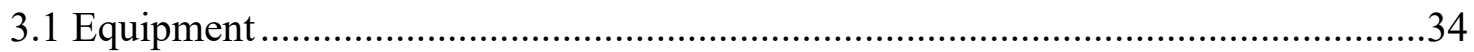

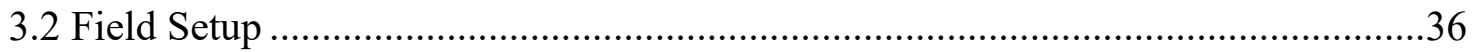

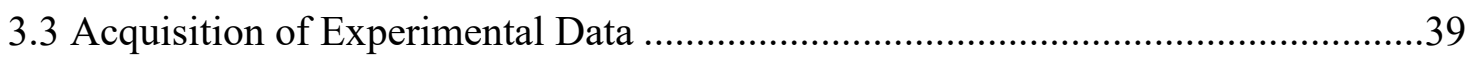

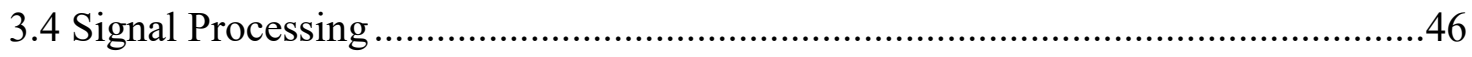

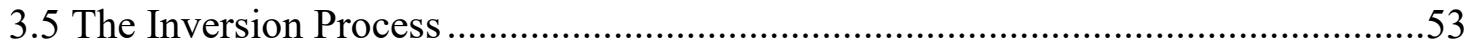

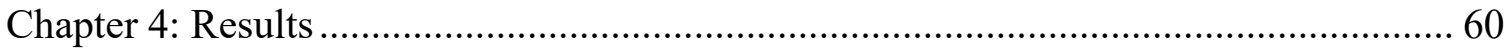

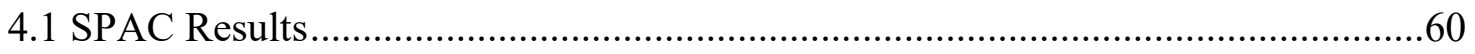

4.2 Empirical Semi-Variograms and Covariance Functions...........................................64

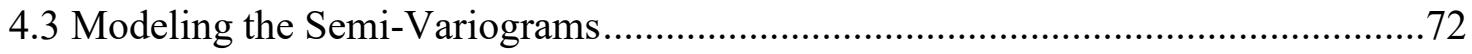

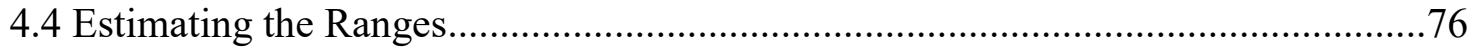

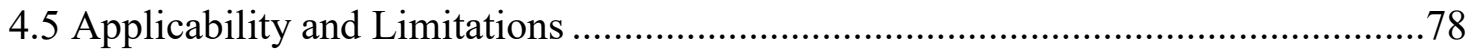

4.5.1 Applying Range Values to Other Sites ........................................................78

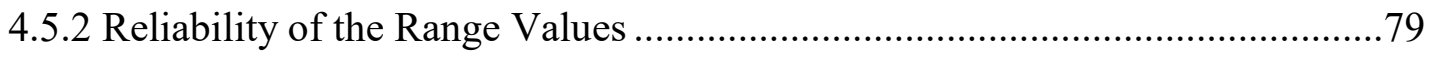

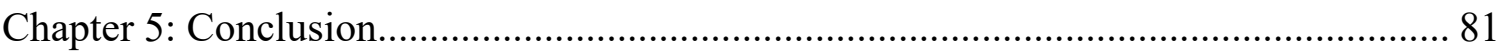

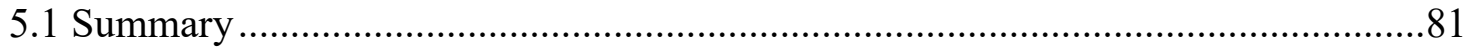




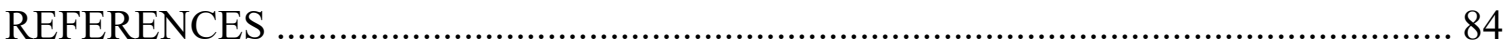

\section{APPENDICES}

Appendix A: Earth Systems Pacific's Boring Logs for the Cuesta College Sewer

Line and Pipe Bridge Replacement Project (2010)...............................................8

Appendix B: SPAC Results for Each Survey .....................................................97

Appendix C: Time weighted average Phase Velocity Values for Each Survey ..........127

Appendix D: MATALB Output: Semi-variograms, Covariance Functions, and

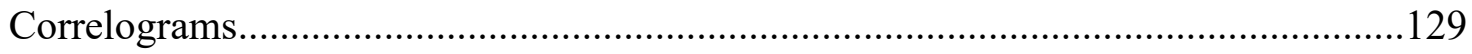

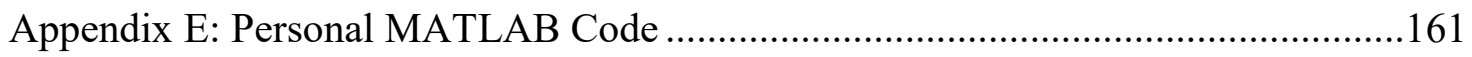




\section{LIST OF TABLES}

Table

Page

Table 1.1 Typical design for bandwidth, sample spacing, and sample depth according

to Russian code

Table 3.1 Shear wave velocity characteristics of geologic units in CA from Wills and

Clahan

Table 4.1 Coefficient of determination values for each data type and semi-variogram

model...

Table 4.2 Semi-variogram ranges from the spherical and Gaussian models for each

data type

Table 4.3 Summary of range values whose model has $R^{2}$ values greater than $50 \%$

and whose sill is visible within the data. 76

Table 4.4 Range values using all available data and averaging the values from 10

and $15 \mathrm{~m}$ depth 


\section{LIST OF FIGURES}

Figure

Page

Figure 1.1 Vicinity map of the site

4

Figure 1.2 Geologic map of the site and the surrounding area (California Conservation and California Geologic Survey, 2010) ................................................................... 5

Figure 1.3 Cross section of nearby location (provided by Earth Systems Pacific)............ 7

Figure 2.1 The derivation of spatial autocorrelation as presented by Kiochi Hayashi;

(a) Waves propagating in different directions, (b) the coherence of the wave propagation between pairs of receivers, and (c) averaging the coherence between all

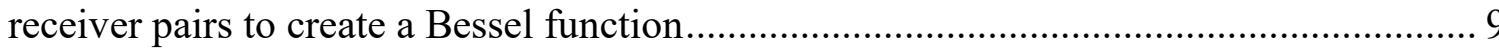

Figure 2.2 Geophone visual; (a) $4.5 \mathrm{~Hz}$ geophone used in this study and (b) crosssection of a typical moving coil type geophone (Foti et al., 2014, p. 193).................... 12 Figure 2.3 Illustration showing how shorter wavelengths (left) sample shallower material and longer wavelengths (right) sample deeper material 13

Figure 2.4 Example dispersion curve from the Geogiga Surface Plus (2012) manual.... 15

Figure 2.5 An example of how dispersion curves change with differing modes (Foti et al., 2014, p. 75); Each curve represents a single mode of propagation...... 16

Figure 2.6 Modal and apparent Rayleigh dispersion curves for normally dispersive (left) and inversely dispersive (right) material (Lai, 2005, p. 116) 17

Figure 2.7 Standard deviations for Vs models that only differ by number of layers;

(a) five-layer model and (b) ten-layer model (Foti et al., 2014, p. 347)..... 20 
Figure 2.8 Example h-scatterplot; (a) values along a line with 5 unit spacing, and

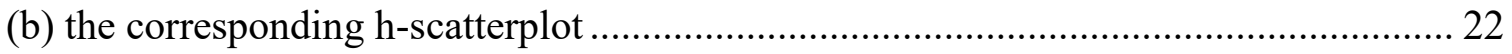

Figure 2.9 Example of how the correlation coefficient varies with lag distance............. 23

Figure 2.10 Example of how the moment of inertia varies with lag distance ................. 24

Figure 2.11 Typical shape of the semi-variogram and covariance function................... 26

Figure 2.12 Basic transition models........................................................................ 29

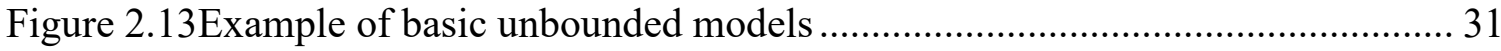

Figure 2.14 Deciding on a model from the first few points in the semi-variogram ......... 32

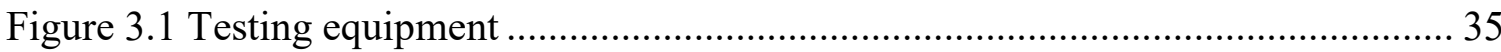

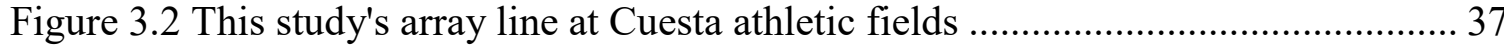

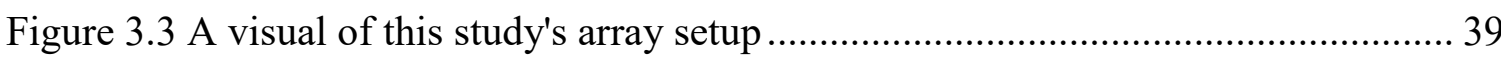

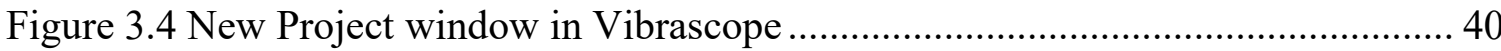

Figure 3.5 Configuration window on the Acquisition tab .......................................... 41

Figure 3.6 The Configuration window if "Advanced Mode" is not selected................... 42

Figure 3.7 An example of an appropriate 10-second recording................................... 43

Figure 3.8 Vibrascope's indication of how long the recording has been receiving data... 44

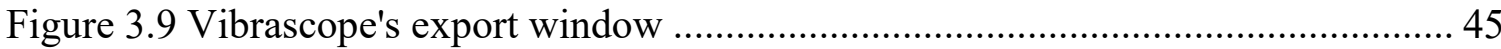

Figure 3.10 Importing seismic data in Geogiga Seismic Pro....................................... 47

Figure 3.11 Setting geometry in Geogiga Seismic Pro ............................................ 47

Figure 3.12 Text file with receiver locations ......................................................... 48

Figure 3.13 Dispersion settings in Geogiga Seismic Pro .......................................... 49 
Figure 3.14 Spectra combination in Geogiga Seismic Pro to combine 3 dispersion

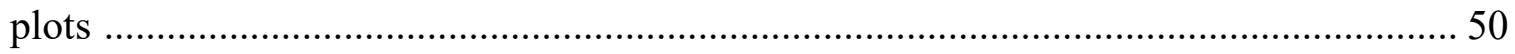

Figure 3.15 Trace, H/V, and dispersion curve plots in Geogiga Seismic Pro .................. 52

Figure 3.16 Saving an image in Geogiga Seismic Pro.................................................... 53

Figure 3.17 (a) Poor dispersion curve picking, and (b) appropriate dispersion curve

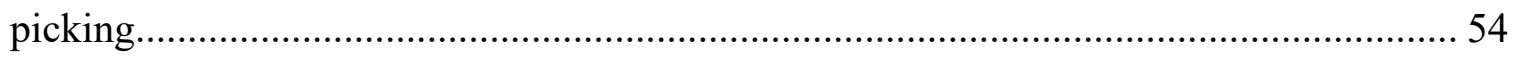

Figure 3.18 Allowing the inversion process to vary by velocity and depth ..................... 55

Figure 3.19 The shear wave velocity profile, dispersion curve, and error iteration

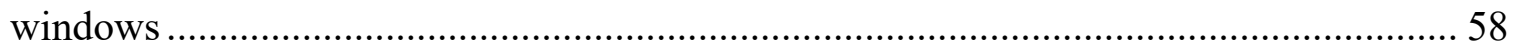

Figure 4.1 Shear wave velocity profile for each survey with dotted lines at analyzed

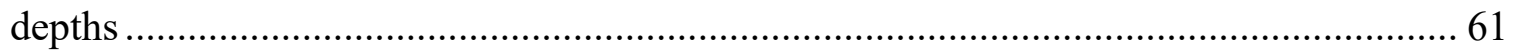

Figure 4.2 Example of Rayleigh wave and shear wave velocity profiles from

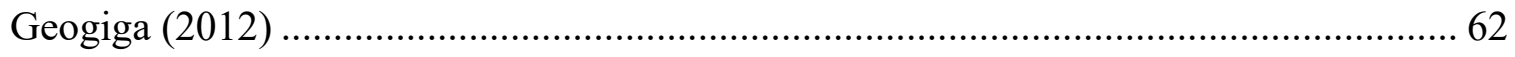

Figure 4.3 Time-averaged Rayleigh wave velocity across the site................................... 63

Figure 4.4 Time-averaged shear wave velocity across the site......................................... 64

Figure 4.5 Semi-variogram and covariance function for time weighted average

Rayleigh wave velocity using all available data points ....................................................6 66

Figure 4.6 Time weighted average Rayleigh wave velocity fit with fourth-order

polynomial functions

Figure 4.7 Time weighted average Rayleigh wave velocity fit with piecewise-linear

functions 
Figure 4.8 Semi-variogram and covariance function for time weighted average Rayleigh wave velocity not including data from after the hill........................................ 68 Figure 4.9 Semi-variogram and covariance function for time weighted average Rayleigh wave velocity with trend removed as a fourth-order polynomial function 69

Figure 4.10 Semi-variogram and covariance function for time weighted average Rayleigh wave velocity with trend removed as a piecewise-linear function 70

Figure 4.11 Semi-variogram and covariance function for time weighted average Rayleigh wave velocity with trend removed as a linear function (without data after hill)

Figure 4.12 Semi-variogram from Rayleigh wave data at 10m depth using all available data and being modeled with a spherical model (left) and a Gaussian model (right) 74

Figure 4.13 Overview of semi-variogram plots fitted with spherical models 75 Figure 4.14 Semi-variogram at 30 meters depth from Boore's estimate using velocity values at 10 meters depth; Empirical semi-variogram values (left) and semi-variogram with a Gaussian model (right) 78 


\section{Chapter 1: Introduction}

\subsection{Overview}

Lifelines do not typically have the benefit of being confined to a small area. Their long, linear nature implies that they will cover more ground, and this creates more opportunity for changing soil conditions beneath them. When designing these structures, it is important to know where to group similar or correlated material. The similarities and dissimilarities will govern where to test/sample the material and possibly where to change the design.

Europe and Russia both have codes that give guidance on these correlation distances. The European code (EN 1997-2: Eurocode 7: Geotechnical Design - Part 2: Ground Investigation and Testing, 2007) gives broad guidelines. In chapter 3, they recommend sampling based on investigations, the geology, and the complexity of the structure, but they do provide recommendations in Annex B.3 based on the type of structure. For linear structures such as "roads, railways, channels, pipelines, dikes, tunnels, [and] retaining walls," they recommend a sample spacing of between 20 and 200 meters. The Russian code (Engineering Surveys for Construction. Basic Provisions, 1996) has more specific guidelines for linear structures. They recommend the bandwidth zone (the transverse distance that points can be sampled between), the longitudinal distance between sample locations, and the required sample depth. A summary of the recommendations from the Russian code can be seen in Table 1.1. Note that this study is primarily interested in the longitudinal distance between samples, and this table shows 
the range to be between 100 and 1,000 meters (or 100 and 500 meters if power lines are ignored).

\begin{tabular}{|c|c|c|c|c|}
\hline Type of Linear Object & $\begin{array}{l}\text { Bandwidth of } \\
\text { Investigation } \\
\text { Zone }(\mathrm{m})\end{array}$ & $\begin{array}{l}\text { Longitudinal Distance } \\
\text { between Investigation } \\
\text { Points }(\mathrm{m})\end{array}$ & \multicolumn{2}{|c|}{ Sample Depth (m) } \\
\hline \multirow{2}{*}{$\begin{array}{l}\text { Railway } \\
\text { Highway }\end{array}$} & $200-500$ & $350-500$ & Up to 5 & \multirow{3}{*}{$\begin{array}{l}2 \text { m lower } \\
\text { than the } \\
\text { standard } \\
\text { depth of soil } \\
\text { freezing }\end{array}$} \\
\hline & $200-500$ & $350-500$ & Up to 3 & \\
\hline Main Pipeline & $100-500$ & $300-500$ & $\begin{array}{l}\text { At } 1-2 \mathrm{~m} \\
\text { below the } \\
\text { depth of the } \\
\text { pipeline }\end{array}$ & \\
\hline $\begin{array}{l}\text { Overpass for Terrestrial } \\
\text { Communication }\end{array}$ & 100 & $100-200$ & \multicolumn{2}{|c|}{$3-7$} \\
\hline \multicolumn{5}{|l|}{ Power Lines (kV): } \\
\hline Less than or equal to 35 & $100-300$ & $500-1000$ & \multicolumn{2}{|c|}{$3-5$} \\
\hline More than 35 & $100-300$ & $500-1000$ & \multicolumn{2}{|c|}{$7-10$} \\
\hline Cable Link & $50-100$ & $500-1000$ & \multirow{2}{*}{$\begin{array}{l}\text { At } 1-2 \mathrm{~m} \\
\text { below the } \\
\text { depth of the } \\
\text { pipeline }\end{array}$} & \multirow{2}{*}{$\begin{array}{l}\text { At } 1-2 \mathrm{~m} \\
\text { below the } \\
\text { standard } \\
\text { depth of soil } \\
\text { freezing }\end{array}$} \\
\hline $\begin{array}{l}\text { Water Supply, Sewerage, } \\
\text { Heating and Gas Pipelines }\end{array}$ & $100-200$ & $100-300$ & & \\
\hline $\begin{array}{l}\text { Underground Collectors - } \\
\text { Gutter and } \\
\text { Communication }\end{array}$ & $100-200$ & $100-200$ & \multicolumn{2}{|c|}{$\begin{array}{l}2 \mathrm{~m} \text { below the estimated depth } \\
\text { of the reservoir }\end{array}$} \\
\hline \multicolumn{5}{|c|}{$\begin{array}{l}\text { Notes } \\
\text { 1. Minimum distances should be used for complex geotechnical conditions and the maximum distances for } \\
\text { simple geotechnical conditions. }\end{array}$} \\
\hline \multicolumn{5}{|c|}{$\begin{array}{l}\text { 2. In areas with specific soils, the development of dangerous geological processes should reduce the } \\
\text { distance by three to five workings. } \\
\text { 3. If the corridor is supposed to trace the design of several linear features, the number and depth of } \\
\text { excavation is set based on the minimum distance and maximum depth for the corresponding linear objects. }\end{array}$} \\
\hline
\end{tabular}

Table 1.1 Typical design for bandwidth, sample spacing, and sample depth according to Russian code

America has yet to come up with recommendations for this sample spacing. This study's goal is to give a recommendation for this spacing. To do this, we collected velocity values in alluvial soil and found the correlation distance. The data was collected using the spatial autocorrelation method (SPAC) which gives Rayleigh wave and shear wave velocity values. These velocity values were then analyzed using spatial statistics to 
find the correlation distance. Since the correlation distance denotes when the soil becomes dissimilar, this distance can be assumed to be the maximum, allowable sample spacing.

\subsection{Organization of Thesis}

This thesis is written with the hope that any person with a moderate, technical background can understand the work. Background material is presented before delving into the results in order to provide the reader with an understanding and context of both surface-wave testing and geostatistical tools. The first part of chapter 2 reviews the concepts behind surface-wave testing, how to collect and analyze this kind of data, and some limitations behind the methods. The second part of chapter 2 reviews the concepts of geostatistics, its common functions and models, and some limitations associated with these tools. Chapter 3 goes through the hands-on aspect of surface-wave testing. It gives instructions on what equipment was used, how to use the equipment in the field, and stepby-step instructions on how to process the data. Then the results are presented in chapter 4 followed by the conclusion and recommendations for further research in chapter 5 .

\subsection{Geology of the Site}

An overview of the site's location (Cuesta College's campus) can be seen in Figure 1.1 (Google Maps, 2015). The surficial geology of this site and the surrounding area can be seen in Figure 1.2. It shows that the project lies on young, alluvial flood-plain deposits $\left(\mathrm{Q}_{\mathrm{ya}}\right)$ whose description is also shown in Figure 1.2. In the surrounding area, all of the Cretaceous to Jurassic units (KJ) shown in the figure are of the Franciscan complex. They consist of mélange $\left(\mathrm{KJ}_{\mathrm{fm}}\right)$, sandstone and shale $\left(\mathrm{KJ}_{\mathrm{fs}}\right)$, metavolcanic rocks $\left(\mathrm{KJ}_{\mathrm{fmv}}\right)$, and chert $\left(\mathrm{KJ}_{\mathrm{fch}}\right)$. Other nearby units consist of serpentinized, ultramafic rocks 
$\left(\mathrm{J}_{\mathrm{os}}\right)$, a volcanic intrusive complex $\left(\mathrm{T}_{\mathrm{d}}\right)$, landslide deposits $\left(\mathrm{Q}_{\mathrm{ls}}\right)$, alluvial flood-plain deposits $\left(\mathrm{Q}_{\mathrm{a}}\right)$, and felsite $\left(\mathrm{T}_{\mathrm{f}}\right)$.

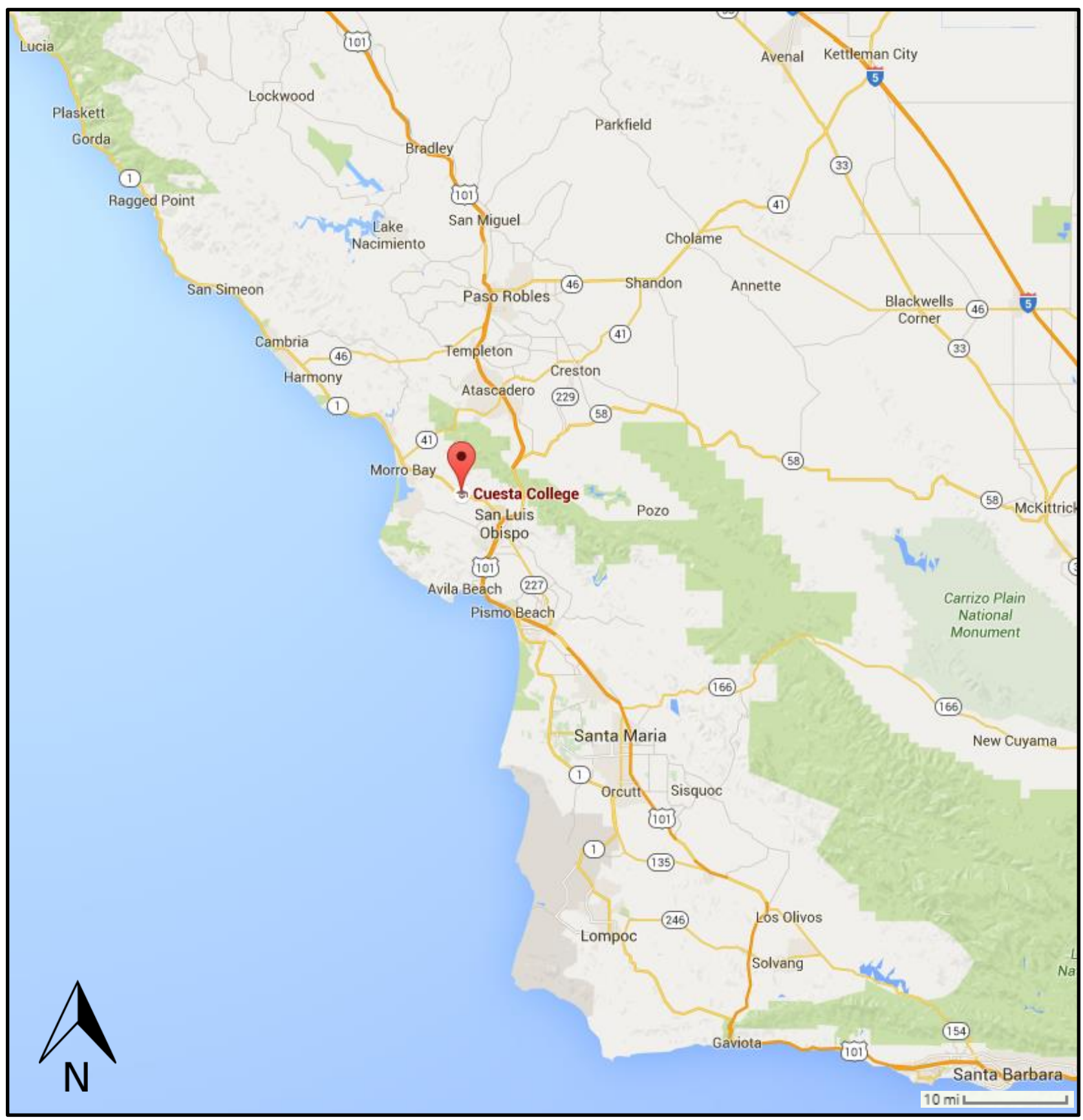

Figure 1.1 Vicinity map of the site 


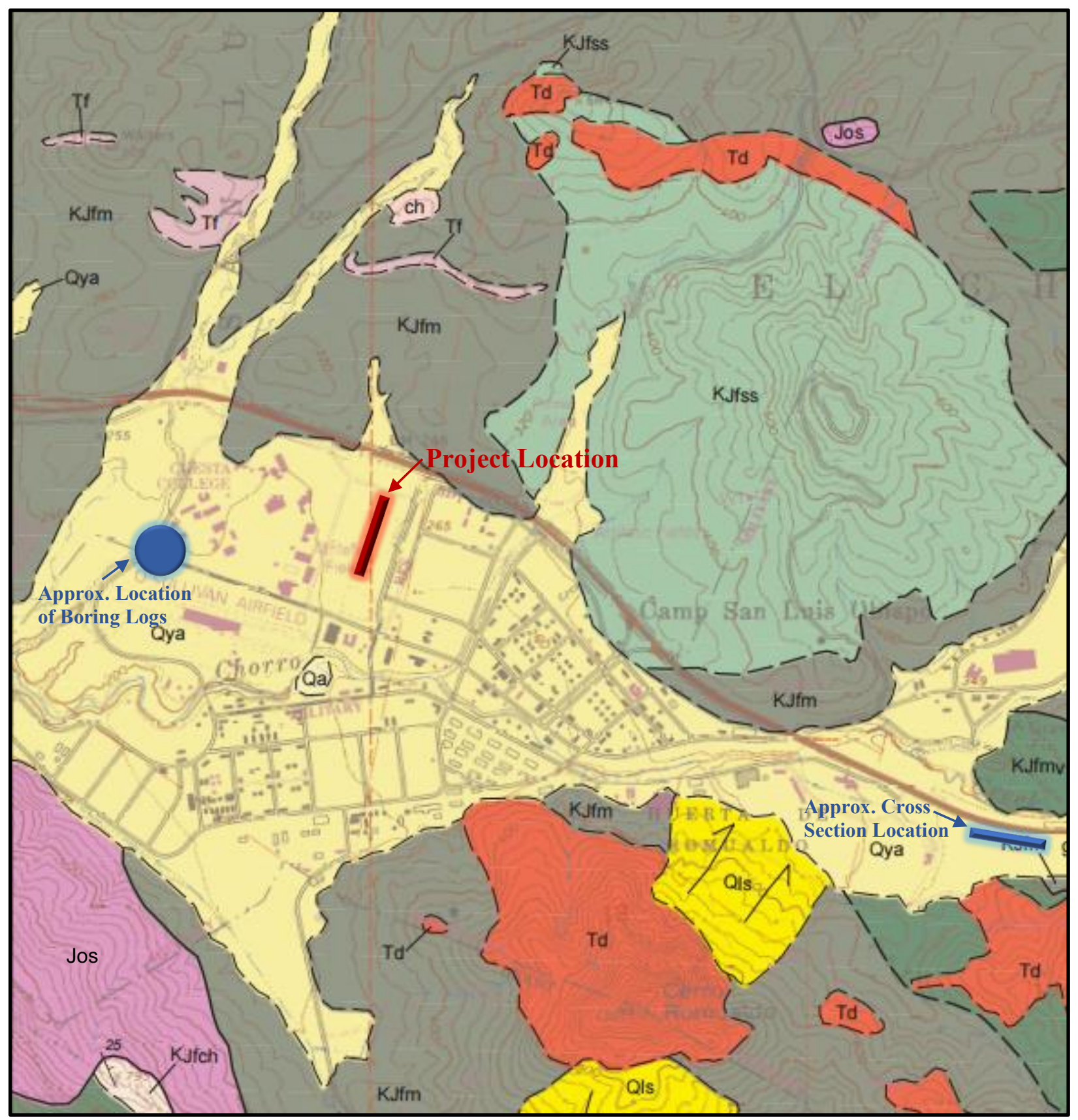

Young Surficial Deposits

Qya Young alluvial flood-plain deposits, undivided (Holocene to late Pleistocene) Unconsolidated sand, silt, and clay-bearing alluvium deposited on flood-plains and along valley floors. Locally divided by relative age $(2=$ youngest, $1=$ oldest):

Figure 1.2 Geologic map of the site and the surrounding area (California Conservation and California Geologic Survey, 2010) 
Chipping (1987) describes these units in detail. He writes that the alluvium is no thicker than 80 feet near the coast and thins to "probably" no more than 20 to 30 feet as one goes further inland. These sediments are relatively fine. He also explains that the Franciscan formation mélange is the most common rock form in the area which consists of a mixture of sandstone, chert, serpentine, basalt, greenstone, shale, and high-grade metamorphic rocks. The volcanic rocks are nearly all basaltic and can be found as blocks within the mélange or slab-like masses in the mélange that can stretch for miles.

Earth Systems Pacific also provided a nearby, geologic cross-section and eight boring logs from a project at Cuesta College. The cross section (from a report submitted in 2015) can be seen in Figure 1.3, and its approximate location can be seen in Figure 1.2. The boring logs are from a 2010 report and can be seen in the Appendix. Their approximate location can also be seen in Figure 1.2. The cross section shows alluvial material with a thickness ranging from approximately 30 to 60 feet (9 to 19 meters) being underlain by metavolcanic, serpentinite, and sandstone bedrock. The boring logs are all drilled to 11.5 feet (3.5 meters) and show sandy clay with some locations underlain by claystone (Franciscan formation). The three logs that show claystone give depths of 4 to 7 feet to this layer. The other five logs show sandy clay for the full 11.5 feet. 


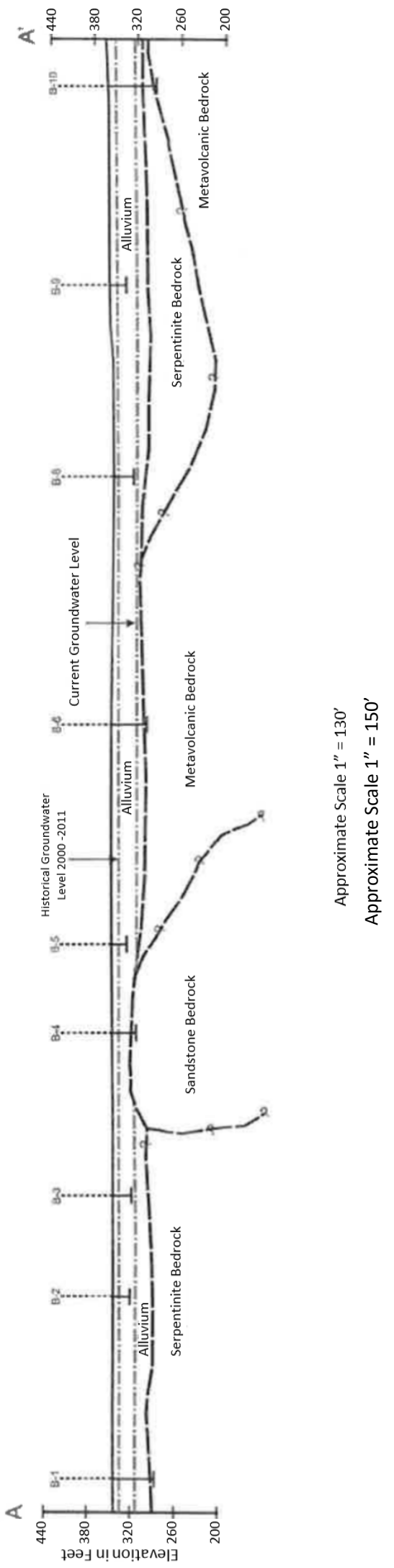

Figure 1.3 Cross section of nearby location (provided by Earth Systems Pacific) 


\section{Chapter 2: Literature Review}

The aim of this chapter is to give background knowledge of both the spatial autocorrelation (SPAC) method and of geostatistical tools so that a framework can be established before delving into the study's results. This overview is inherently broad due to the complexities of the subject matter, but references to other works are included if further depth is desired.

\subsection{Review of the Spatial Autocorrelation (SPAC) Method}

\subsubsection{Introduction to SPAC}

There are two types of seismic wave testing - invasive and noninvasive. Invasive testing acquires data from within the geologic material whereas noninvasive testing relies on data retrieved from the ground surface. Cross-hole, down-hole, sCPT, and suspension logging are examples of invasive testing. Noninvasive examples include refraction, reflection, and surface-wave testing. The benefit of invasive testing is that the uncertainty of the values (usually seismic velocity) is smaller since the data is recorded from inside the material, but it can be expensive to explore large areas. The opposite is true for noninvasive methods. At a cheaper cost, they can sample large amounts of material, but the values have greater uncertainty (Moss, 2008).

SPAC is a noninvasive test that was first introduced by Aki $(1957,1965)$. Aki took the idea of characterizing the earth's crust with earthquake tremors, and formed a method to characterize the earth's near-surface material by using microtremors. For SPAC, these microtremors consist of passive, ambient noise such as highway traffic, wind currents, and ocean waves. By recording these microtremors with a two-dimesional 
array, the direction of the wave propagation can be found and the speed of that wave can be used to estimate properties of the underlying geologic material.
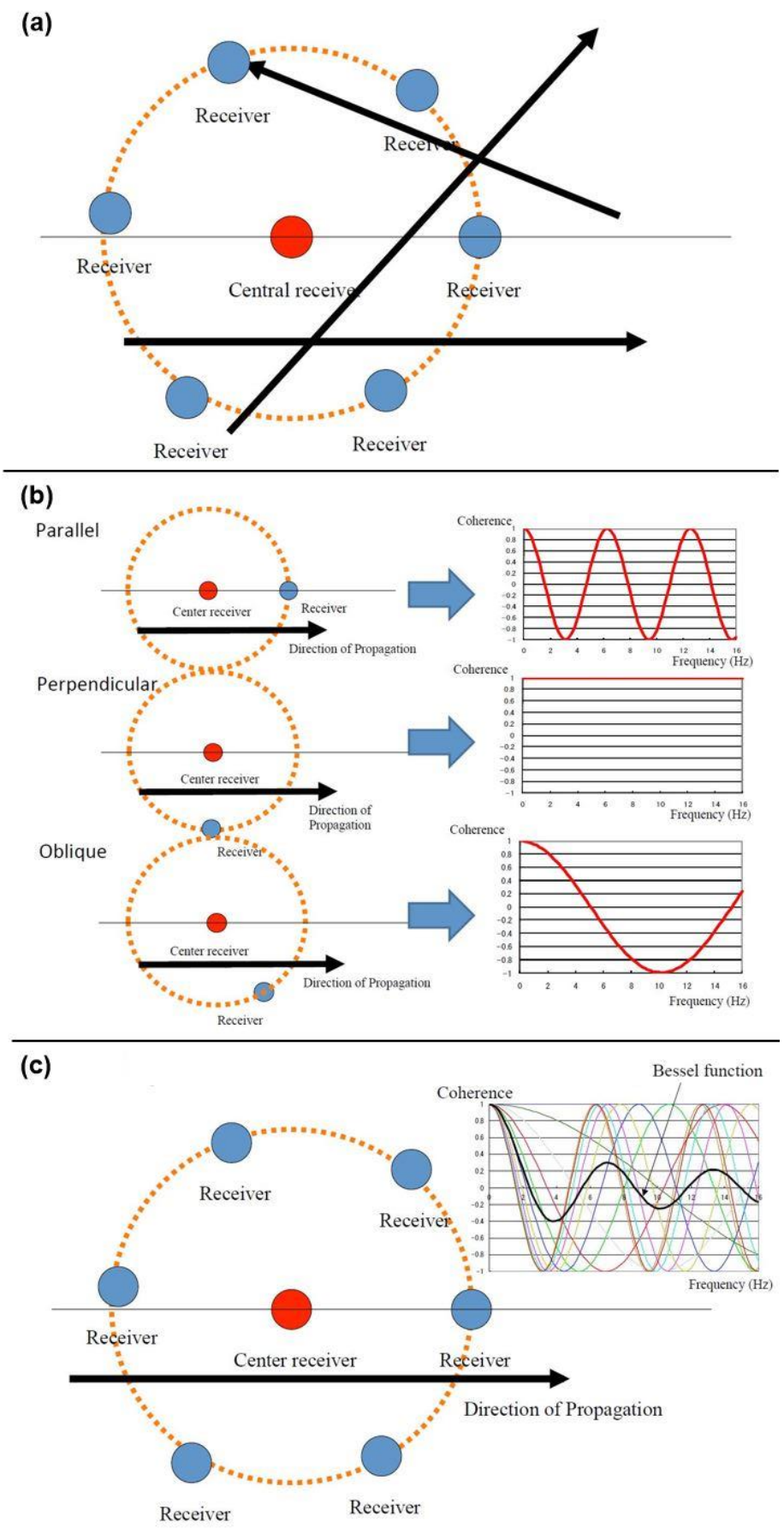

Figure 2.1 The derivation of spatial autocorrelation as presented by Kiochi Hayashi; (a) Waves propagating in different directions, (b) the coherence of the wave propagation between pairs of receivers, and (c) averaging the coherence between all receiver pairs to create a Bessel function 
Hayashi (2015) presented a visual for how SPAC identifies the direction of the wave propagation (Figure 2.1). The figure shows how these microtremors, in the form of surface waves, can propagate across an array (Figure 2.1a), and how the coherence of these waves can be plotted for each receiver paired with the central receiver (Figure 2.1b). This coherence between receiver pairs takes the form of a cosine function, and the cosine functions can be averaged to create a Bessel function (Aki, 1957) as shown in Figure 2.1c. This Bessel function is used to identify the velocity of the waves through the spatial correlations found between the array's receivers - hence the term spatial autocorrelation.

In mathematical terms, Malagnini et al. (1993) summarize Aki’s procedure succinctly. They show that, given a circular array with a receiver in the center, Aki defined the spatial correlation function as;

$$
\phi(r, \lambda)=\langle u(x, y, t) \cdot u(x+r \cos \lambda, y+r \sin \lambda, t)\rangle
$$

where $u(x, y, t)$ is the waveform/velocity observed at point $(x, y)$ at time $t, r$ is the radius of the circular array, $\lambda$ is the azimuth between each receiver and the central receiver, and $\langle\cdot\rangle$ indicates the azimuthal average. This azimuthal average (the average of the functions between each receiver and the central receiver) can be shown to equal;

$$
\phi(r)=\frac{1}{\pi} \int_{0}^{\pi} \phi(r, \lambda) d \lambda
$$

Aki (1957) showed that this function can be related to the power spectrum, $\Phi(\omega)$, by the zeroth-order Hankel transform to give the equation;

$$
\phi(r)=\frac{1}{\pi} \int_{0}^{\infty} \Phi(\omega) J_{o}\left(\frac{\omega r}{c(\omega)}\right) d \omega
$$


where $\omega$ is the angular frequency, $J_{o}\left(\frac{\omega r}{c(\omega)}\right)$ is the zeroth-order Bessel function of the first kind, and $c(\omega)$ is the phase velocity of the wave which varies with frequency. Note that dispersive waves are characterized by two types of velocity - phase velocity and group velocity (Rayleigh, 1877). The phase velocity is the speed of a single phase of the waveform, whereas the group velocity is the velocity of a packet of "group" waves. Think of the waves of a ripple in a pond; the waves move up and down at a certain speed but the packet of waves disperse at another speed. Phase velocities are the ones used in SPAC; group velocities are rarely used for near-surface applications (Foti et al., 2014). Aki goes on to show that the phase velocity can be found if the recordings are bandpass filtered over a frequency range centered on the fundamental frequency, $\omega_{o}$, and the spatial correlation function is normalized to the power spectrum which gives the equation;

$$
\phi\left(r, \omega_{o}\right)=J_{o}\left(\frac{\omega_{o} r}{c\left(\omega_{o}\right)}\right)
$$

Later, Bracewell (1978) used similar logic and formulated equations to find a wave's phase velocity from an array without a central receiver.

\subsubsection{Acquisition Equipment}

The receivers used in this study were $4.5 \mathrm{~Hz}$ geophones which are a form of velocimeter (i.e. electrodynamic velocity transducer). They have the ability to record very small displacements that, in this study, come from vibrations in the soil. They are typically of the moving coil type which houses a coil that sits in a magnetic field. This coil is suspended by a spring(s) which encompasses the magnet that produces the magnetic field. The magnet is permanently fastened to the casing so that, when the 
geophone moves, the relative movement of the coil creates a small voltage that is proportional to the relative velocity of the geophone (Foti et al., 2014). A visual of the type of geophone used in this study and a cross-section of a typical moving coil type geophone can be seen in Figure 2.2.

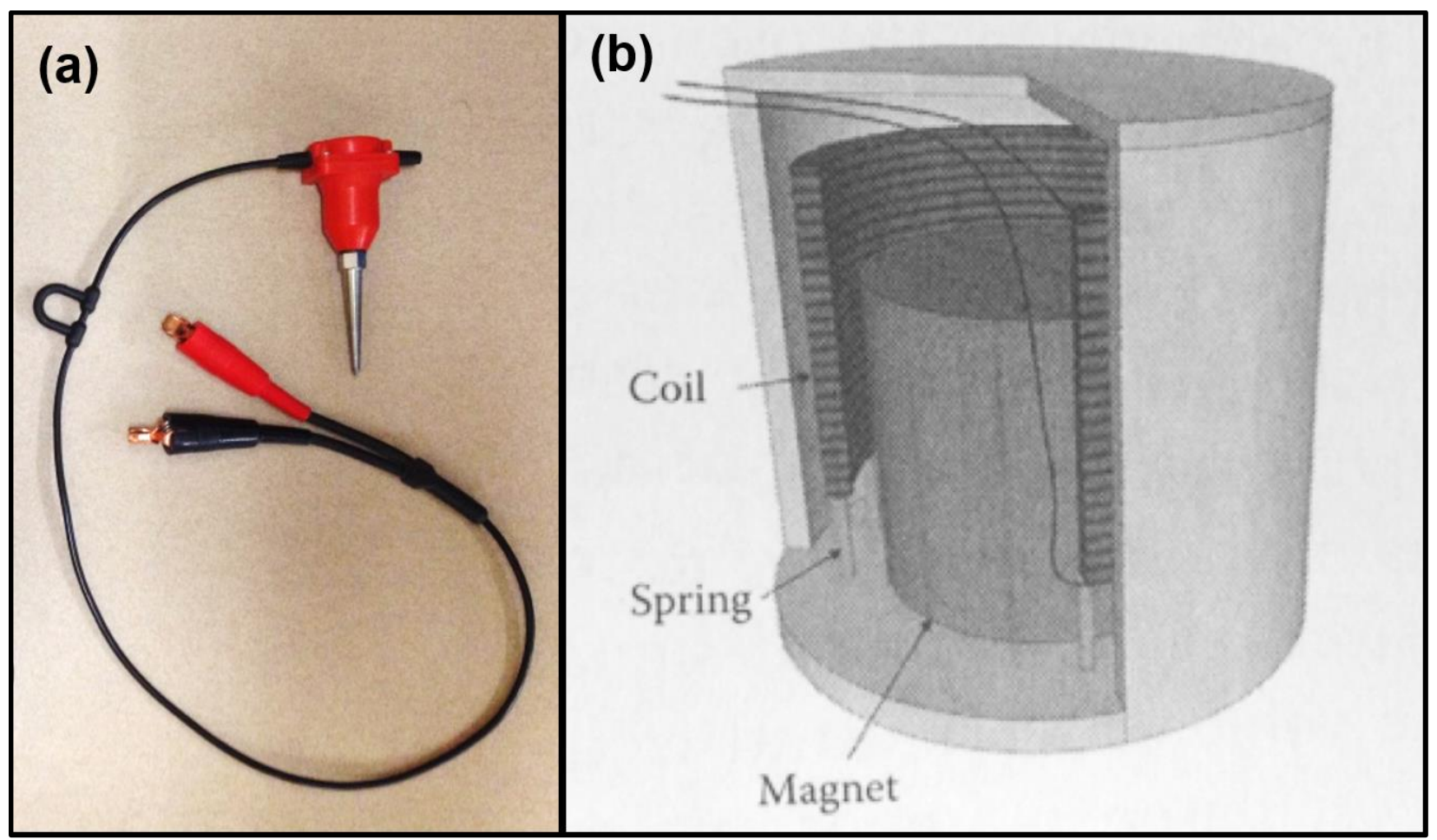

Figure 2.2 Geophone visual; (a) $4.5 \mathrm{~Hz}$ geophone used in this study and (b) cross-section of a typical moving coil type geophone (Foti et al., 2014, p. 193)

The geophone sends the analog information to the data acquisition (DAQ) system which digitizes the signal. As well as conditioning the data, the DAQ system also directs the sampling rate which needs to be high enough to avoid aliasing. Aliasing can create distortions and artifacts in the data that can lead to erroneous analysis. However, the Nyquist-Shannon theorem says that no information will be lost by regular sampling if the sampling frequency is two times greater than the highest frequency of the sampled signal (Telford and Geldart, 1990). For example, this study used a sampling frequency of 2 
milliseconds which corresponds to $500 \mathrm{~Hz}$, so no information will be lost for wave frequencies that are less than or equal to $250 \mathrm{~Hz}$.

\subsubsection{Surface Wave Measurement and Analysis}

Rayleigh waves are the type of surface wave analyzed in this study. Other studies have used Love waves, but their horizontal particle motion requires horizontally-oriented receivers and Love waves will not form if a low-velocity layer exists below a highervelocity layer (Hudson, 1980). In heterogeneous material, both types of waves are geometrically dispersive, meaning that their velocity depends on the wave's frequency (Aki and Richards, 2002). Different frequencies will sample different depths of the geologic material. Higher-frequency waves sample shallower material and lowerfrequency waves sample deeper material. This can be more easily explained in terms of wavelength (which is inversely proportional to frequency); waves with larger wavelengths will reach deeper into the surface and will sample more material than waves that have shorter wavelengths. An illustration of this can be seen in Figure 2.3.

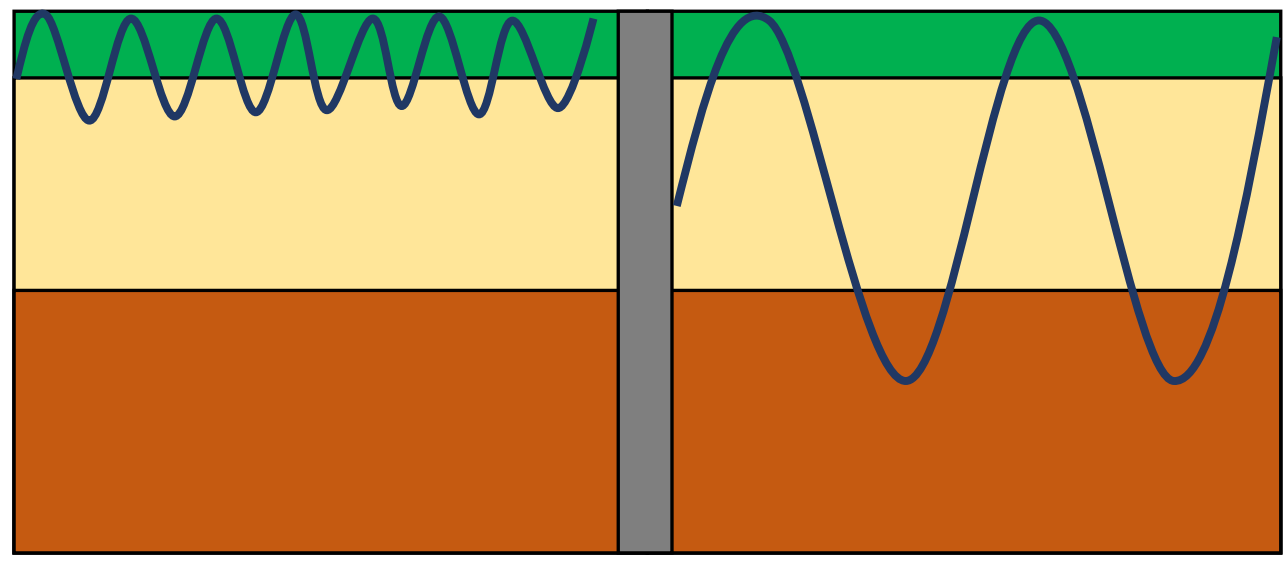

Figure 2.3 Illustration showing how shorter wavelengths (left) sample shallower material and longer wavelengths (right) sample deeper material 
Waves with large wavelengths/low frequencies tend to have higher velocity values since deeper material tends to be stiffer than shallower material. For passive waves, the wave source is not controlled, but it is assumed that a broad frequency range will come from the ambient noise. Waves with frequency ranges below $1 \mathrm{~Hz}$ are mainly generated from global, geophysical events (ocean waves in particular), and waves with frequency ranges above $1 \mathrm{~Hz}$ are mainly generated by human events such as traffic and industrial activities (Foti et al., 2014). As a rule of thumb, the particle motion from a wave is confined to one wavelength from the free surface (Achenbach, 1973). So wavelengths that are smaller than the thickness of the first layer will only sample material from that first layer, but larger wavelengths will sample multiple layers and the velocity of these large-wavelength waves will be governed by some combination of the properties within each layer.

The array diameter is one of the main factors that controls what size waves will be recorded and, therefore, how deep the array will sample. There are different recommendations for how large to make an array if a certain wavelength is desired. Tokimatsu (1995) recommends an array diameter that is one-third of the desired wavelength and studies like Asten and Henstridge (1984) use an array diameter equal to the desired wavelength. But even when a wavelength is recorded, the velocity values are only accurate to a certain depth. Through analysis of variability, Rix and Leipski (1991) found that velocities could be accurately estimated at a depth equal to half of the wavelength. Other studies have recommended different fractions of the wavelength (e.g. Joyner et al., 1981), but Rix and Leipski's factor of 0.5 is the one used in this study. 
Once the waves are recorded, analysis of these waves is done in the frequency domain since their velocity is frequency dependent. This analysis is usually carried out by generating a dispersion curve (an example of which can be seen in Figure 2.4).

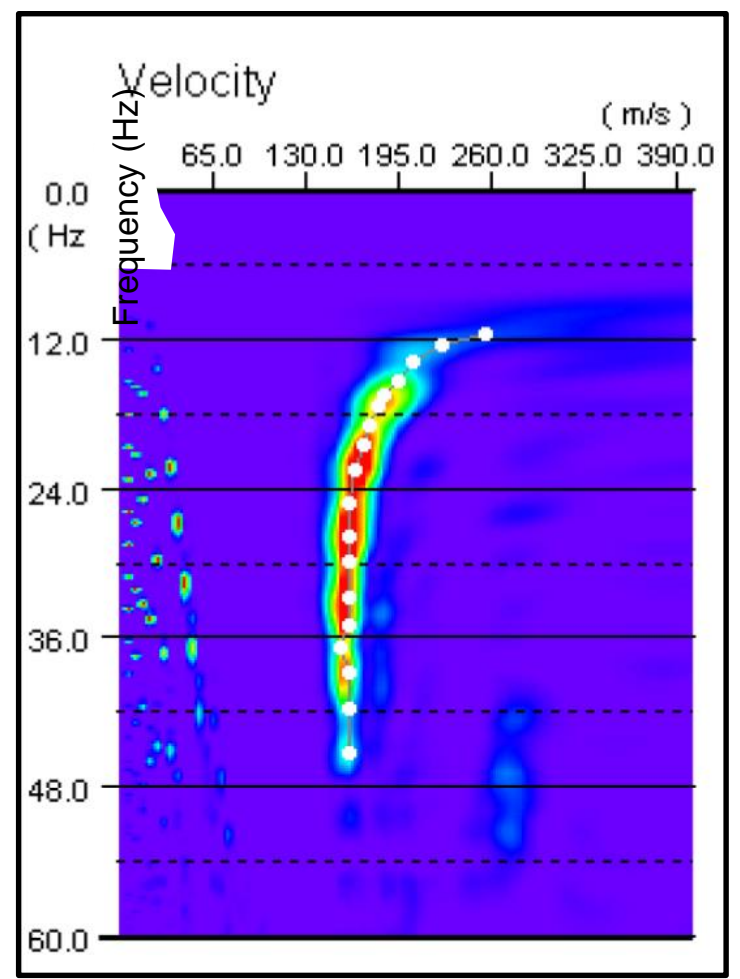

Figure 2.4 Example dispersion curve from the Geogiga Surface Plus (2012) manual The dispersion curve shows the relationship between the phase velocities and their corresponding frequencies. This curve is generally the goal of surface wave testing because an inversion process can be used to find the shear wave velocity profile, and shear wave velocity is commonly used to predict material properties or behavior (e.g. Andrus and Stokoe, 1996, Tezcan et al., 2006, and Thitimakorn, 2013). The dispersion curve resembles a decreasing exponential curve for sites where the geologic material gets progressively stiffer with depth. This geologic behavior is common, even in homogenous soil, because the weight of the overlying material tends to compress the material below which creates stiffer units and higher velocity values (Santamarina et al., 2001). This 
behavior is referred to as being normally dispersive. If the site does not follow normally dispersive behavior, the dispersion curve will depart from the likeness to an exponential curve.

Not only will the dispersion curve change with different material layering, it will also change depending on what mode of propagation is recorded. Figure 2.5 shows an example of how the dispersion curve can change depending on what mode is recorded.

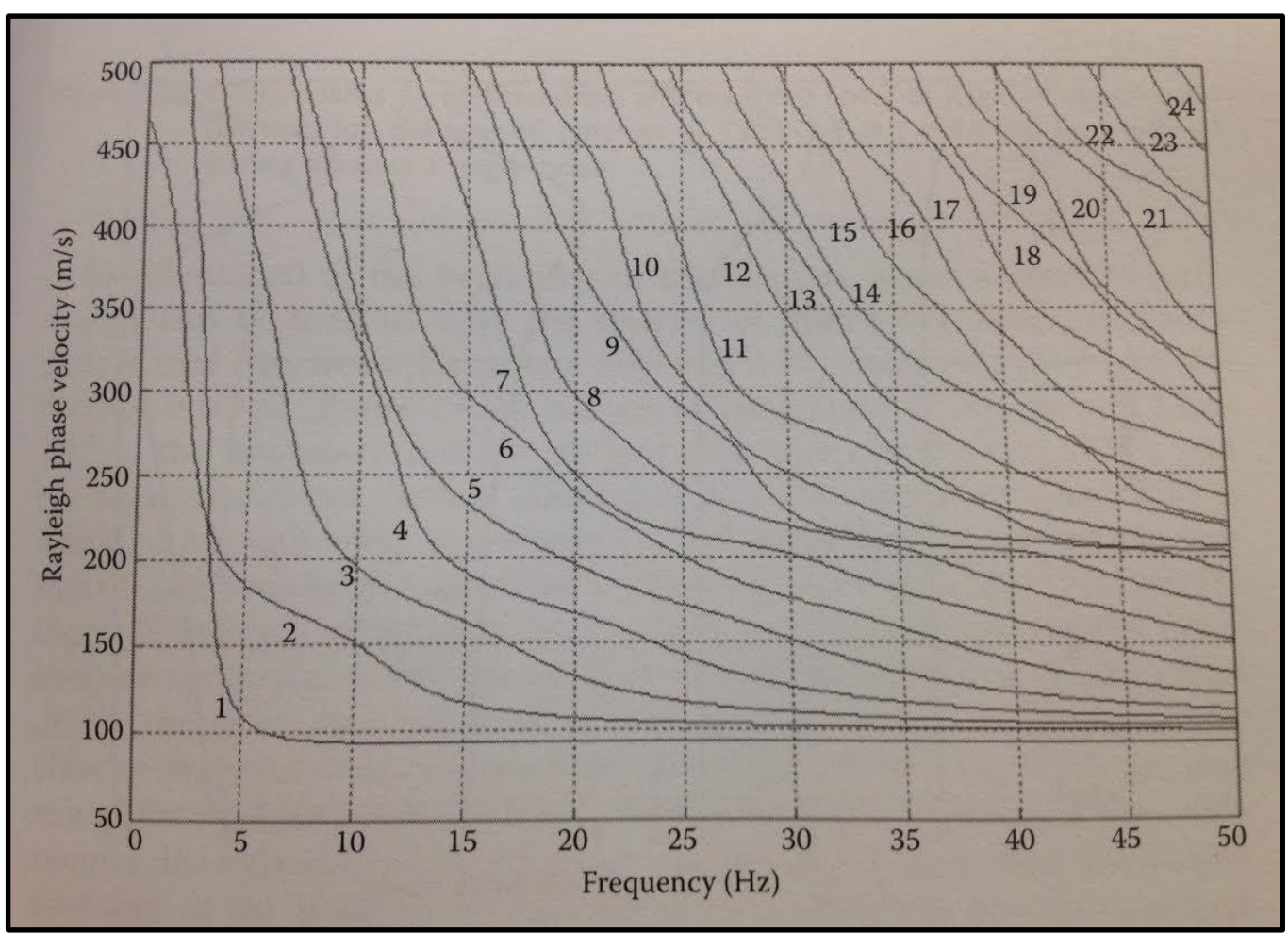

Figure 2.5 An example of how dispersion curves change with differing modes (Foti et al., 2014, p. 75); Each curve represents a single mode of propagation

Multiple modes will sometimes contribute to a dispersion curve, but current methods have difficulty identifying anything but the first mode of propagation (Foti et al., 2014). For normally dispersive sites, the propagation is dominated by the fundamental mode, so the dispersion curve can be assumed to be from the fundamental mode (Lai, 2005). There 
can be more contribution from higher modes if a site is inversely dispersive (if highvelocity layers overly low-velocity layers), as seen in Figure 2.6.
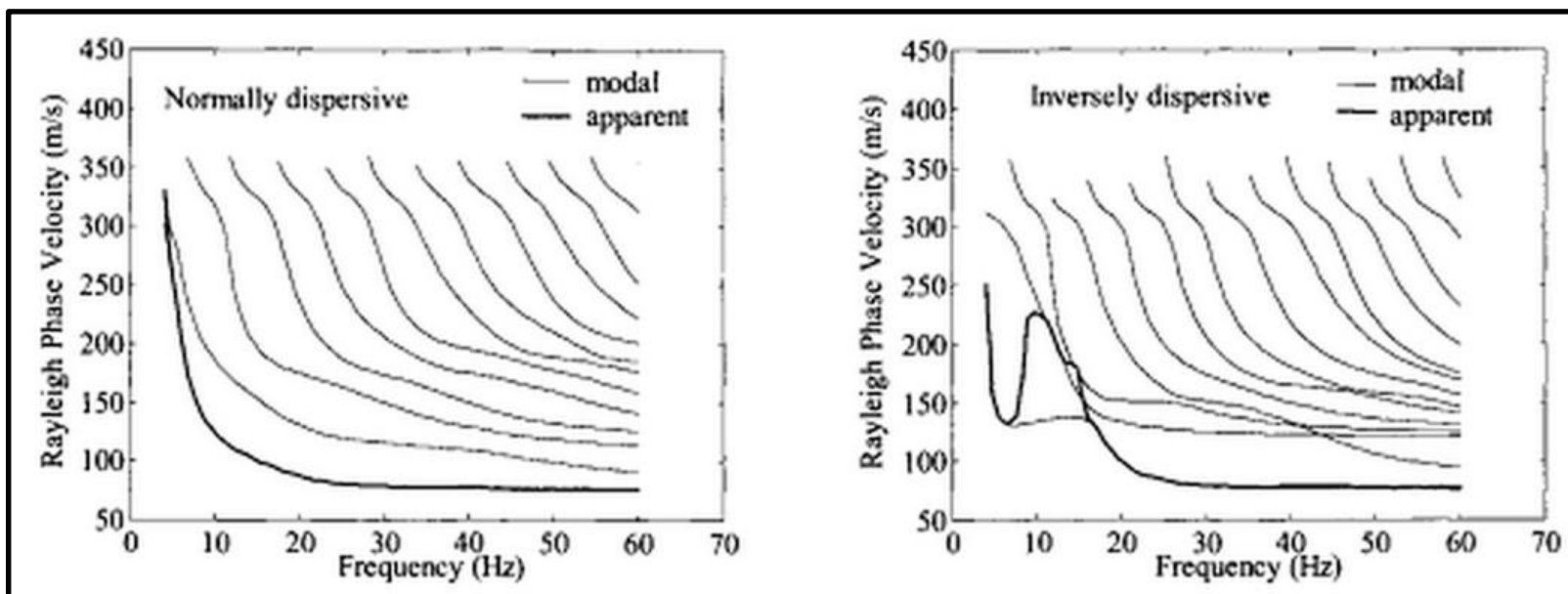

Figure 2.6 Modal and apparent Rayleigh dispersion curves for normally dispersive (left) and inversely dispersive (right) material (Lai, 2005, p. 116)

\subsubsection{Inversion}

After the dispersion curve has been identified, an inversion process can be used to create a shear wave velocity profile. This inversion process is typically carried out by a computer program (this study used the software Geogiga Surface Plus, 2012). The goal of the process is to create a soil profile that has a theoretical dispersion curve that matches the dispersion curve observed in the field. It does this by assuming a profile and iterating until the theoretical curve aligns with the observed curve. The most common inversion techniques use least squared regression to fit the theoretical dispersion curve to the observed curve (Foti et al., 2014). The residuals from this technique give a misfit error which tells how well the curves match. Through continued iteration, the goal of the process is to minimize the misfit error. The software used to analyze the data in this study (Geogiga Seismic Pro, 2012) uses a genetic algorithm to perform the inversion. 
Foti et al. (2014) describe how these inversion processes function. Ordinarily, the inversion of surface wave data is carried out by a local search procedure as opposed to a global search procedure. Most of these local search procedures use calculus-based methods that linearize a non-linear functional (the dispersion curve, in this case) at each iteration until a stationary point is reached. The dispersion curve needs to be sufficiently smooth for this to happen - smooth enough for its Frechet derivatives to exist. The Frechet derivative is outside the scope of this study, but can be seen as a generalization of the gradient to arbitrary vector spaces (Stover and Weisstein). Along with a sufficiently smooth curve, the solution will only converge to one that is similar to the field conditions if the initial estimate is sufficiently close to the solution (Virieux and Operto, 2009). In other words, the velocity model that is initially assumed needs to be similar to the actual model in order to have reliable results. This makes a priori information a valuable asset. Nearby borings, layer thicknesses, and approximate velocities are examples of information that would help constrain the inversion process. Most programs can build an initial model automatically, but this model is built assuming that the profile is normally dispersive (Foti et al., 2014). If the profile is not expected to be normally dispersive, caution should be used when automatically building an initial model as the results will favor a normally dispersive profile.

\subsubsection{Limitations}

During data acquisition, it is assumed that the waves being recorded are surface waves which are coming from an isotropic wave field and entering the array as a plane. If the waves are recorded too close to the source then body waves could be influencing the data, although this usually poses more problems for active sources than for passive 
sources. At 1 to 2 wavelengths from the source, the contribution of body waves becomes negligible and the wave field is dominated by Rayleigh waves (Lamb, 1904). For passive testing with a two-dimensional array, these waves need to be coming from an isotropic wave field (Horike, 1985). This isotropic wave field is required because the SPAC method assumes that random waves are entering the array and are propagating in all directions with equal probability. This ideal situation may be reasonably assumed if there are multiple, spatially distributed sources (Foti et al., 2014). The assumption that the waves are entering the array as a plane is also due to an assumption in the analysis. Rectangular coordinates are used when analyzing surface wave data, but polar coordinates would be needed if the array was too close to the source (Foti et al., 2014). As a rule of thumb, the closest geophone should be 1.5 to 2 wavelengths away from the source in order to avoid this (Foti et al., 2014). Along with these, noise in the recording can also cause large uncertainties when creating the dispersion curve (O’Neill, 2003).

Once the waves are recorded, there are limitations in the inversion processing. The main one being that the inversion process is not unique; a dispersion curve can correspond to many different shear wave velocity profiles (Lai et al., 2005). The only aid to this problem is the use of a priori information. Once the profile is created, the resolution of the model is the other key issue. The more layers a model has, the more uncertainty will be associated with each layer (Parker, 1977). Foti et al. (2014) shows this in Figure 2.7 where the standard deviations for a five-layer model are much smaller than those for a ten-layer model when all other variables are held constant. 


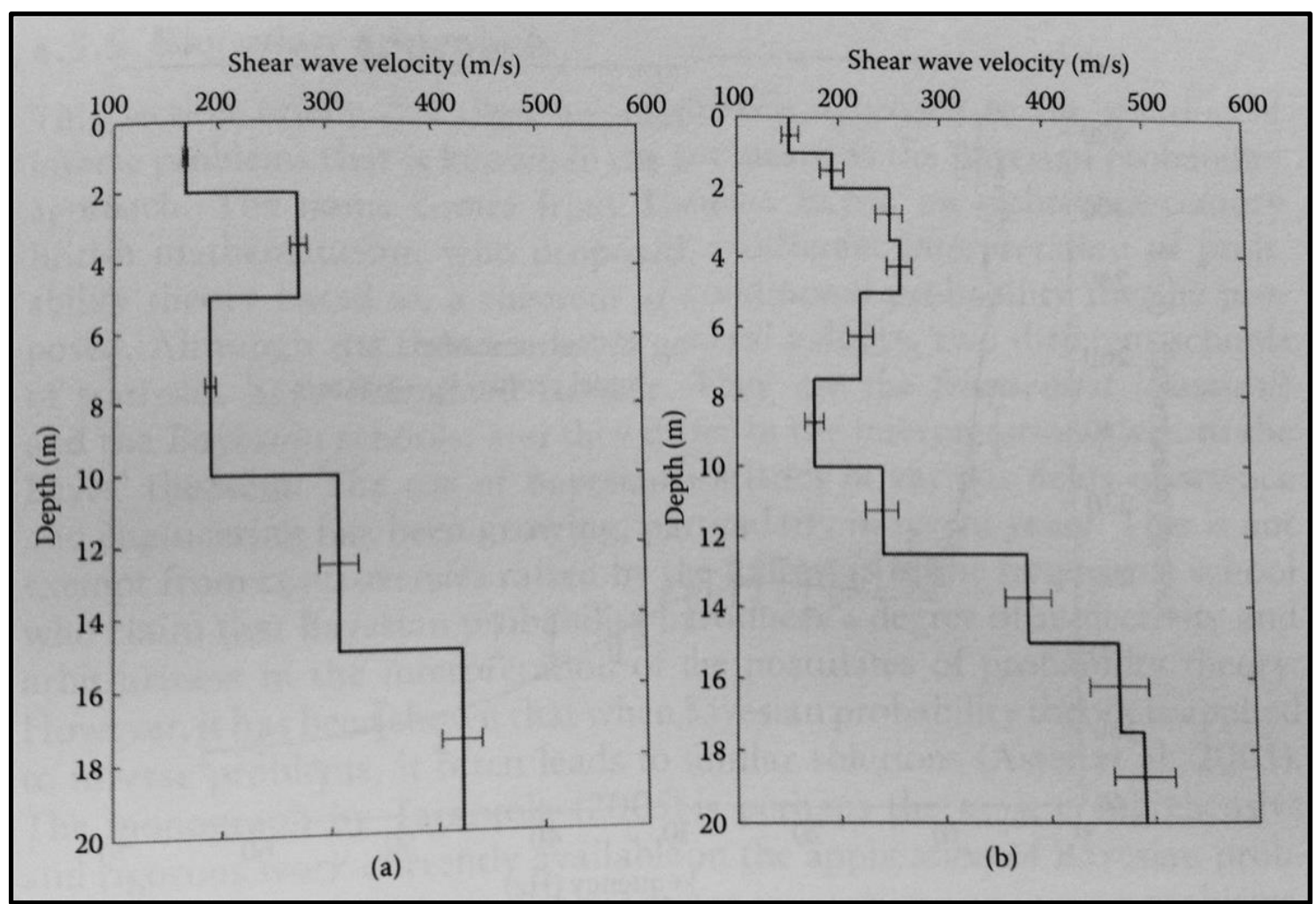

Figure 2.7 Standard deviations for Vs models that only differ by number of layers; (a) five-layer model and (b) ten-layer model (Foti et al., 2014, p. 347)

Notice that the uncertainties at depth, even for the five-layer model, are greater than those at shallower depths. This is common, as shown in research like Tuomi and Hiltenum (1996), Marosi and Hiltunen (2004), and Lai et al (2005). This is because there are, generally, a large amount of rays (wave propagations) that define the shallow layers, but few rays are recorded that define the layers at depth (Foti et al., 2014). An additional factor that causes this loss of resolution is random error which is introduced during the acquisition of the data. Lai et al. (2005) have shown that random errors are present in the dispersion curve values and that these errors cause loss of resolution. They also found that these errors increase with decreasing frequency. All of this makes deep, thin layers very hard to distinguish. 


\subsection{Review of Geostatistical Methods}

Most studies use geostatistical tools to find the variability and the correlation of some material parameter in order to estimate the value of a parameter at unsampled locations. The estimation is most commonly done via kriging. This study uses the tools to find the correlation range - not to estimate unsampled locations. Since the objective of this study is to find a range, estimation is unnecessary (and redundant due to the proximity of the surveys). Because of this, the following review of geostatistics is tailored towards variance rather than estimation.

\subsubsection{Introduction to Geostatistics}

The goal of geostatistics is to see how spatial data varies at certain distances. This is done by comparing each data point to the others and seeing how similar these data pairs are given their spacing. The comparison can be done graphically; for data separated by a certain lag distance, $h$, the data pairs can be plotted on a scatterplot. This is called an h-scatterplot, where $\mathrm{h}$ is defined as the lag/distance that separates the data pairs. Notice that the values will cluster around a $45^{\circ}$ line if they are similar. On average, most geologic data sets are similar at close lag distances (high values are close to high values and vice versa) and dissimilar at far lag distances (Isaaks and Srivastava, 1989).

Figure 2.8 shows an example of an h-scatterplot for five data points using a lag distance of 5 units where $h$ represents the spacing between the data pairs, $V(x)$ represents the first point of the data pair, and $V(x+h)$ represents the second point of the data pair. Notice how data pairs that have similar values stay close to the diagonal whereas dissimilar pairs plot further from this line. H-scatterplots can be created for additional lag distances to see the similarities or dissimilarities at other separations, and different ways 
of quantifying the clustering around the $45^{\circ}$ line will tell how spatially continuous the data is. One way of achieving this is to find the correlation coefficient for each hscatterplot and to plot this correlation value for each lag distance. An example of the correlation coefficient with lag distance can be seen in Figure 2.9. The plot ranges from 1.0 being perfectly correlated to 0 being uncorrelated, and it shows that data pairs at close separation are similar but those at longer separations are minimally correlated.

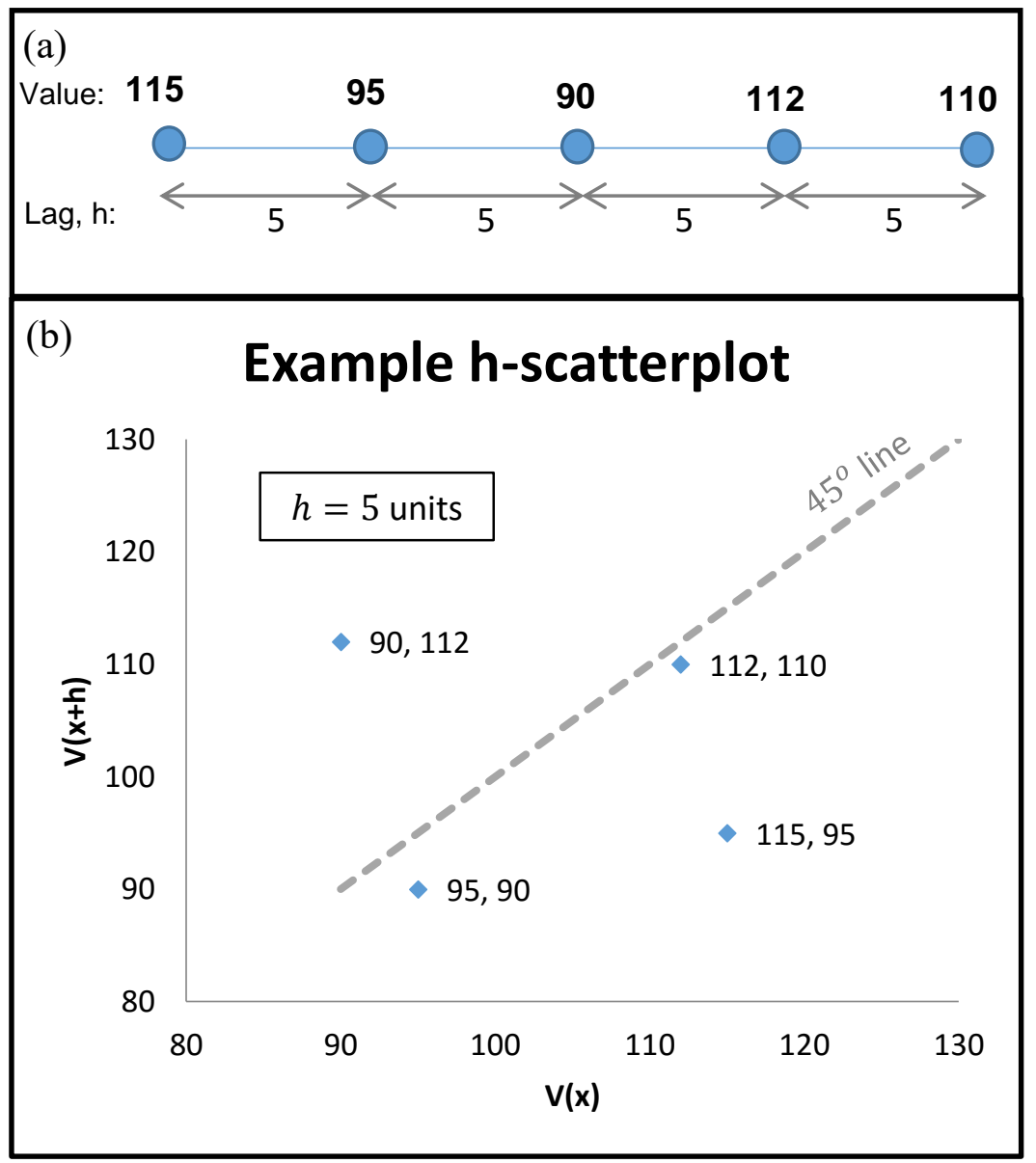

Figure 2.8 Example h-scatterplot; (a) values along a line with 5 unit spacing, and (b) the corresponding h-scatterplot 


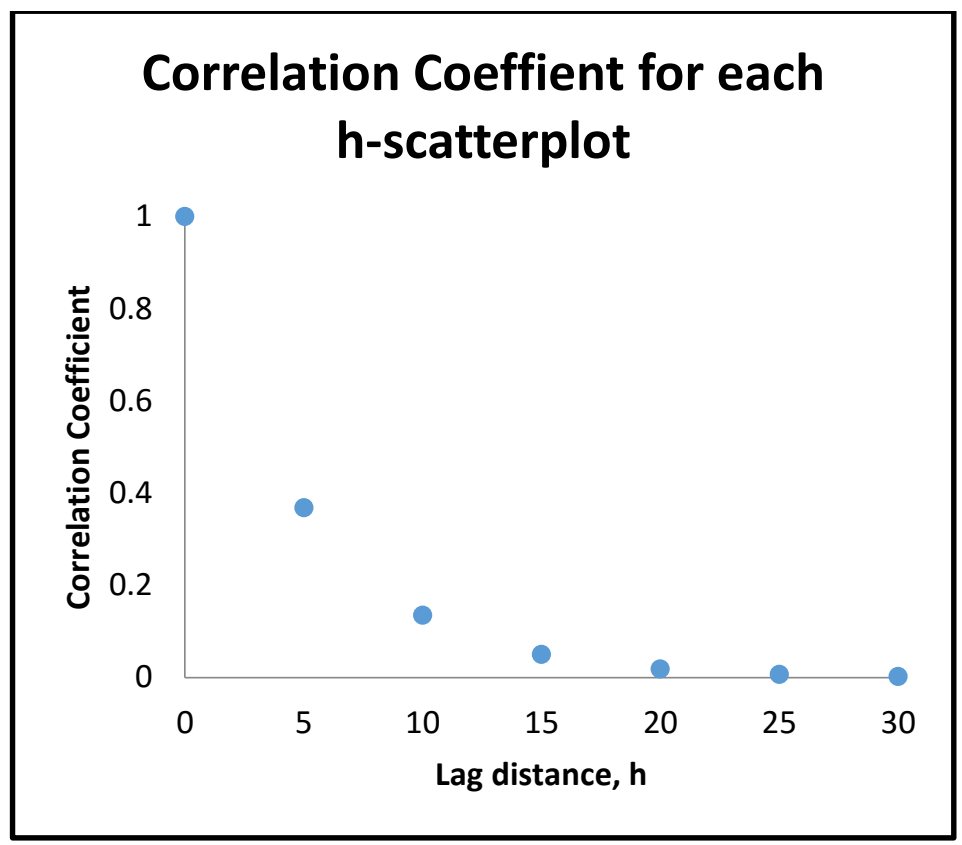

Figure 2.9 Example of how the correlation coefficient varies with lag distance

Other ways of quantifying the h-scatterplot is to find the covariance of the plot.

The definition of covariance is similar to that of correlation and, because of this, these plots share the same shape. Another way to model the h-scatterplot data is to find the moment of inertia around the $45^{\circ}$ line as done by Matheron (1965). Since the moment of inertia measures the "fatness" of the cloud of data, this value should increase with increasing lag. This is because nearby data (data with small lag distances) are expected to be similar and as the lag distance gets larger the data pairs are expected to become more and more dissimilar. On the h-scatterplots, this will show points close to the $45^{\circ}$ line at small lag distance and points further from the $45^{\circ}$ line (increasing "fatness") at larger lag distances. This expansion of points will be reflected by an increase in the moment of inertia value. An example of this can be seen in Figure 2.10. 


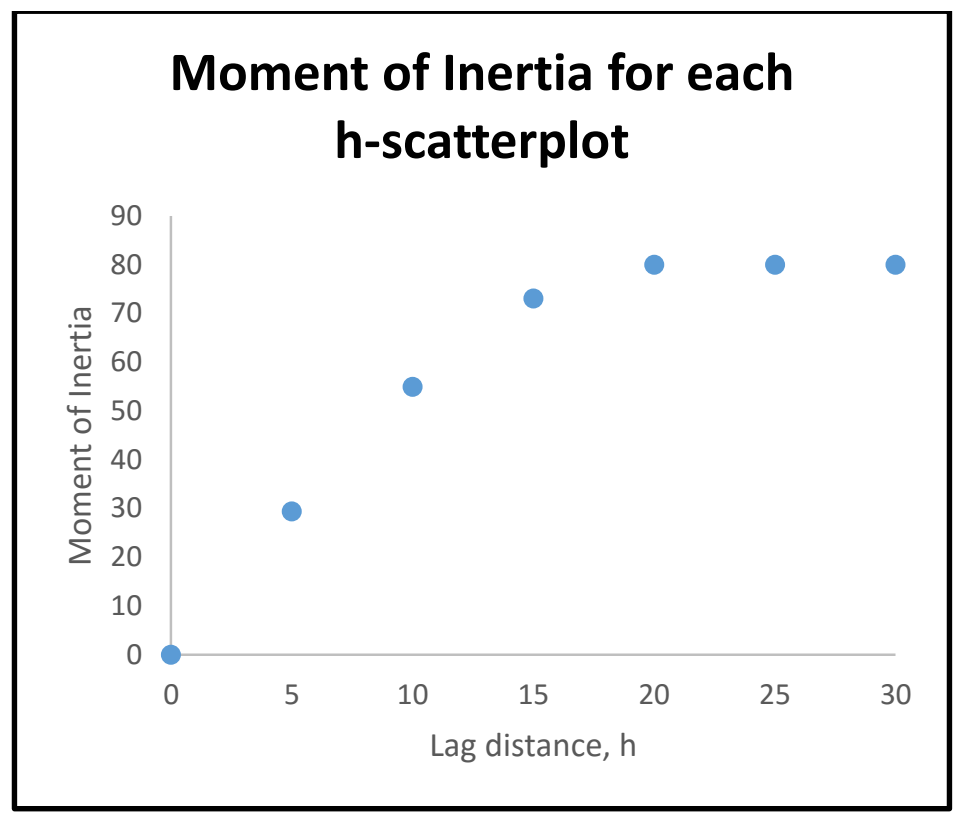

Figure 2.10 Example of how the moment of inertia varies with lag distance The moment of inertia about the diagonal of the h-scatterplots is defined as $\frac{1}{2 n} \sum\left(x_{i}-y_{i}\right)^{2}$, where $n$ is the number of data pairs, $x_{i}$ is the value $V(x)$, and $y_{i}$ is the value at a certain lag distance $V(x+h)$. The factor of $1 / 2$ is present because we are interested in the perpendicular distance away from the diagonal line.

The relationship between either the moment of inertia, the covariance, or the correlation coefficient with the lag distance are three common descriptors in geostatistics. The relationships are referred to as the semi-variogram, the covariance function, and the correlogram, respectively.

\subsubsection{Spatial Continuity Functions}

To save time, it is common to skip plotting each h-scatterplot and instead to graph the semi-variogram, the covariance function, or the correlogram directly. Equations have been formulated to do just this. The semi-variogram can be plotted by using the equation; 


$$
\gamma(\boldsymbol{h})=\frac{1}{2 N(\boldsymbol{h})} \sum_{(i, j) \mid h_{i j}=h}\left(v_{i}-v_{j}\right)^{2}
$$

where the data values are $v_{1}, \ldots, v_{n}$ and the summation is over the $N(\boldsymbol{h})$ data pairs whose locations are separated by $h$. Notice that $h$ is bolded in the equation because it represents multiple values instead of being a constant. The semi-variogram represents half of the average squared difference between data pairs, and can be shown to equal the variance of the values being estimated (Isaaks and Srivastava, 1989, p. 221-222).

In similar notation, the covariance function can be calculated using;

$$
C(\boldsymbol{h})=\frac{1}{N(\boldsymbol{h})} \sum_{(i, j) \mid h_{i j}=h} v_{i} \cdot v_{j}-m_{-h} \cdot m_{+h}
$$

where $m_{-h}$ and $m_{+h}$ are the mean values of all the data points whose locations are $-h$ and $+h$ away from some other data location, respectively. In other words, for each data pair, $-h$ denotes the first point and the $+h$ denotes the second point. These mean values are defined as;

$$
\begin{aligned}
& m_{-h}=\frac{1}{N(\boldsymbol{h})} \sum_{i \mid h_{i j}=h} v_{i} \\
& m_{+h}=\frac{1}{N(\boldsymbol{h})} \sum_{j \mid h_{i j}=h} v_{j}
\end{aligned}
$$

and they typically do not equal each other in practice (Isaaks and Srivastava, 1989).

The correlogram is the same as the covariance function, but is scaled by the standard deviations of the data pairs. It is defined as;

$$
\rho(\boldsymbol{h})=\frac{C(\boldsymbol{h})}{\sigma_{-h} \cdot \sigma_{+h}}
$$


where $\sigma_{-h}$ and $\sigma_{+h}$ are the standard deviations of all the data values whose locations are $-h$ and $+h$ away from some other data location, respectively. Their equations are;

$$
\begin{aligned}
\sigma_{-h}^{2} & =\frac{1}{N(\boldsymbol{h})} \sum_{i \mid h_{i j}=h} v_{i}^{2}-m_{-h}^{2} \\
\sigma_{+h}^{2} & =\frac{1}{N(\boldsymbol{h})} \sum_{j \mid h_{i j}=h} v_{j}^{2}-m_{+h}^{2} .
\end{aligned}
$$

The shape of a typical semi-variogram and covariance function is shown in Figure 2.11. Notice that they are the same except for being flipped horizontally (this happens if the data has a constant mean value). The semi-variogram ranges from 0 to its plateau whereas the covariance function starts at the semi-variogram's plateau and decreases to 0 . The correlogram matches the shape of the covariance function, but is scaled so that the maximum value is 1 .

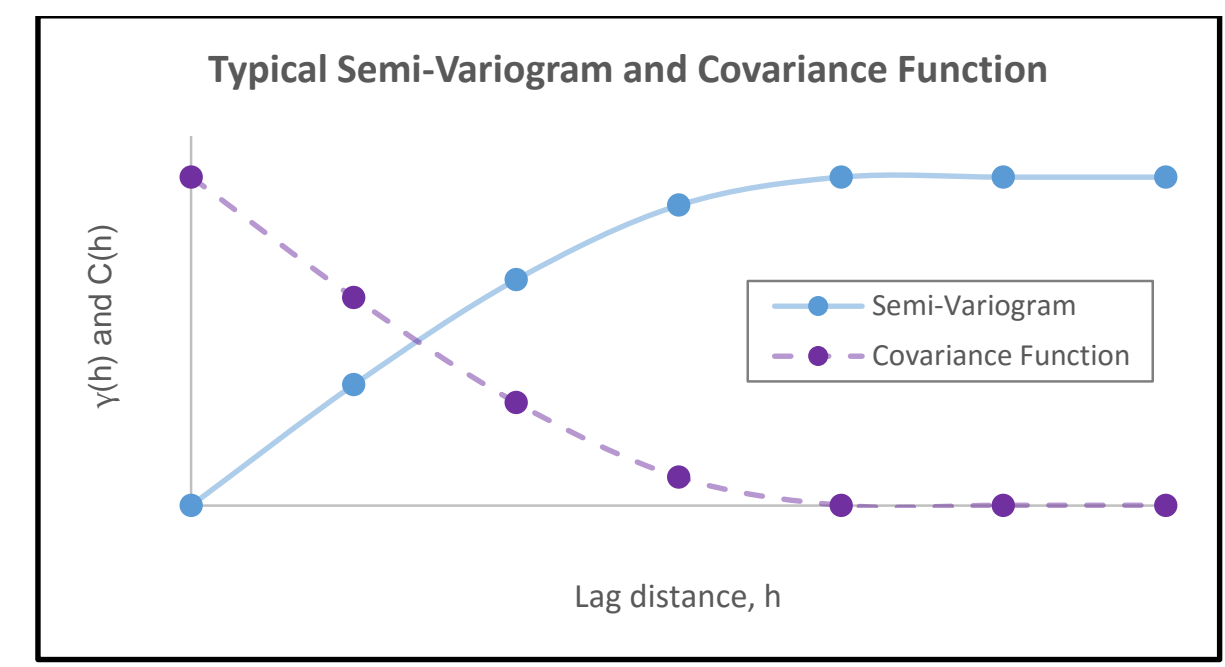

Figure 2.11 Typical shape of the semi-variogram and covariance function

These equations help to quantify how the data changes with distance, but they only allow a description for a discrete amount of lag distances. For example, if there are no data pairs that are close to being separated by 10 units, then no value can be calculated 
for this lag. Because of this, the natural progression is to create a model that fits the spatial continuity functions so that all lag values can be analyzed.

\subsubsection{Spatial Continuity Models}

There are not many earth science applications that are understood well enough to create a deterministic model (Stein and Stein, 2014). Because of this, it is assumed that the process(es) are too complicated to accurately model and they are instead assumed to be random processes. Although this may not be the case (usually it is multiple, complex processes that produce the values), the random assumption tends to give accurate results (Webster, 2000).

For estimation purposes, the models used to describe the spatial continuity need to be positive definite (the eigenvalues need to be positive). The derivation of this condition is outside the scope of this study, but can be referenced in Strang (1980). Among other things, positive definiteness is required because it guarantees that the estimations exist, that they are unique, and that they are stable (Isaaks and Srivastava, 1989).

Bohling (2005) describes how geostaticians use the semi-variogram more than the other spatial continuity functions primarily because it tends to filter out the influence of a spatially varying mean value. The covariance function requires second-order stationarity of the variable (Oliver and Webster, 2015) which means that the random process is assumed to have distributed the values with a constant mean (i.e. the average of the values is the same at every sub-location). This can be seen in the definition of the covariance function - it includes terms for the mean values and these mean values are not a function of $x$ (they are assumed to be constant). This allows the covariance function to rely solely on the lag distance and not on location. The semi-variogram only requires that 
the first differences, $V(x)-V(x+\boldsymbol{h})$, are second-order stationary (Bohling, 2005) which is what Matheron (1963) referred to as intrinsic stationarity. This can also be seen in the definition of the semi-variogram; the only variables involved in the summation are the data pairs. Oliver and Webster (2015) describe how intrinsic stationarity assumes the expected differences of the values to equal zero;

$$
E[V(x)-V(x+\boldsymbol{h})]=0
$$

which replaces the covariance of the residuals with the variance of the differences;

$$
\operatorname{var}[V(x)-V(x+\boldsymbol{h})]=E\left[\{V(x)-V(x+\boldsymbol{h})\}^{2}\right]=2 \gamma(\boldsymbol{h}) .
$$

And this spatial relationship defines the variogram (half of this value defines the semivariogram). This is a useful replacement because it relieves the requirement for a constant mean value. Since the semi-variogram does not rely on a constant mean value, it can be defined in some cases where the covariance function cannot be defined, particularly if the semi-variogram increases without bound (Bohling, 2005).

There are many models that could be used to define the spatial continuity, but some "basic models" have been created that are positive definite and vary enough to satisfactorily fit most semi-variograms that are likely to be encountered (Isaaks and Srivastava, 1989). There are two, general types of models: ones that reach a plateau and ones that do not. This plateau is called the sill (denoted as $C$ ) and the lag distance where the model reaches the sill is called the range (denoted as $a$ ). The models that reach a sill are called transition models, and the basic transition models are the spherical model, the exponential model, and the Gaussian model (Isaaks and Srivastava, 1989). These models can be seen in Figure 2.12. 


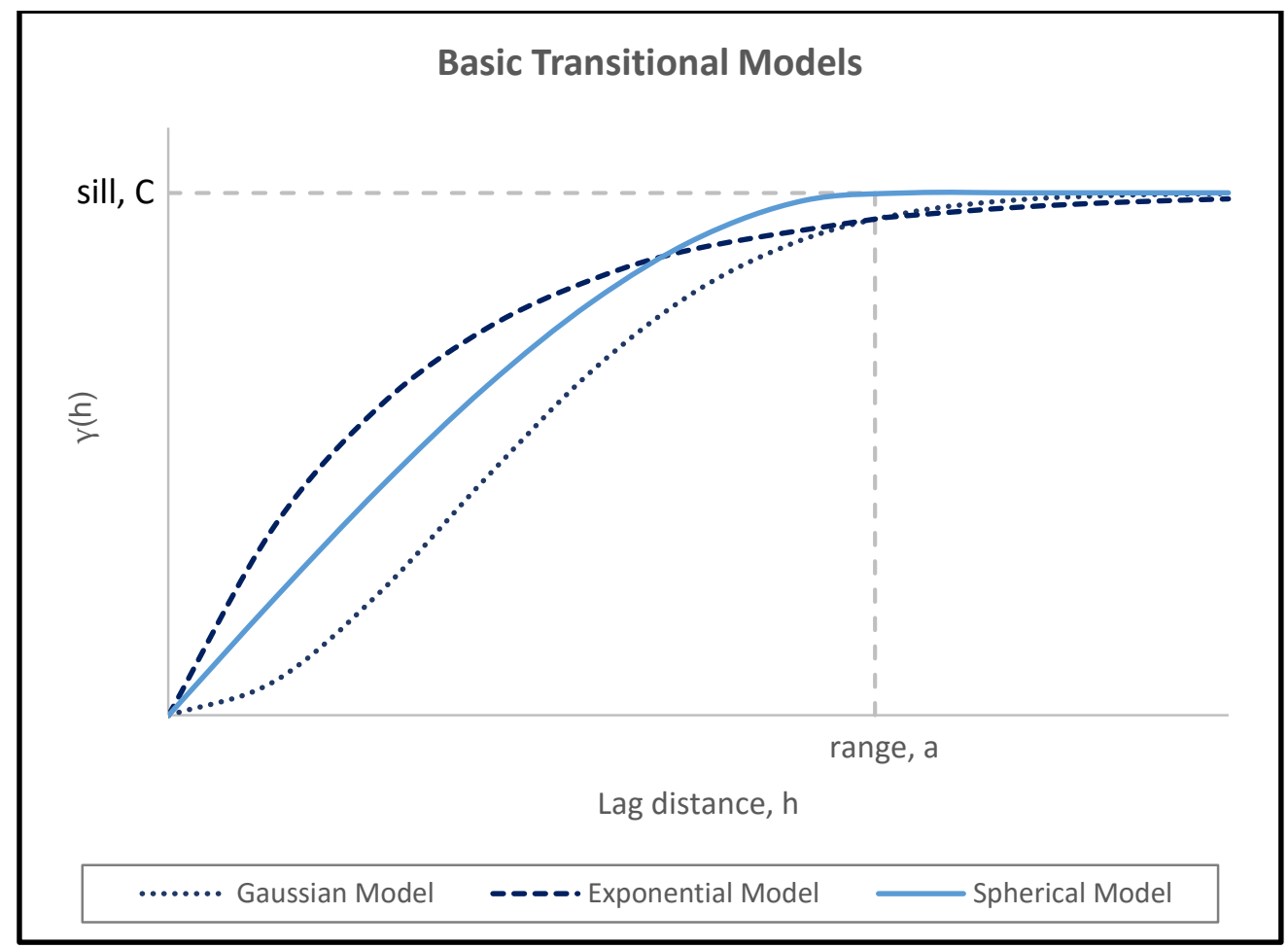

Figure 2.12 Basic transition models

The equation for the spherical model is;

$$
\gamma(\boldsymbol{h})= \begin{cases}C\left(\frac{3 \boldsymbol{h}}{2 a}-\frac{\boldsymbol{h}^{3}}{2 a^{3}}\right) & \text { when } \boldsymbol{h} \leq a \\ C & \text { when } \boldsymbol{h}>a\end{cases}
$$

the one for the exponential model is;

$$
\gamma(\boldsymbol{h})=C\left[1-\exp \left(-\frac{3 \boldsymbol{h}}{a}\right)\right]
$$

and the one for the Gaussian model is;

$$
\gamma(\boldsymbol{h})=1-\exp \left(-\frac{3 \boldsymbol{h}^{2}}{a^{2}}\right)
$$

A function that is second-order stationary will reach a sill and this sill defines the a priori variance, $\sigma^{2}$, of the random function that is assumed to have created the values 
(Oliver and Webster, 2015). In other words, it represents the variance for the distribution of values at the site. And the lag distance that corresponds to the sill (the range) defines the limit of spatial correlation. Values with lag distances greater than this are spatially uncorrelated or independent (Oliver and Webster, 2015). Notice that the exponential model and Gaussian model only reach the sill asymptotically. These models do not technically have a range since the model never reaches the sill, but a practical/effective range is used in practice that is defined as the lag distance at $95 \%$ of the sill value (Isaaks and Srivastava, 1989).

Unbounded models are those that do not reach a sill, and basic unbounded models are the power model and the linear model (seen in Figure 2.13). The equation for the power model is defined as;

$$
\gamma(\boldsymbol{h})=g \boldsymbol{h}^{\beta} \quad \text { for } 0<\beta<2
$$

where $g$ describes the intensity of the variation and $\beta$ describes the curvature. The limits 0 and 2 are excluded because $\beta=0$ creates constant variance for all lag distances and $\beta=2$ creates a parabolic function which means that the process is not random (Oliver and Webster, 2015). If $\beta=1$, the model becomes a linear model with $g$ representing the slope of the line. Because the models are unbounded, the covariance function and correlogram do not exist, but the semi-variogram does exist and fulfills Matheron's (1965) intrinsic hypothesis. This is the intrinsic stationarity discussed earlier in the chapter where the semi-variogram is not restricted by a constant mean value. 


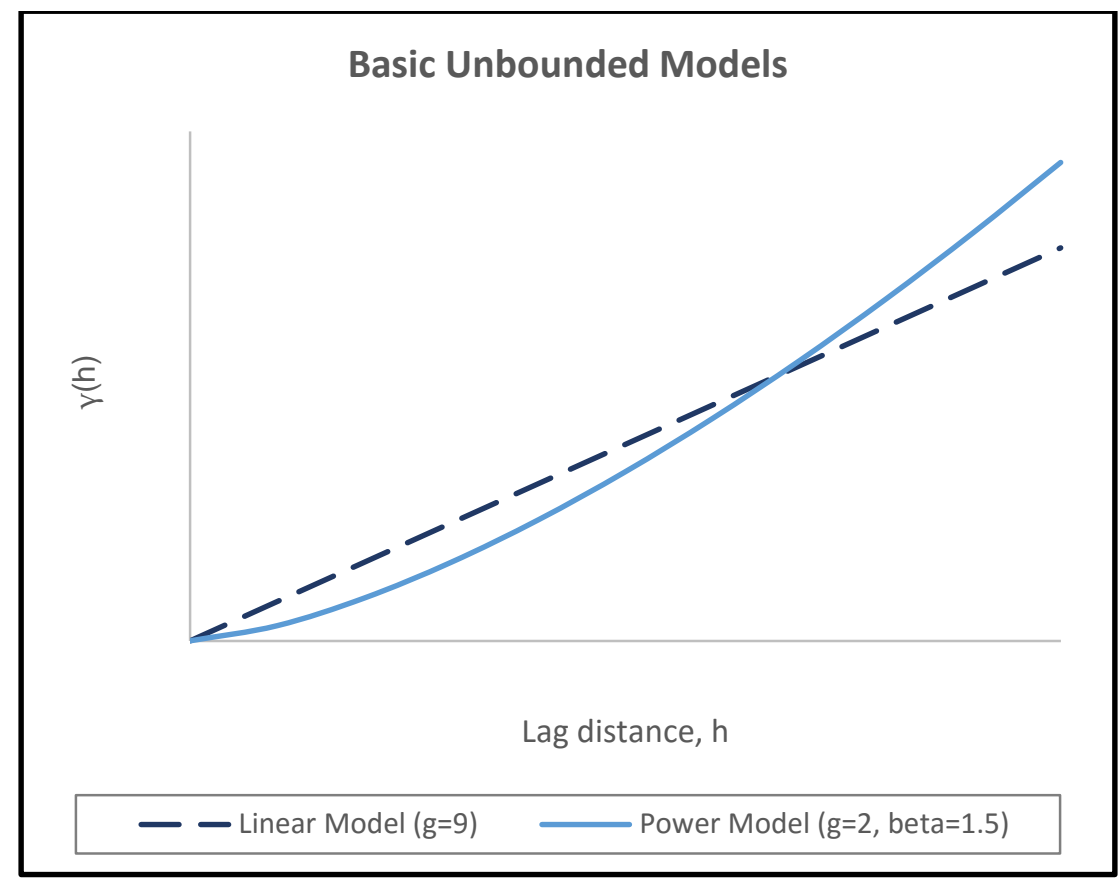

Figure 2.13 Example of basic unbounded models

All of the models shown thus far have intersected at the origin. Although the semi-variogram value for $h=0$ is strictly 0 , values at very small lag distances may be larger than 0 which creates a discontinuity. This implies that neighboring data have an average variance that is significantly larger than 0 . This can be from several factors; some examples being random error, variation over distances less than the sampling interval, and measurement error (Oliver and Webster, 2015) which all show the unpredictable component of the values. This jump is called the nugget effect. Oliver and Webster (2015) describe that the term "nugget" came from gold mining because gold nuggets appeared to occur at random and independently of one another. They created an uncorrelated component because the gold content did not relate to the neighboring sites. This effect can be modeled by adding $\omega_{o} \gamma_{o}(\boldsymbol{h})$ to the model where $\omega_{o}$ is the height of the discontinuity and $\gamma_{o}(\boldsymbol{h})=\left\{\begin{array}{lr}0 & \text { if } h=0 \\ 1 & \text { otherwise }\end{array}\right.$. This translates to being 0 at the origin and a constant value otherwise. 
When deciding which model to fit, it is best to use the one that gives the smallest sum of squared residuals (Oliver and Webster, 2015). If the sample semi-variogram appears to have a sill, then the behavior of the values near the origin can help select a model as well. If the points near the origin appear parabolic, this resembles the Gaussian model. If a line drawn from the first few points intersects the sill value at two-thirds of the range, this resembles a spherical model. And if a line drawn from the first few points intersects the sill value at one-fifth of the range, this resembles an exponential model (Isaaks and Srivastava, 1989). An example of this can be seen in Figure 2.14.

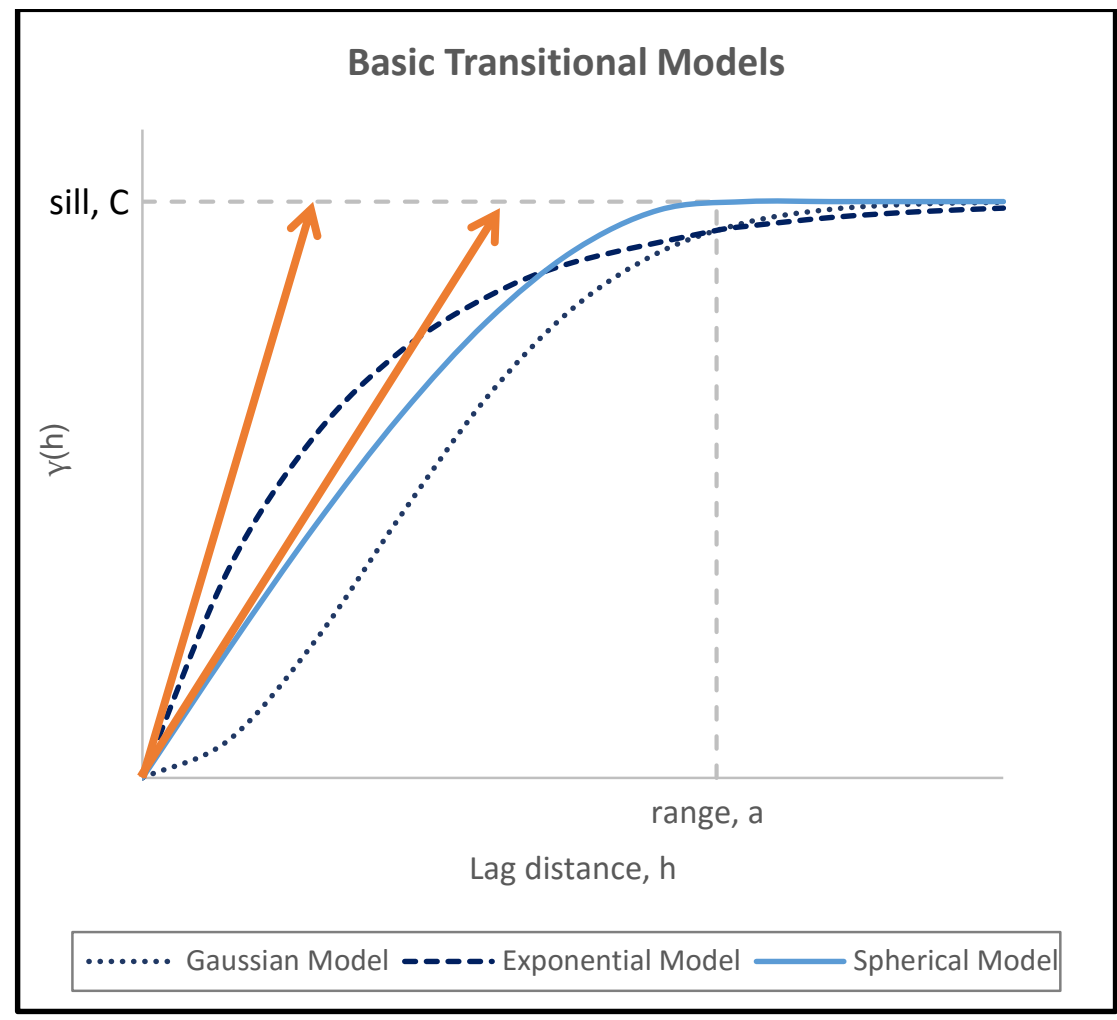

Figure 2.14 Deciding on a model from the first few points in the semi-variogram

\subsubsection{Limitations}

A major limitation is the presence of a trend in the data. The process that created the values is assumed to be random, but a trend indicates a non-randomness that will give inaccurate values if not accounted for (Oliver and Webster, 2015). This violates the 
stationarity assumption because the mean value varies as a function of distance. Instead of the variance being estimated from $V(x)=\mu+\varepsilon(x)$, a trend implies that $V(x)=$ $\mu(x)+\varepsilon(x)$ where $V(x)$ are the values of interest, $\mu$ is the mean of the process, and $\varepsilon(x)$ is the spatially-correlated, random residual (Oliver and Webster, 2015). Trends may be fairly obvious if the values are plotted, but a hint that one may be present is if the semivariogram steadily increases without bound which creates negative correlation between variables separated by large lags (Bohling, 2005). If the trend is defined and a model is fit to the data, the trend can be subtracted from the data and spatial continuity analysis can be completed on the residuals (Bohling, 2005). Data sampled in small ranges (around 20 meters) is usually safe to assume that no prevailing trend will be present (Clark, 1979).

Additional limitations that could affect accuracy is the sample size and the sample interval. If too few data is sampled, the average values of the experimental semivariogram are less likely to align with the true semi-variogram and there will be more variation in these semi-variogram values. This was studied by Webster and Oliver (1992) and they concluded that a survey should aim for 150 data points with 100 data being a minimum. The sampling interval is important because, if too large, it may not capture enough points inside the range of the model. If the data plateaus, this would lead to a model appearing to be a pure nugget effect (a flat line because there are no data pairs to describe the model at smaller lag distances). Oliver and Webster (2015) recommend a sample interval that allows at least five estimates of $\gamma(\boldsymbol{h})$ before the range. Viewing semi-variogram models of similar sites can help to estimate an approximate range so that the sample interval can be estimated. 


\section{Chapter 3: Testing Methods}

The particle motion from propagating seismic waves is the information we want to capture. This data is collected using a set of receivers and an acquisition system, and multiple receivers allow the wave propagation to be recorded in both time and space. These recordings can then be used to estimate the properties of the underlying geologic material. The following sections outline what equipment is needed, how the equipment is setup, how to acquire the data, how to process the data, and then how to model the data.

\subsection{Equipment}

The following list details the equipment needed for passive surface wave testing, and Figure 3.1 illustrates the equipment that this study used while testing (with numbers corresponding to the list below).

1. Laptop with Vibrascope and Geogiga Seismic Pro installed

2. Seismic recording system (DAQ Link III Seismograph)

3. $12 \mathrm{~V}$ battery for data acquisition (DAQ) system

4. Ethernet cable

5. DAQ power cable

6. 12-channel geophone cable

7. $4.5 \mathrm{~Hz}$, single-channel, vertical-motion geophones $(\times 12)$

8. Measuring tape (minimum of 10 meters)

9. Metal stake 


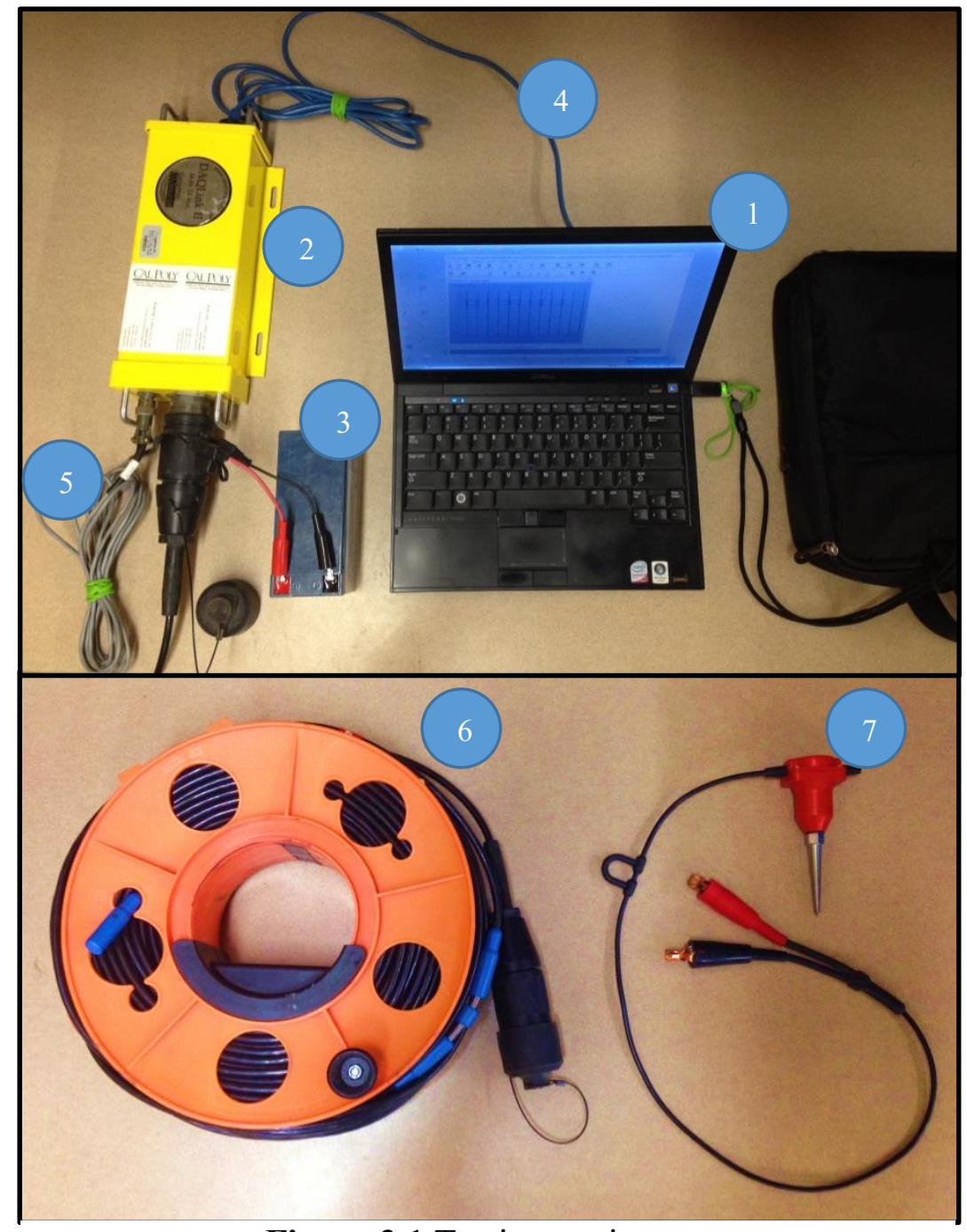

Figure 3.1 Testing equipment

It should be noted that the receivers have inherent limitations. The receivers in the array can only accurately record waves that are above their natural frequency (Lai, 2005) which is the resonant frequency of the oscillator. The amplitude of the waves will be greatly attenuated in the recordings for frequencies below this natural frequency. Since this study uses $4.5-\mathrm{Hz}$ geophones, any wave with a frequency lower than $4.5 \mathrm{~Hz}$ will not be accurately measured. 


\subsection{Field Setup}

Although there are many different array types, this study uses a 10-meter-diameter, circular array with 12 geophones. The reason for using this type of array is empirical; past experience and trial-and-error have shown that, compared to other array types, this geometry gives adequate resolution and depth. The performance of an array depends on the array geometry and the properties of the wave field, but there is no universal agreement on the array design (Foti et al., 2014).

This study set all the 10-meter, circular arrays along a straight line with each consecutive array overlapped by 5 meters. The approximate locations of each of these arrays can be seen in Figure 3.2. Every fifth array in the figure is highlighted to reduce clutter and there is a gap of three arrays due to a hill on the athletic field. Each array was labeled based on its distance from a 4-foot, metal stake on the site. This stake is at the center of the fifth array and is labeled in the figure.

Setting up in the field begins by determining the line (array azimuth) and a starting location. The array azimuth in Figure 3.2 was chosen based on the geology, topography, and constraints of the site. In choosing the azimuth, the objective was to create a long array on flat ground that sampled similar material. The starting/reference location was chosen based on logistics and ease of access at the site. 


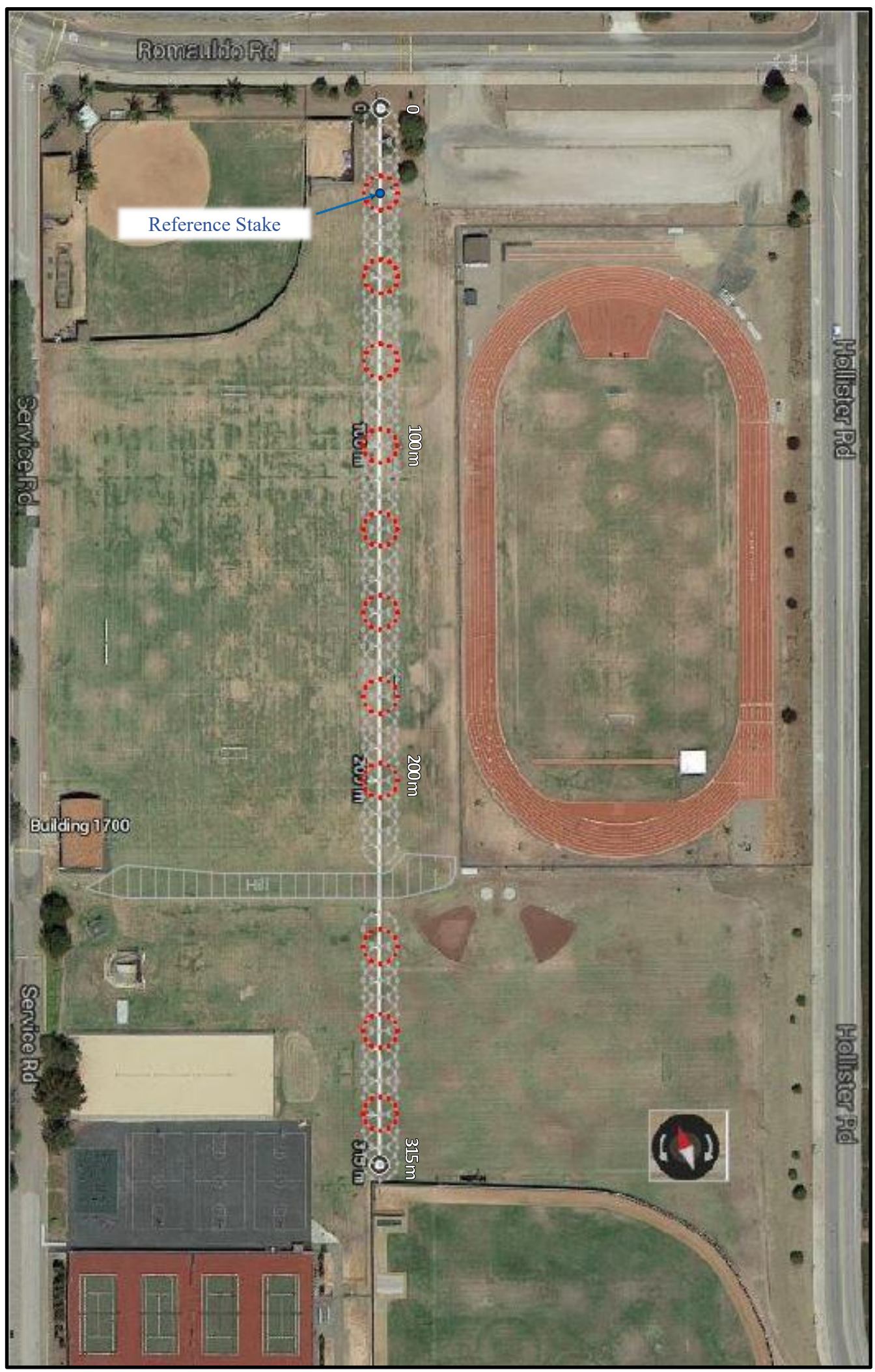

Figure 3.2 This study's array line at Cuesta athletic fields 
Since the array is circular, it was easiest to anchor the measuring tape to the center of the array with a stake and rotate radially around the stake to create a perfect circle. The approach we used to place the geophones was to start with the cardinal points of the circle. Using the measuring tape, 4 geophones were placed at each cardinal point - making sure that two of the cardinal points were in line with the array azimuth. Since there are 12 total geophones, the four quadrants that were just created needed to be divided into thirds in order to accommodate the remaining 8 geophones. Estimating these locations visually or measuring the distance by pacing around the circle proved to be sufficient for this study. Each geophone was pushed into the ground so that it had intimate contact with the soil. If the top soil was too stiff to place the geophones by hand, we would hammer a stake partway into the soil to create a void for the geophone spike. This was sufficient because ground coupling and the tilt of the geophones only minimally influence the data (O'Neill, 2003). Once all the geophones were set, the geophone cable was laid around the circle and the geophones were attached to their respective leads. Since we had multiple arrays, we made sure to keep the geophone numbering in the same orientation (e.g. the first and seventh geophones were always along the array azimuth). The cable was plugged into the seismic recording system (DAQ Link III seismograph), and this system was attached to both the $12 \mathrm{~V}$ battery and the laptop via the power cable and the Ethernet cable, respectively. After these steps, the array is ready for data collection in Vibrascope. An example of what the finished array should resemble can be seen in Figure 3.3. 


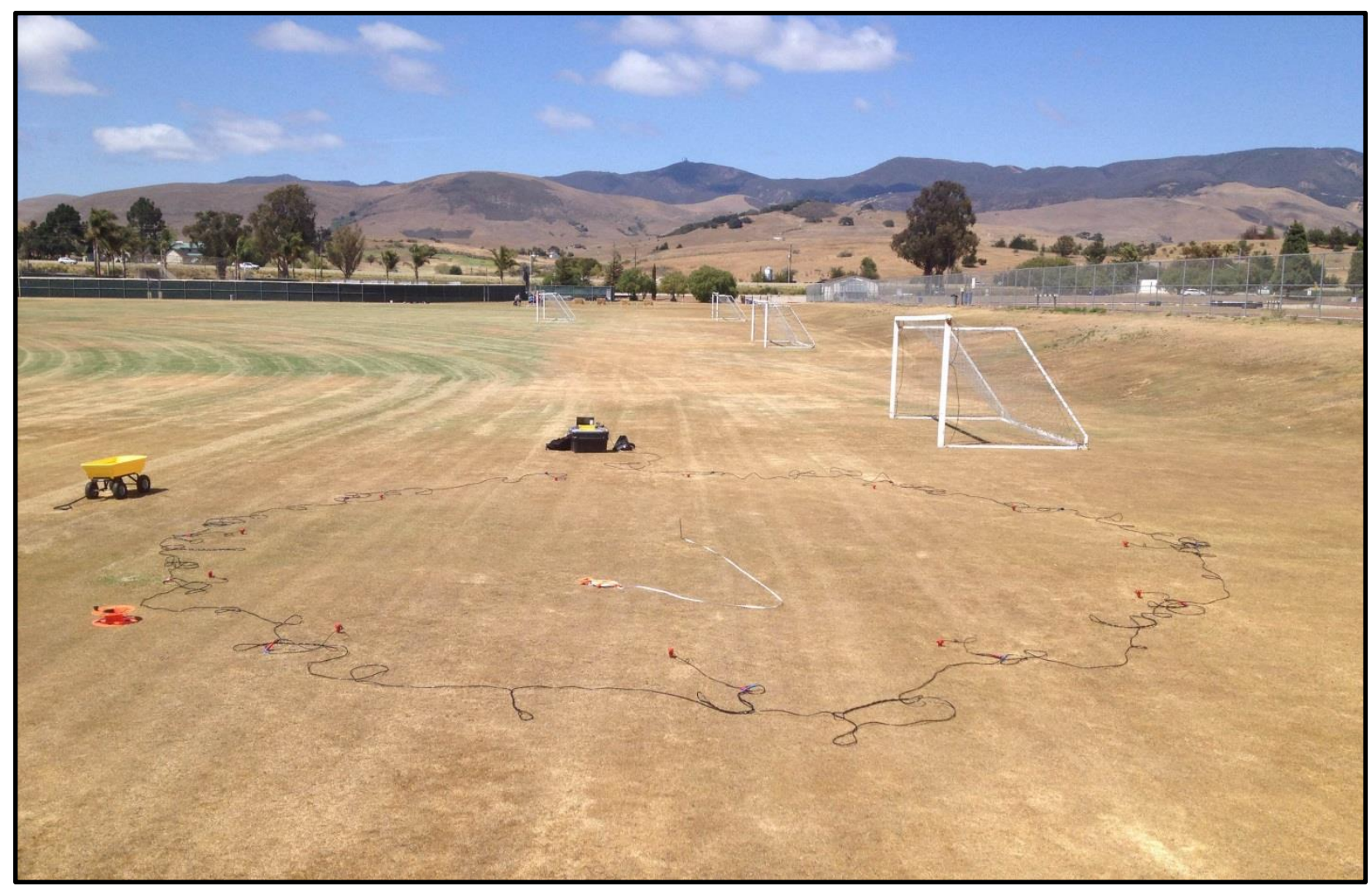

Figure 3.3 A visual of this study's array setup

\subsection{Acquisition of Experimental Data}

In Vibrascope, it is best to create a new project for each array location so that organization and accessibility of the files remains simple. This can be done by selecting "New Project" from the "File" drop-down menu. In this "New Project" window, as seen in Figure 3.4, input the project name and the project location. Vibrascope will create a new folder with the specified project name and the folder will be placed in the specified project location. 


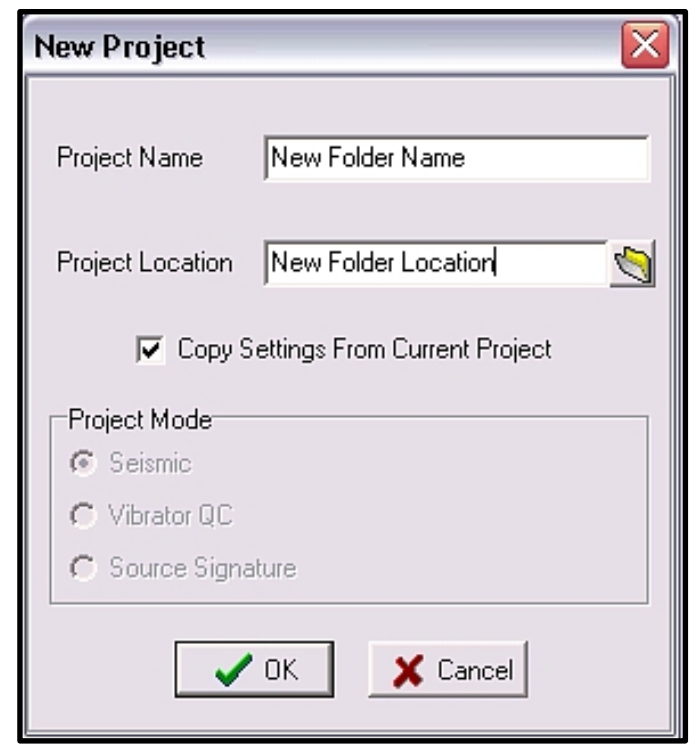

Figure 3.4 New Project window in Vibrascope

Once the new project has been created, the recording parameters can be verified and adjusted in DAQ Setting. This can be found by selecting the "Options" drop-down menu and clicking "Device", or by pressing the "Device" icon $\underline{\text { Device }}$ in the ribbon. In this DAQ Setup

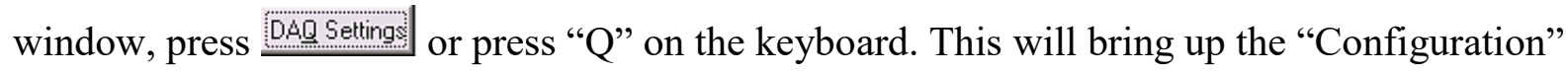
window as seen in Figure 3.5. In the acquisition tab of this window, be sure that all 12 channels are selected in the "On" column, that each channel has the "Type" as generic, the "Gain" as 1 , the "DC Removal" as Auto at 0.0, the "Units" as V, and the "Scale" as 1. 


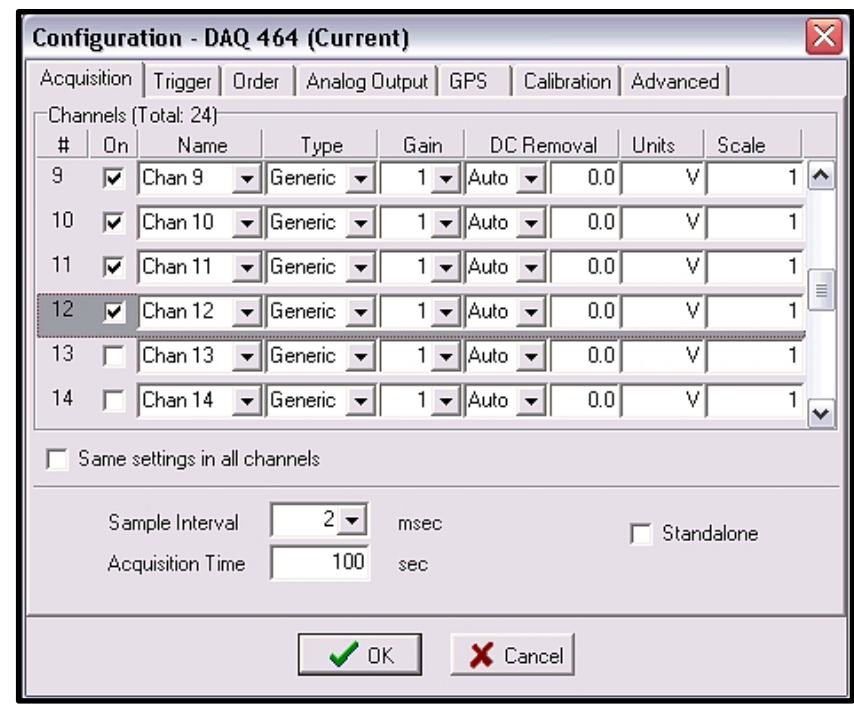

Figure 3.5 Configuration window on the Acquisition tab

For surface wave recordings intended for SPAC or f-k analysis, the "Sample Interval" should be set to 2 milliseconds and the "Acquisition Time" should be set to 100 seconds. The 2 millisecond acquisition time is because analysis is done in the frequency domain and the frequencies of interest are usually below $100 \mathrm{~Hz}$ (Foti et al., 2014). The 100-second cap is because, at the 2 millisecond sampling rate, this is the largest file that most software can handle. In the trigger tab on the Configuration window, be sure that "Auto Trigger" is selected. Once this is complete, press $\checkmark$ oK on the Configuration window and then on the DAQ Setup window to accept the settings and return to the main screen. Note that if the Configuration window did not resemble Figure 3.5, but rather resembles Figure 3.6, then "Advanced Mode" has been turned off. 


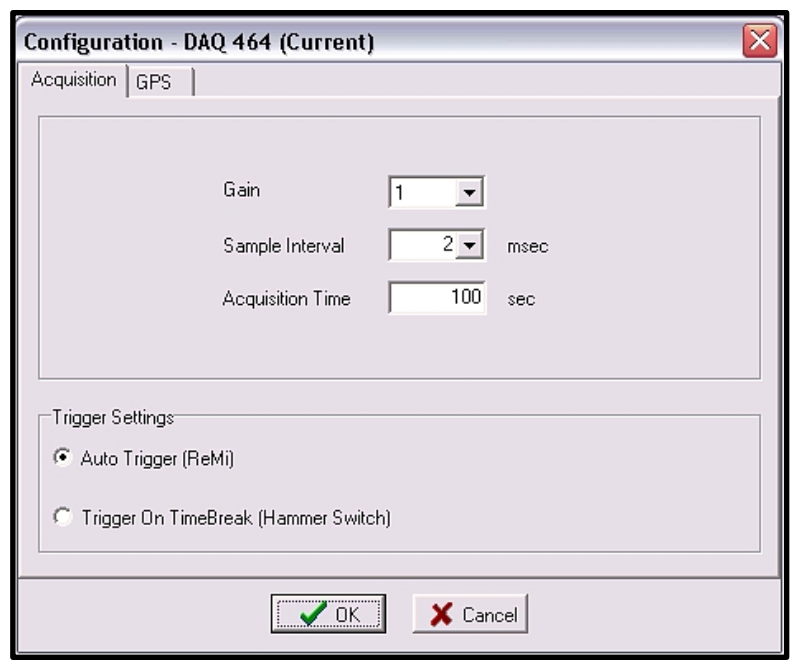

Figure 3.6 The Configuration window if "Advanced Mode" is not selected

To select "Advanced Mode", drop down the "Options" menu on the main screen and choose "Preferences." In the Preferences window, make sure that "Advanced Mode" is checked.

When the DAQ settings are correct, the data can be collected by pressing $\stackrel{\mathscr{D}}{\text { Start }}$ in the ribbon or by pressing " $\mathrm{A}$ " on the keyboard. It may prove easier to run a 10 -second recording before running the first real acquisition. This can be done by changing the acquisition time to 10 in the DAQ Settings window. This 10 -second recording should not be saved, but it allows the user to see if all the geophones are receiving data and if the seismic plot looks appropriate without having to wait the full 100 seconds. An example of an appropriate 10 -second recording can be seen in Figure 3.7. 


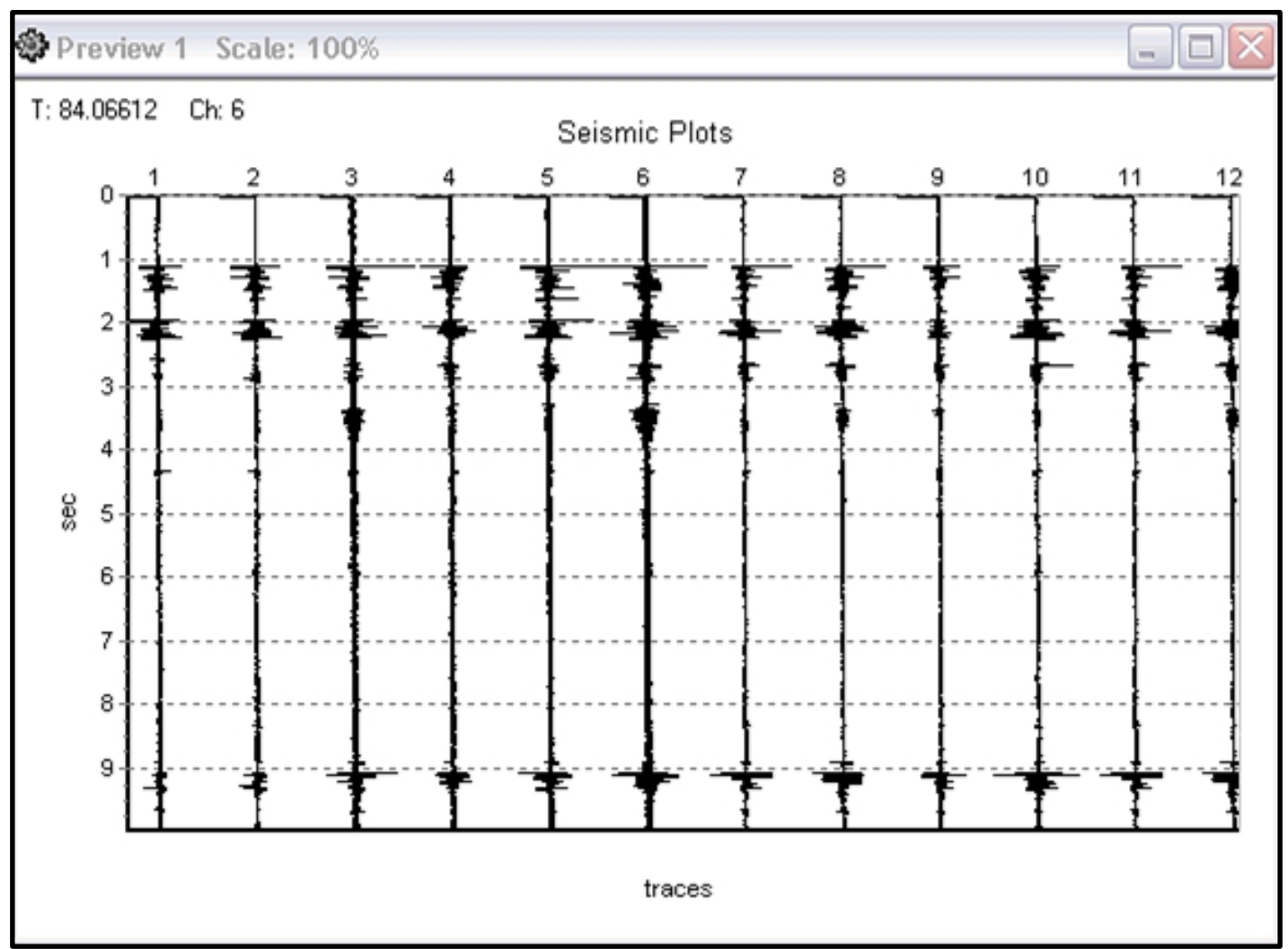

Figure 3.7 An example of an appropriate 10-second recording

If the 10-second recording seems reasonable, then the acquisition time can be changed back to 100 seconds and the first real recording can be taken. Notice that, along the bottom of the main screen, Vibrascope shows how many seconds that the recording has been receiving data after pressing start (circled in Figure 3.8). 


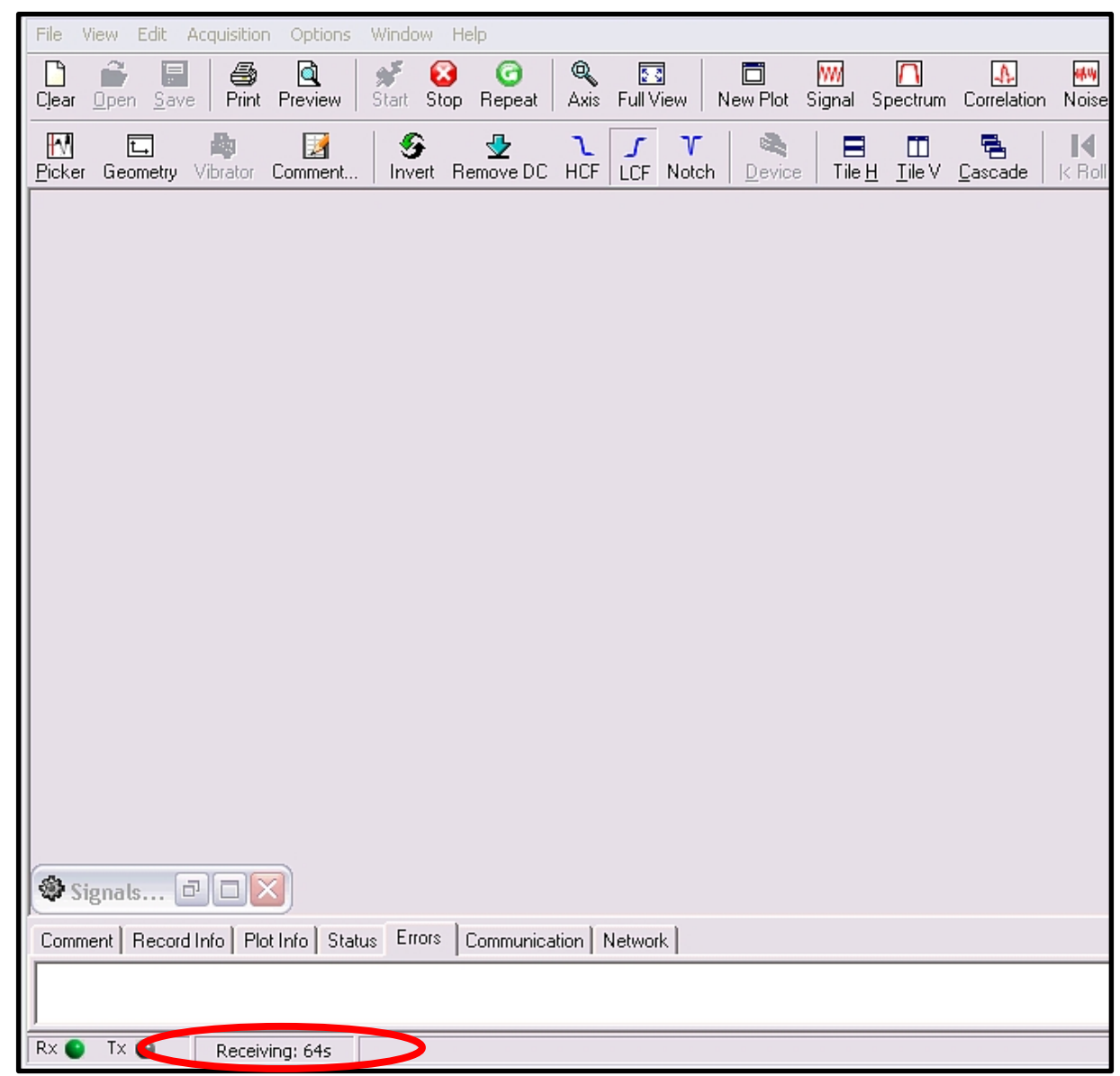

Figure 3.8 Vibrascope's indication of how long the recording has been receiving data

This can be helpful in keeping track of the time and in noting how certain seismic sources affected the data (e.g. if a concrete truck drives by the array at 40 seconds of recording, the seismic plot should reflect this). Once the 100 seconds of recording are complete, a window displaying the signal traces for each geophone should appear on the screen. It is good to check this window for anomalies, but be advised that the traces in the seismic plot are scaled to the largest amplitude that was recorded. This means that if the array recorded a wave with significantly higher amplitude (maybe from a heavy object dropping nearby), then the scale would be adjusted to this amplitude in the seismic plot and the motion of the lower amplitude waves may appear to approach zero. If there was adequate noise during the recording though, 
this scaling would just be hiding the lower amplitude waves and the use of the recording would still be appropriate.

If the recording seems suitable, then the next step is to save the recording by selecting "Save" in the "File" drop-down menu or by pressing Ctrl+S. Notice that the name on the banner of the seismic-plot window changes to reflect how many recordings have been saved in the project folder. After saving, the file can be exported into the project folder by selecting "Export" in the "File" drop-down menu. In order for the signal processing software (Geogiga Seismic Pro, 2012) to be able to read these files, they must be saved as SEG-Y Tape Format (*.sgy). Naming the file with some reference to the location makes it easier to organize and identify the files during processing. The exporting window described above can be seen in Figure 3.9.

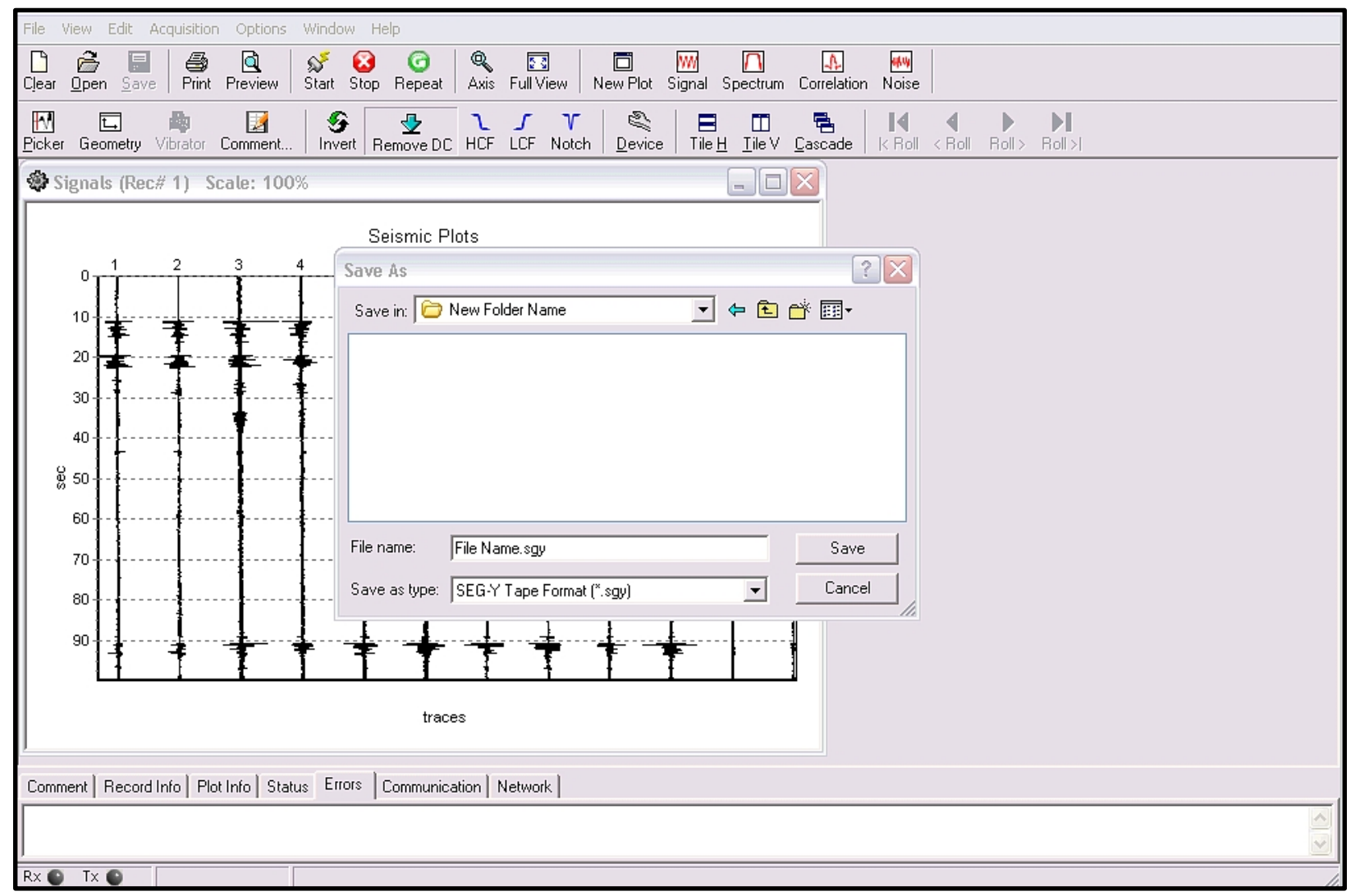

Figure 3.9 Vibrascope's export window 
Once exported, complete two more 100-second recordings by following the steps outlined above and export these into the same project folder. 100-second recordings are the largest file size that most software can handle, but more recordings are necessary to fully sample the passive/ambient noise. Three, 100-second recordings will commonly produce sufficiently accurate results. After three recordings have been completed, the array can be moved to a new location and a new project folder can be started.

\subsection{Signal Processing}

The three recordings at each location can be processed using the Surface Plus program in Geogiga Seismic Pro. Import the files by selecting "Import Seismic" from the "File" drop-down menu. Make sure that "Passive (2D Array)" is selected at the top of this window as seen in Figure 3.10, and then add the files by selecting "Add..." near the bottom of the window (as opposed to the "Add" near the Geometry Definition Group section). The file will be in the project folder that was created in Vibrascope. Highlight all three recordings and press "Open." Notice that the last digit in the "Shot No." column corresponds to which file was recorded first, second, and third.

Once the three files are loaded, the geometry for each file needs to be added. This can be done by selecting the first file and then selecting Yiew... which will bring up a "Geometry" window (seen in Figure 3.11). If there are not three columns (X, Y, and Z) in the "Receiver Locations" section then it is likely that "Passive (2D Array)" was not selected in the importing window. 


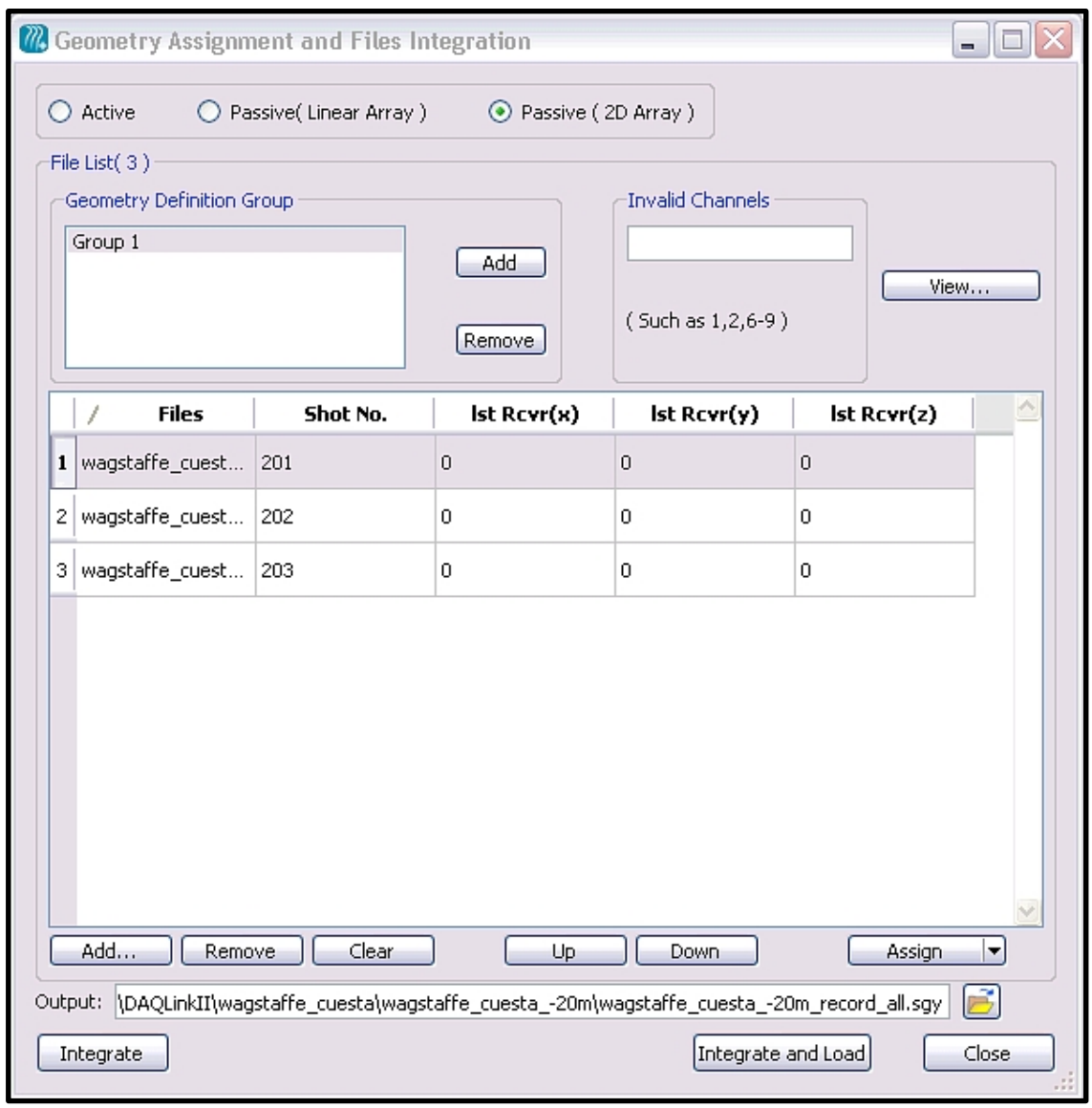

Figure 3.10 Importing seismic data in Geogiga Seismic Pro

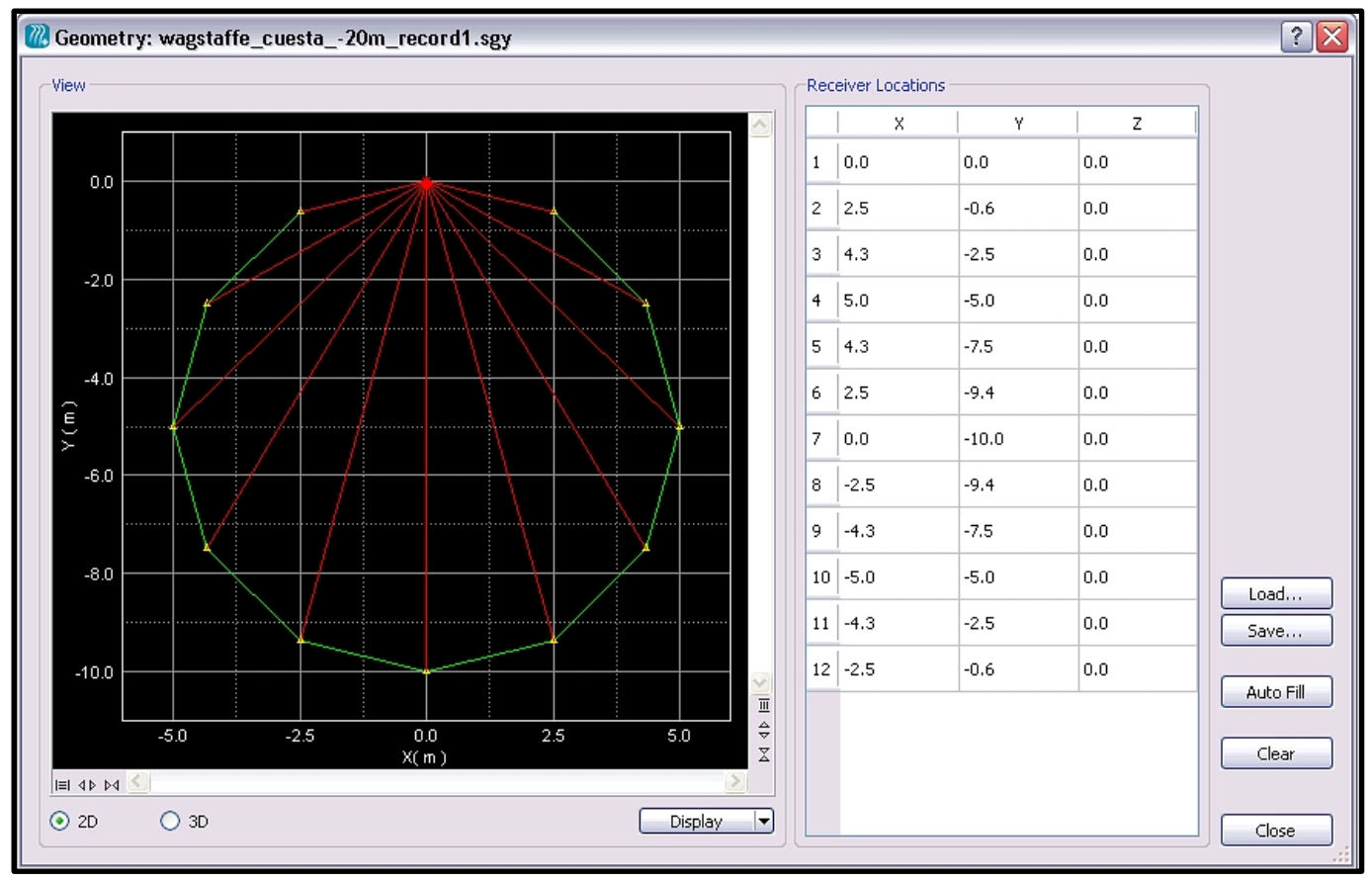

Figure 3.11 Setting geometry in Geogiga Seismic Pro 
To load the geometry, press Load... and then double-click the text file (.txt) that lists the receiver locations. It is best to save a copy of this file to the folder where the new Vibrascope projects are being saved. This will make locating the file much easier. The geometry text file for a 10-meter-diameter, circular array with 12 geophones can be seen in Figure 3.12.

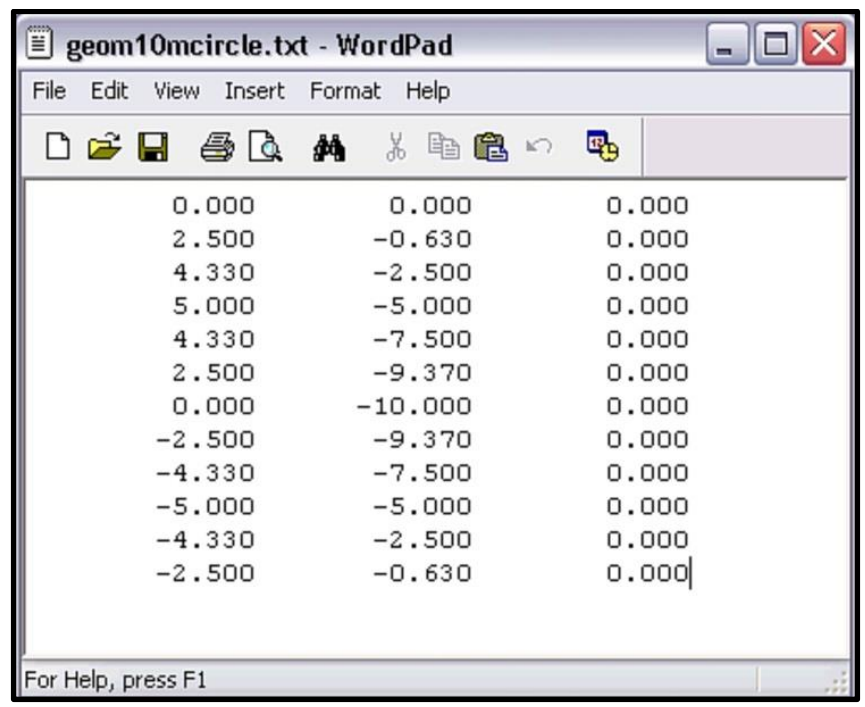

Figure 3.12 Text file with receiver locations

Double-clicking the text file will input the values into the receiver locations and the lines that connect all geophones to the first geophone should turn red. After this, press $\square$ close to exit the window. Now click on the next file and repeat the steps above to assign it a geometry. Note that simply using the arrow keys to highlight the next file will not apply the geometry to the correct recording. Also be aware that the "View..." window for the second and third recordings will show a diagram of the circular array with light blue lines, but the geometry will not actually be loaded until the text file is selected. To ensure that the geometries have been applied, make sure that the receiver locations have changed to the correct values and that the lines connecting the geophones to the first geophone have changed from light blue to red. Once all of the files have the correct geometry applied, click Integrate and Load (an error message will appear if all the 
geometries have not been applied). Geogiga will link to the "Browse Traces" option and the traces from each recording should be visible on the main screen.

The dispersion settings now need to be changed, but this cannot be done while browsing the traces; one of the traces needs to be selected so that Geogiga knows which settings to alter. To do this, select "Pick" from the "Trace" drop-down menu or press "Settings" can be selected from the "Dispersion" drop-down menu or the icon can be selected in the ribbon. In this "Dispersion Analysis" window, select SPAC as the analysis method and SPAC as the method (as seen in Figure 3.13) and then press Apply and close Note that there are other options that can be changed, but the default choices proved to be appropriate for this study. The Rayleigh wave velocity at this site did not surpass 800 meters per second and the $150 \mathrm{~Hz}$ cap on frequency proved to capture a broad enough range of waves.

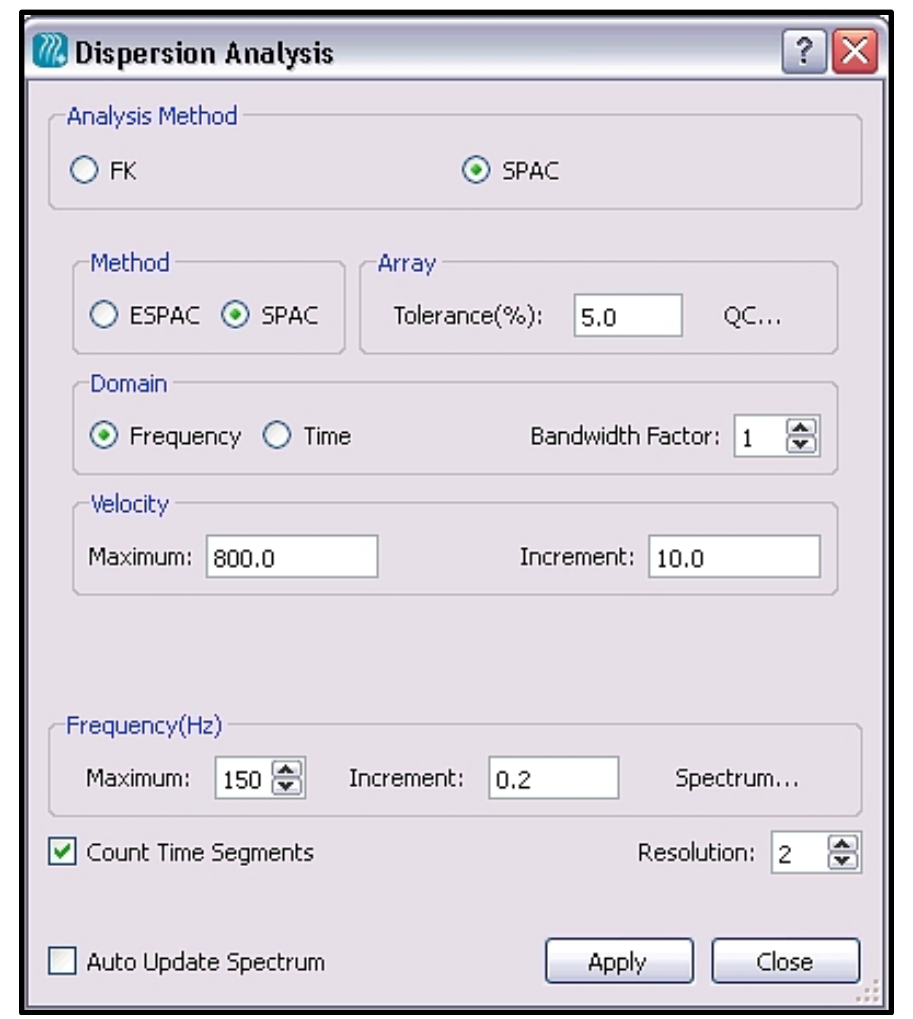

Figure 3.13 Dispersion settings in Geogiga Seismic Pro 
At this point, the three recordings can be combined. To do this, select "Combine with Other Records" from the "Dispersion" drop-down menu or select the $\sqrt{+}$ icon from the ribbon. In this spectra combination window, highlight each recording in the "Primary" column and select the "Build" option for that column. This should display each recording's dispersion spectrum as seen in Figure 3.14. Press Combine and Close to view the combined dispersion spectrum.

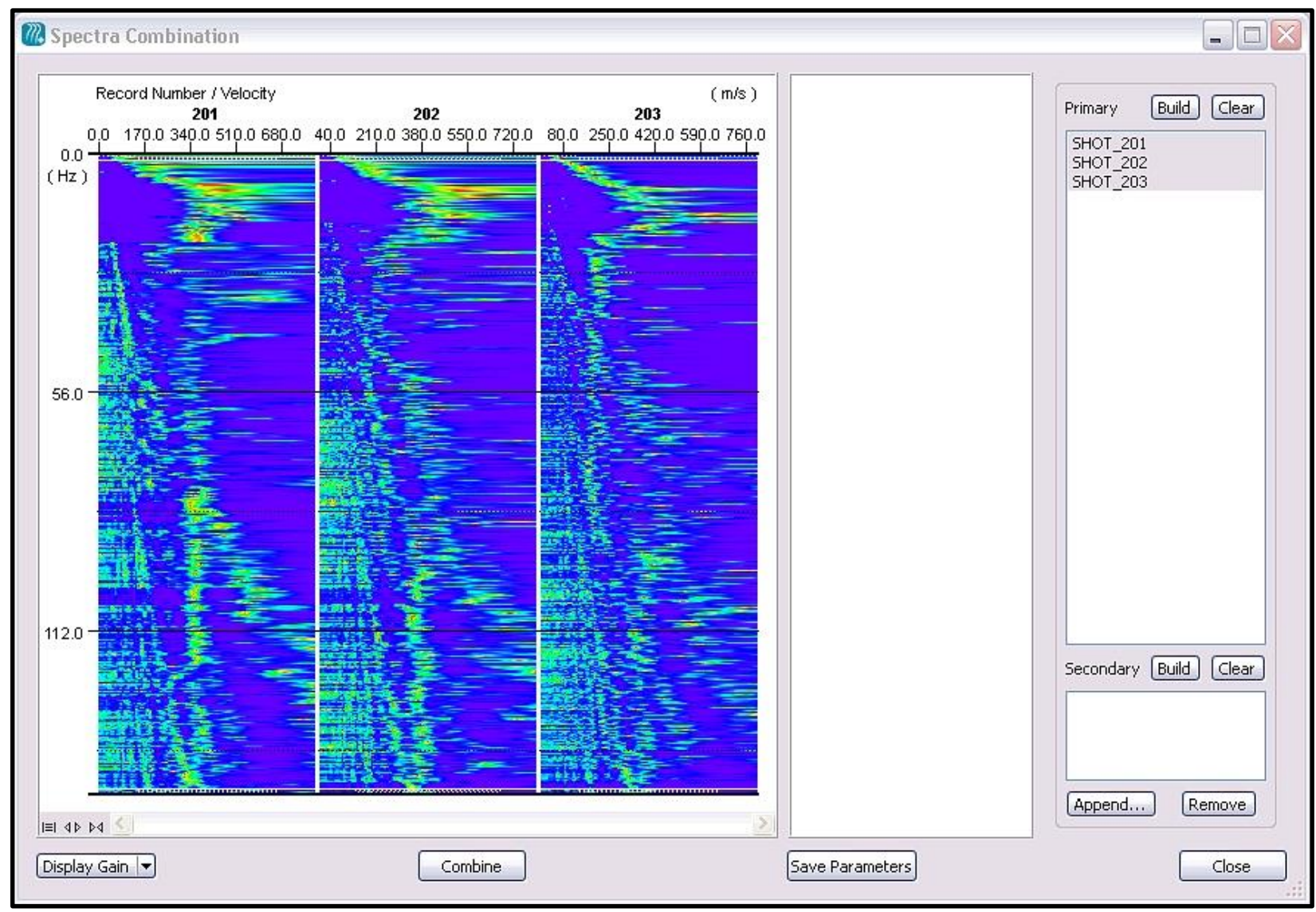

Figure 3.14 Spectra combination in Geogiga Seismic Pro to combine 3 dispersion plots Next, select points on the dispersion curve by selecting "Picking" from the "Dispersion" dropdown menu or by selecting the $\mathbb{N}$ icon in the ribbon. This will bring up a window to select the type of picking; select "Separate" instead of "Continuous" and then close the window. 
At this point, the dispersion curve can be created by using the cursor to select points along the spectrum. In normally dispersive situations, the curve should resemble an exponential decay model; pick points that appear to align with this exponential curve. As a rule of thumb for passive surface wave testing, it is better to pick points along the lower bound of this exponential curve instead of the mean value (Louie, 2001). This gives lower, more conservative estimates of the phase velocity and tries to avoid the selection of higher modal responses. For the amount of points to pick, the inversion process will work well as long the points on the dispersion curve exceed the amount of layers trying to be estimated (Rix and Leipski, 1991).

Once these points are picked, the program will automatically create a plot of the Rayleigh wave phase velocity with depth. The depth in this plot is not a direct measure, but rather is calculated from an approximation that assumes that the sampling depth corresponds to half of the wavelength (Rix and Leipski, 1991). An example of the picking and of the corresponding windows can be seen in Figure 3.15. Notice that this curve deviates from the exponential model at higher velocities due to a stiff layer near the ground surface. There is also a blue line in the central window that corresponds to the apparent shear wave velocity values. These values are not found through inversion, but rather through an equation that relates Rayleigh wave velocity to shear wave velocity. This line can be included by clicking the "Curves" drop-down menu, selecting "Dispersion Curve Display", and then checking the box for Apparent Vs. 


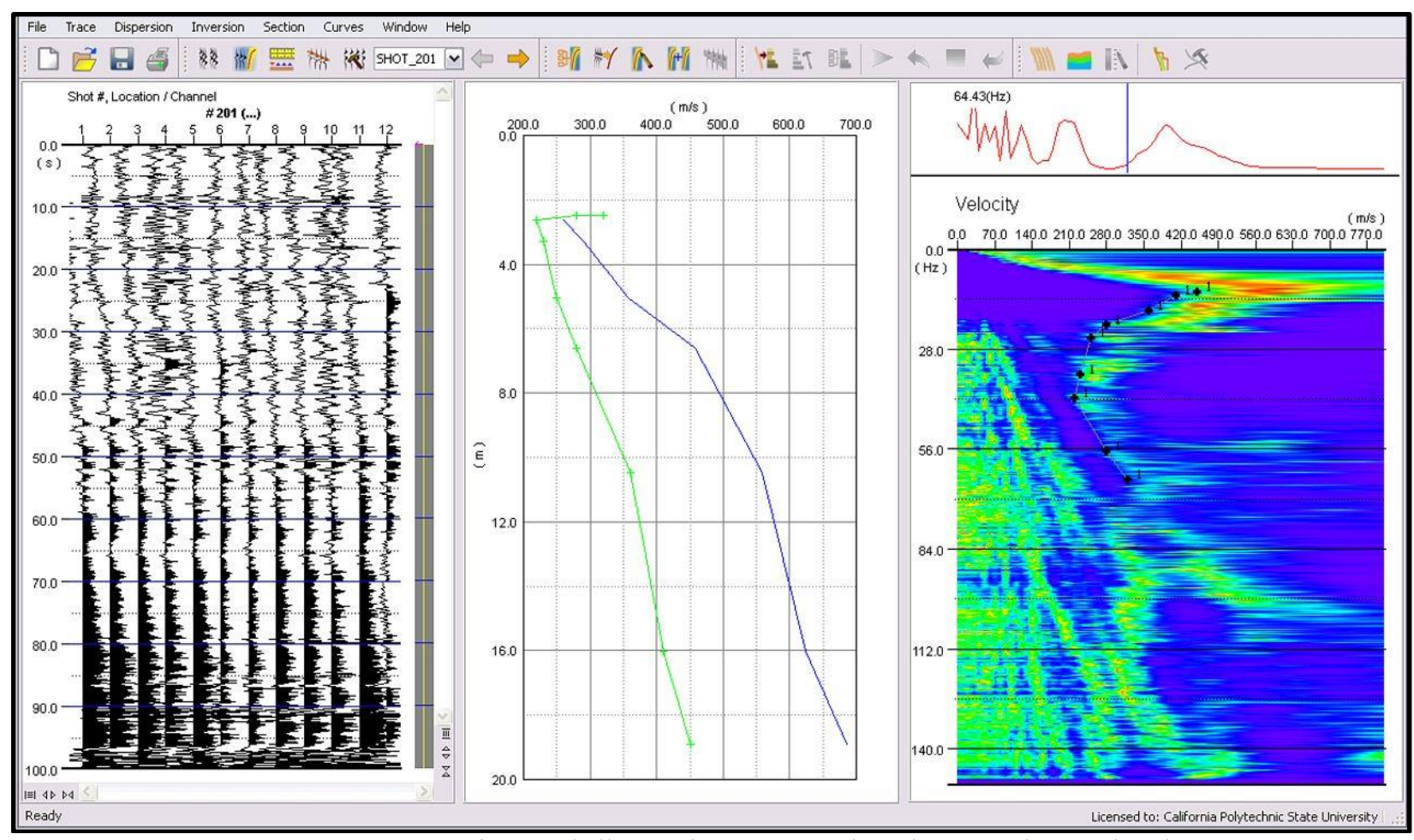

Figure 3.15 Trace, H/V, and dispersion curve plots in Geogiga Seismic Pro

The final step is to save the images of the plots. This can be done by selecting "Save Image" from the "File" drop-down menu. The "View List" column shows which plots can be saved. Highlight the desired plot, name the file, and press Save . This window can be seen in Figure 3.16. Note that, in order to save the image in JPG format, the "Files of Type" drop-down menu needs to be changed to "All files $(*)$ " and the file name should end with ".jpg". 


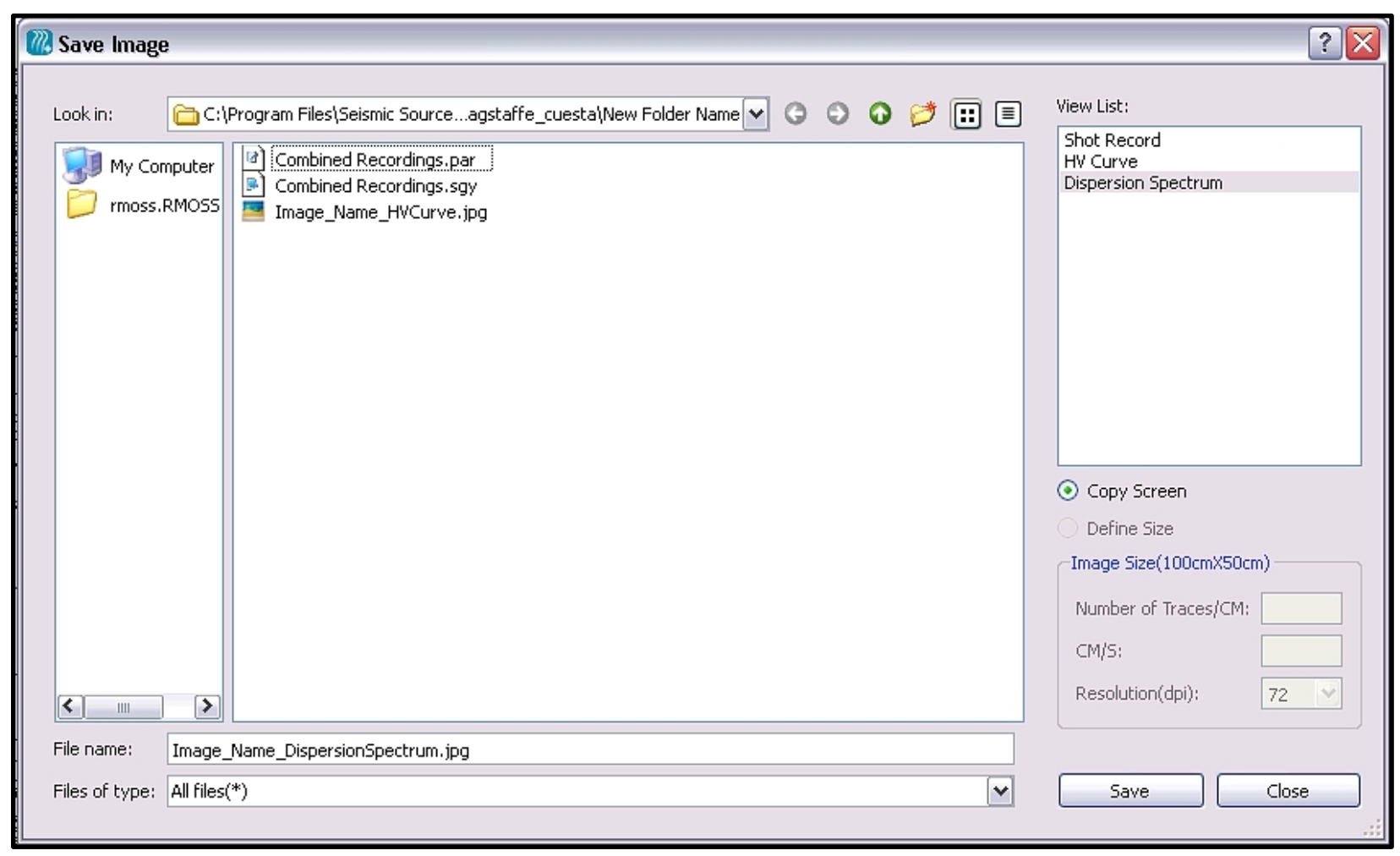

Figure 3.16 Saving an image in Geogiga Seismic Pro

\subsection{The Inversion Process}

After picking points on the combined dispersion spectrum, the shear wave velocity profile can be created by carrying out an inversion process. Geogiga Seismic Pro has preset algorithms that will carry out this process, but the solution to the inversion problem is not unique (Foti et al., 2014). Many different dispersion curves may correspond to a given shear wave velocity profile. A priori information such as nearby borings, past surveys, or geology maps will create good expectations for depths of layers and shear wave velocity values which can help to mitigate the nonunique solution.

To start the process, follow the steps outlined in the Signal Processing section (above) so that the points on the combined dispersion spectrum are selected. Through forward modeling, it will approximate a shear wave velocity profile that models the selected dispersion curve. The process tries to match the points on the dispersion curve to an estimated model through a least 
squares technique. Therefore, it is important to pick a dispersion curve that is representative. An example of this can be seen in Figure 3.17, where the same dispersion spectrum is shown but Figure 3.17 (a) shows points that were poorly selected and Figure 3.17 (b) shows points that are more reasonable for this site. Reasonable curves are typically ones that are normally dispersive since many sites fit into this category, but sites with high-velocity layers overlying lowervelocity layers should reflect this within the dispersion curve. For example, the dispersion curve in Figure 3.17(b) shows a higher-velocity layer at shallow depths. For a discussion on normally dispersive behavior and how dispersion curves vary outside of this behavior, please see Section 2.1.3. The main concern is that appropriate curve should be based on the geology of the site, and inconsistent curves can be avoided if these expectations are known.

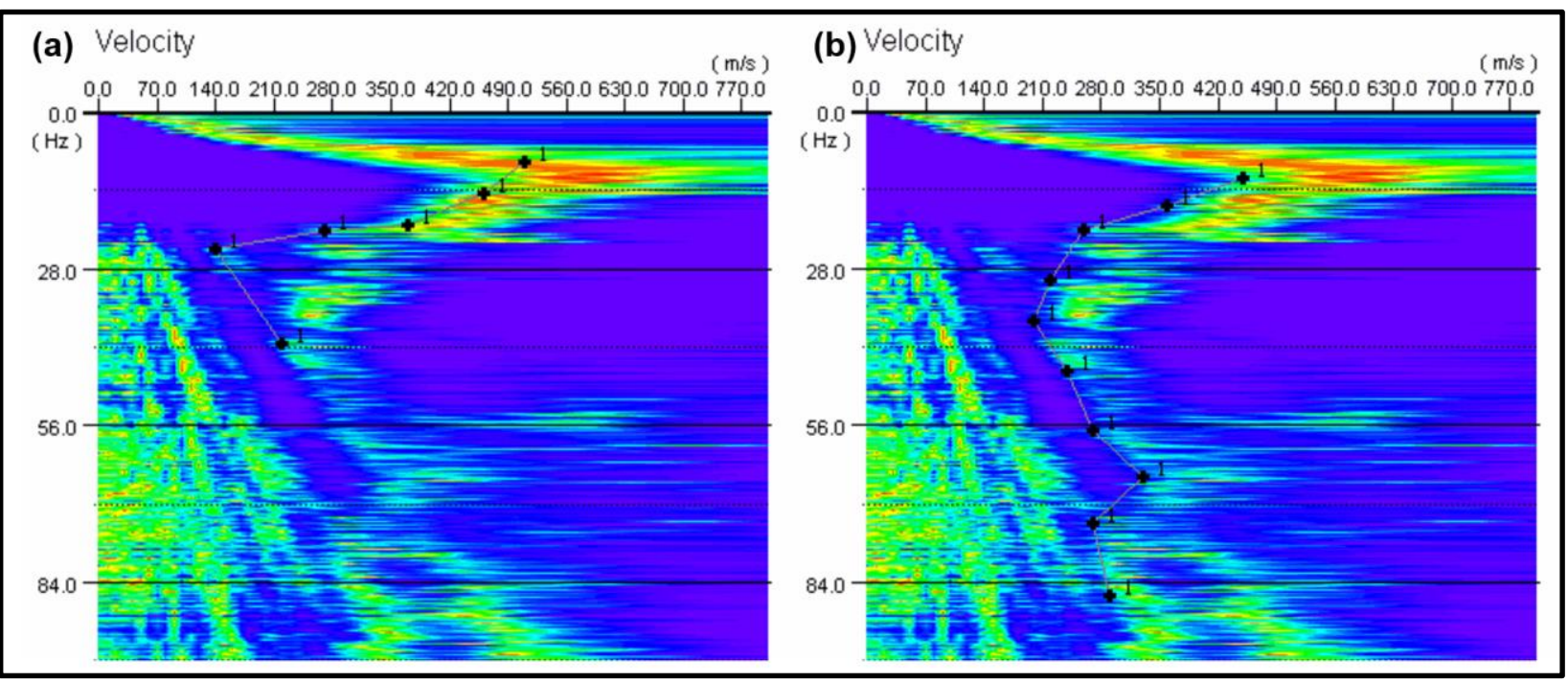

Figure 3.17 (a) Poor dispersion curve picking, and (b) appropriate dispersion curve picking

Once a reasonable curve is picked, click on "Select" from the "Inversion" drop-down menu to indicate that those points are the ones to use for the inversion. Then click on "Autobuild Initial Model" from the same "Inversion" drop-down menu. This will bring up three windows one showing the initial shear wave velocity profile from the inversion, one showing the dispersion curve that the process is trying to model, and one showing the error between the 
selected dispersion curve and the modeled dispersion curve. The default settings only allow the shear wave velocities to vary in the velocity direction. To allow the velocity and the depth to vary in the inversion iterations, click on the "Inversion" drop-down menu and select "Parameters." Then check the area for "Velocity and Depth" as seen in Figure 3.18.

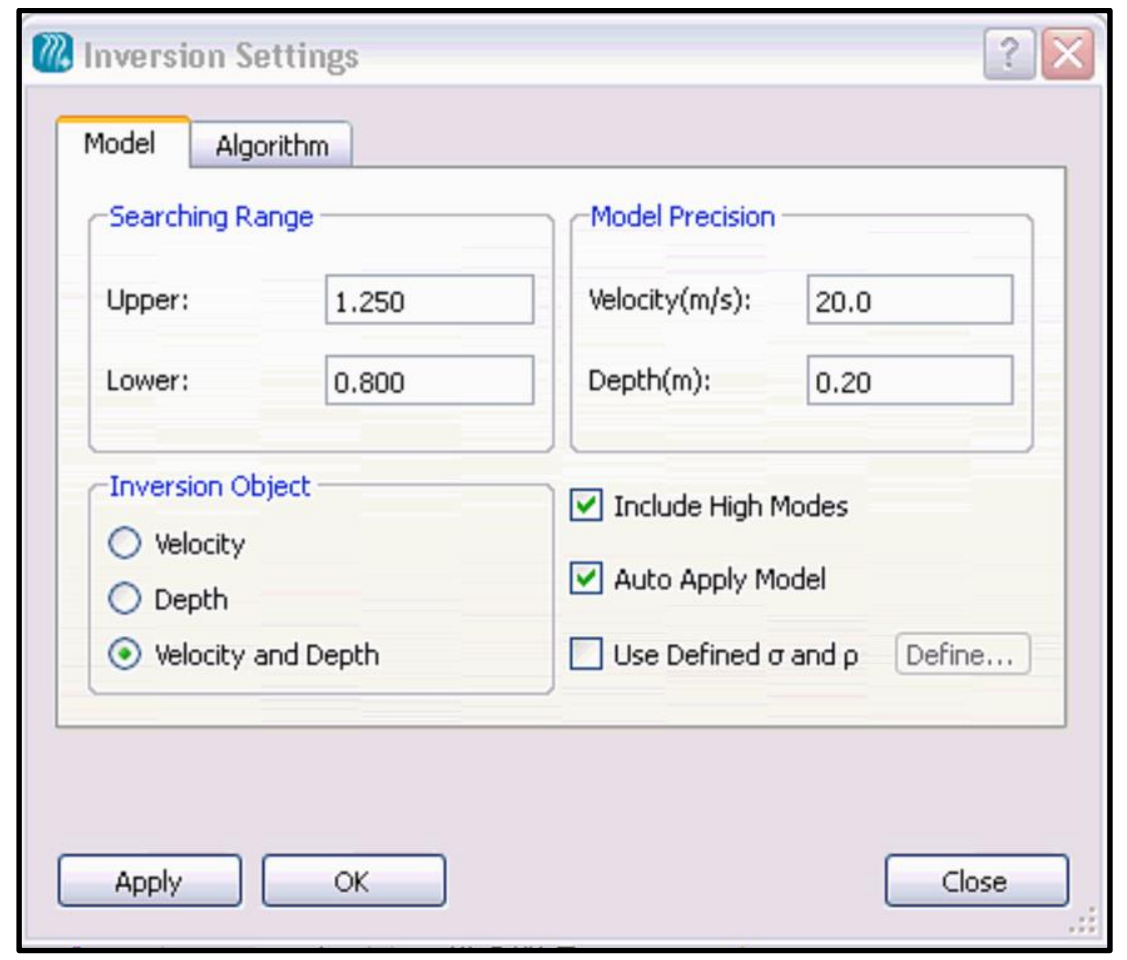

Figure 3.18 Allowing the inversion process to vary by velocity and depth

Since there is no unique solution to this inversion problem, creating the "correct" model is subjective and cannot fully be known unless confirmed by other methods. Because of this uncertainty, the way to create a model that has the most likelihood of being representative of the site is to minimize the error between the observed and the modeled dispersion curve, and to use good judgment about the values the inversion process generates. If no information about the site is known, an assumption that the site is normally dispersive can be a good preliminary guide since geologic processes tend to create this type of stratigraphy. Knowledge of what velocity values to expect is always preferred though and can give bounds to the anticipated profile. Tables 
on shear wave velocity values based on geologic units, surrounding topography, or nearby surveys can help to give these bounds. An example of one of these tables can be seen in Table 3.1 which shows the Pacific Earthquake Engineering Research Center's (PEER) description of the shear wave velocity characteristics of geologic units in California from Wills and Clahan (2006).

While being observant of the velocity values, the error between the observed/selected dispersion curve and the modeled dispersion curve can be lessened through Geogiga Seismic Pro by selecting "Start" from the "Inversion" drop-down menu or by pressing $>$ in the ribbon. This will run the inversion process again with the initial values being those created from the “Autobuild Initial Model." The additional iterations should reduce the error between the models and produce a new shear wave velocity profile in red. Note that layers can be removed from or added to the model by holding down Ctrl and selecting a layer. 


\begin{tabular}{|c|c|c|c|c|c|c|c|}
\hline $\begin{array}{l}\text { Geologic } \\
\text { Unit }\end{array}$ & Geologic Description & $\begin{array}{l}\text { No. of } \\
\text { Profiles }\end{array}$ & $\begin{array}{c}\text { Mean } \\
V_{s, 30}\end{array}$ & $\begin{array}{l}\text { Std } \\
\text { Dev }\end{array}$ & $\begin{array}{l}V_{s, 30} \text { from } \\
\text { Mean of In }\end{array}$ & $\begin{array}{l}\text { Std } \\
\text { Dev of } \\
\text { In }\end{array}$ & $\begin{array}{l}\text { Mean of } \\
\text { In of } \\
V_{s, 30}\end{array}$ \\
\hline Qi & $\begin{array}{l}\text { Intertidal Mud, including mud around the San } \\
\text { Francisco Bay and similar mud in the } \\
\text { Sacramento/San Joaquin delta and in Humboldt Bay }\end{array}$ & 20 & 160 & 39 & 155 & 0.243 & 5.046 \\
\hline af/qi & $\begin{array}{l}\text { Artificial fill over intertidal mud around San } \\
\text { Francisco Bay }\end{array}$ & 44 & 217 & 94 & 202 & 0.357 & 5.310 \\
\hline Qal, fine & $\begin{array}{l}\text { Quaternary (Holocene) alluvium in areas where it is } \\
\text { known to be predominantly fine }\end{array}$ & 13 & 236 & 55 & 229 & 0.238 & 5.437 \\
\hline Qal, deep & $\begin{array}{l}\text { Quaternary (Holocene) alluvium (Holocene and } \\
\text { Pleistocene) is more than } 30 \text { meters thick; generally } \\
\text { much more in deep basins }\end{array}$ & 161 & 280 & 74 & 271 & 0.250 & 5.604 \\
\hline $\begin{array}{l}\text { Qal, deep } \\
\text { Imperial } \\
\text { Valley }\end{array}$ & $\begin{array}{l}\text { Quaternary (Holocene) alluvium in the Imperial } \\
\text { Valley, except sites in the northern Coachella Valley } \\
\text { adjacent to the mountain front }\end{array}$ & 53 & 209 & 31 & 207 & 0.135 & 5.335 \\
\hline $\begin{array}{l}\text { Qal, deep } \\
\text { LA Basin }\end{array}$ & $\begin{array}{l}\text { Quaternary (Holocene) alluvium in the Los Angeles } \\
\text { basin, except sites adjacent to the mountain fronts }\end{array}$ & 64 & 281 & 85 & 270 & 0.275 & 5.599 \\
\hline Qal, thin & $\begin{array}{l}\text { Quaternary (Holocene) alluvium in narrow valleys, } \\
\text { small basins, and adjacent to the edges of basins } \\
\text { where the alluvium would be expected to be } \\
\text { underlain by contrasting material within } 30 \text { meters }\end{array}$ & 65 & 349 & 89 & 338 & 0.244 & 5.825 \\
\hline $\begin{array}{l}\text { Qal, thin } \\
\text { West LA }\end{array}$ & $\begin{array}{l}\text { Quaternary (Holocene) alluvium in part of west Los } \\
\text { Angeles where the Holocene alluvium is known to } \\
\text { be thin, and is underlain by Pleistocene alluvium }\end{array}$ & 41 & 297 & 45 & 294 & 0.150 & 5.684 \\
\hline $\begin{array}{l}\text { Qal, } \\
\text { coarse }\end{array}$ & $\begin{array}{l}\text { Quaternary (Holocene) alluvium near fronts of high, } \\
\text { steep mountain ranges and in major channels where } \\
\text { the alluvium is expected to be coarse }\end{array}$ & 18 & 354 & 82 & 345 & 0.223 & 5.845 \\
\hline Qoa & Quaternary (Pleistocene) alluvium & 132 & 387 & 142 & 370 & 0.273 & 5.916 \\
\hline Qs & $\begin{array}{l}\text { Quaternary (Pleistocene) sand deposits, such as the } \\
\text { Merritt Sand in the Oakland area }\end{array}$ & 15 & 302 & 46 & 297 & 0.171 & 5.697 \\
\hline QT & $\begin{array}{l}\text { Quaternary to Tertiary (Pleistocene-Pliocene) } \\
\text { alluvial deposits such as the Saugus Formation of } \\
\text { southern California, Paso Robles Formation of } \\
\text { central coast ranges, and the Santa Clara Formation } \\
\text { of the Bay Area }\end{array}$ & 18 & 455 & 150 & 438 & 0.266 & 6.083 \\
\hline Tsh & $\begin{array}{l}\text { Tertiary (mostly Miocene, Oligocene, and Eocene) } \\
\text { shale and siltstone units such as the Repetto, } \\
\text { Fernando, Puente, and Modelo Formations of the } \\
\text { Los Angeles area }\end{array}$ & 55 & 390 & 112 & 376 & 0.272 & 5.930 \\
\hline Tss & $\begin{array}{l}\text { Tertiary (mostly Miocene, Oligocene, and Eocene) } \\
\text { sandstone units such as the Topanga Formation in } \\
\text { the Los Angeles area and the Butano sandstone in } \\
\text { the San Francisco Bay area }\end{array}$ & 24 & 515 & 215 & 477 & 0.386 & 6.169 \\
\hline Tv & $\begin{array}{l}\text { Tertiary volcanic units including the Conejo } \\
\text { Volcanics in the Santa Monica Mountains and the } \\
\text { Leona Rhyolite in the East Bay Hills }\end{array}$ & 3 & 609 & 155 & 597 & 0.240 & 6.392 \\
\hline Kss & $\begin{array}{l}\text { Cretaceous sandstone of the Great Valley Sequence } \\
\text { in the central Coast Ranges }\end{array}$ & 6 & 566 & 199 & 539 & 0.332 & 6.291 \\
\hline Serpentine & $\begin{array}{l}\text { Serpentine, generally considered part of the } \\
\text { Franciscan complex }\end{array}$ & 6 & 653 & 137 & 641 & 0.204 & 6.464 \\
\hline KJf & $\begin{array}{l}\text { Franciscan complex rock, including mélange, shale, } \\
\text { chert, and greenstone }\end{array}$ & 32 & 782 & 359 & 712 & 0.432 & 6.569 \\
\hline xtaline & $\begin{array}{l}\text { Crystalline rocks, including Cretaceous granitic } \\
\text { rocks, Jurassic metamorphic rocks, schist, and } \\
\text { Precambrian gneiss }\end{array}$ & 28 & 748 & 430 & 660 & 0.489 & 6.493 \\
\hline
\end{tabular}

Table 3.1 Shear wave velocity characteristics of geologic units in CA from Wills and Clahan 


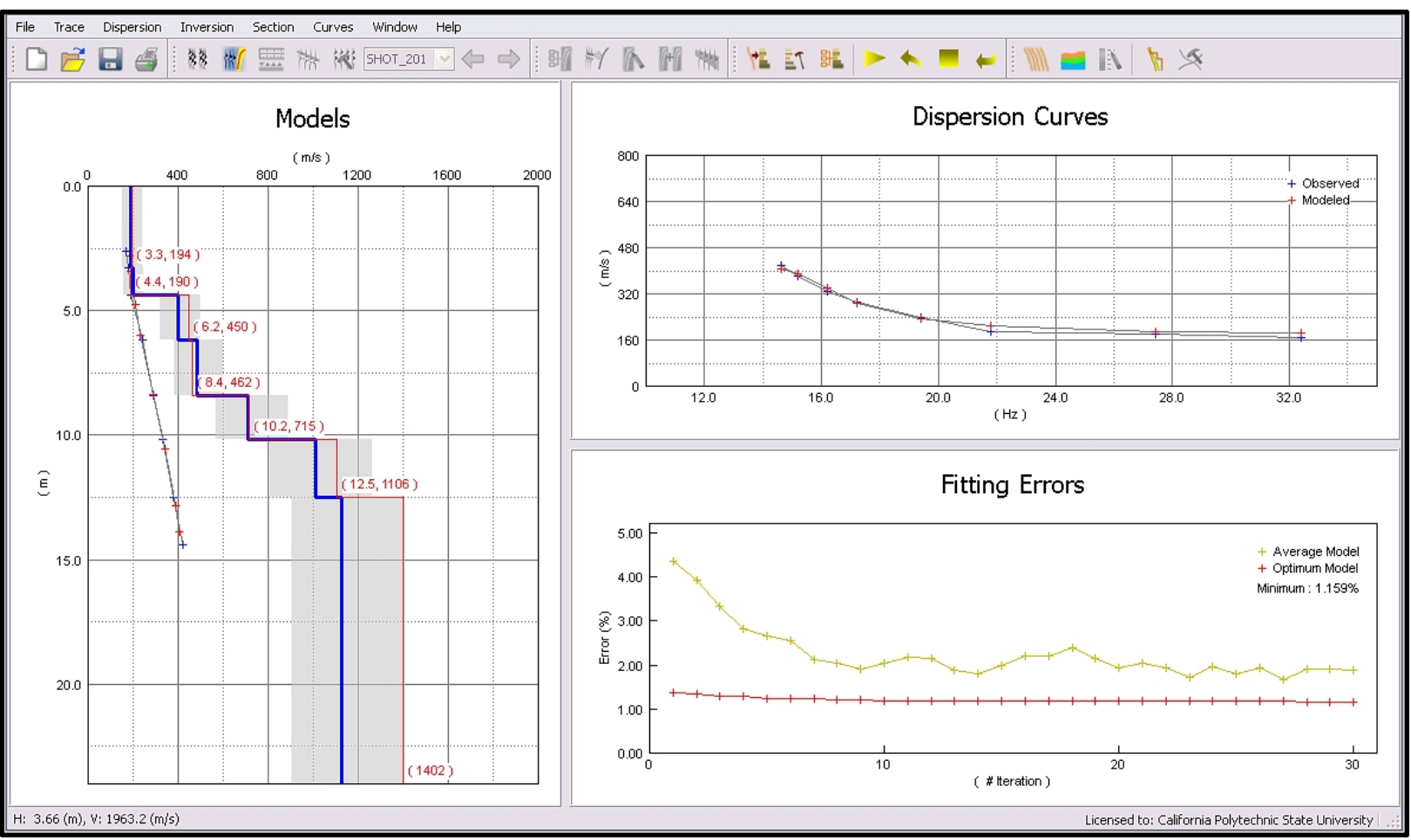

Figure 3.19 The shear wave velocity profile, dispersion curve, and error iteration windows

This process can be repeated by pressing "Reset" in the "Inversion" drop-down menu or by pressing in the ribbon. This changes the initial model to be the one just created (in red), and then pressing "Start" from the "Inversion" drop-down menu will run more iterations. This inversion process should continue to lower the error and make the dispersion curves more similar. However, a representative profile is the goal of this process, not a low error rate, and, although these can be related, too many iterations tend to produce obscure results. This is when expectations of the geology can help decide when the model shows a reasonable profile along with a suitably low error rate. Since it is hard to know when to stop without actually running too many iterations, trial and error is often the most helpful resource. Through this trial and error, a calculation of the uncertainty in the inversion results can help constrain the data (could calculate the median value and iterate to this value). If the profile has gone through too many iterations, 
"Autobuild Initial Model" and then the "Reset" button can be selected in order to start from the beginning.

To keep the shear wave velocity models consistent, this study created profiles using the same procedure for each location. This procedure is site specific and was generated by noting how the velocity profiles changed with several iterations at multiple locations. Three layers were used unless the dispersion curve showed evidence of an inverted layer. Iterations were stopped when the error dropped below $2 \%$ or if the velocity values started to vary significantly from adjacent surveys. If the error after the first round of iterations was below $2 \%$, then one more iteration was complete. Since the recordings for this study overlapped, this procedure was created in an attempt to make neighboring profiles similar to one another, and to keep velocity values reasonable based on the known geology. 


\section{Chapter 4: Results}

\subsection{SPAC Results}

Each survey was taken at 5-meter spacing following the procedures outlined in Chapter 3. The approximate testing locations can be seen in Figure 3.2, and the results of the inversion procedure are summarized in Figure 4.1. Note that the vertical scale on this figure has been exaggerated so that the velocity values can be seen more easily. All of the graphs for the individual surveys can be seen in the Appendix.

Three depths were analyzed based on the amount of available data. The average sample depth was from 3.2 to 17.3 meters from the ground surface, so depths of 5, 10, and 15 meters from the ground surface were analyzed. A semi-variogram was also created for data at 20 meters depth, but the small amount of data at this depth made the plot too erratic. Note that, since there is an elevation change at the site, all depth values in this study are in reference to the ground surface in the valley (not on the hill).

This study used the time weighted average velocity since this is what is used in practice (and required by the NEHRP site classification guidelines). This allows a column of geologic material to be analyzed instead of point values. It is estimated by dividing the depth by the amount of time it took a wave to propagate through each layer within that depth. The equation can be written as;

$$
\bar{V}=\frac{d_{T}}{\sum_{i=1}^{n} \frac{d_{i}}{V_{i}}}
$$

where $\bar{V}$ is the time weighted average velocity of the column, $d_{T}$ is the total depth of interest, $d_{i}$ is the thickness of each layer within $d_{T}, V_{i}$ is the velocity of each layer, and $n$ 


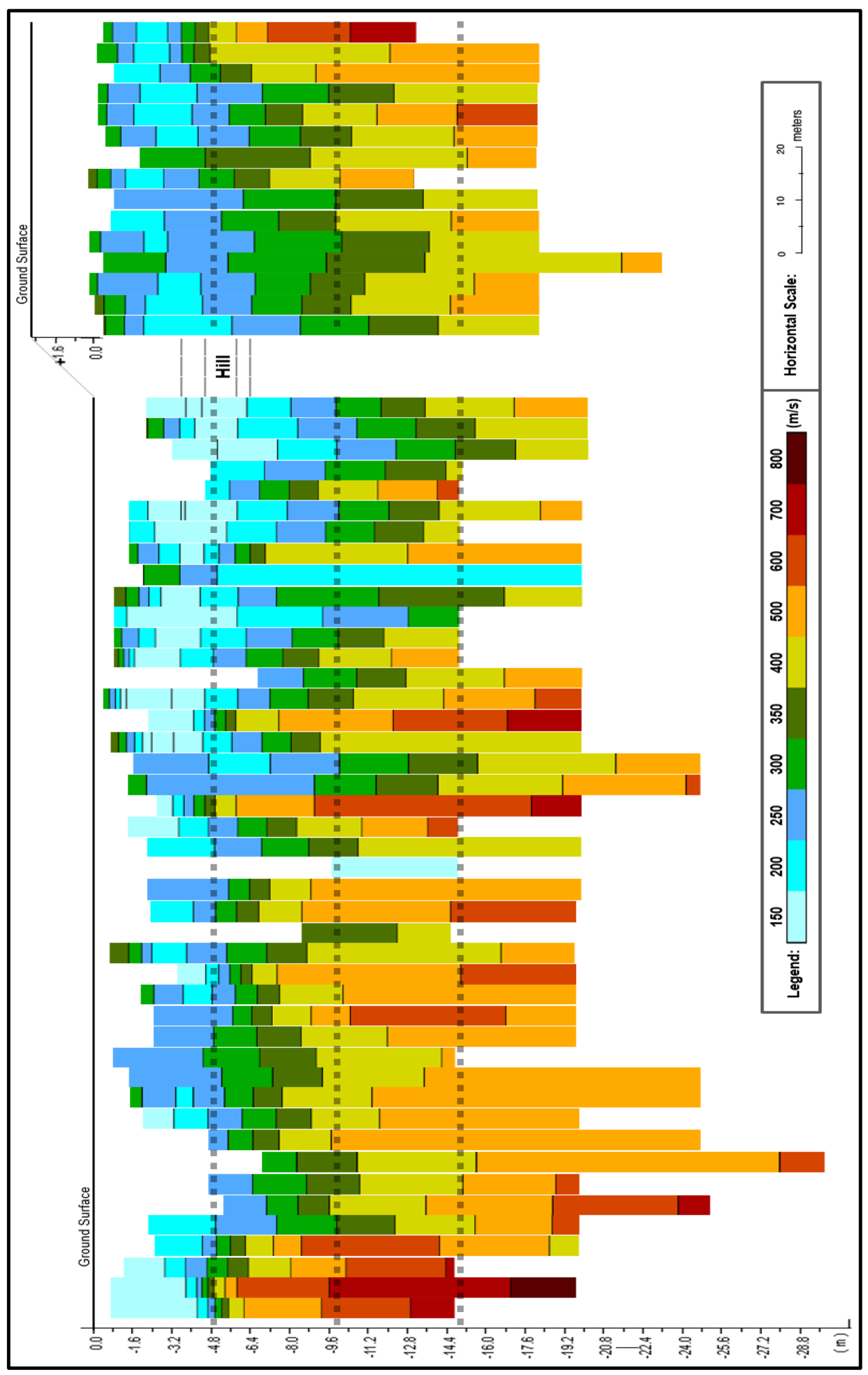

Figure 4.1 Shear wave velocity profile for each survey with dotted lines at analyzed depths 


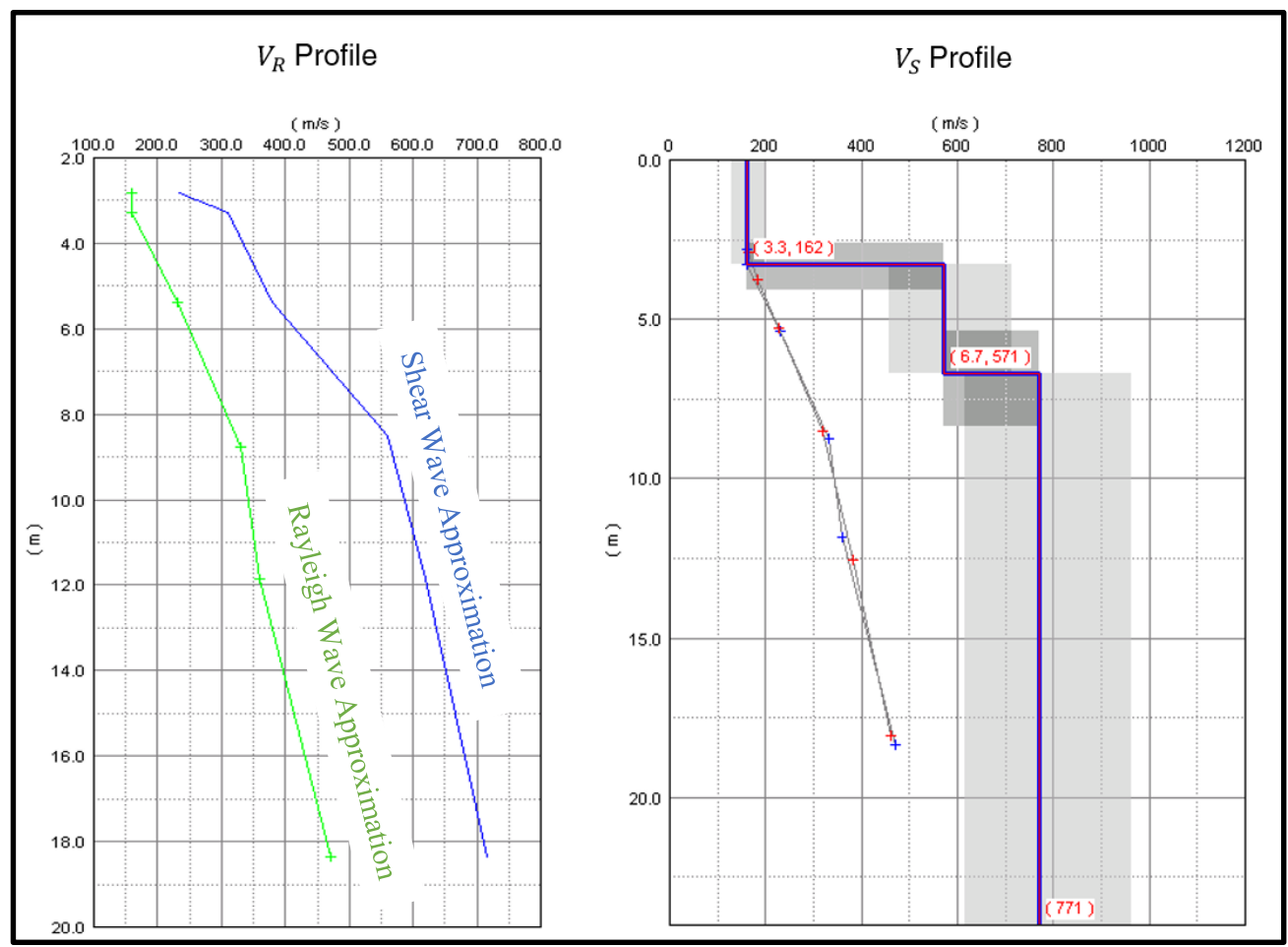

Figure 4.2 Example of Rayleigh wave and shear wave velocity profiles from Geogiga (2012) represents the total number of layers. This equation is easy to calculate when using shear wave velocity profiles because of their "stepped" nature, but the Rayleigh wave velocity profile is estimated via the dispersion curve (from Rix and Leipski, 1991, half-wavelength approximation) which does not have constant, layered velocity values. A comparison can be seen if Figure 4.2. In order to analyze the Rayleigh wave velocity profiles, the same concept was applied. Each segment was treated as a layer and average velocity values were used for each layer. This weights the velocity based on the average time it took waves to propagate through each segment. The results for both the Rayleigh wave and shear wave velocity values can be seen in the Appendix.

The time weighted average Rayleigh wave velocities $\left(\bar{V}_{R}\right)$ at 5 meters, 10 meters, and 15 meters depth can be seen in Figure 4.3. Similarly, the time weighted average shear wave velocities $\left(\bar{V}_{S}\right)$ at these depths can be seen in Figure 4.4. The offsets in the figure are related to 
the reference stake (see Figure 3.2) where offsets north of this stake are negative and south of this stake are positive. The gap in the data corresponds to the 3-array break due to the hill seen in Figure 3.2. The higher velocity values past the hill are assumed to be due to the additional overburden stress that compressed the material below.

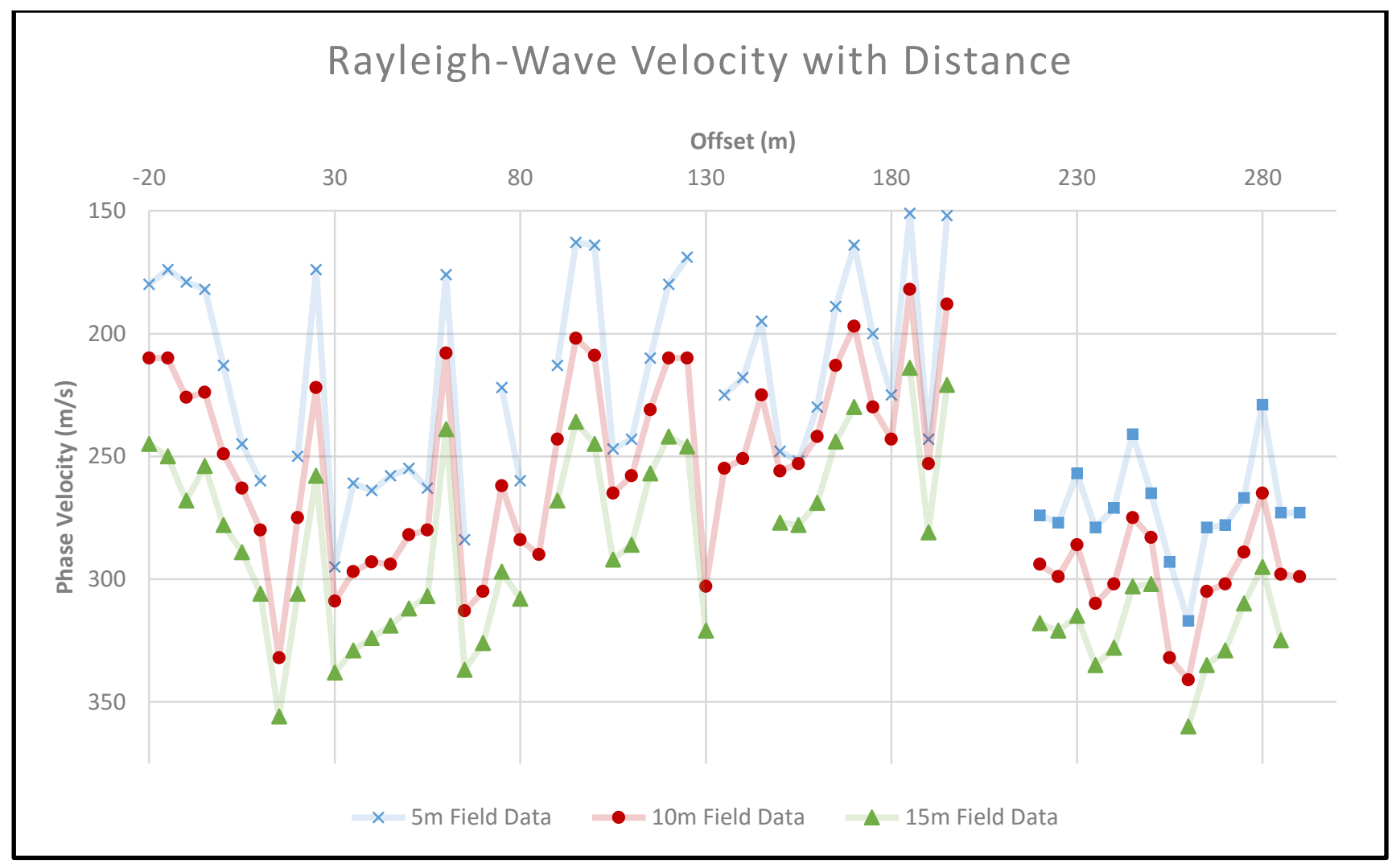

Figure 4.3 Time-averaged Rayleigh wave velocity across the site 


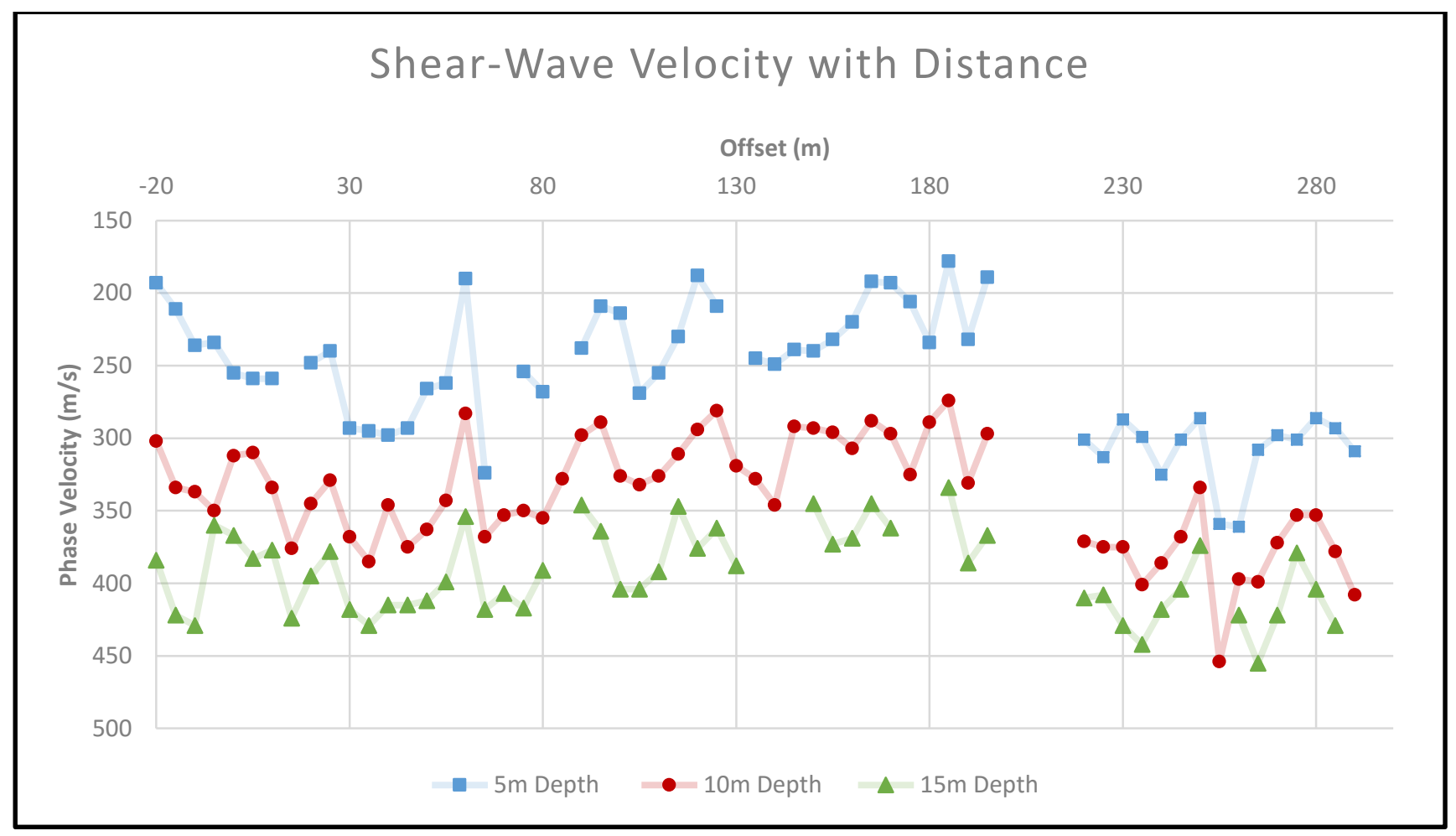

Figure 4.4 Time-averaged shear wave velocity across the site

\subsection{Empirical Semi-Variograms and Covariance Functions}

The semi-variogram and covariance function using all of the $\bar{V}_{R}$ data can be seen in Figure 4.5 with the corresponding number of data pairs used for each point. Note that the numerical subscripts refer to the analyzed depth. The $\bar{V}_{R, 10}$ plots are the most constrained which appears to be from the additional data and the geologic similarities at this depth. The covariance function, however, flattens at negative values, and this behavior is indicative of a trend/nonstationarity in the data (Oliver and Webster, 2010). In other words, the mean value is not constant throughout the site.

The seemingly obvious source of the apparent trend is from the distinct increase in velocity values after the hill. In attempts to rid the data of this trend, the "hill" data was removed from the set and re-analyzed; these results can be seen in Figure 4.8. Another option for removing this apparent trend is to fit a trendline to the data and then create a semi-variogram 
from the residuals (Oliver and Webster, 2015). This tries to model the changing mean value so that the spatial statistics are calculated with a pseudo-constant mean value. A polynomial function and a piecewise linear function were used as the trendlines because these fit closest to the data. The largest $R^{2}$ value (while still being parsimonious) was from a fourth-order polynomial function and the piecewise-linear function was split into sections before the hill and after the hill. The trendlines can be seen in Figure 4.6 and Figure 4.7, respectively, and the geostatistical results can be seen in Figure 4.9 and Figure 4.10, respectively.

When looking at the piecewise-linear plot, the data could also be modeled as a simple linear function without the "hill" data (i.e. the first portion of the piecewise-linear function). This models the data in case there was a trend within the valley. These results can be seen in Figure 4.11. 


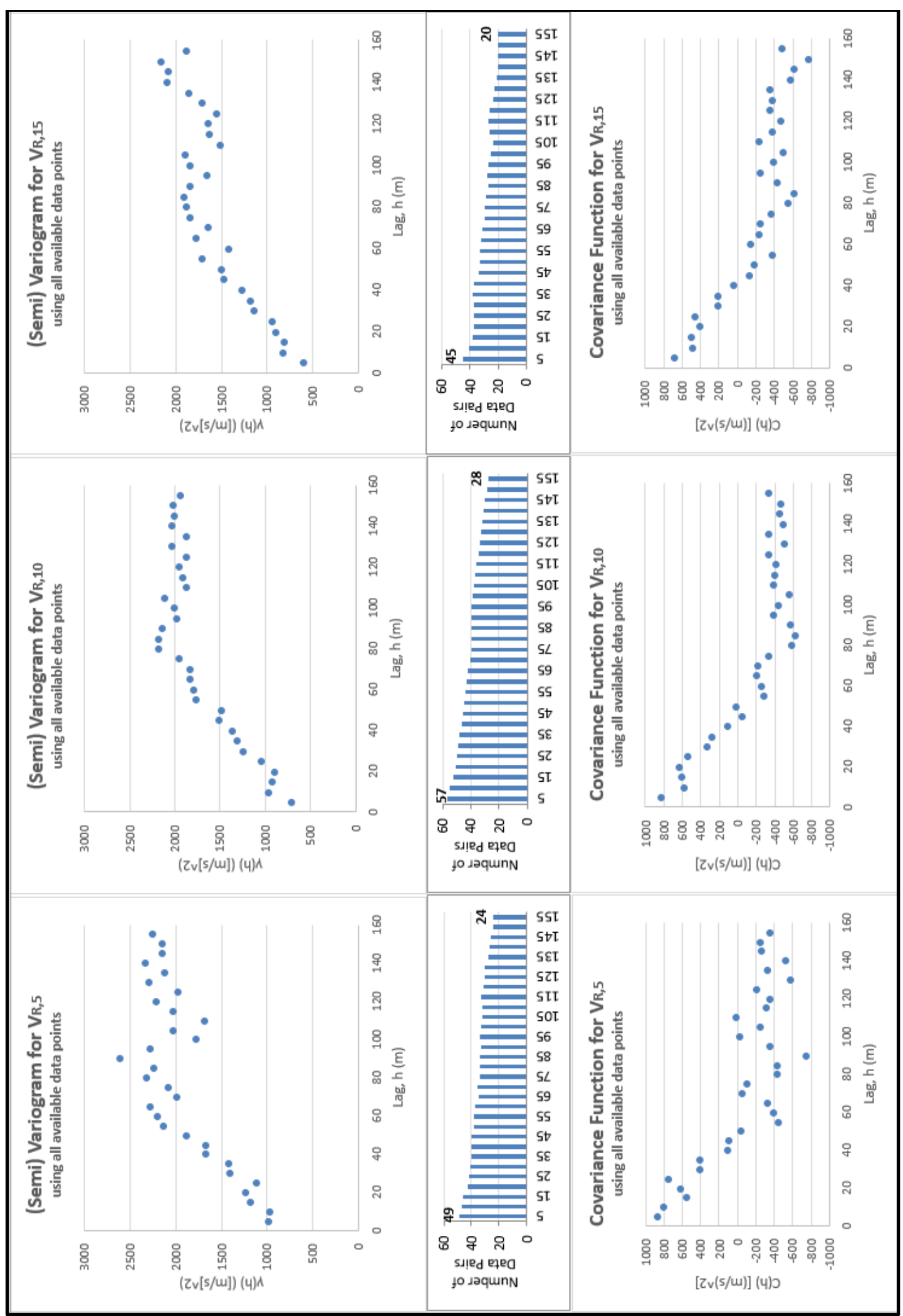

Figure 4.5 Semi-variogram and covariance function for time weighted average Rayleigh wave velocity using all available data points 


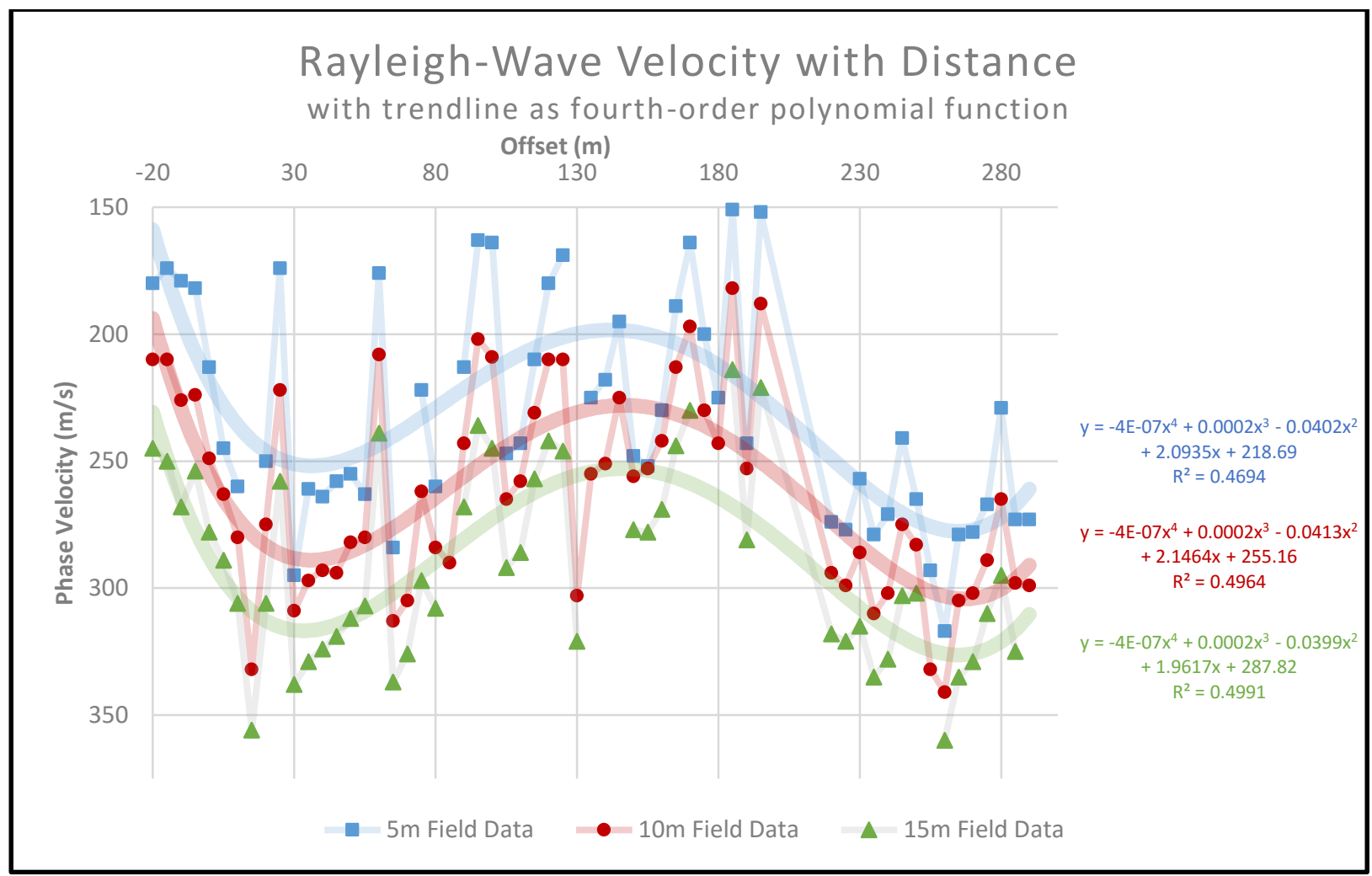

Figure 4.6 Time weighted average Rayleigh wave velocity fit with fourth-order polynomial functions

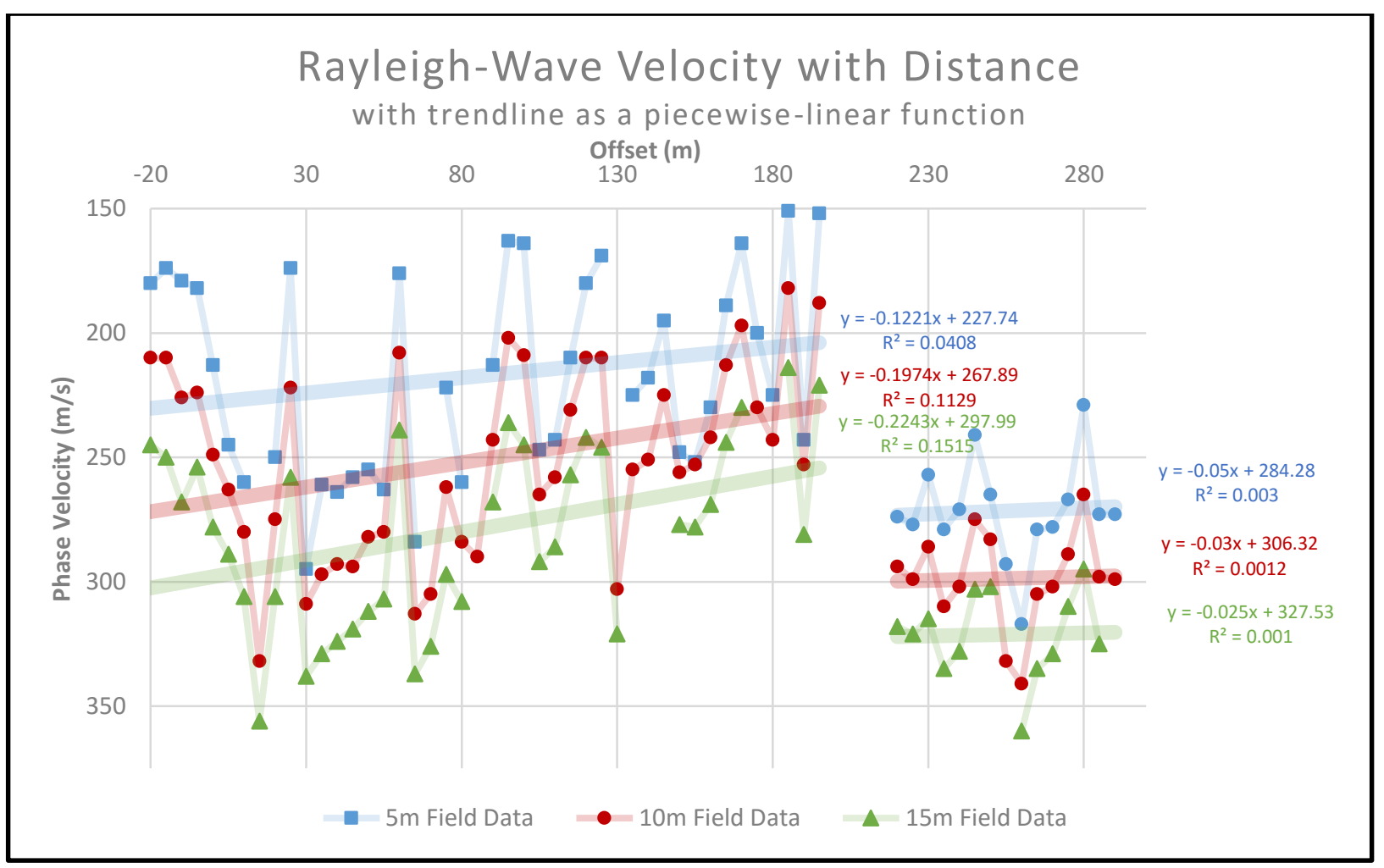

Figure 4.7 Time weighted average Rayleigh wave velocity fit with piecewise-linear functions 


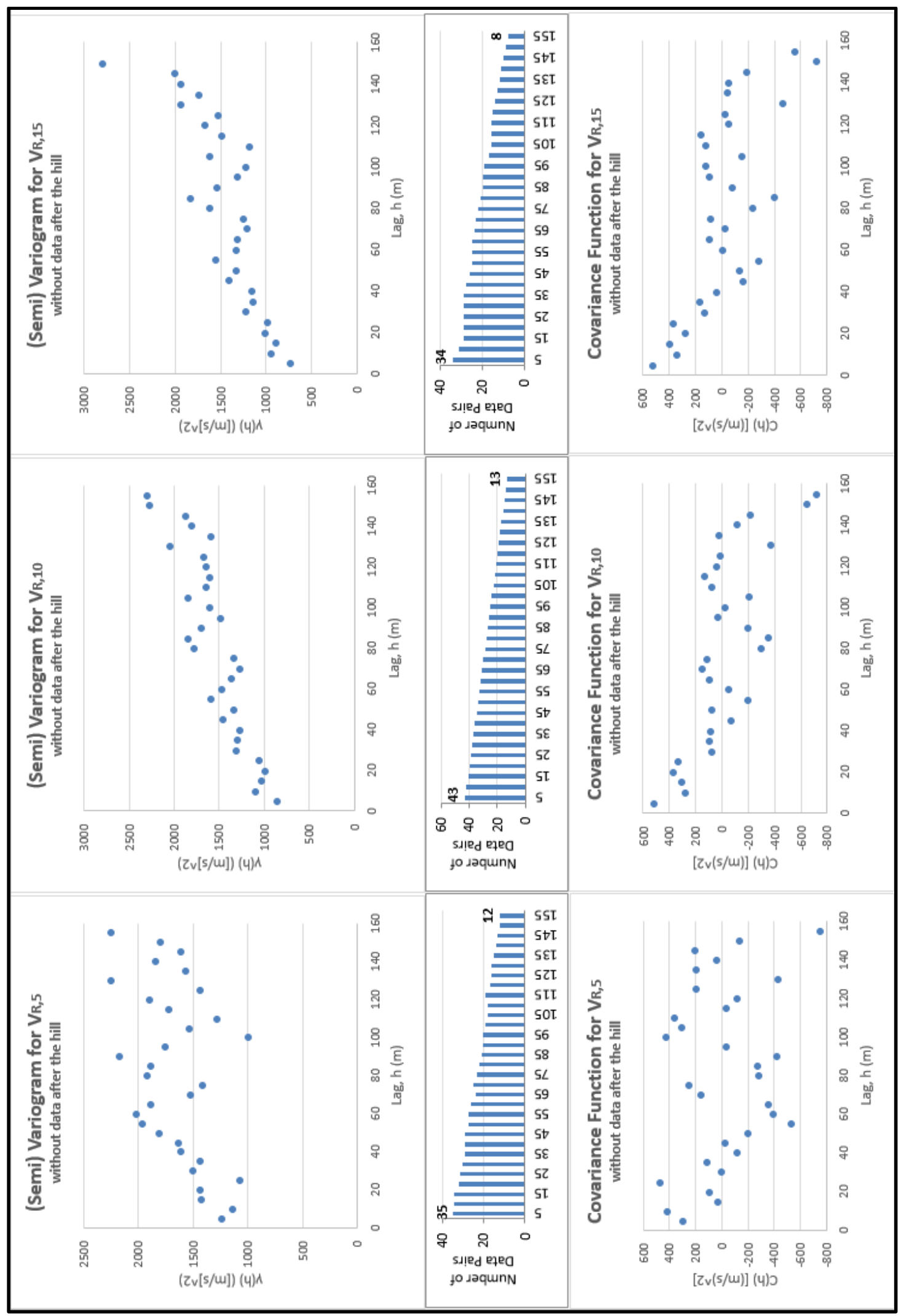

Figure 4.8 Semi-variogram and covariance function for time weighted average Rayleigh wave velocity not including data from after the hill 


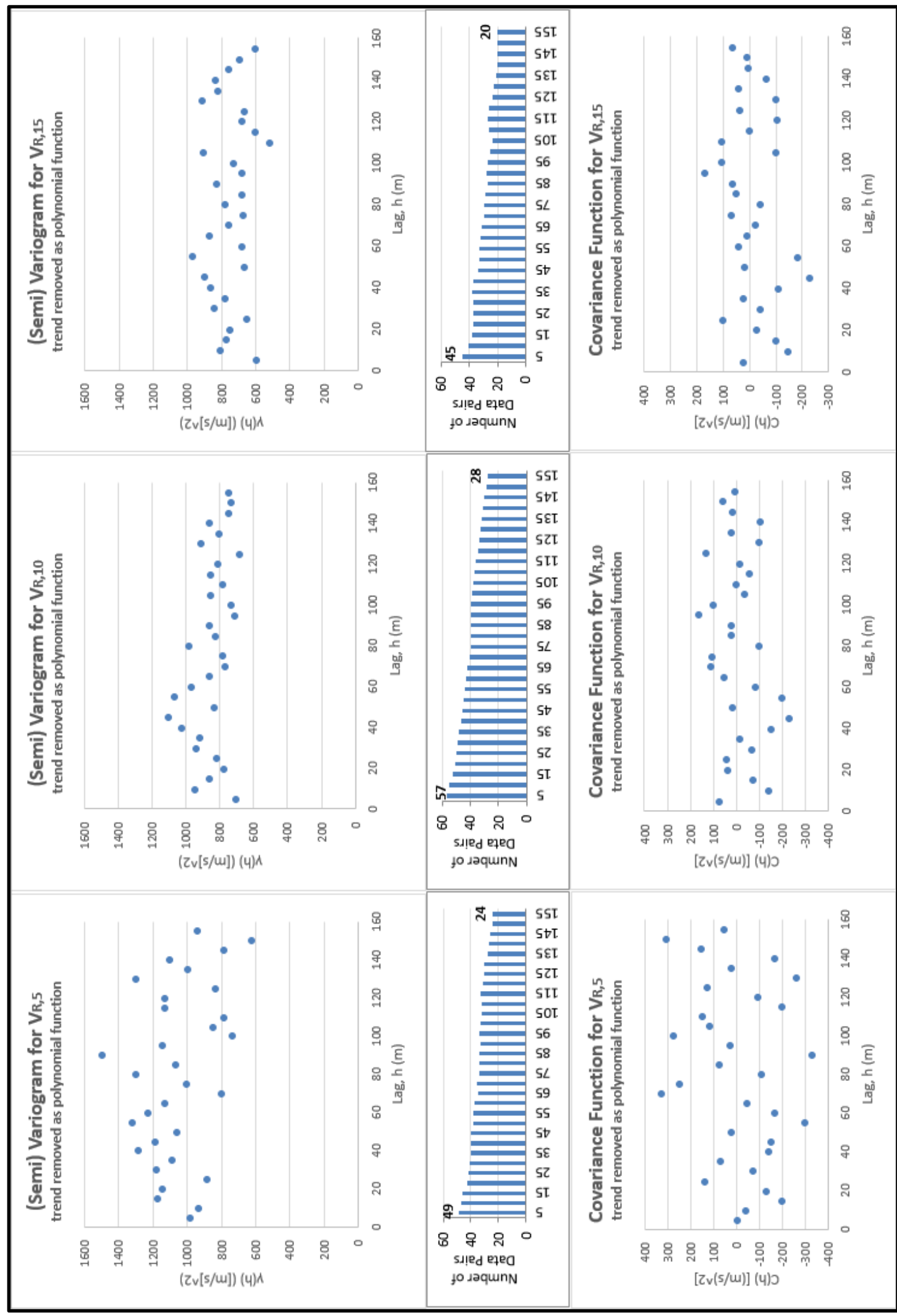

Figure 4.9 Semi-variogram and covariance function for time weighted average Rayleigh wave velocity with trend removed as a fourth-order polynomial function 


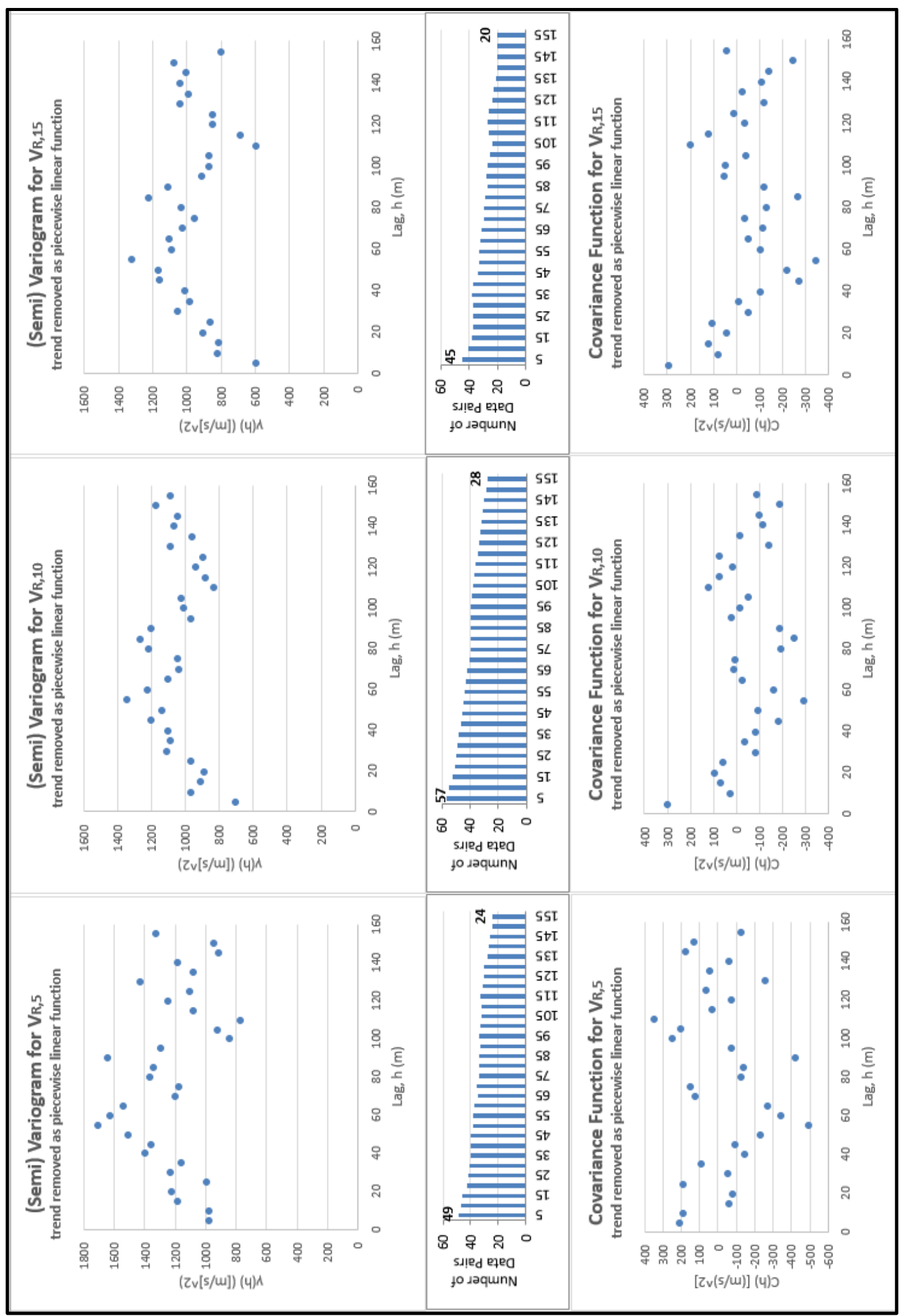

Figure 4.10 Semi-variogram and covariance function for time weighted average Rayleigh wave velocity with trend removed as a piecewise-linear function 


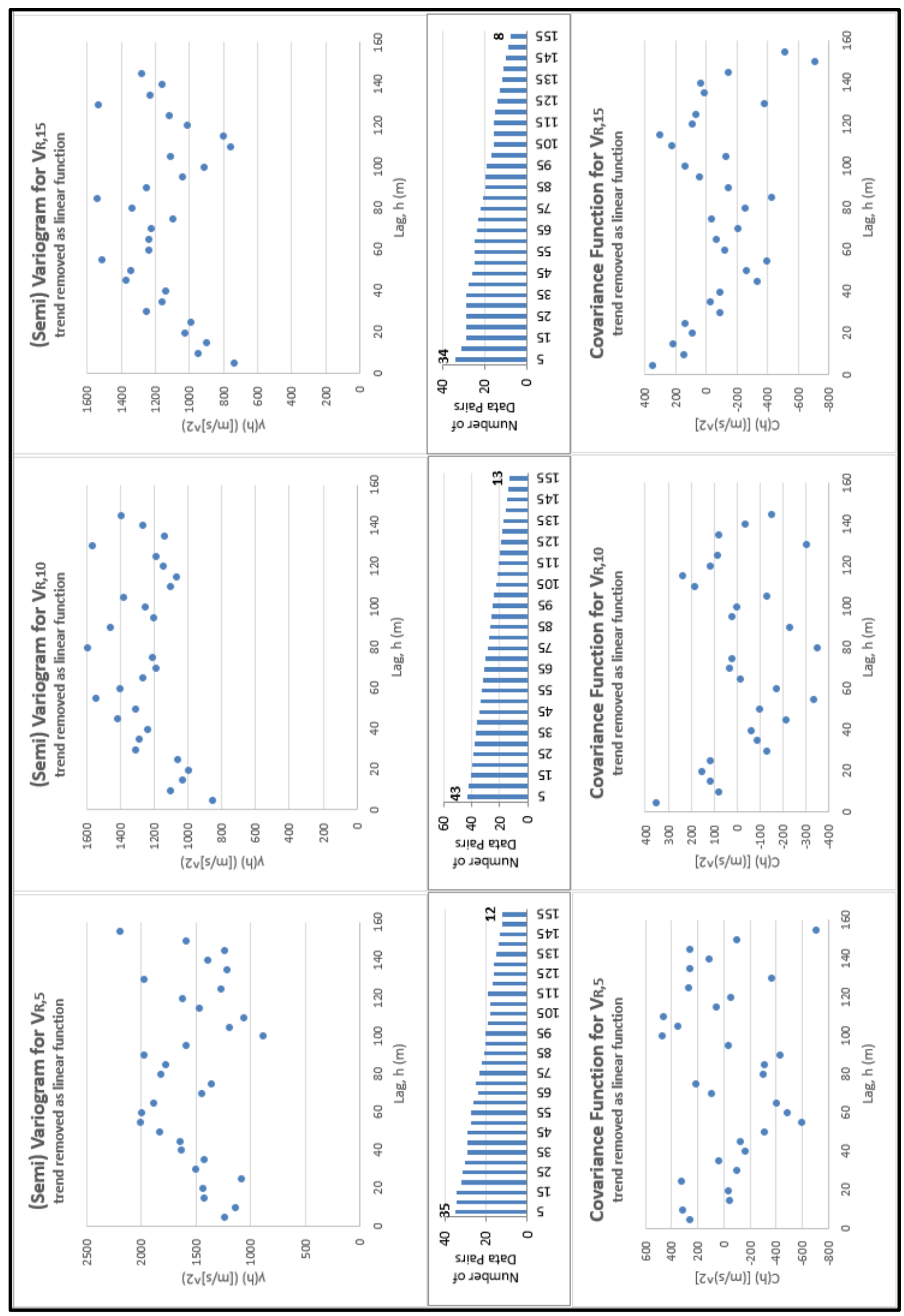

Figure 4.11 Semi-variogram and covariance function for time weighted average Rayleigh wave velocity with trend removed as a linear function (without data after hill) 


\subsection{Modeling the Semi-Variograms}

Notice that the plots become more erratic as the amount of data pairs decreases - this is typical (Oliver and Webster, 2015). Also note that the semi-variograms using the residuals do not appear to constrain the data any better. This can be seen more easily through modeling the data. All of these semi-variograms were modeled with spherical, exponential, and Gaussian models. These three models were chosen because they are the common transition models (models including a sill) and a sill is expected for alluvial material (e.g. Iqbal et al., 2005, and Facciorusso et al., 2010). Oliver and Webster (2015) recommend choosing a semi-variogram model using the coefficient of determination $\left(R^{2}\right)$ and any knowledge of the geologic behavior. The $R^{2}$ values for each semi-variogram model and the different remedies for the apparent trend can be seen in Table 4.1. Additionally, the semi-variograms, covariance functions, and correlograms for each of these options can be seen in the Appendix.

\begin{tabular}{|c|c|c|c|c|c|c|c|}
\hline \multirow[b]{2}{*}{ Data Used } & \multirow[b]{2}{*}{ Model } & \multicolumn{3}{|c|}{$\begin{array}{c}\text { Rayleigh wave } \\
\text { Velocity Data }\end{array}$} & \multicolumn{3}{|c|}{$\begin{array}{c}\text { Shear wave } \\
\text { Velocity Data }\end{array}$} \\
\hline & & $\begin{array}{l}5 \mathrm{~m} \\
\text { Depth }\end{array}$ & $\begin{array}{l}10 \mathrm{~m} \\
\text { Depth }\end{array}$ & $\begin{array}{l}\text { 15m } \\
\text { Depth }\end{array}$ & $\begin{array}{l}5 \mathrm{~m} \\
\text { Depth }\end{array}$ & $\begin{array}{l}10 \mathrm{~m} \\
\text { Depth }\end{array}$ & $\begin{array}{l}\text { 15m } \\
\text { Depth }\end{array}$ \\
\hline \multirow{3}{*}{ 1. All Available Data } & Spherical & $83 \%$ & $95 \%$ & $86 \%$ & $94 \%$ & $91 \%$ & $72 \%$ \\
\hline & Exponential & $79 \%$ & $91 \%$ & $86 \%$ & $88 \%$ & $87 \%$ & $70 \%$ \\
\hline & Gaussian & $84 \%$ & $95 \%$ & $86 \%$ & $94 \%$ & $92 \%$ & $73 \%$ \\
\hline \multirow{3}{*}{$\begin{array}{l}\text { 2. Data after Hill } \\
\text { Removed }\end{array}$} & Spherical & $36 \%$ & $76 \%$ & $68 \%$ & $68 \%$ & $78 \%$ & $50 \%$ \\
\hline & Exponential & $31 \%$ & $76 \%$ & $68 \%$ & $65 \%$ & $76 \%$ & $49 \%$ \\
\hline & Gaussian & $35 \%$ & $74 \%$ & $68 \%$ & $69 \%$ & $79 \%$ & $54 \%$ \\
\hline \multirow{3}{*}{$\begin{array}{l}\text { 3. Trend Removed as } \\
\text { Linear Function } \\
\text { without Data after Hill }\end{array}$} & Spherical & $23 \%$ & $57 \%$ & $48 \%$ & $34 \%$ & $27 \%$ & $3 \%$ \\
\hline & Exponential & $19 \%$ & $55 \%$ & $45 \%$ & $30 \%$ & $24 \%$ & $3 \%$ \\
\hline & Gaussian & $22 \%$ & $57 \%$ & $48 \%$ & $33 \%$ & $27 \%$ & $3 \%$ \\
\hline \multirow{3}{*}{$\begin{array}{l}\text { 4. Trend Removed as } \\
\text { Piecewise Linear } \\
\text { Function }\end{array}$} & Spherical & $0.8 \%$ & $24 \%$ & $21 \%$ & $39 \%$ & $8 \%$ & $0.4 \%$ \\
\hline & Exponential & $8 \%$ & $24 \%$ & $21 \%$ & $42 \%$ & $8 \%$ & $0.6 \%$ \\
\hline & Gaussian & $0.5 \%$ & $23 \%$ & $20 \%$ & $41 \%$ & $7 \%$ & $0.8 \%$ \\
\hline \multirow{3}{*}{$\begin{array}{l}\text { 5. Trend Removed as } \\
\text { 4th Order Polynomial }\end{array}$} & Spherical & $7 \%$ & $15 \%$ & $0.3 \%$ & $16 \%$ & $7 \%$ & $4 \%$ \\
\hline & Exponential & $4 \%$ & $14 \%$ & $0.3 \%$ & $15 \%$ & $7 \%$ & $4 \%$ \\
\hline & Gaussian & $6 \%$ & $6 \%$ & $0.6 \%$ & $2 \%$ & $7 \%$ & $4 \%$ \\
\hline
\end{tabular}

Table 4.1 Coefficient of determination values for each data type and semi-variogram model 


\begin{tabular}{|c|c|c|c|c|c|c|c|}
\hline & & \multicolumn{3}{|c|}{ Rayleigh wave Velocity Data } & \multicolumn{3}{|c|}{ Shear wave Velocity Data } \\
\hline \multicolumn{2}{|c|}{ Data Used } & $5 \mathrm{~m}$ Depth & 10m Depth & $15 \mathrm{~m}$ Depth & $5 \mathrm{~m}$ Depth & $10 \mathrm{~m}$ Depth & 15m Depth \\
\hline \multirow{2}{*}{ 1. All Available Data } & \multirow{2}{*}{$\begin{array}{l}\text { Spherical } \\
\text { Gaussian }\end{array}$} & 81 & 92 & 89 & 97 & 131 & 142 \\
\hline & & 50 & 75 & 60 & 70 & 100 & 100 \\
\hline \multirow{2}{*}{$\begin{array}{l}\text { 2. Data after Hill } \\
\text { Removed }\end{array}$} & \multirow{2}{*}{$\begin{array}{l}\text { Spherical } \\
\text { Gaussian }\end{array}$} & 63 & 129 & 87 & 87 & 270 & 399 \\
\hline & & 50 & 90 & 50 & 60 & - & - \\
\hline \multirow{2}{*}{$\begin{array}{l}\text { 3. Trend Removed as } \\
\text { Linear Function } \\
\text { without Data after Hill }\end{array}$} & Spherical & 57 & 58 & 48 & 60 & 59 & 1668 \\
\hline & Gaussian & 30 & 45 & 30 & 40 & 55 & - \\
\hline \multirow{2}{*}{$\begin{array}{l}\text { 4. Trend Removed as } \\
\text { Piecewise Linear } \\
\text { Function }\end{array}$} & \multirow{2}{*}{$\begin{array}{l}\text { Spherical } \\
\text { Gaussian }\end{array}$} & - & 38 & 34 & 170 & 83 & - \\
\hline & & - & 25 & 25 & 55 & 50 & - \\
\hline \multirow{2}{*}{$\begin{array}{l}\text { 5. Trend Removed as } \\
\text { 4th Order Polynomial }\end{array}$} & \multirow{2}{*}{$\begin{array}{l}\text { Spherical } \\
\text { Gaussian }\end{array}$} & - & - & - & - & - & - \\
\hline & & - & - & - & - & - & - \\
\hline
\end{tabular}

Table 4.2 Semi-variogram ranges from the spherical and Gaussian models for each data type

In Table 4.1, notice that the exponential model gives a worse fit than the spherical or Gaussian models in almost all the cases, whereas the spherical and Gaussian models are nearly identical in terms of $R^{2}$ values. Because of this, the ranges (distance to the sill) were found for the spherical and Gaussian models only. These ranges can be seen in Table 4.2. The range for the spherical models was found using open-source, MATLAB code (Schwanghart, 2010), but this code could not accurately identify the range for Gaussian models. Because of this, the Gaussian models' ranges were estimated manually by taking $95 \%$ of the sill value (the definition of the range for models that reach a sill asymptotically).

The results show that all of the Gaussian ranges are shorter than their spherical counterparts. This is most likely due to the fact that the Gaussian models approach the sill at similar lag distances as the spherical models. While spherical models define their range by when 
the model reaches the sill, the Gaussian model's range is defined by the $95 \%$ of that value and so it will be inherently lower. An example of this can be seen in Figure 4.12.
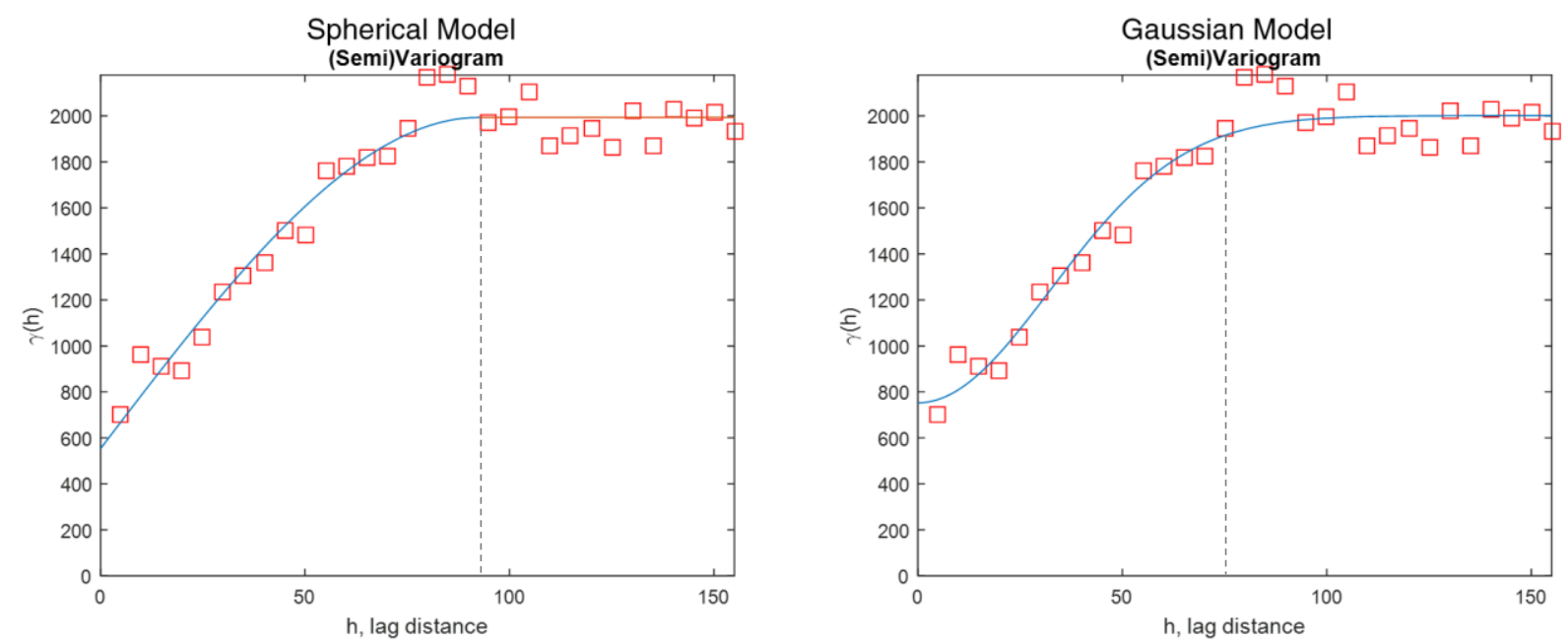

Figure 4.12 Semi-variogram from Rayleigh wave data at $10 \mathrm{~m}$ depth using all available data and being modeled with a spherical model (left) and a Gaussian model (right)

Note that values that are not bolded in Table 4.1 and Table 4.2 have ranges outside the boundaries of the data set. This implies that a transitional model is not appropriate; a linear model would better represent the data in these cases. However, a linear model is not expected for this data set, has no range, and could be indicative of another trend (Oliver and Webster, 2015). Although, the data in these situations appear to be so erratic that a line is the only model that sufficiently lowers the $R^{2}$ value. As a result, these unbolded values should not be considered reliable.

To help give an idea of the shape of these semi-variograms and how well they fit the model, an overview (modeled with spherical models) can be seen in Figure 4.13 where the row numbers correspond to the row numbers in Table 4.1 and Table 4.2. These figures are meant to give a broad sense of how the semi-variograms vary, but, for further inspection, these figures can be seen in detail in the Appendix. 

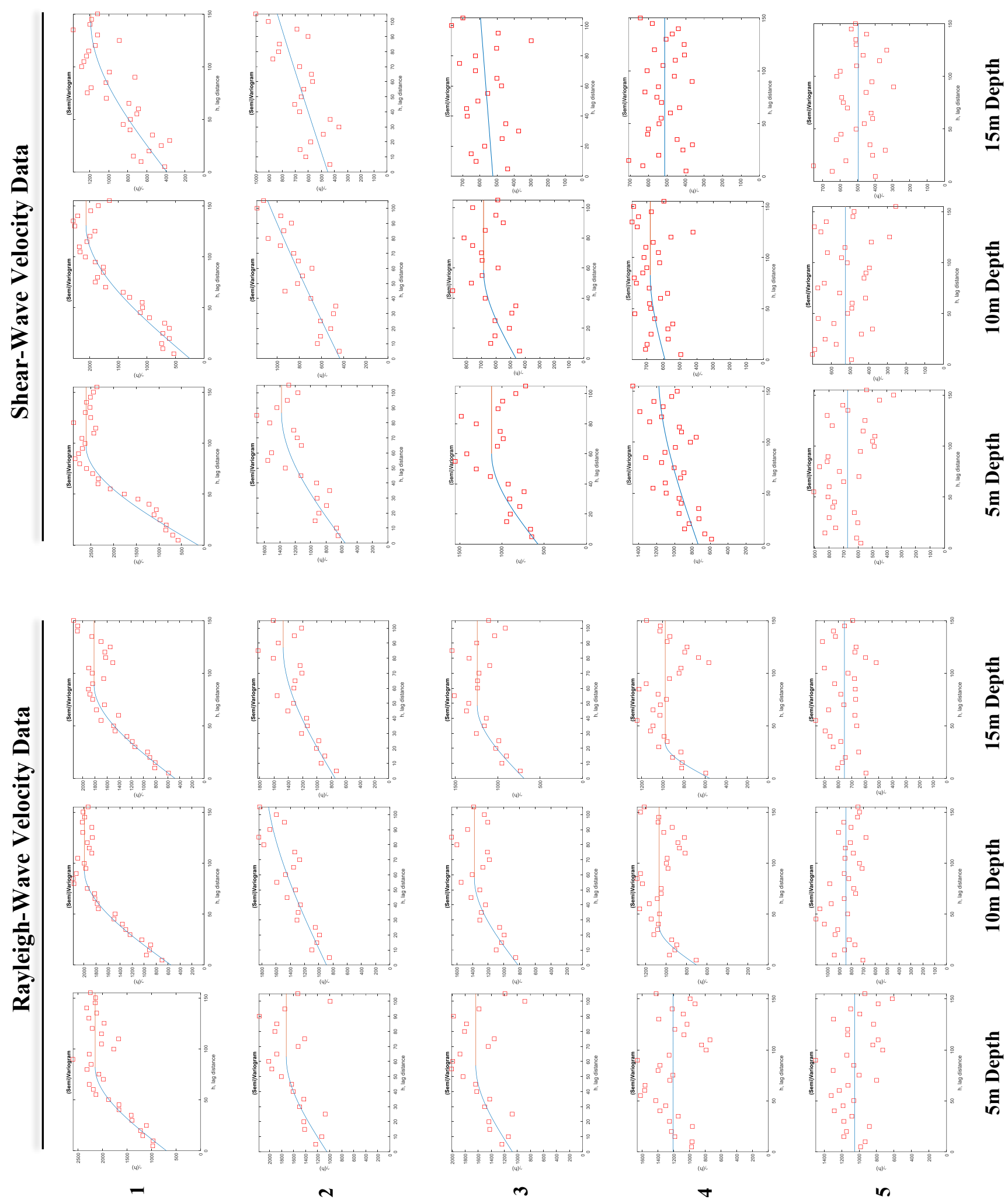

Figure 4.13 Overview of semi-variogram plots fitted with spherical models 


\subsection{Estimating the Ranges}

Given all of this, an applicable range value should be chosen based on all of these factors - the $R^{2}$ values, the semi-variogram shape, and the spread of the range values. The only data whose models explain over half of the variation $\left(R^{2}>50 \%\right)$ are those with all the data included, those with the "hill" data removed, and those with the trend removed as a linear function after the "hill" data was removed. A summary of these range values that have $R^{2}$ values greater than $50 \%$ and whose sills are visible within the data can be seen in Table 4.3.

\begin{tabular}{|c|c|c|c|c|c|c|c|}
\hline & & \multicolumn{3}{|c|}{ Rayleigh wave Velocity Data } & \multicolumn{3}{|c|}{ Shear wave Velocity Data } \\
\hline \multicolumn{2}{|c|}{ Data Used } & $5 \mathrm{~m}$ Depth & 10m Depth & $15 \mathrm{~m}$ Depth & $5 \mathrm{~m}$ Depth & 10m Depth & 15m Depth \\
\hline \multirow{2}{*}{ All Available Data } & Spherical & $81(83 \%)$ & $92(95 \%)$ & $89(86 \%)$ & $97(94 \%)$ & $131(91 \%)$ & $142(72 \%)$ \\
\hline & Gaussian & $50(84 \%)$ & $75(95 \%)$ & 60 (86\%) & 70 (94\%) & 100 (92\%) & $100(73 \%)$ \\
\hline \multirow{2}{*}{$\begin{array}{l}\text { Data after Hill } \\
\text { Removed }\end{array}$} & Spherical & & & $87(68 \%)$ & $87(68 \%)$ & & \\
\hline & Gaussian & & & $50(68 \%)$ & $60(69 \%)$ & & \\
\hline \multirow{2}{*}{$\begin{array}{l}\text { Trend Removed as } \\
\text { Linear Function } \\
\text { without Data after } \\
\text { Hill }\end{array}$} & Spherical & & $58(57 \%)$ & & & & \\
\hline & Gaussian & & $45(57 \%)$ & & & & \\
\hline
\end{tabular}

Table 4.3 Summary of range values whose model has $R^{2}$ values greater than $50 \%$ and whose sill is visible within the data

It is hard to trust the values from the last two data portions because none of the adjacent depths are well modeled and the conversion to shear wave velocity is also not well modeled (or the value was estimated from poorly-modeled Rayleigh wave velocity). And, in regards to removing the apparent trend, these two data portions also give negative covariance functions, so they prove to be no more useful than the initial model. Because of this, the only mathmatically reliable data is that which includes all the available data; the $R^{2}$ values are high for both Rayleigh and shear wave velocity, the semi-variograms are well constrained, and all depths appear to be well modeled. The problem remains that second-order stationarity is violated (i.e. 
that there is not a constant mean value across the site). This indicates that the covariance functions and correlograms are not applicable, however Matheron's intrinsic hypothesis (see Section 2.2.3 Spatial Continuity Models) can be used which allows the semi-variogram values to be applicable even though the covariance function and correlogram are not (Schekhar, 2008).

Using all the data, the ranges are similar at 10 and 15 meter depths but smaller at 5 meters depth. This seems justified since the near-surface material tend to have more differences than deeper material (from events like seasonal influences or utility installations or differing water tables). Assuming that the range values for 10 and 15 meters depth are similar enough that averaged values accurately represent these depths, the results can be seen in Table 4.4.

\begin{tabular}{|c|c|c|c|c|c|}
\hline & & Rayleis & Velocity Data & Shear & Telocity Data \\
\hline Data Used & Model & $5 \mathrm{~m}$ Depth & $\begin{array}{l}10 \mathrm{~m} \text { and } \\
15 \mathrm{~m} \text { Depth }\end{array}$ & $5 \mathrm{~m}$ Depth & $\begin{array}{l}10 \mathrm{~m} \text { and } \\
15 \mathrm{~m} \text { Depth }\end{array}$ \\
\hline \multirow{2}{*}{ All Available Data } & \multirow{2}{*}{$\begin{array}{l}\text { Spherical } \\
\text { Gaussian }\end{array}$} & 81 & 90.5 & 97 & 136.5 \\
\hline & & 50 & 67.5 & 70 & 100 \\
\hline
\end{tabular}

Table 4.4 Range values using all available data and averaging the values from 10 and $15 \mathrm{~m}$ depth Since the $R^{2}$ values are nearly identical between the spherical and Gaussian models, the smaller ranges of the Gaussian models will be used for conservatism. For Rayleigh waves, this gives a correlation range of 50 meters for a 5-meter-deep column of alluvial soil and of approximately 65 meters for a 10- to 15 -meter-deep column of alluvial soil. For shear waves, this gives a correlation range of 70 meters for a 5-meter-deep column of alluvial soil and of 100 meters for a 10- to 15-meter-deep column of alluvial soil.

Boore (2004) gives a correlation that estimates the time weighted average shear wave velocity at 30 meters depth $\left(\bar{V}_{s, 30}\right)$ based on shallower velocity values. This correlation can be seen on the next page; 


$$
\ln \left(\bar{V}_{s, 30}\right)=a+b \ln \left(\bar{V}_{s, d}\right)
$$

where $a$ and $b$ are empirical constants and $\bar{V}_{s, d}$ is the time weighted average shear wave velocity at depth, $d$. Since the values at 10-meters depth were the most constrained/had the most amount of data pairs, this data was used to estimate $\bar{V}_{s, 30}$ using Boore's correlation. The empirical semivariogram and the semi-variogram modeled with a Gaussian model can be seen in Figure 4.14. The additional graphs for this depth can be seen in the Appendix. The range for this estimate is 90 meters.

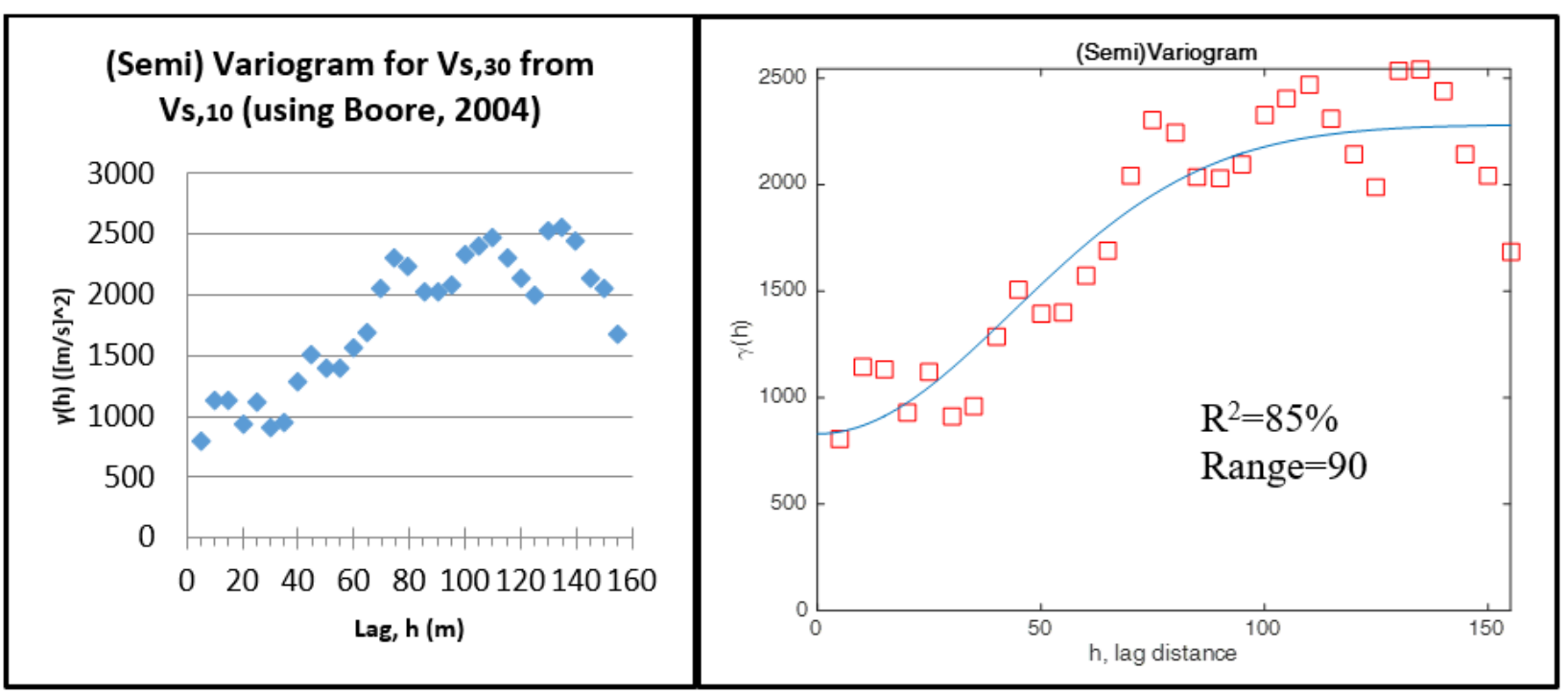

Figure 4.14 Semi-variogram at 30 meters depth from Boore's estimate using velocity values at 10 meters depth; Empirical semi-variogram values (left) and semi-variogram with a Gaussian model (right)

\subsection{Applicability and Limitations}

\subsubsection{Applying Range Values to Other Sites}

The range values are designed to fit the Cuesta College site, but the hope is that these values can be applied to other, similar sites as well. This relates primarily to the similarities in the deposition of other sites in comparison to this site. Since the site consists of alluvial deposits overlaying the Franciscan complex, the applicability to other sites with this deposition is the 
most appropriate. For other alluvial sites though, these range estimates could be used as estimates or guidelines.

Franciscan mélange which surrounds the site is known to be highly variable. The geologic map (California Conservation and California Geologic Survey, 2010) describes this mélange as a "chaotic mixture of fragmented rock masses". If the alluvium was eroded from this material and the presence of this material at the site indicates higher variability which leads to shorter (more conservative) range values. So even in sites with alluvium overlying other types of rock, these range values could be used as a conservative estimate. Isaaks and Srivastava (1989) also note that it is reasonable to infer the shape of a horizontal semi-variogram from similar data sets. So another option is to sample a few points and to infer the shape of the semi-variogram from this study's more densely sampled area.

It should also be noted that the range values in this study are based exclusively off of the velocity values. And even though the soil type and the velocity values are strongly associated, the similar sites would not necessarily need to have alluvial soils - just velocity values that are similarly distributed. This would involve a comparison between the velocity deposition of alluvial sites compared to the velocity deposition of another site/type of material. This comparison is outside the scope of this study, but the values could be used as guidelines in study's looking at these comparisons.

\subsubsection{Reliability of the Range Values}

The reliability can be checked by looking at the bias in the survey. The largest bias comes from the array direction. Since this material was deposited by rivers, the material is expected to have anisotropic velocity values (i.e. the cross-stream values are likely to be different than the down-stream values). This anisotropy can lead to changes is the range value (Isaaks and 
Srivastava, 1989). Directions that are more similar would have higher range values and viceversa. Looking at the distribution of alluvium in Figure 1.2 and seeing that Chorro creek runs in an east-west direction just below Cuesta's campus, it appears that this study's survey sampled velocity values in the cross-stream direction. Values sampled in the down-stream direction could give different range values. This anisotropy is not checked in this study and further studies would need to verify the accuracy of the range values that are presented. 


\section{Chapter 5: Conclusion}

\subsection{Summary}

The time weighted average Rayleigh wave velocities and shear wave velocities were collected along a 310 meter long profile in alluvial soil. Semi-variograms, covariance functions, and correlograms were created from this data to quantify the spatial continuity. Negative covariance values indicated that a trend could be present in the data, so the data was manipulated in four different ways to try to relieve the data of this apparent trend. However, after modeling all the options with spherical models, exponential models, and Gaussian models, the manipulated data did not appear to model the data any better than the original data. Although stationarity was violated with this data (making the covariance functions and correlograms unusable), intrinsic stationarity was assumed so that the semi-variograms would still give useful information. The spherical and Gaussian models both captured the uncertainty in the data equally well, but the Gaussian model's ranges were more conservative so these were preferred over the spherical models. Ranges of 50 meters for a 5-meter column of soil and approximately 65 meters for a 10to 15-meter column of soil when Rayleigh waves were calculated. For shear waves, the models showed ranges of 70 meters for a 5-meter column of soil and of 100 meters for a 10- to 15-meter column of soil. Using Boore's (2004) study to extrapolate deeper, the range for a 30-meter column of soil was 90 meters.

The range values for shear waves were consistently higher than those for Rayleigh waves. This implies that the Rayleigh wave values were consistently less similar than compared to the shear wave values. The most likely reason for this is because the Rayleigh wave data was calculated from an approximation involving about 10 layers, whereas the shear wave data was calculated via an inversion process and only 3 to 5 layers. The Rayleigh wave data was strictly 
from the dispersion curve, but during the inversion process iterations continue and the amount of layers can be manipulated until the values seem reasonable. This helps to constrain the shear wave velocities and exclude seemingly erroneous values which leads to shear wave values that are more similar and higher range values.

Even though the shear wave values are manipulated during the inversion process, this manipulation considers prior knowledge of the site. The geologic map, nearby borings, and nearby cross section were used to help constrain the inversion process. And, although this creates bias in the values, the goal is to have the bias based on values that are more likely to be present. These expected values which are anticipated from prior information (boring logs, geologic maps, etc.) are assumed to be more accurate than estimates from the dispersion curve. This is partly because the expected shear wave values help to constrain the data and the noise in the dispersion curve can lead to erroneous values that could influence the Rayleigh wave velocities.

Additionally, shear wave velocities are used more in practice and the range from these velocities are more applicable than the range from the less used Rayleigh wave velocities.

This gives a range of 70 meters for 5 meter depths, 100 meters for 10 to 15 meters depth, and (using Boore's 2004 study) 90 meters for 30 meters depth. These values are similar to the ranges proposed by the European code (20 to 200 meters) and on the lower, more conservative end of the ranges proposed by the Russian code (100 to 500 meters if powerline installation is ignored). Note that the ranges in these codes are based on the type of structure being built whereas this study's ranges are based on the deposition of soil only.

\subsection{Future Research}

Recommendations for future research can be grouped into two categories: confirming this study's results and seeing how well these results translate to other soil types/depositions. 
Verifying the results can be done at another site or even at the same site using a perpendicular/two-dimensional survey. A different site would allow for a comparison of the range values and a possibility to find useable covariance functions/correlograms; a study at the same site could check for anisotropy (and any corresponding changes in the range due to this anisotropy). A two-dimensional survey requires more intensive analysis (Isaaks and Srivastava, 1989, give a good summary of this) but would allow for multiple directions to be checked and for more data pairs to be analyzed with less survey points. If this approach is taken, it should be noted that Oliver and Webster (2015) advise that only 5 data points are needed in a semivariogram before the sill. This study used a small sample interval ( 5 meters) to have better chances of identifying the range and to have more data points, but since the range value is now assumed to be around 90 meters for shear waves, then this only requires a sample interval of 15 meters in order to give 5 points before the sill.

The second option is to model a site with a different soil deposition to see how well the range values match. Since the range values are based on the differences in velocity values, a future study could model a site with similar velocity values to see whether the soil deposition or the velocity values influence the range more. Of course, other sites with vastly different velocity values and deposition could also be modeled, but it seems that these sites would be less likely to show similar range values. 


\section{REFERENCES}

Achenbach, J. D. (1973). Wave Propagation in Elastic Solids. Amsterdam, The Netherlands: North-Holland Publishing.

Aki, K. (1957). Space and time spectra of stationary stochastic waves, with special reference to microtremors. BRRI, 35 , p. $415-456$

Aki, K. (1965). A note on the use of microseisms in determining the shallow structure of the Earth's crust. Geophysics, 30, 665-666.

Aki, K., \& Richards, P. (2002). Quantitative Seismology (2nd ed.). Sausalito, California: University Science Books.

Andrus, R., \& Stokoe II, K. (1996). Liquefaction Resistance of Soils from Shear wave Velocity. J. Geotech. Geoenviron. Eng. Journal of Geotechnical and Geoenvironmental Engineering, 1015-1025.

Asten, M., \& Henstridge, J. (1984). Array estimators and the use of microseisms for reconnaissance of sedimentary basins. Geophysics, 49(11), 1828-1837.

Bohling, G. (2005). Introduction to Geostatistics and Variogram Analysis [PDF document]. Retrieved from http://people.ku.edu/ gbohling/cpe940/Variograms.pdf

Boore, D. M. (2004). Estimating $\bar{V}_{s}(30)$ (or NEHRP Site Classes) from Shallow Velocity Models (Depths $<30$ m). Bulletin of the Seismological Society of America, 94(2), 591597.

Bracewell, R. N. (1978). The Fourier Transform and Its Applications. $2^{\text {nd }}$ ed. New York: McGraw-Hill.

California Conservation and California Geologic Survey [Mark O. Wiegers]. (2010). Geologic Map of the San Luis Obispo 7.5' Quadrangle San Luis Obispo County, California: A Digital Database [map]. 1:24,000. Retrieved from ftp://ftp.consrv.ca.gov/pub/dmg/rgmp/Prelim_geo_pdf/SanLuisObispo24k_preliminary.pdf

Chipping, D. (1987). The Geology of San Luis Obispo County: A Brief Description and Field Guide. San Luis Obispo, California: D. H. Chipping.

Clark, I. (1979). Practical Geostatistics. London: Applied Science.

EN 1997-2: Eurocode 7: Geotechnical Design - Part 2: Ground Investigation and Testing (English ed.). (2007). European Committee for Standardization. 
Engineering Surveys for Construction. Basic Provisions (SNiP 11-02-96 ed.). (1996). Russian Federation.

Facciorusso, J., Uzielli, M., \& Mayne, P. (2010). Spatial characterization of Vs at Amherst NGES site from SCPT using Bayesian kriging. Conference paper at 2nd International Symposium on Cone Penetration Testing, Huntington Beach, CA.

Foti, S., Lai, C., Rix, G., \& Strobbia, C. (2014). Surface Wave Methods for Near-Surface Site Characterization. CRC Press.

Geogiga Surface Plus (Version 7.1) [Software]. (2012). Calgary, Alberta, Canada: Geogiga Technology Corporation.

Google Maps. (2015). [Cuesta College, San Luis Obispo, California] [Map]. Retrieved from https://www.google.com/maps/place/Cuesta+College/@35.2249348,120.1304288,9z/data $=! 4 \mathrm{~m} 2 ! 3 \mathrm{~m} 1$ !1s0x80ece51406b45c83:0xf478e2d29c4fed3a

Hayashi, K. (2015). Array Microtremor (SPAC); past, present, future \& comments regarding InterPacific project. Unpublished presentation, Post-SSA Forum, California Institute of Technology, Pasadena, CA.

Horike, M. (1985). Inversion of phase velocity of long period microtremors to the S-wave velocity structure down to the basement of urbanized areas. J Phys Earth, 33, 59-96.

Hudson, J. A. (1980). The Excitation and Propagation of Elastic Waves. Cambridge: Cambridge University Press.

Isaaks, E., \& Srivastava, R. (1989). An Introduction to Applied Geostatistics. New York: Oxford University Press.

Iqbal, J., Thomasson, J., Jenkins, J., Owens, P., \& Whisler, F. (2005). Spatial Variability Analysis of Soil Physical Properties of Alluvial Soils. Soil Science Society of America Journal, 69, 1338-1350. doi:10.2136/sssaj2004.0154

Joyner, W. B., R. E. Warrick, \& Fumal, T. E. (1981). The effect of Quaternary alluvium on strong ground motion in the Coyote Lake, California, earthquake of 1979, Bulletin of the Seismolocial Society of America, 71, 1333-1349.

Lai, C. G. (2005). Surface waves in dissipative media: Forward and inverse modeling. Surface Waves in Geomechanics: Direct and Inverse Modelling for Soils and Rocks (C.G. Lai and K. Wilmanski, eds.). Wien, New York: Springer-Verlag.

Lai, C. G., Foti, S., \& Rix, G. J. (2005). Propagation of data uncertainty in surface wave inversion. J Environ Eng Geophys, 10(2), 219-228. 
Lamb, H. (1904). On the propagation of tremors over the surface of an elastic solid. Philos Trans Roy Soc Lond Ser A, 203, 1-42.

Louie, J. N. (2001). Faster, Better: Shear wave Velocity to 100 Meters Depth from Refraction Microtremor Arrays. Bulletin of the Seismological Society of America, 347-364.

Malagnini, L., Rovelli, A., Hough, S., \& Seeber, L. (1993). Site Amplification Estimates in the Garigliano Valley, Central Italy, Based on Dense Array Measurements of Ambient Noise. Bulletin of the Seismological Society of America, 83(6), 1744-1755.

Marosi, K. T., \& Hiltunen, D. R. (2004). Characterization of SASW phase angle and phase velocity measurement uncertainty. Geotech Test J, 27(2), 205-213.

Matheron, G. (1963). Les variables régionalisées et leur estimation. Paris: Masson.

Matheron, G. (1965). Principles of Geostatistics. Economic Geology, 58, 1246-1266.

Moss, R. (2008). Quantifying Measurement Uncertainty of Thirty-Meter Shear wave Velocity. Bulletin of the Seismological Society of America, 1399-1411.

Oliver, M., \& Webster, R. (2015). Basic Steps in Geostatistics:The Variogram and Kriging. Springer.

O'Neill, A. (2003). Full-waveform reflectivity for modelling, inversion and appraisal of seismic surface wave dispersion in shallow site investigations. University of Western Austrailia.

Parker, R. L. (1977). Understanding inverse theory. Ann Rev Earth Planet Sci, 5, 35-64.

Rayleigh, J. (1877). The Theory of Sound (1st ed.). London: Macmillan and Co.

Rix, G., \& Leipski, E. (1991). Accuracy and Resolution of Surface Wave Inversion. Recent advances in instrumentation, data acquisition and testing in soil dynamics: Am. Soc. Civil Eng., 17-32.

Santamarina, J., Klein, K., \& Fam, M. (2001). Soils and Waves. Chichester: J. Wiley \& Sons.

Schwanghart, W. (2010) Variogramfit (Version 1.5) [MATLAB code]. Available at http://www.mathworks.com/matlabcentral/fileexchange/25948-variogramfit (Accessed July 2015)

Shekhar, S. (2008). Kriging. In H. Xiong (Ed.), Encyclopedia of GIS. New York: Springer.

Stein, S., \& Stein, J. (2014). Nature Bats Last. In Playing Against Nature: Integrating Science and Economics to Mitigate Natural Hazards in an Uncertain World. John Wiley \& Sons. 
Stover, Christopher \& Weisstein, Eric W. "Fréchet Derivative." From MathWorld--A Wolfram Web Resource. http://mathworld.wolfram.com/FrechetDerivative.html

Strang, G. (1980). Linear Algebra and Its Applications (2nd ed.). New York: Academic Press.

Telford, W., \& Geldart, L. (1990). Applied Geophysics (2nd ed.). Cambridge: Cambridge University Press.

Tezcan, S., Keceli, A., \& Ozdemir, Z. (2006). Allowable Bearing Capacity of Shallow Foundations Based on Shear Wave Velocity. Geotechnical and Geological Engineering Geotech Geol Eng, 24, 203-218.

Thitimakorn, T. (2013). Development of a NEHRP site classification map of Chiang Mai city, Thailand, based on shear wave velocity using the MASW technique. J. Geophys. Eng. Journal of Geophysics and Engineering, 045007-045007.

Tuomi, K. E., \& Hiltunen, D. R. (1996). Reliability of the SASW method for determination of the shear modulus of soils. Proceedings of Uncertainty in Geologic Environment: From Theory to Practice. American Society of Civil Engineers. Shacklford, Reston, Virginia. 1125-1237.

Tokimatsu, K. (1995). Geotechnical Site Characterization using Surface Waves. Proceedings of 1st International Conference on Earthquake Geotechnical Engineering, 3, 1333-1368.

Virieux, J., \& Operto, S. (2009). An overview of full-waveform inversion in exploration geophysics. Geophysics, 74(6), WCC127-WCC152. doi:10.1190/1.3238367

Webster, R. (2000). Is soil variation random? Geoderma, 97, 149-163.

Webster, R., \& Oliver, M. A. (1992). Sample adequately to estimate variograms of soil properties. Journal of Soil Science, 40, 493-496.

Wills, C. J., \& Clahan, C. B. (2006). Developing a map of geologically defined site-condition categories for California, Bull. Seismo. Soc. Am., 96(4A):1483-1501. 


\section{APPENDICES}

\section{Appendix A: Earth Systems Pacific's Boring Logs for the Cuesta College Sewer Line and}

\section{Pipe Bridge Replacement Project (2010)}

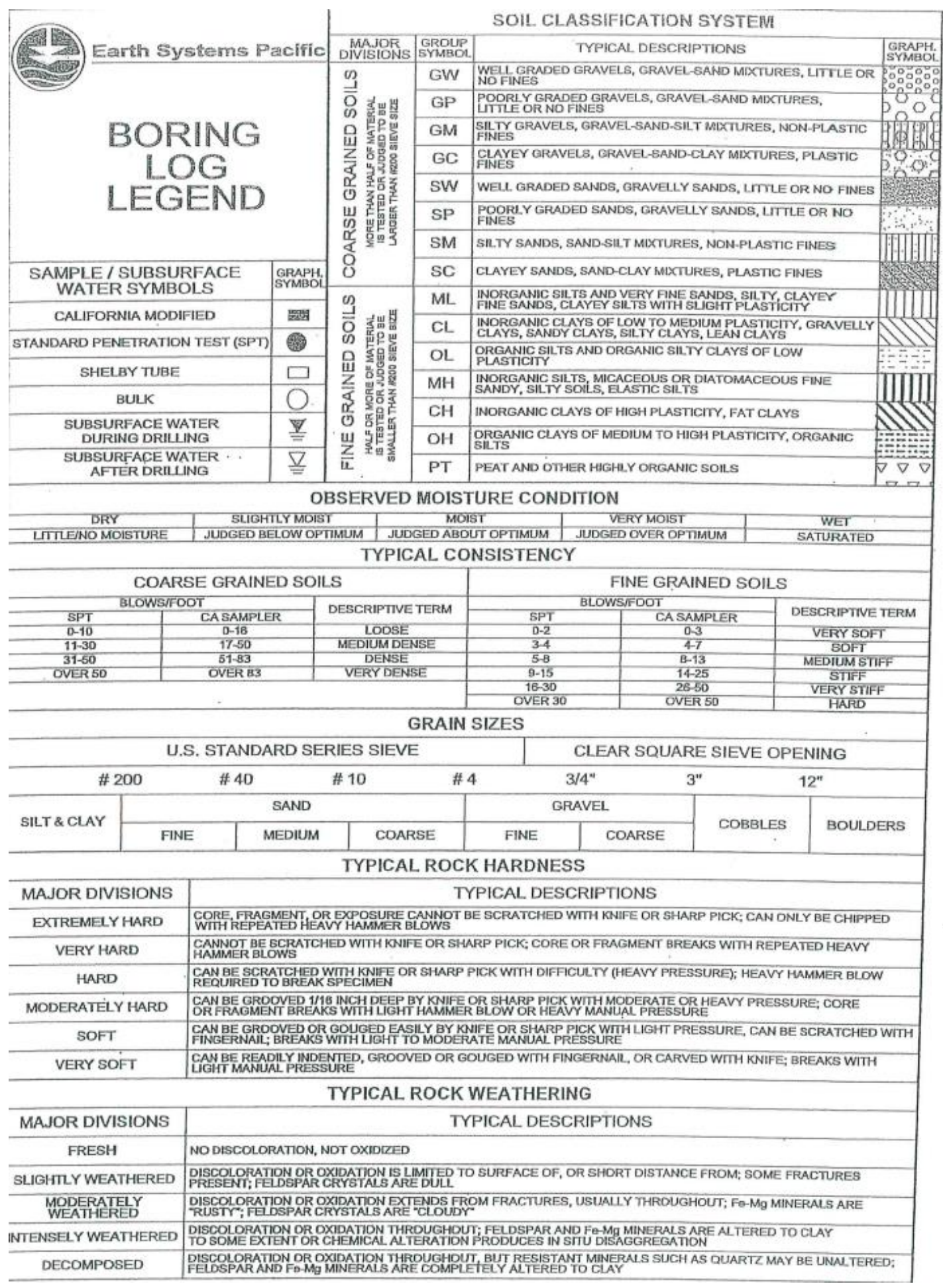


Earth Systems Pacific

LOGGED BY: A. Hinkle

Boring No. 1

PAGE 1 OF 1

DRILL RIG: Mobile B-53

AUGER TYPE: 8" Hollow Stem

JOB NO.: SL-15726-SB

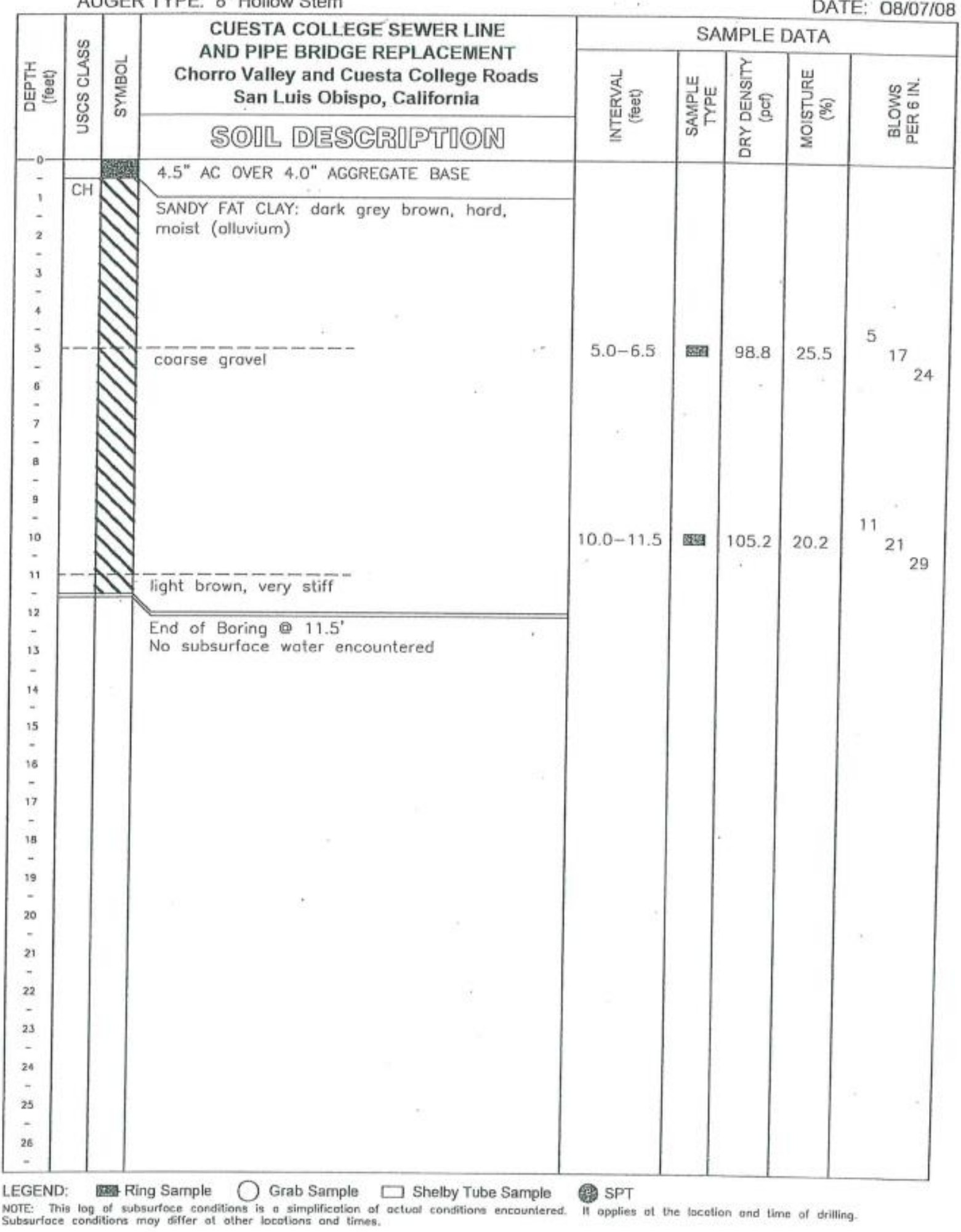




\section{Earth Systems Pacific}

LOGGED BY: A. Hinkle

Boring No. 2

PAGE 1 OF 1

DRILL RIG: Mobile B-53

AUGER TYPE: 8" Hollow Stem

JOB NO: SL-15726-SB

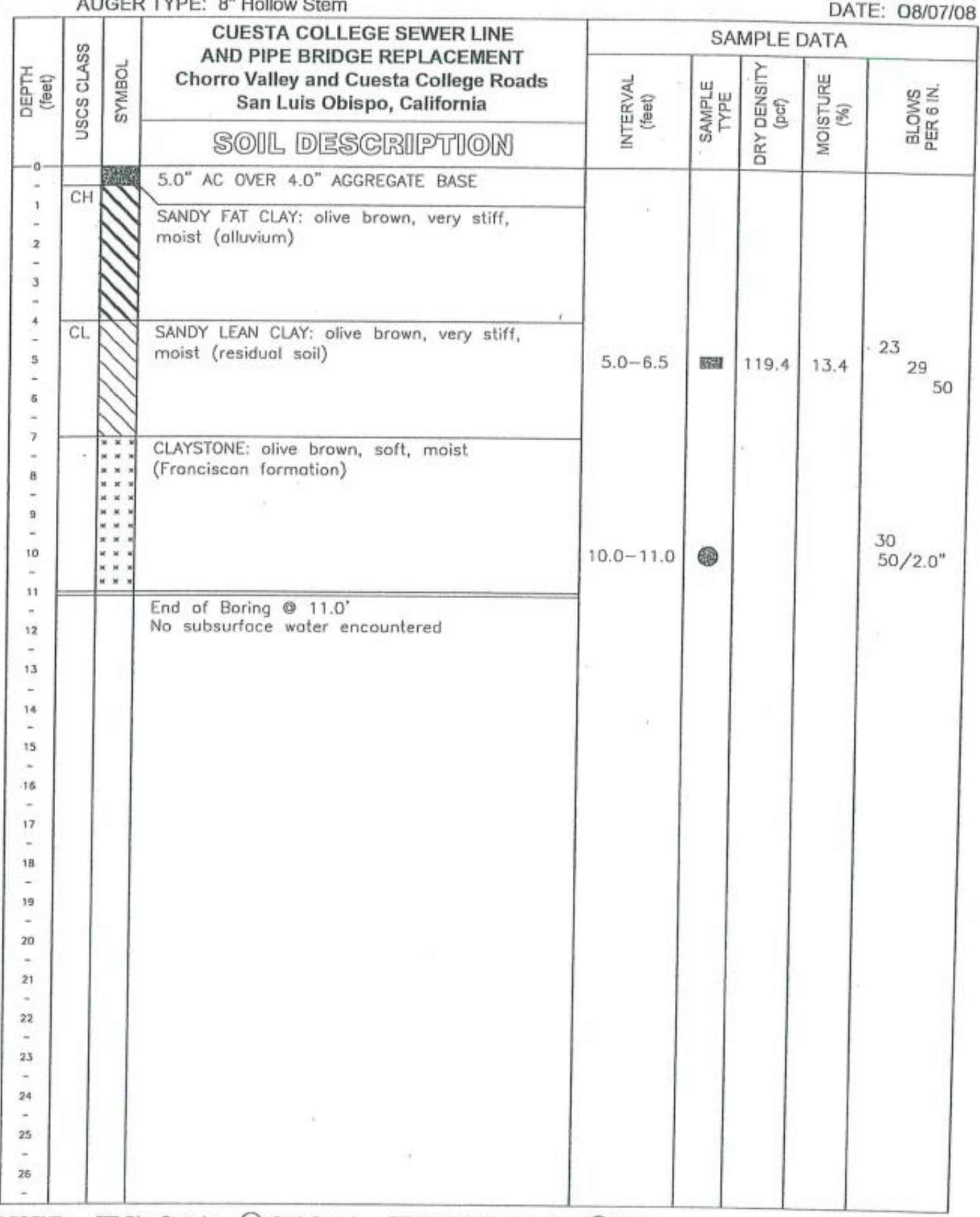

LEGEND: Ring Sample $\bigcirc$ Grab Sample $\square$ Shelby Tube Sample

2.2 SPT

NOTE: This log of subsurfoce canditions is a simplification of octual conditions encountered, It opplies of the lacation and time of drilling.
Subsurface canditions may differ at other locotions end times. 


\section{Earth Systems Pacific}

LOGGED BY: A. Hinkle

Boring No. 3

PAGE 1 OF 1

DRILL RIG: Mobile B-53

JOB NO.: SL-15726-SB

AUGER TYPE: $8^{n}$ Hollow Stem

DATE: 08/07/08

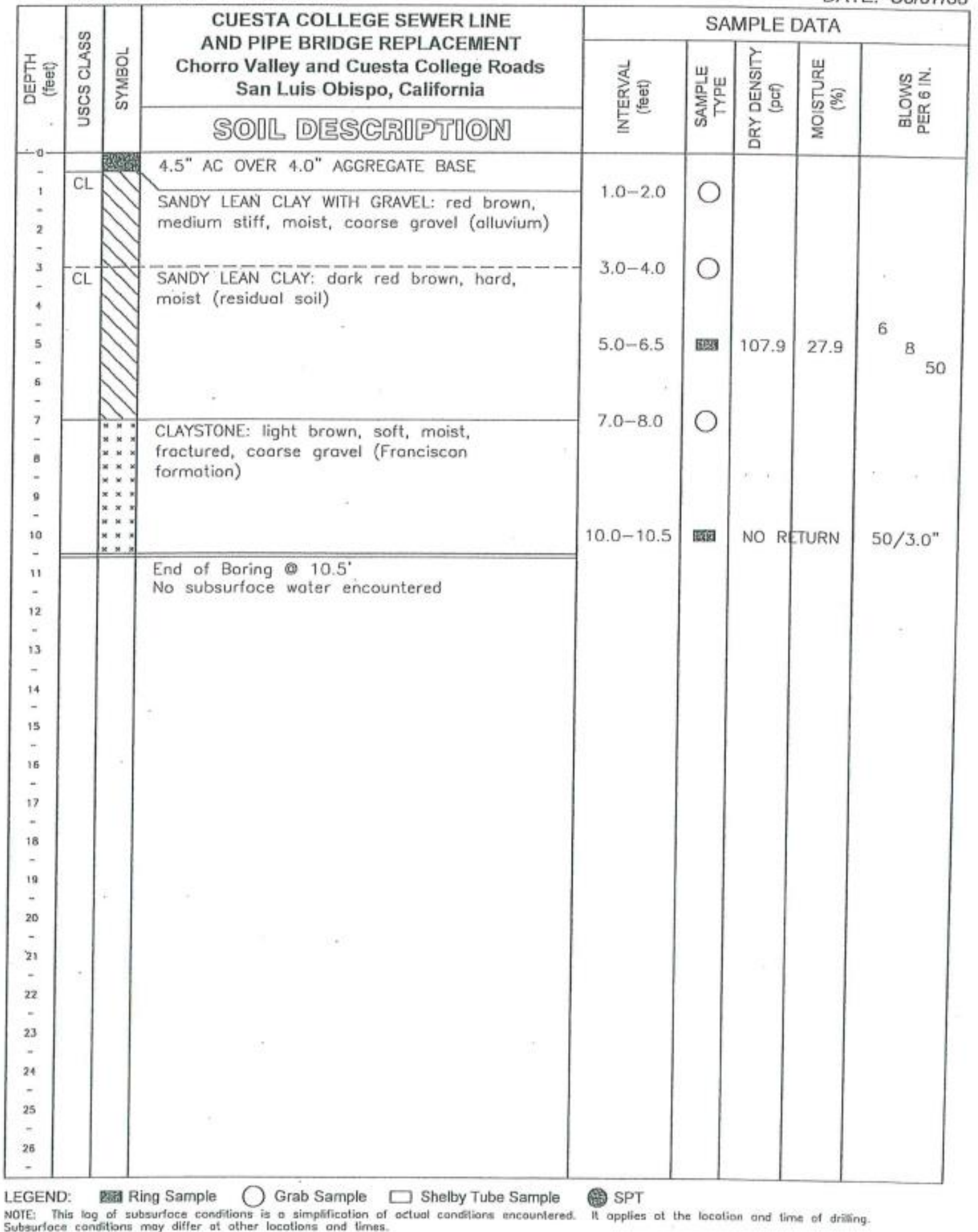




\section{Earth Systems Pacific}

LOGGED BY: D. Burns

DRILL RIG: Mobile B-53

Boring No. 4

PAGE 1 OF 1

AUGER TYPE: 8" Hollow Stem

JOB NO:: SL-15726-SB

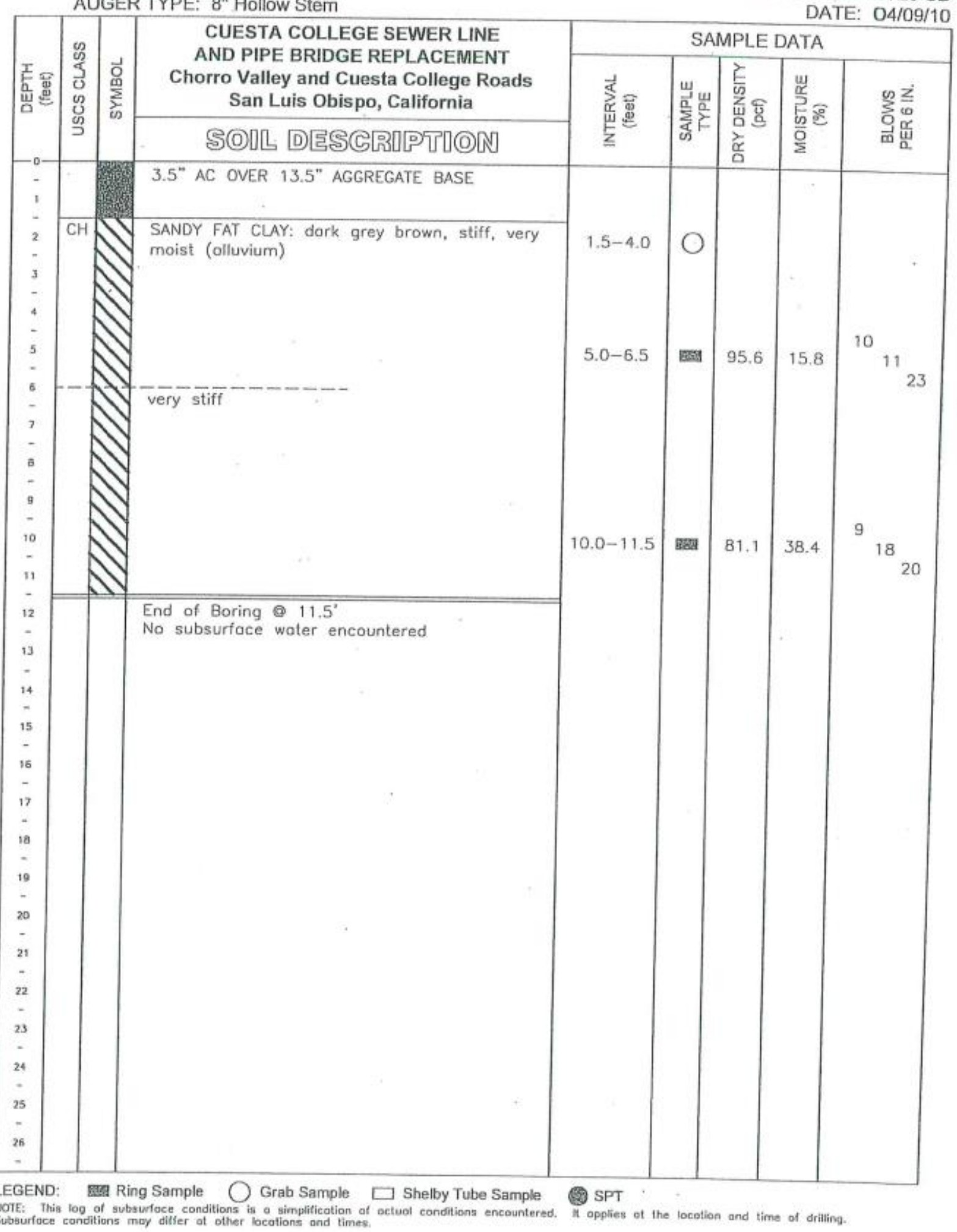


Earth Systems Pacific

LOGGED BY: D. Burns

Boring No. 5

PAGE 1 OF 1

DRILL RIG: Mobile B-53

AUGER TYPE: 8" Hollow Stem

JOB NO.: SL-15726-SB

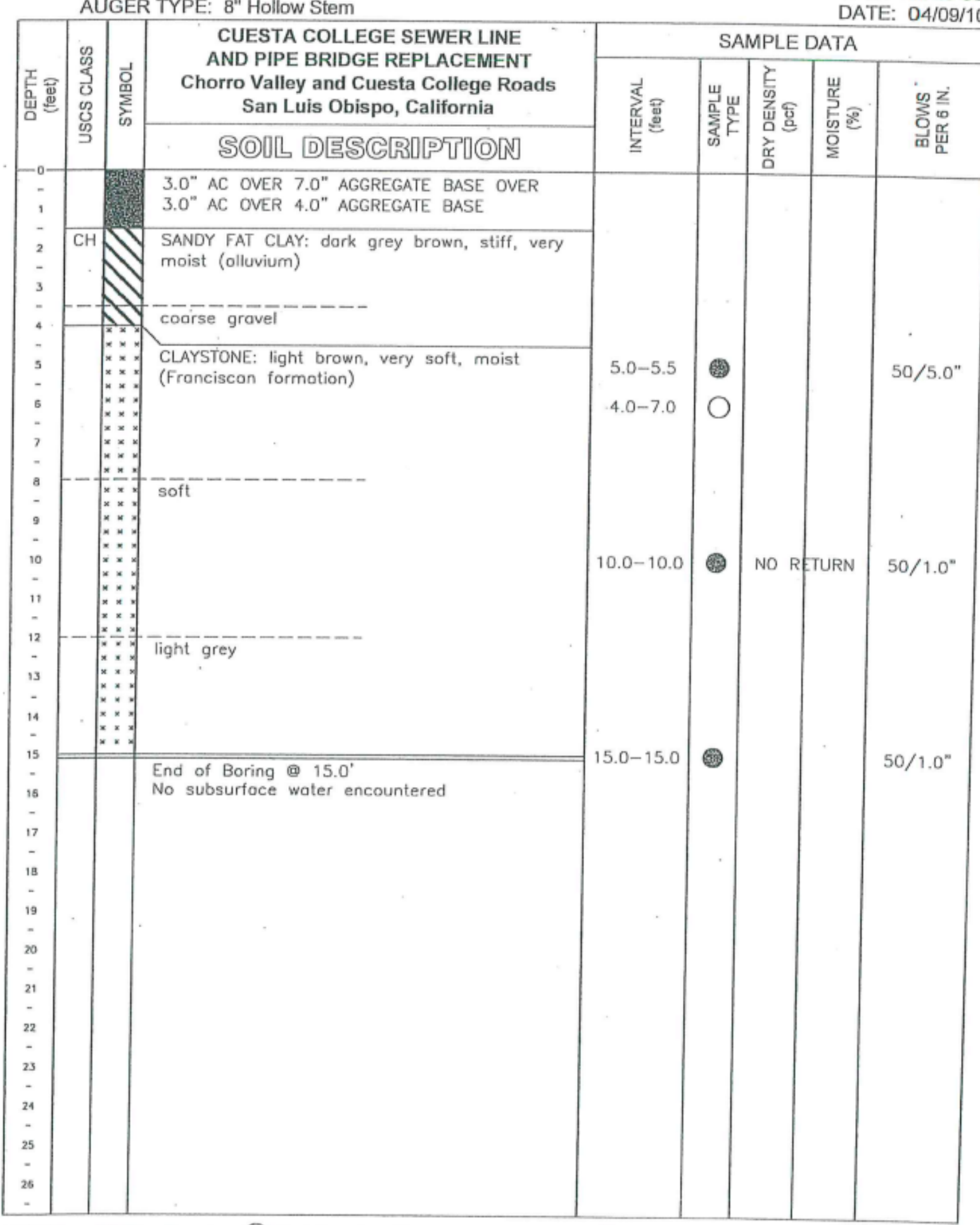

LEGEND: 맚 Ring Sample $\bigcirc$ Grab Sample $\square$ Shelby Tube Sample NOTE: This log of subsurfoce conditions is a simplificotion of actual conditions encountered. It opplies at the locotion and time of drilling 
AUGER TYPE: 8 " Hollow Stem DATE: $04 / 09 / 10$

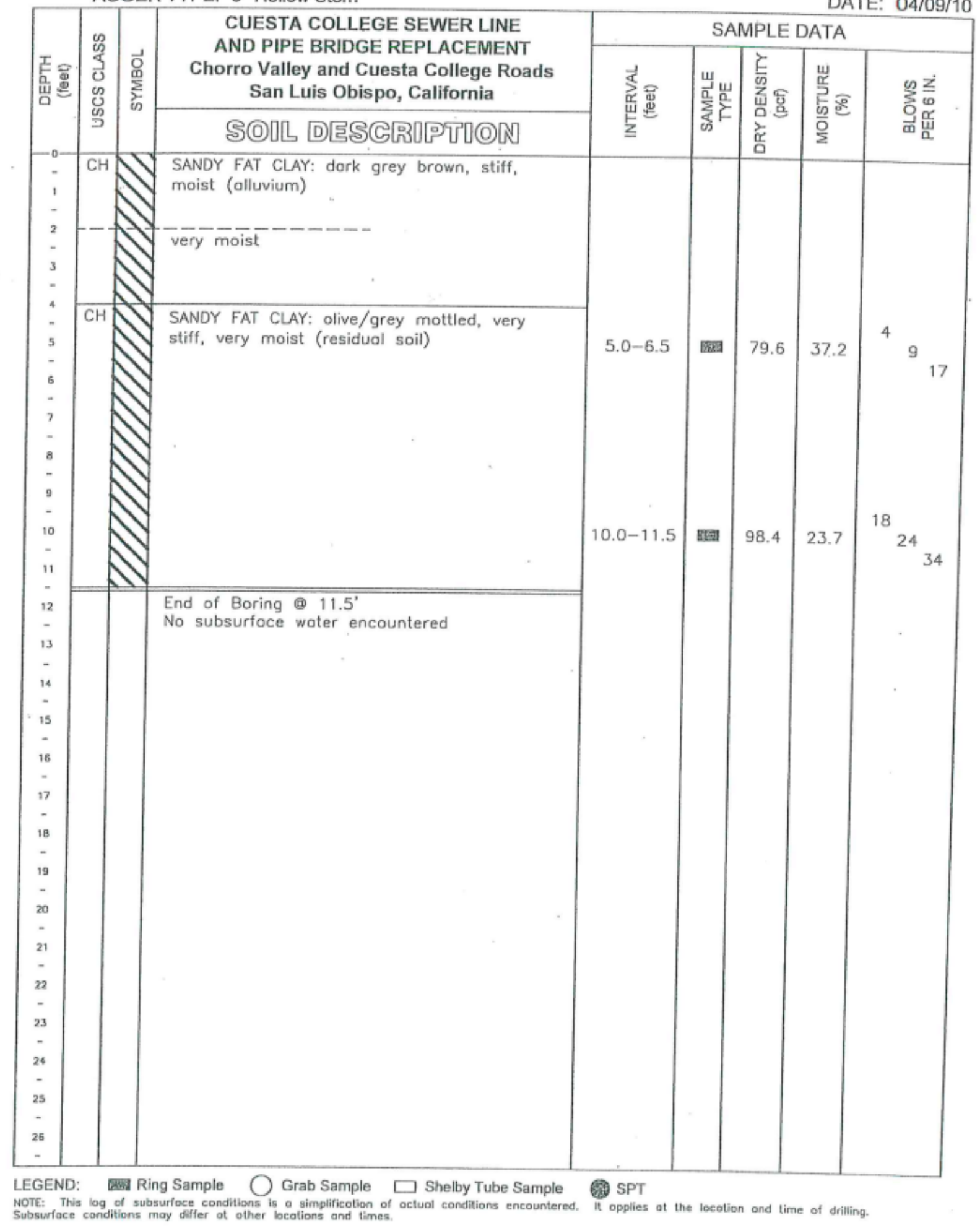




\section{Earth Systems Pacific}

LOGGED BY: D. Burns

Boring No. 7

PAGE 1 OF 1

DRILL RIG: Mobile B-53

AUGER TYPE: 8 " Hollow Stem

JOB NO.: SL-15726-SB

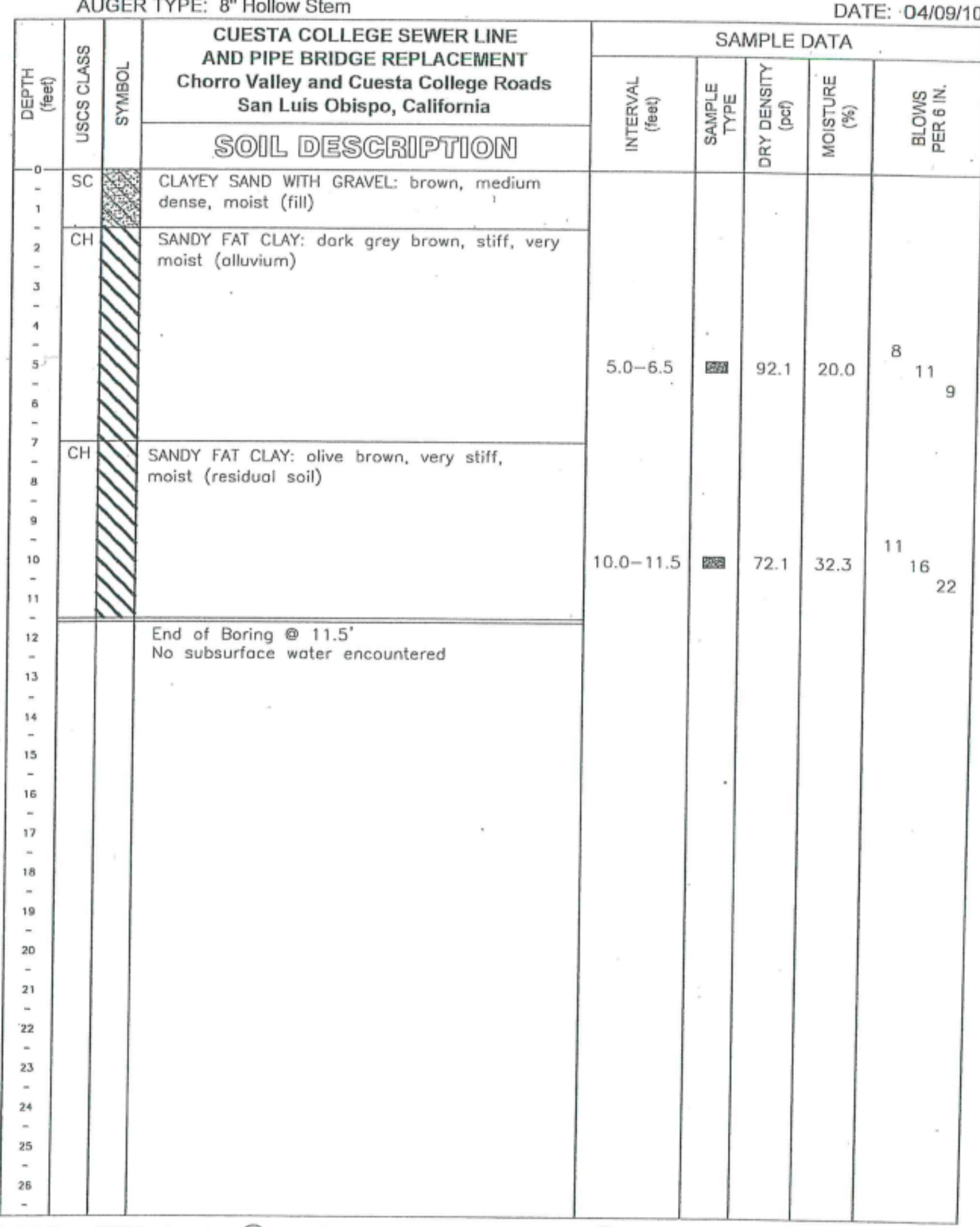

LEGEND: $\quad \square$ Shelby Tube Sample

NOTE: This log of subsurfoce conditions is o simplificotion of actuol conditions encountered. It applies of the locotion and time of drilling.
Subsurfoce conditions moy deffer ot other locations and times. 
AUGER TYPE: 8" Hollow Stem

JOB NO: SL-15726-SB

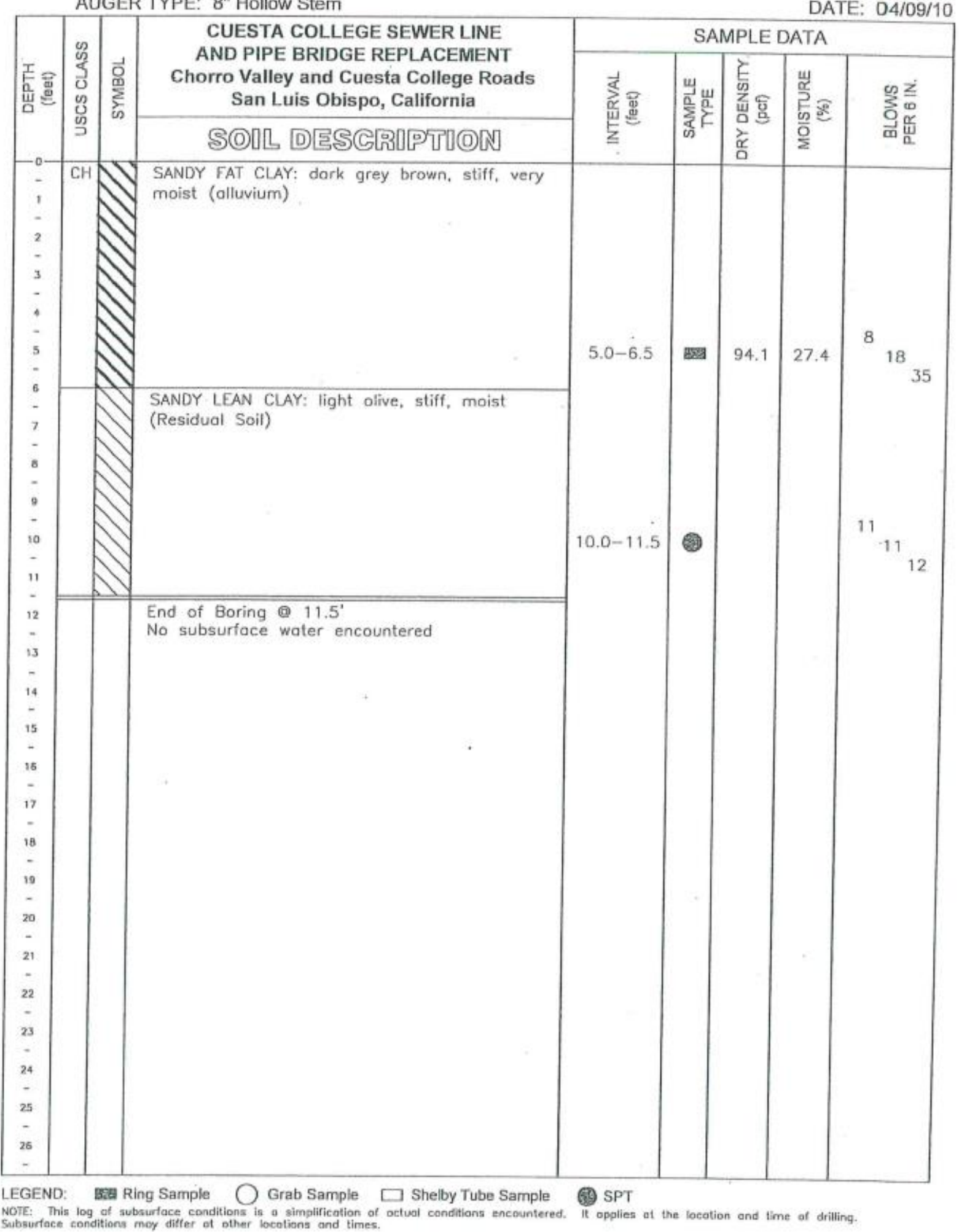




\section{Appendix B: SPAC Results for Each Survey}

Each survey is referenced based on the center of the array in relation to the reference stake (see Figure 3.2) where northern surveys are negative values and southern are positive values. For example, the array whose center is 55 meters south of the reference stake is denoted as the 55 meter survey. In the following figures, $V_{R}$ represents the Rayleigh wave velocity and $V_{S}$ represents the shear wave velocity. The figures below are ordered, from left to right, as the $V_{R}$ dispersion curve, the $V_{R}$ profile, the $V_{S}$ profile, the inversion dispersion curve, and the inversion error iterations.

\section{-20 Meter Survey}

$V_{R}$ Dispersion Curve

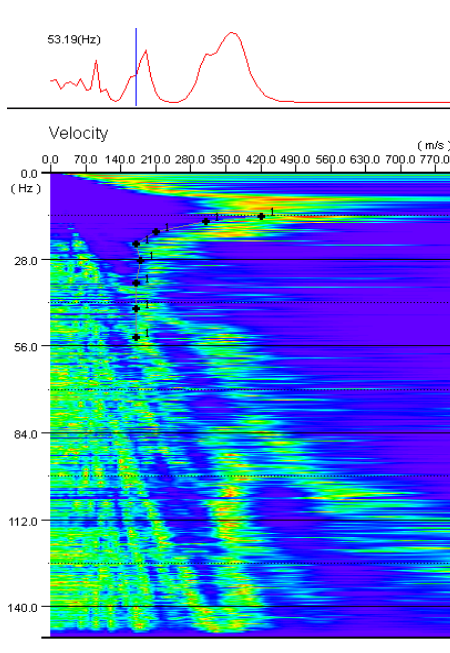

Dispersion Curves

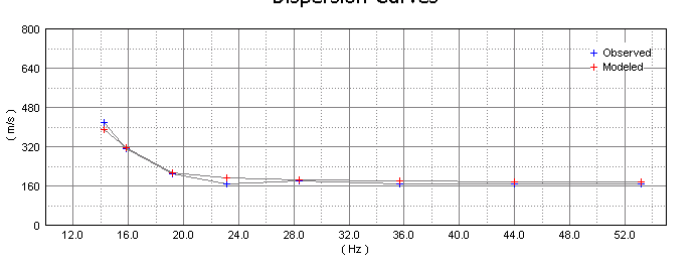

$V_{R}$ Profile
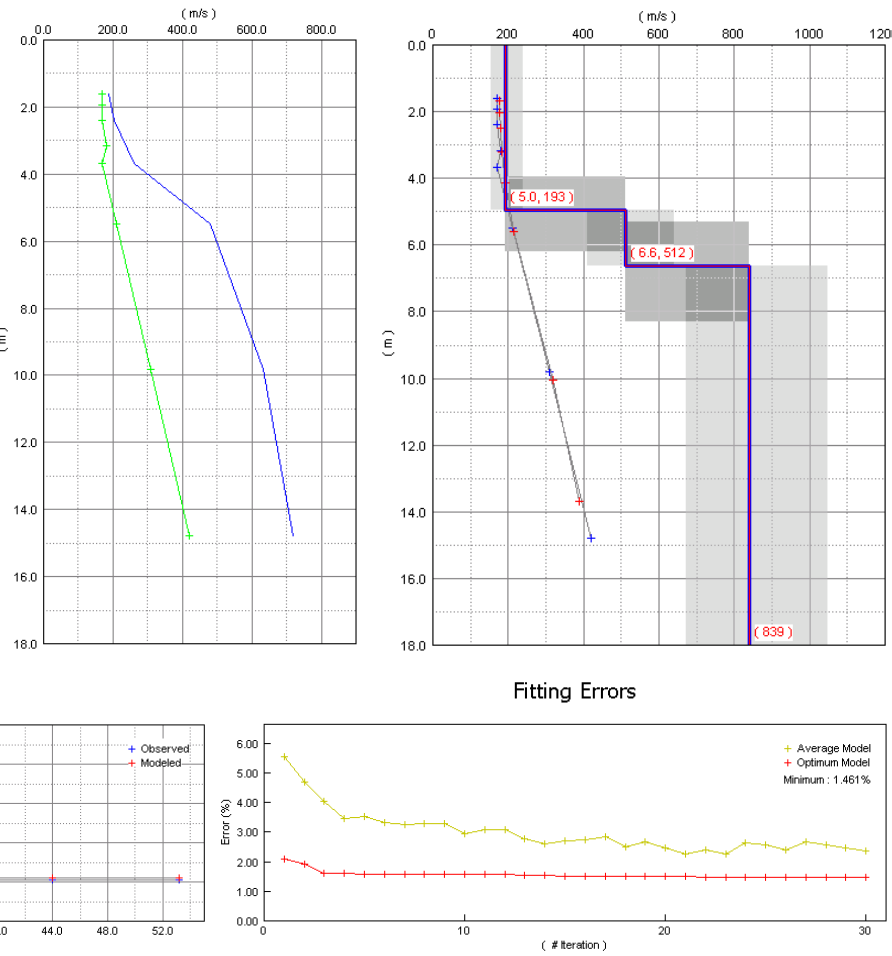
-15 Meter Survey

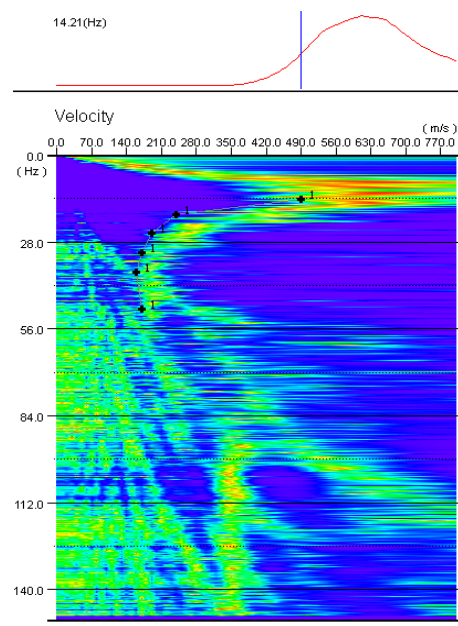

Dispersion Curves

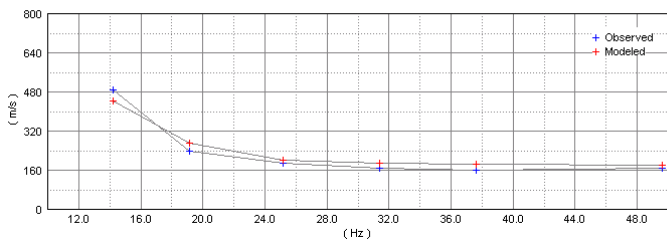

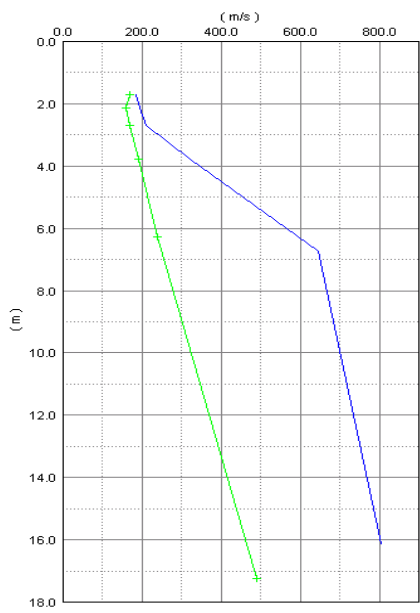

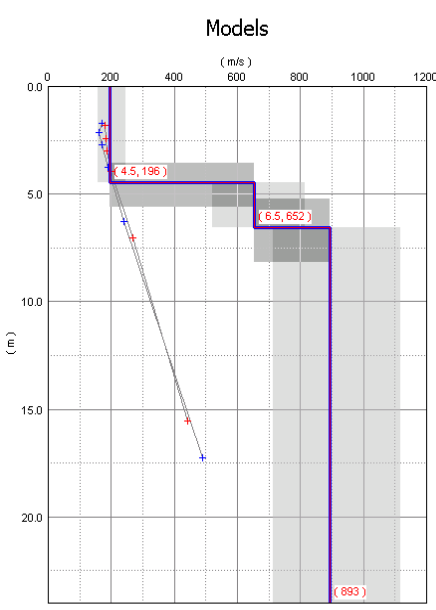

Fitting Errors

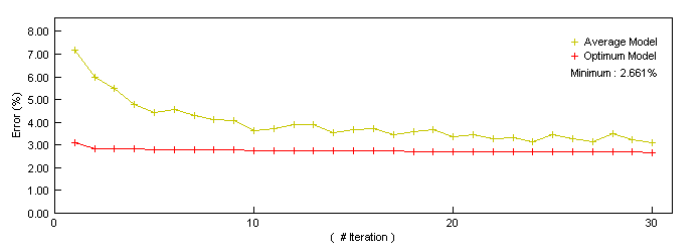

-10 Meter Survey
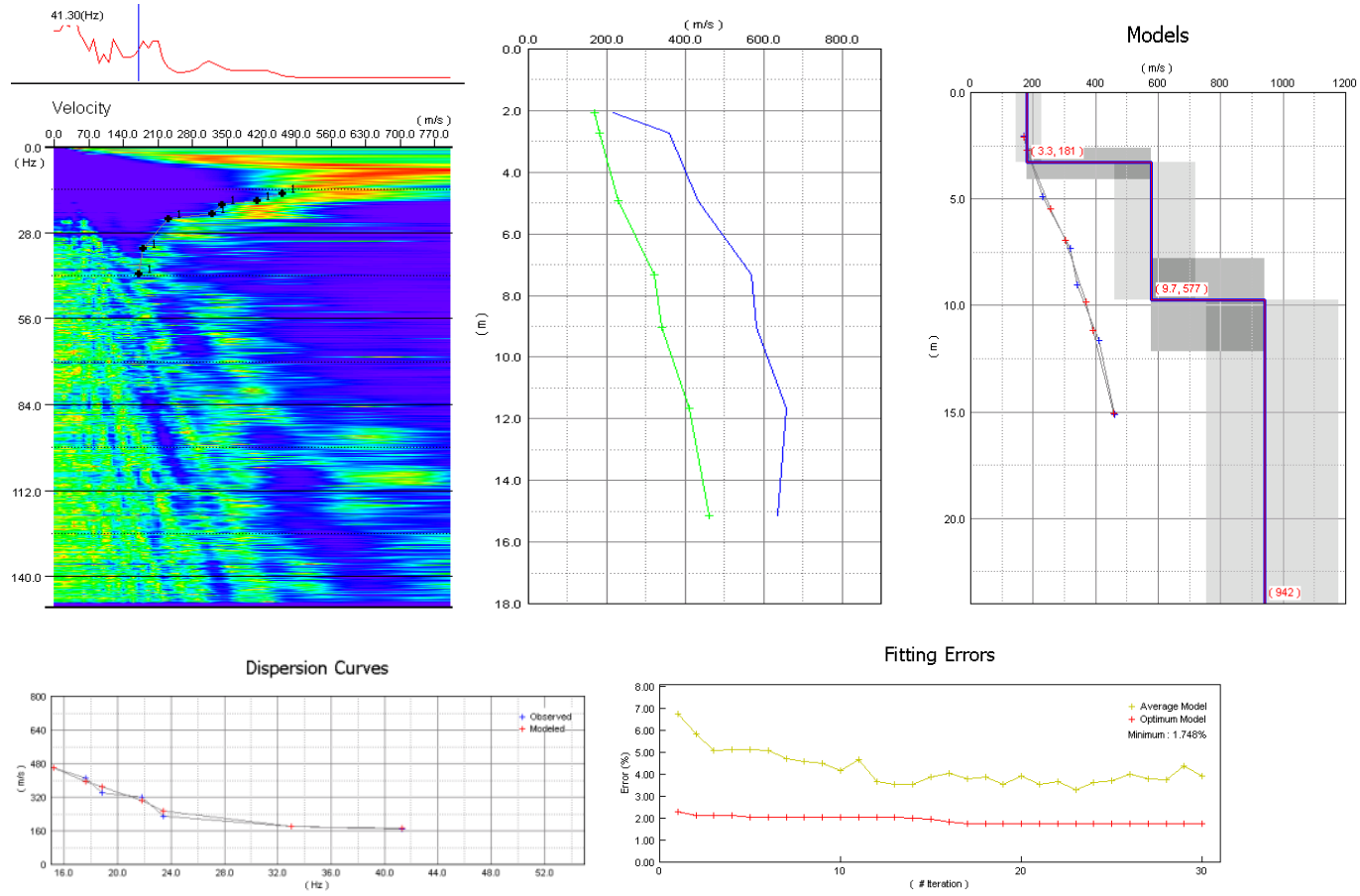


\section{-5 Meter Survey}
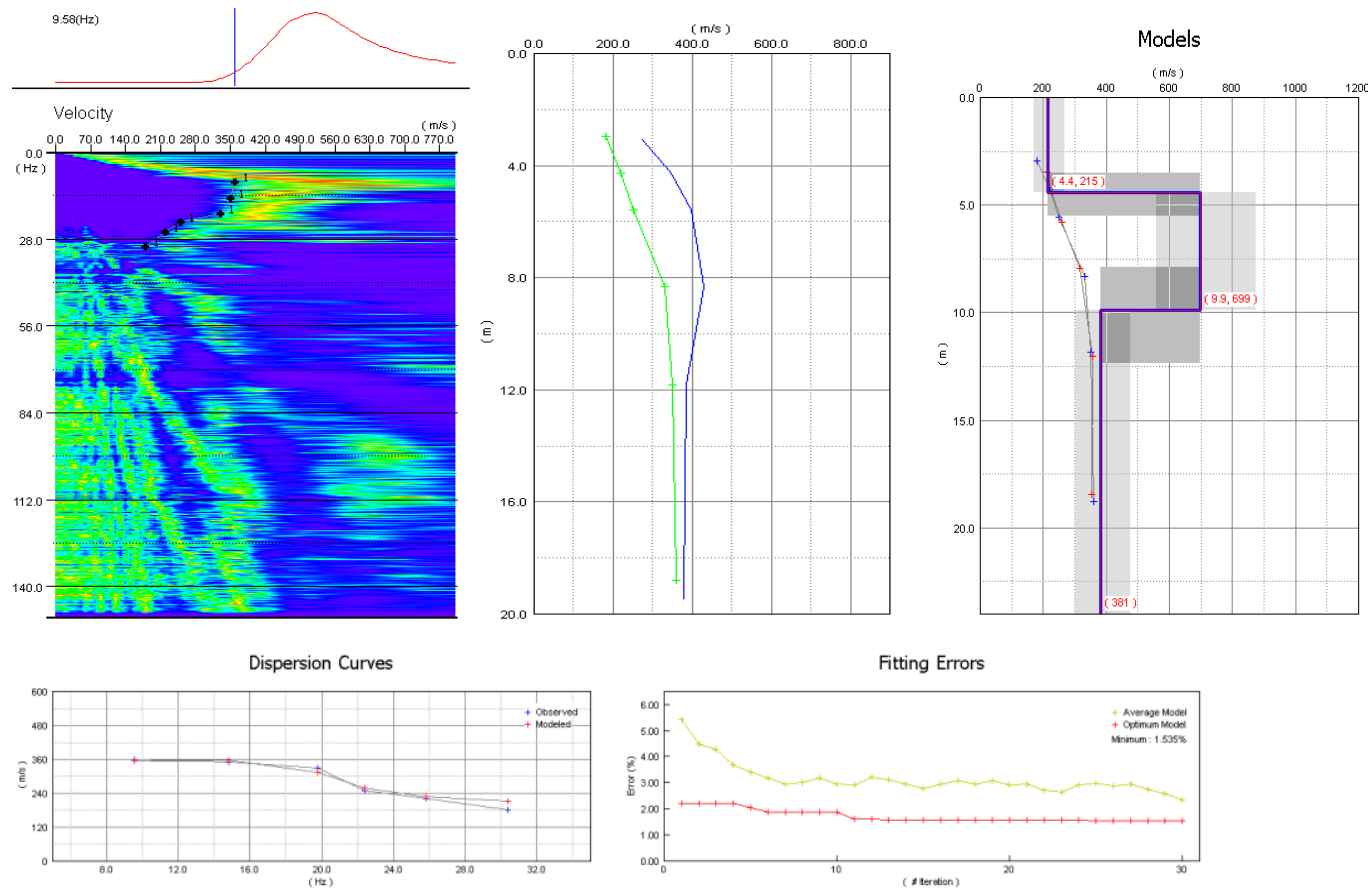

\section{o Meter Survey}
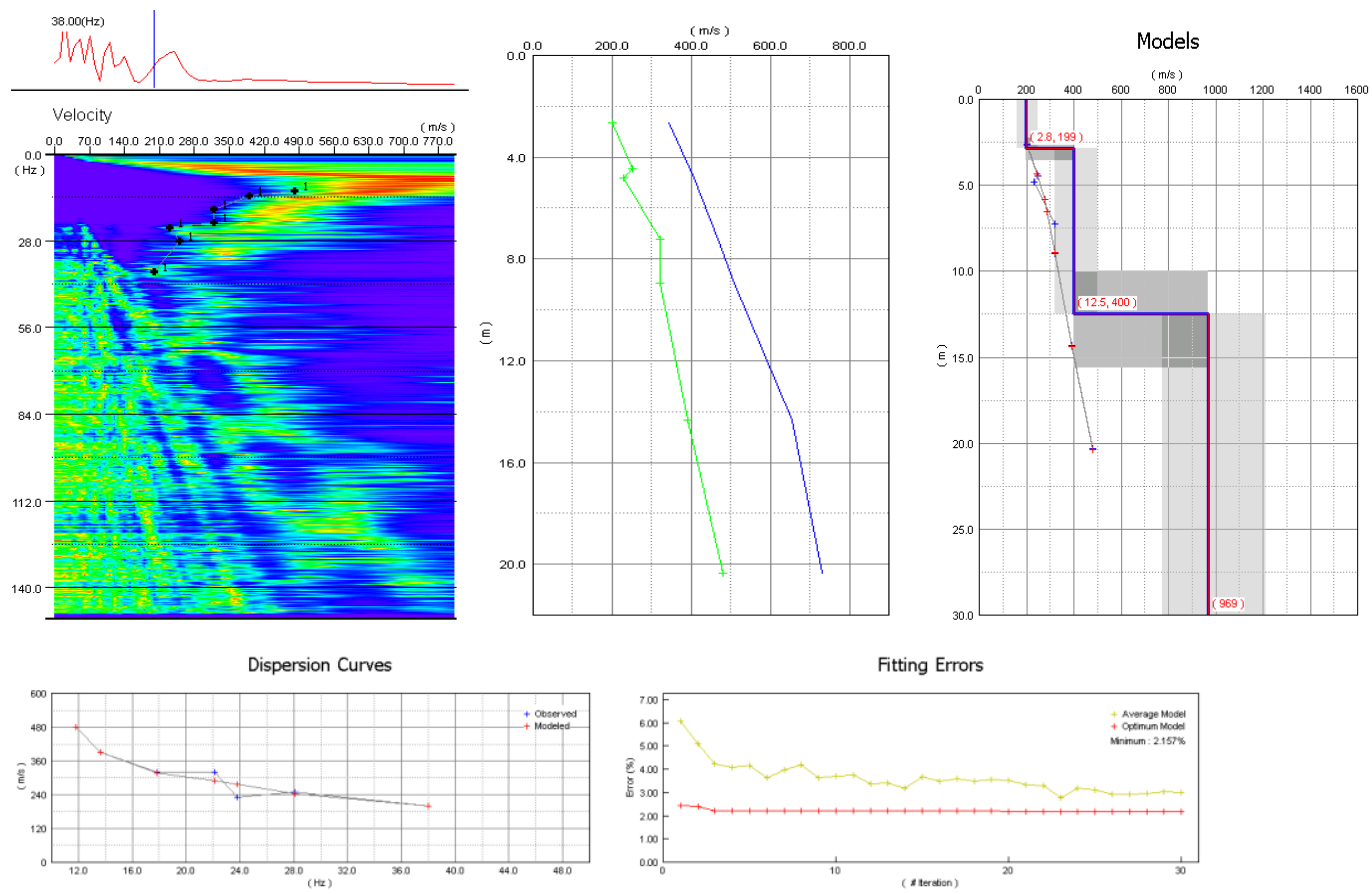


\section{Meter Survey}
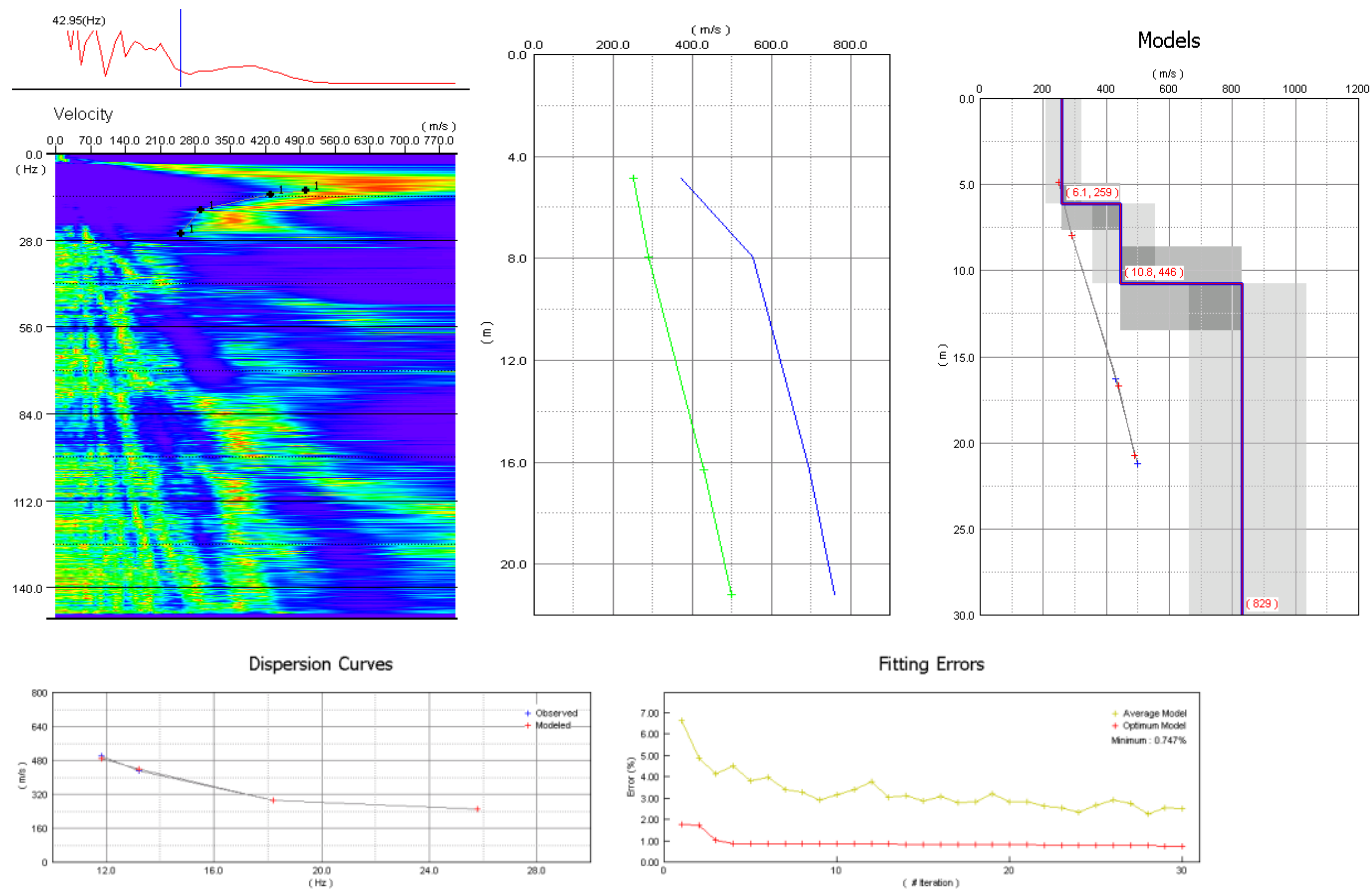

\section{Meter Survey}
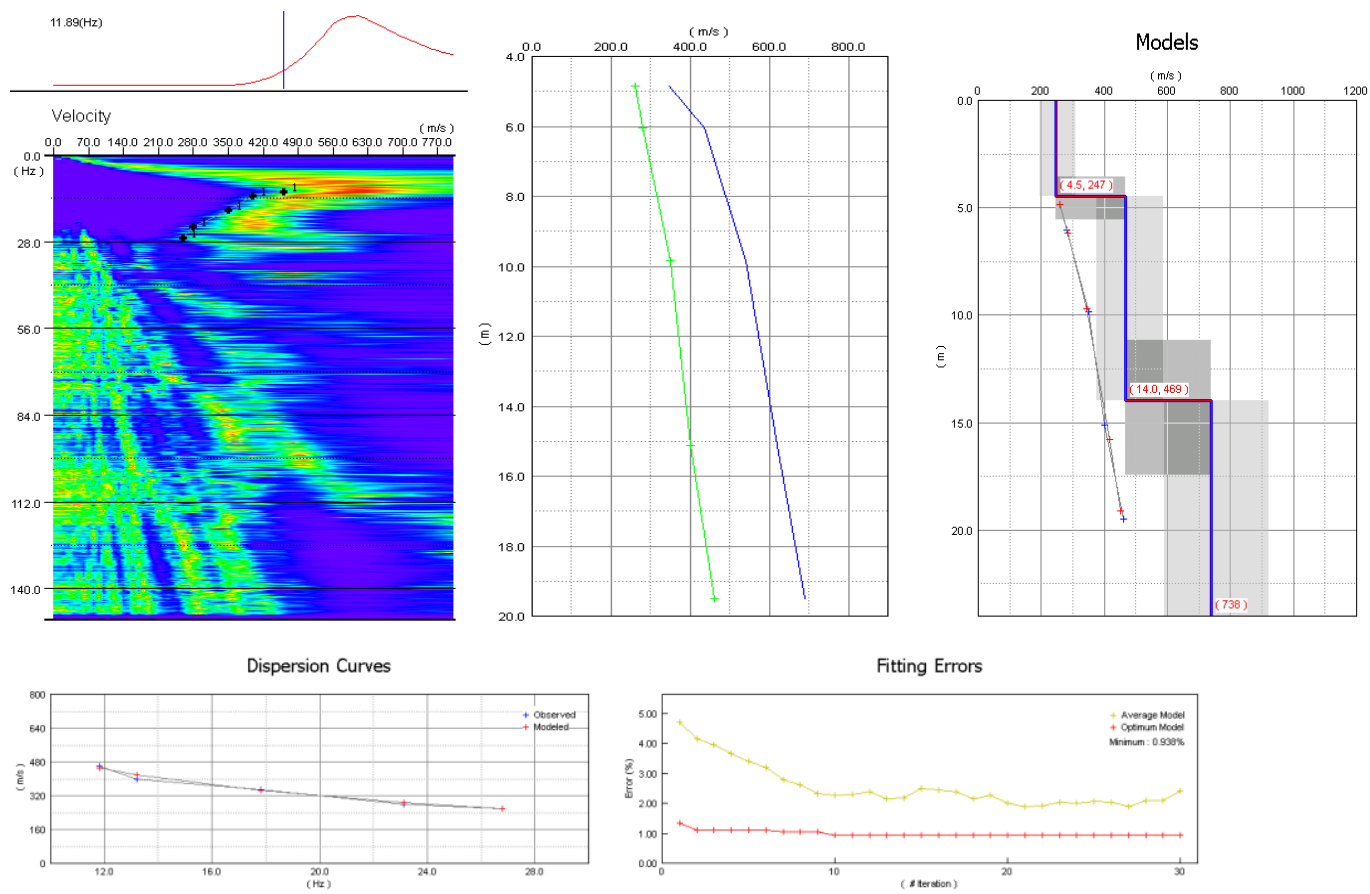


\section{Meter Survey}
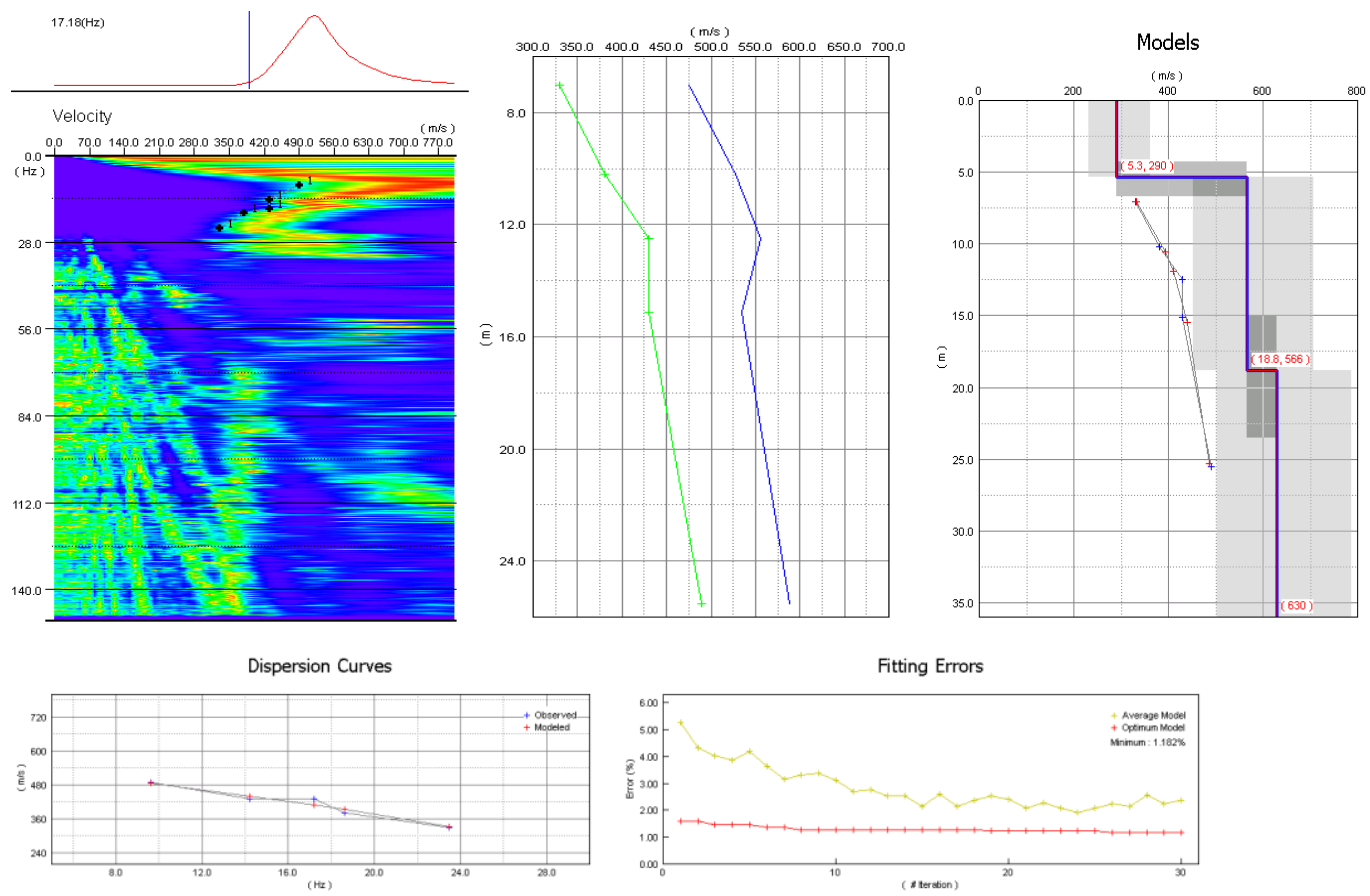

\section{Meter Survey}
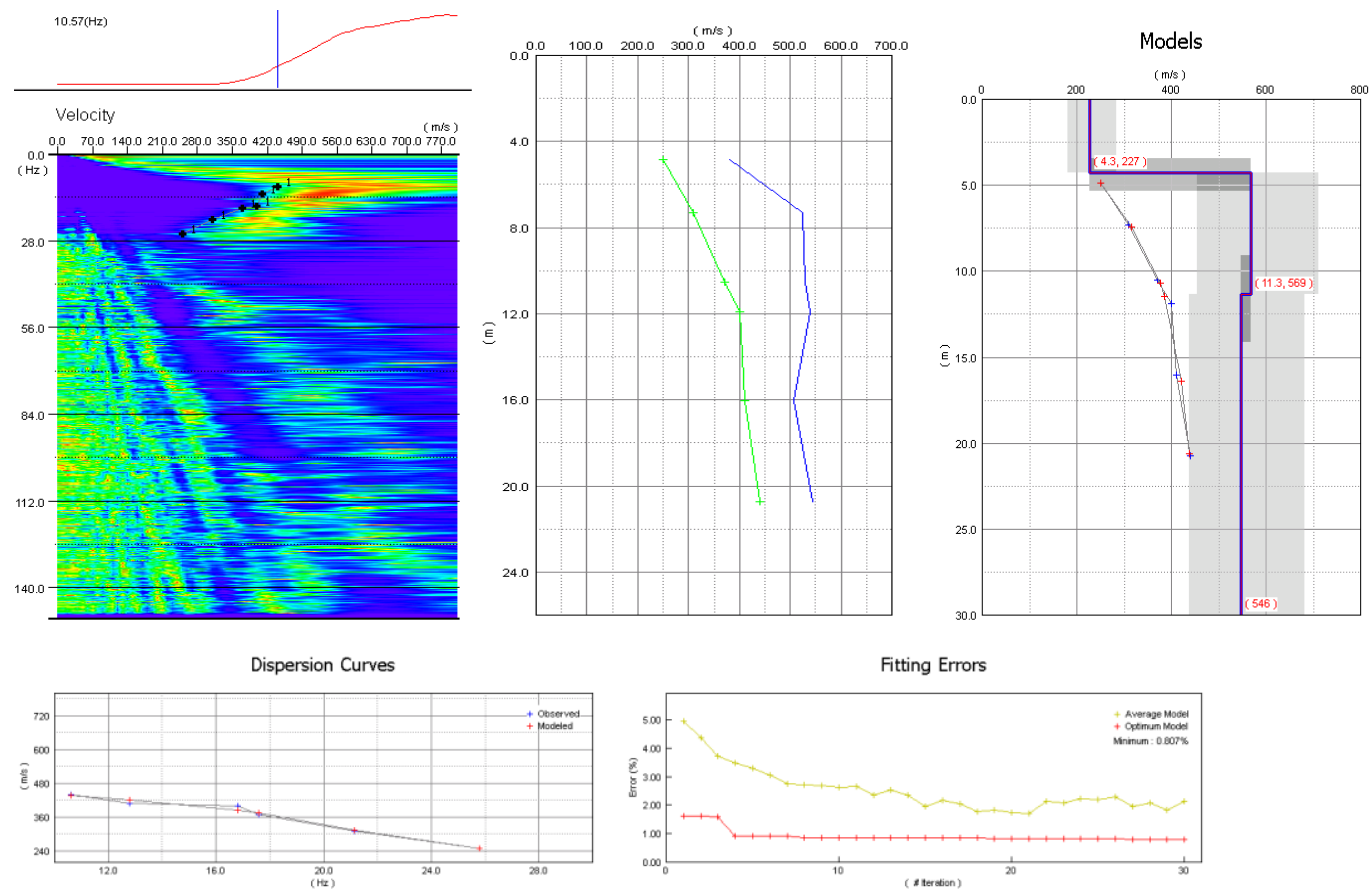


\section{Meter Survey}
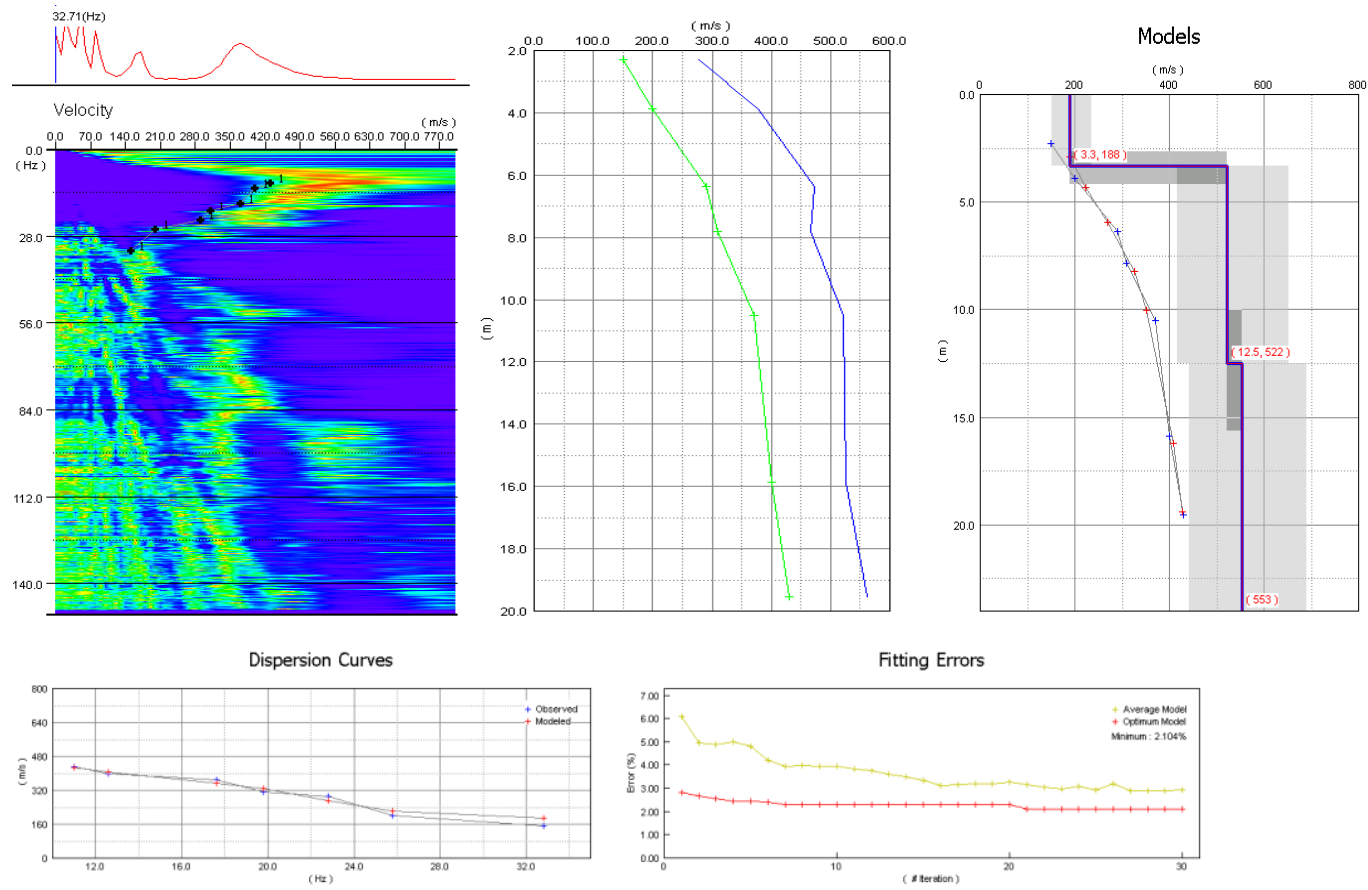

\section{Meter Survey}
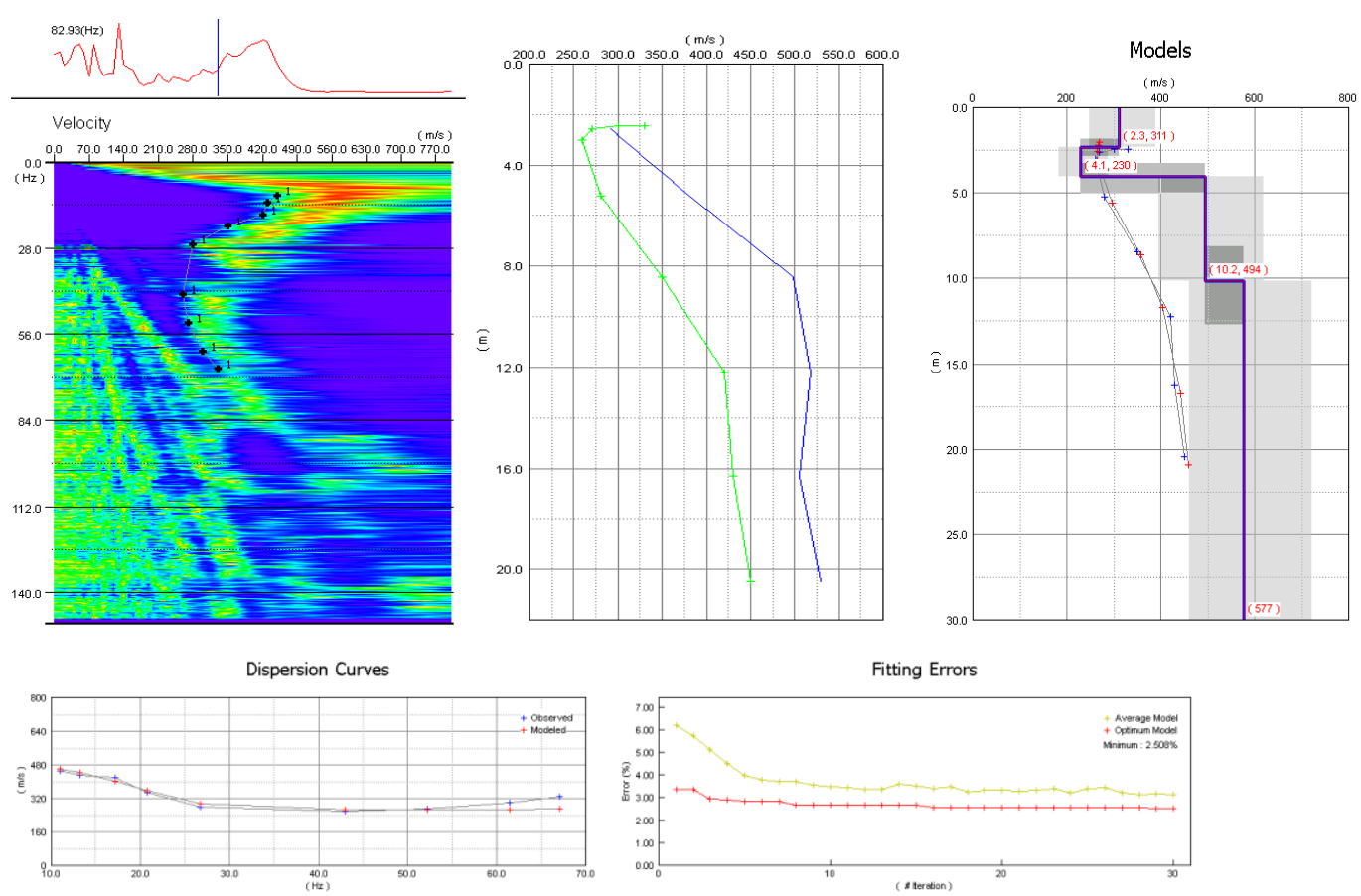


\section{Meter Survey}
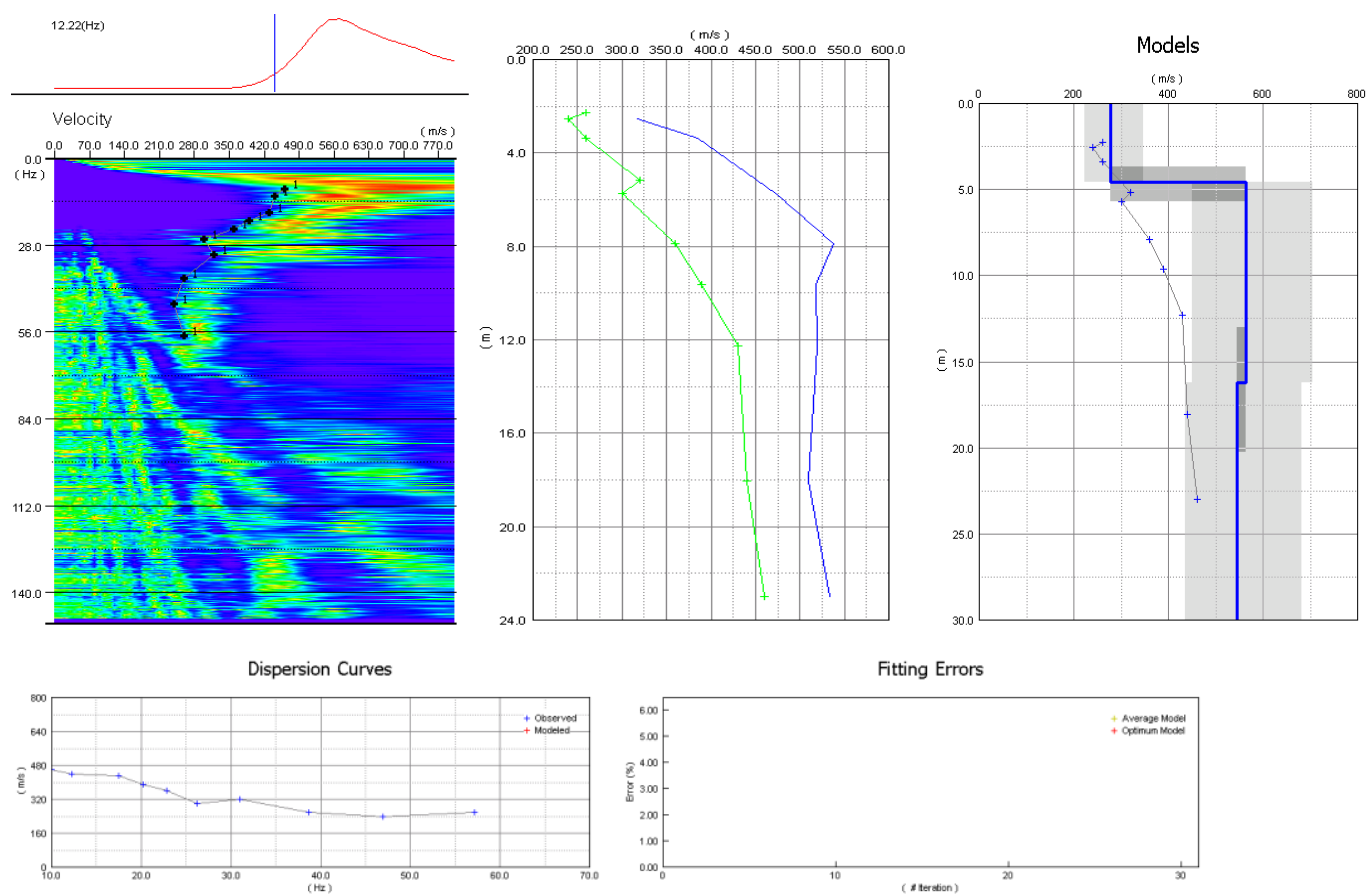

\section{Meter Survey}
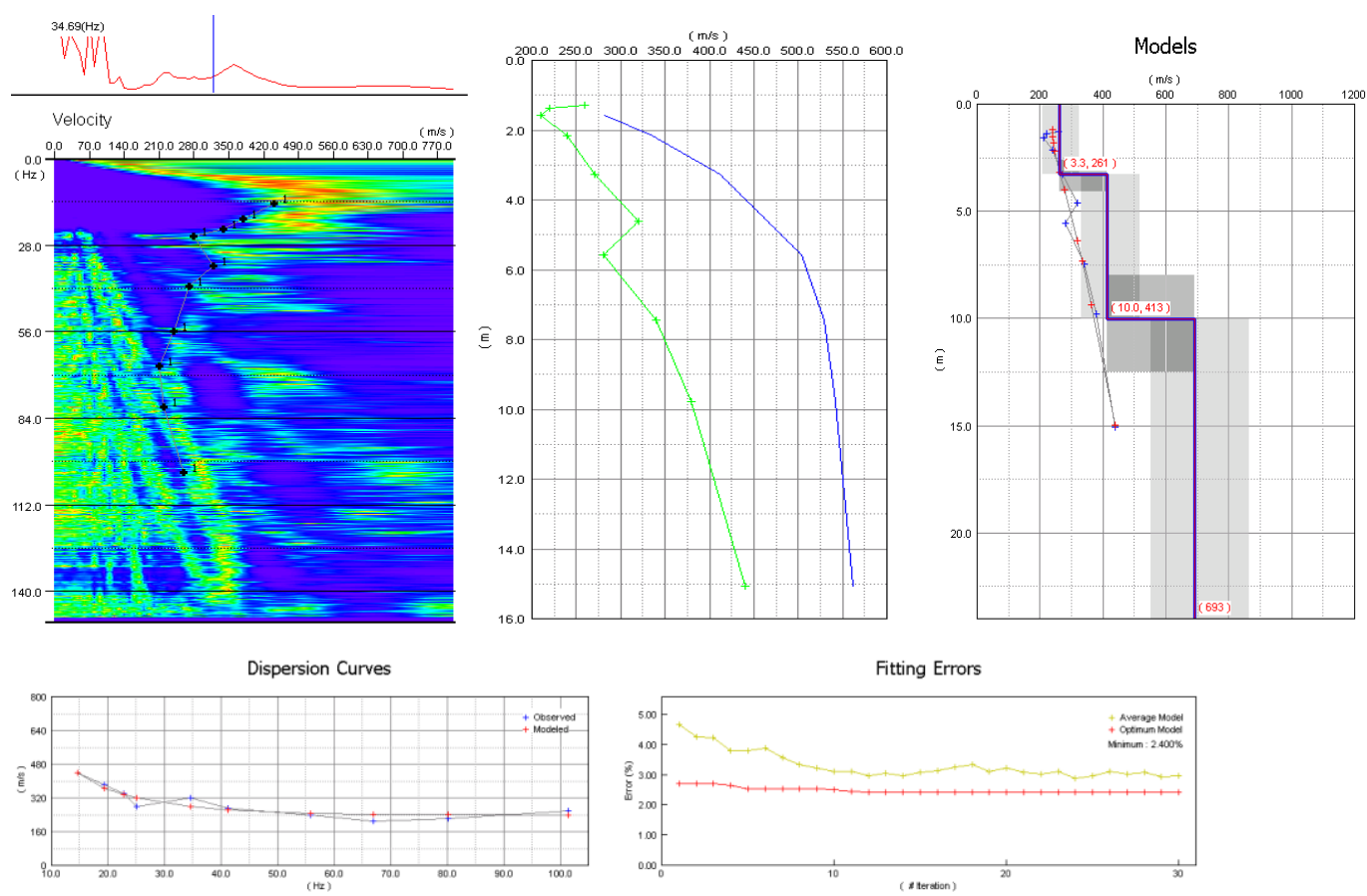


\section{Meter Survey}
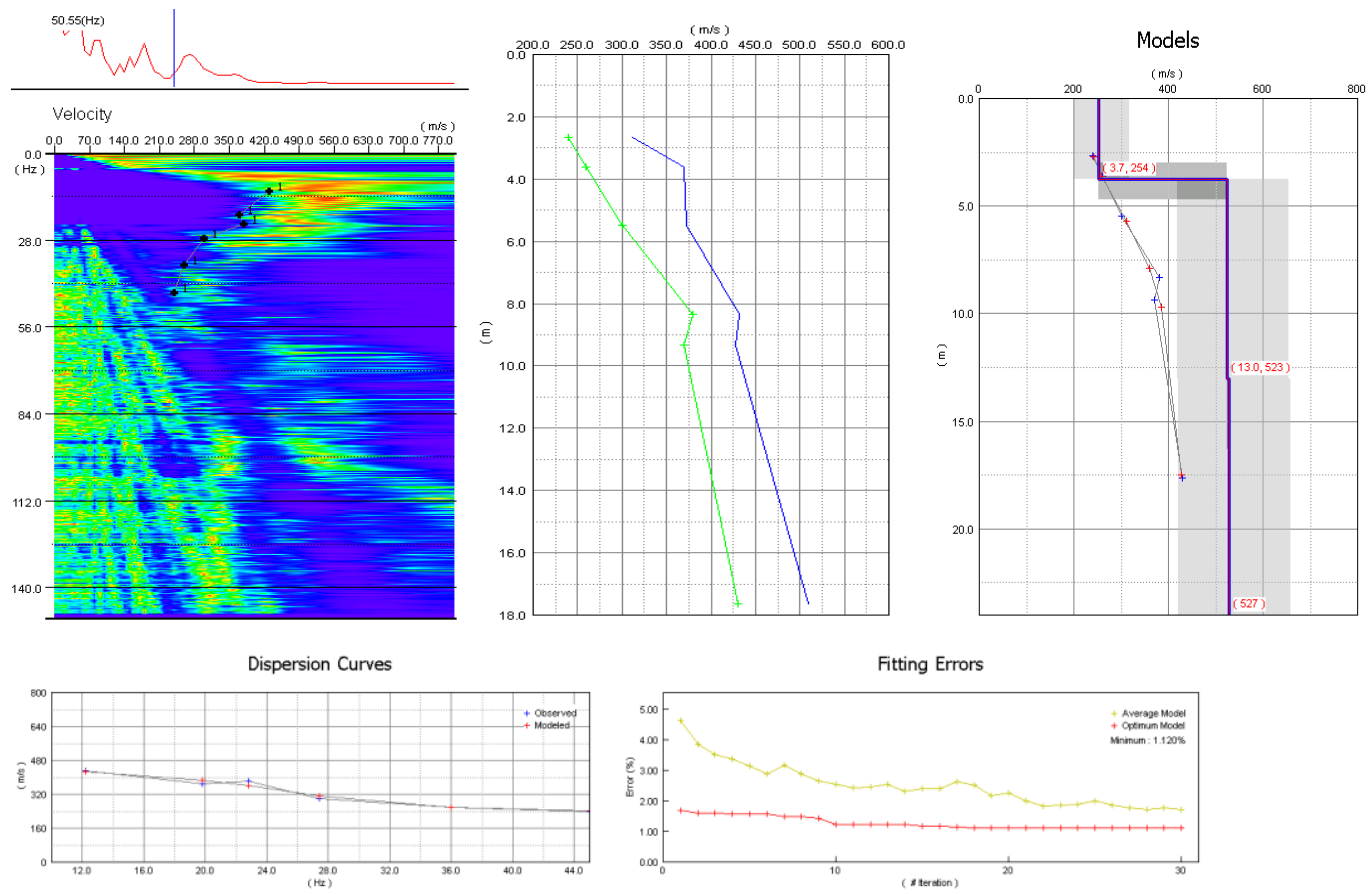

\section{Meter Survey}
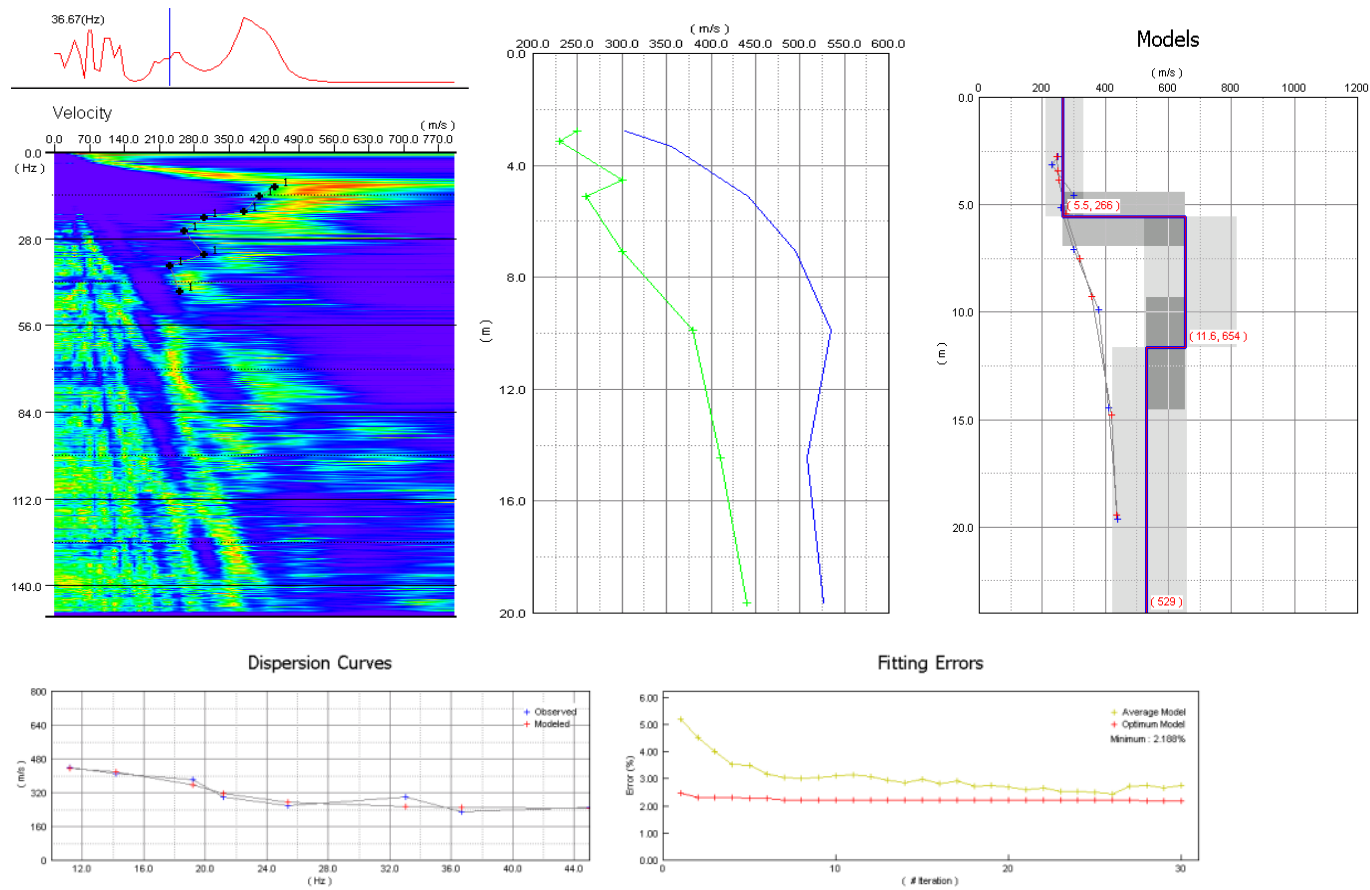


\section{Meter Survey}
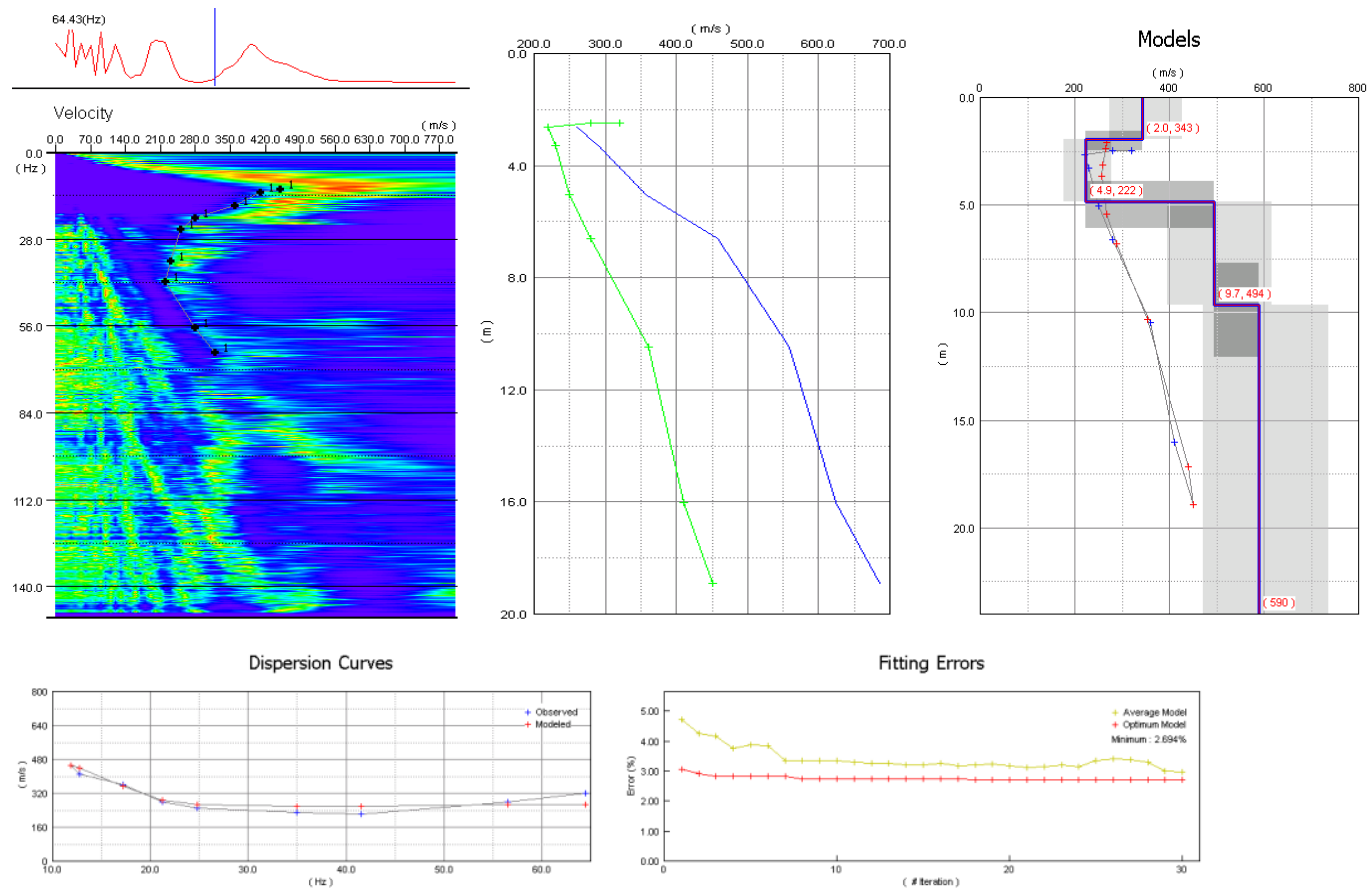

\section{Meter Survey}
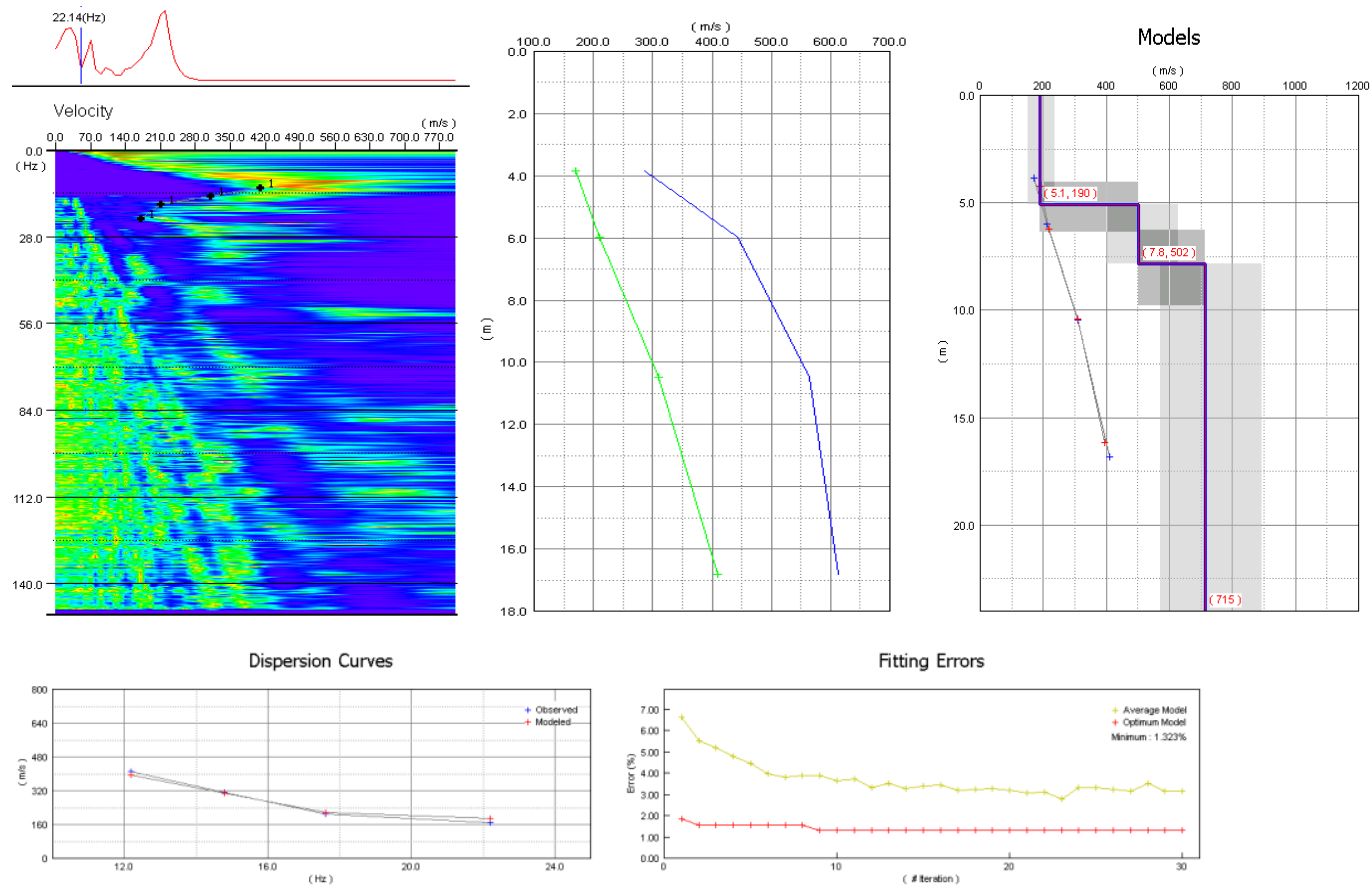


\section{Meter Survey}

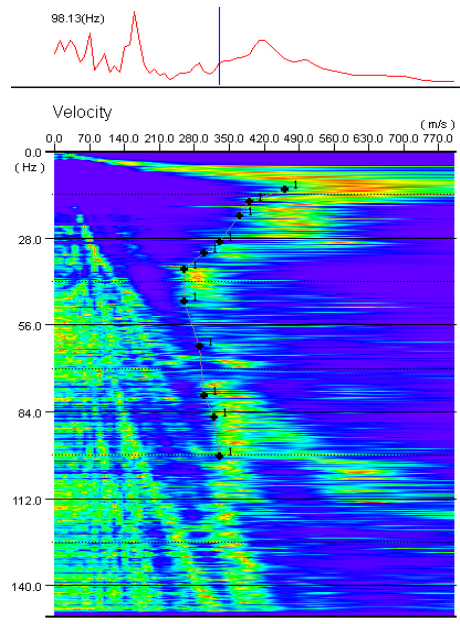

Dispersion Curves

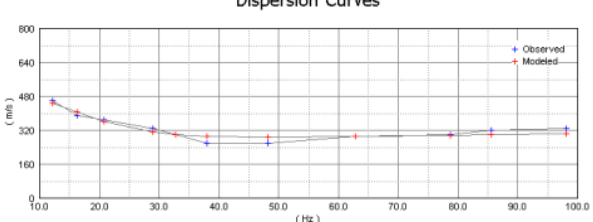

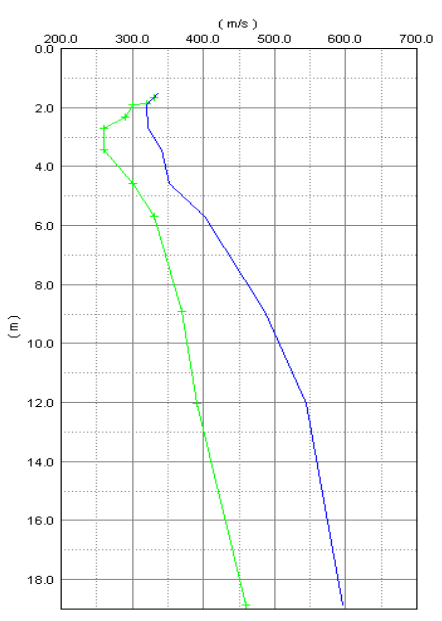

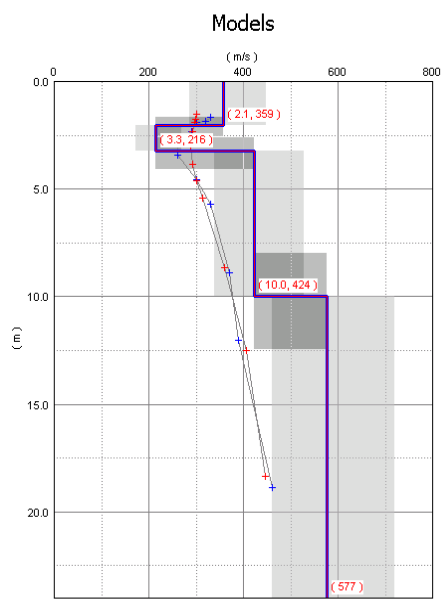

Fitting Errors

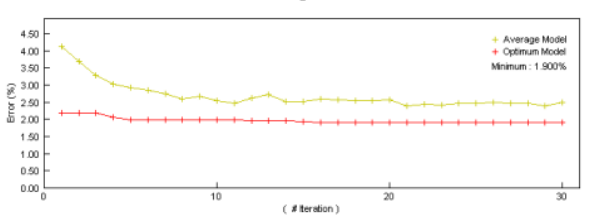

70 Meter Survey
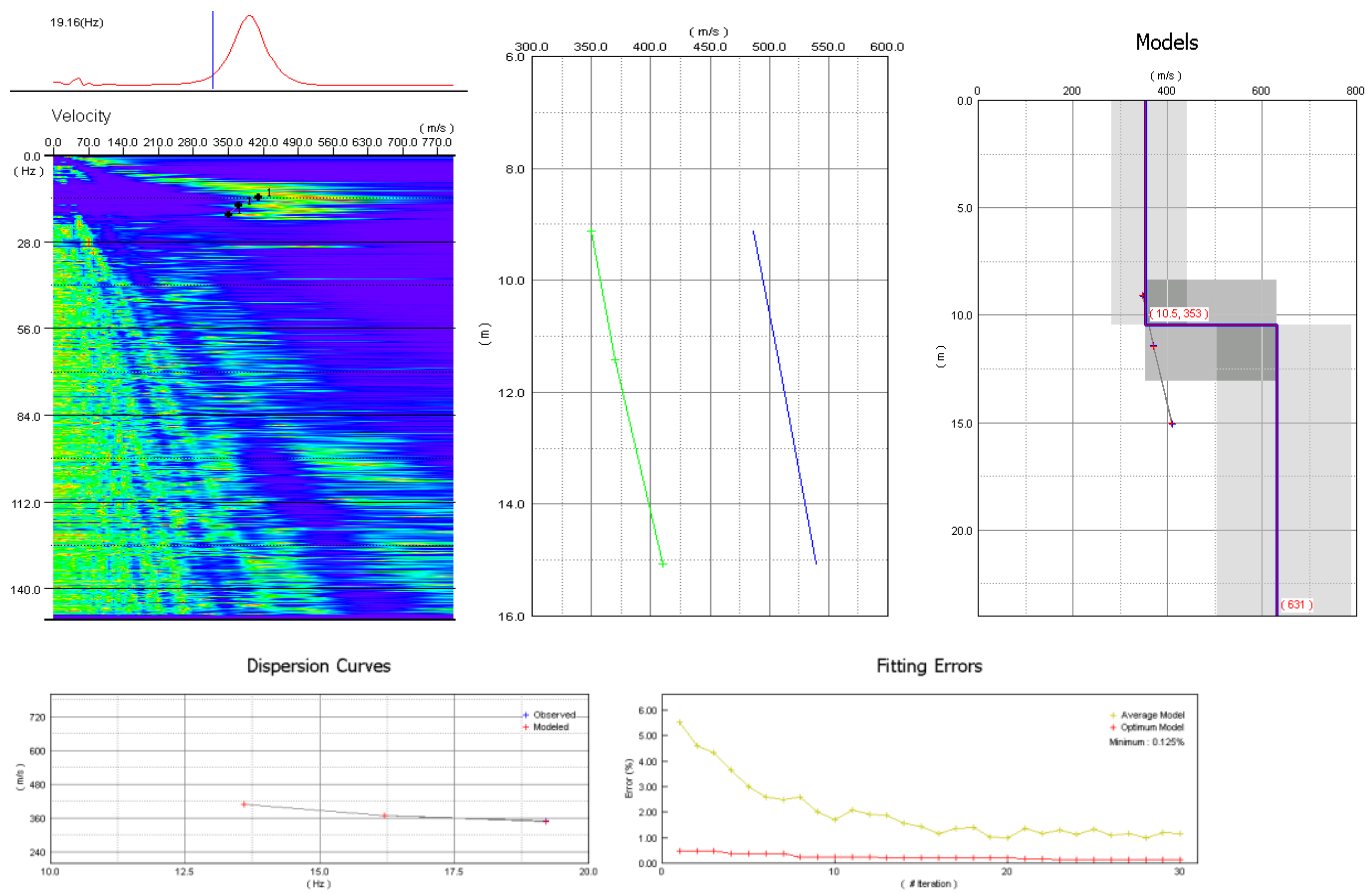
75 Meter Survey
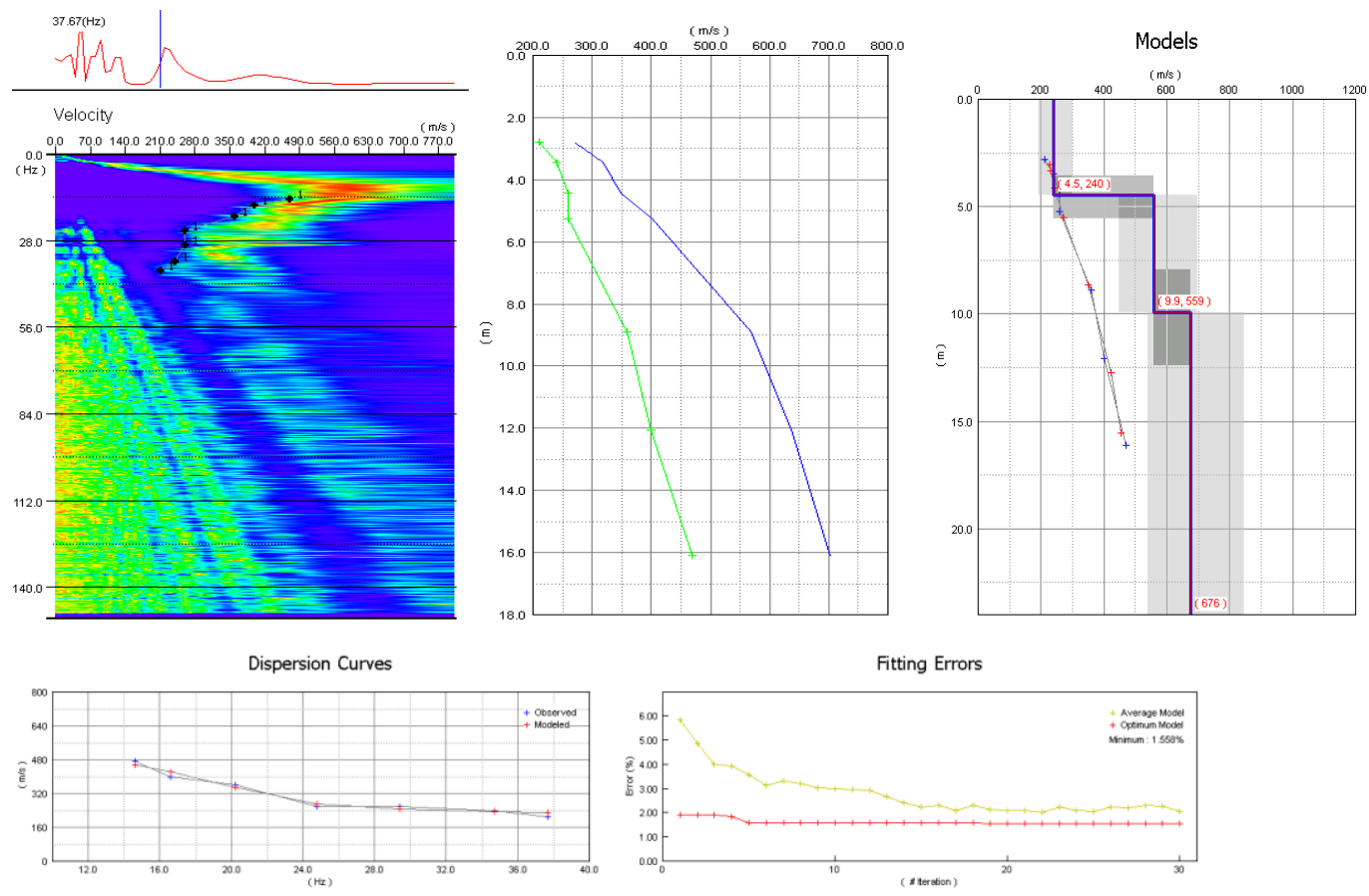

\section{Meter Survey}
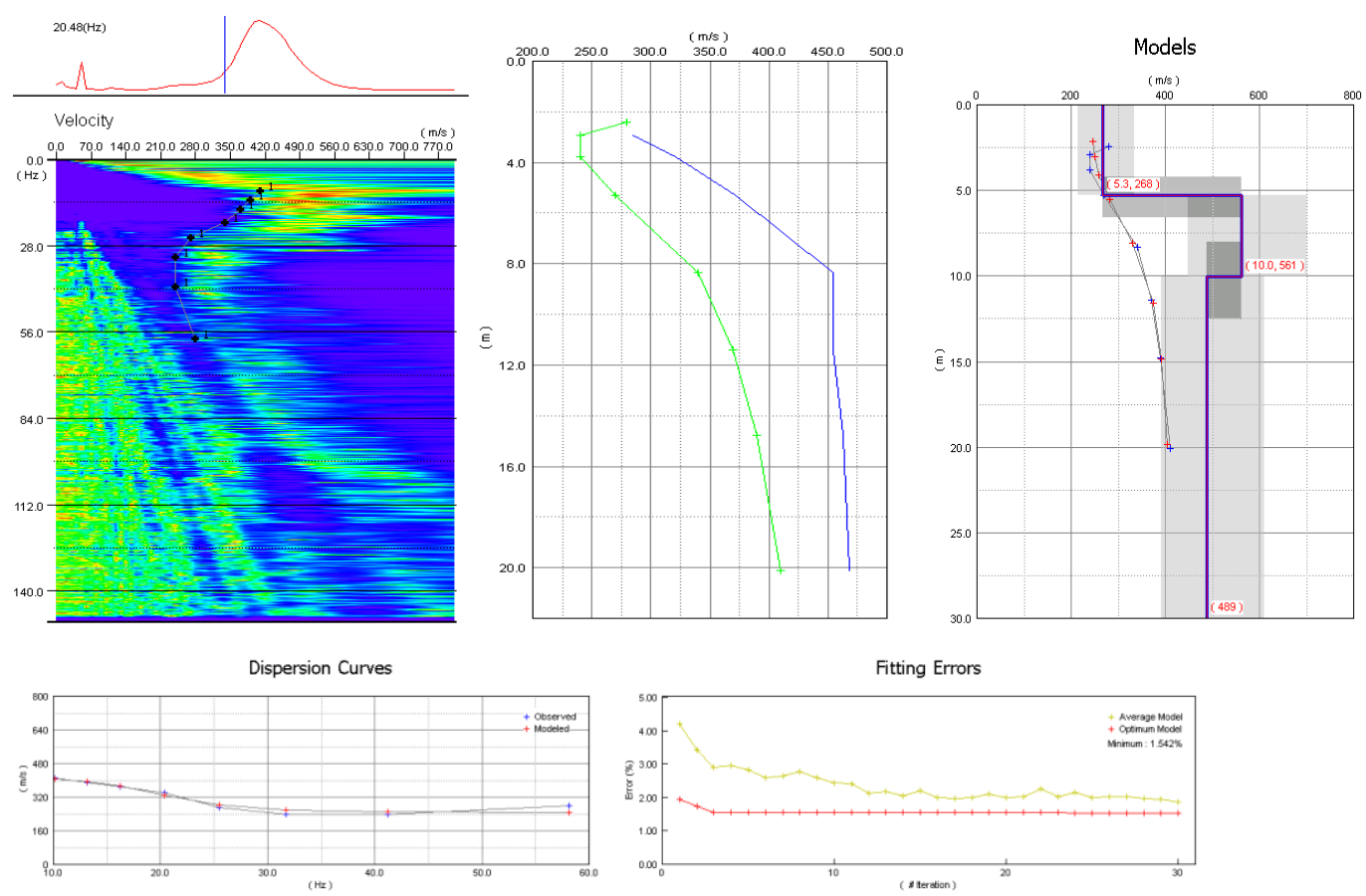


\section{Meter Survey}
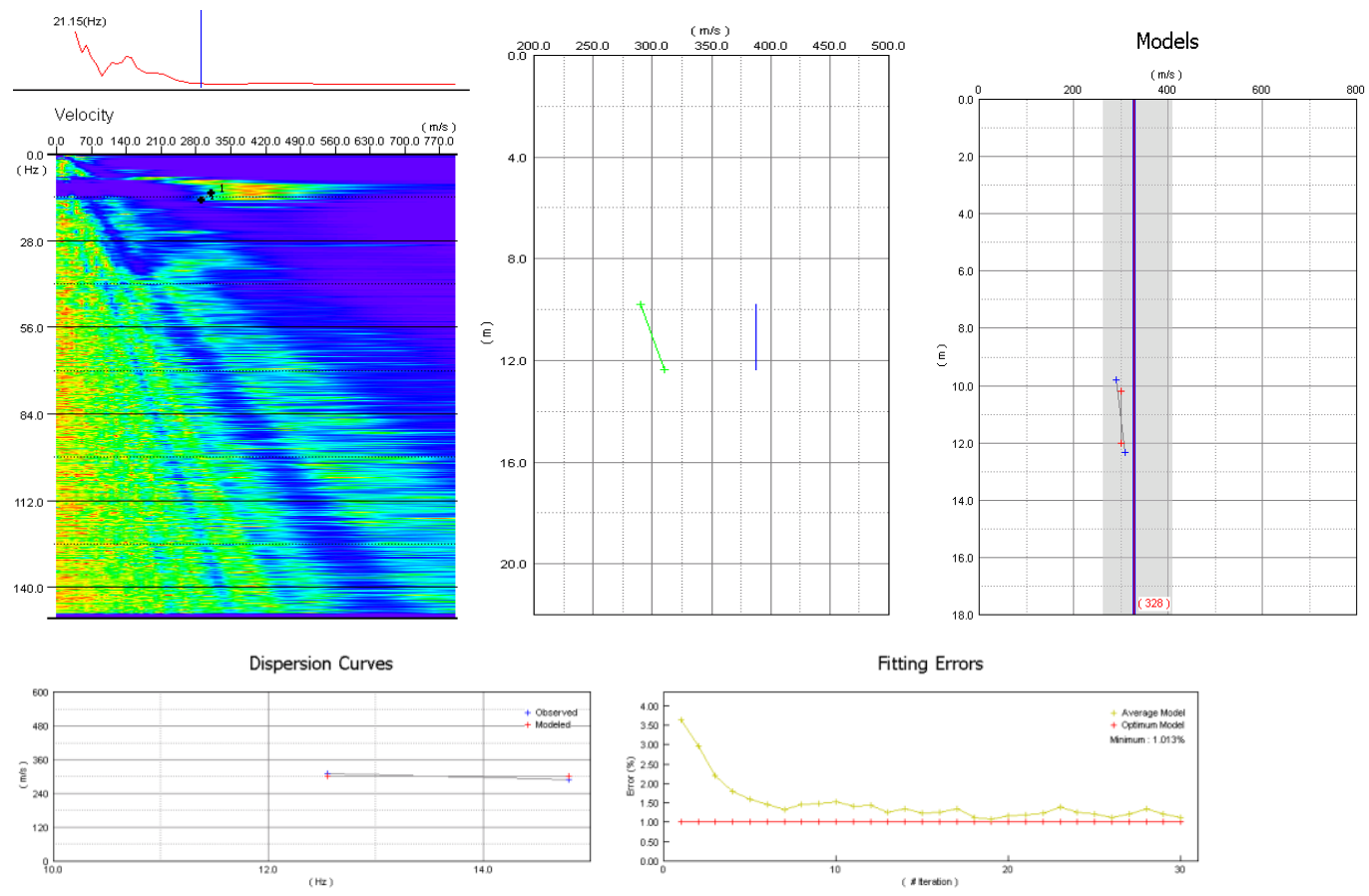

\section{Meter Survey}
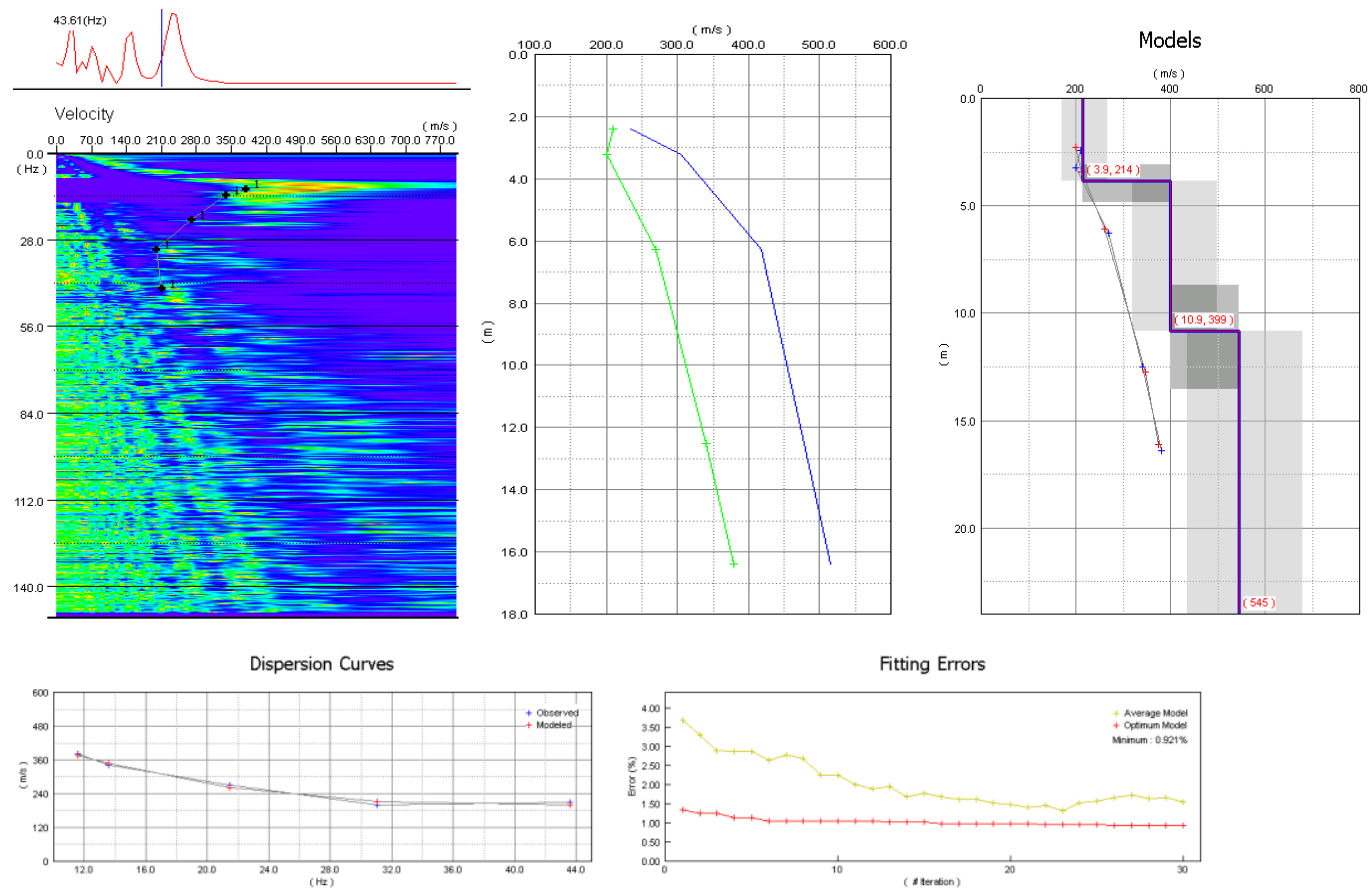


\section{Meter Survey}
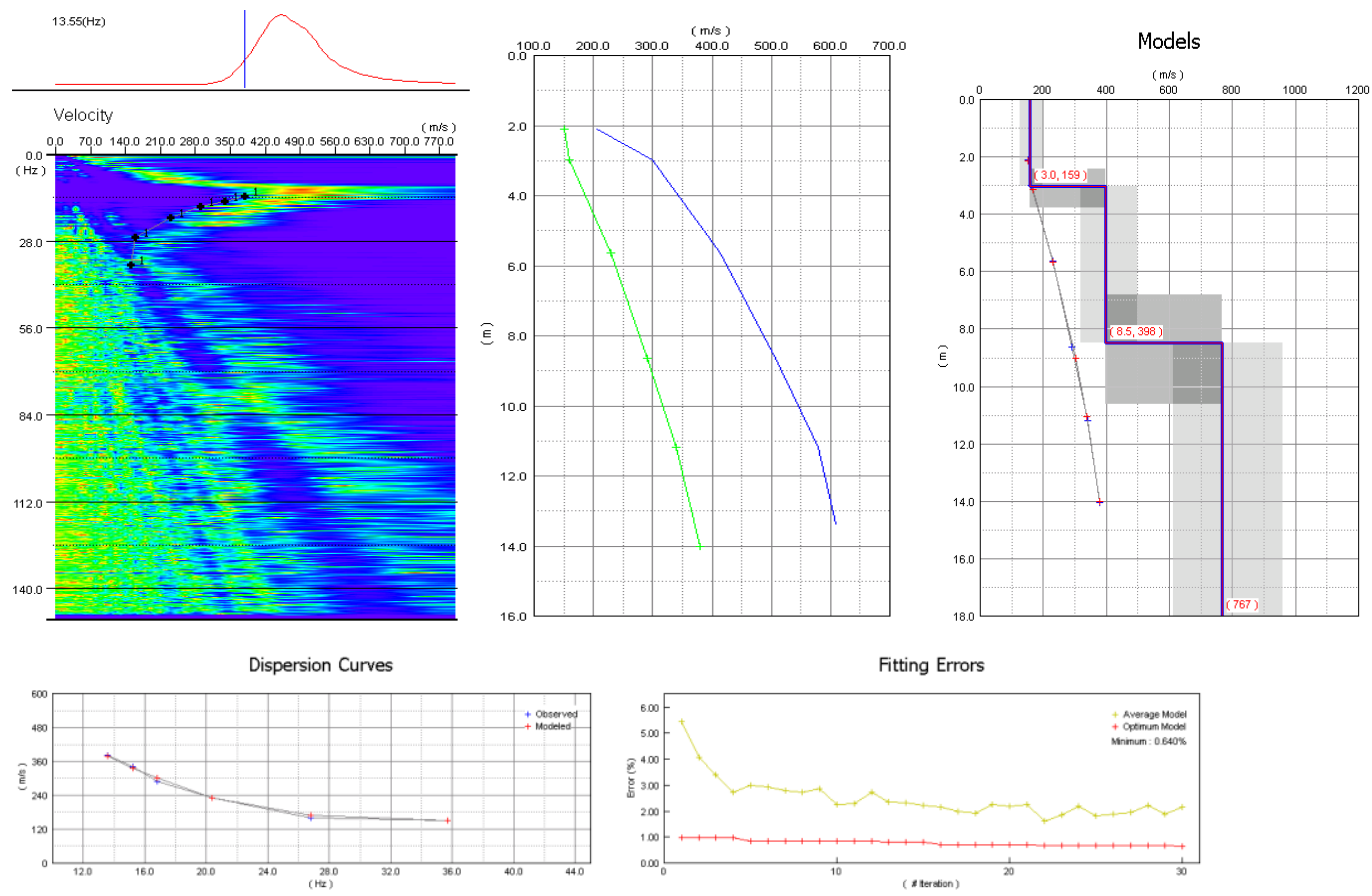

100 Meter Survey
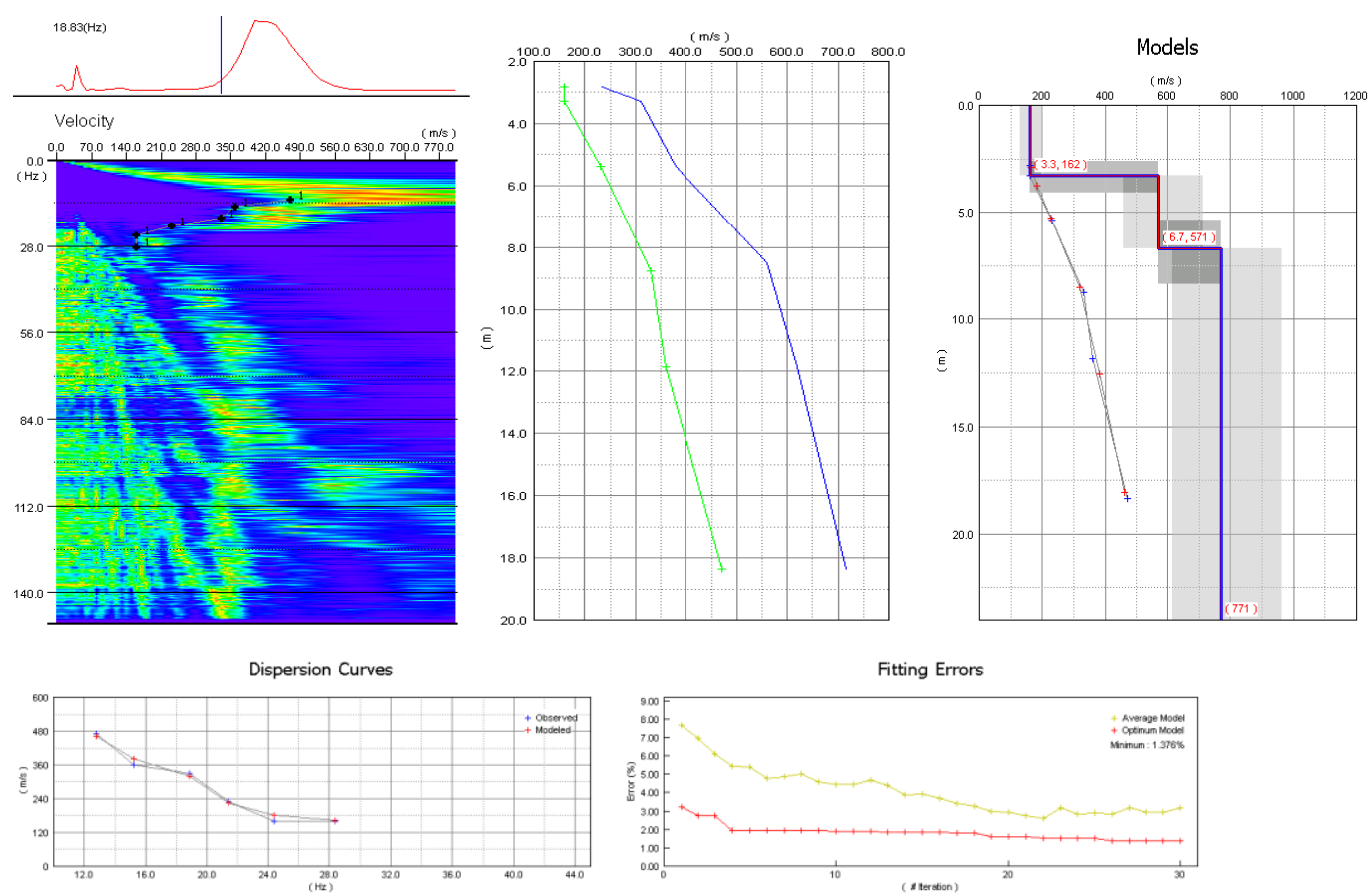
105 Meter Survey
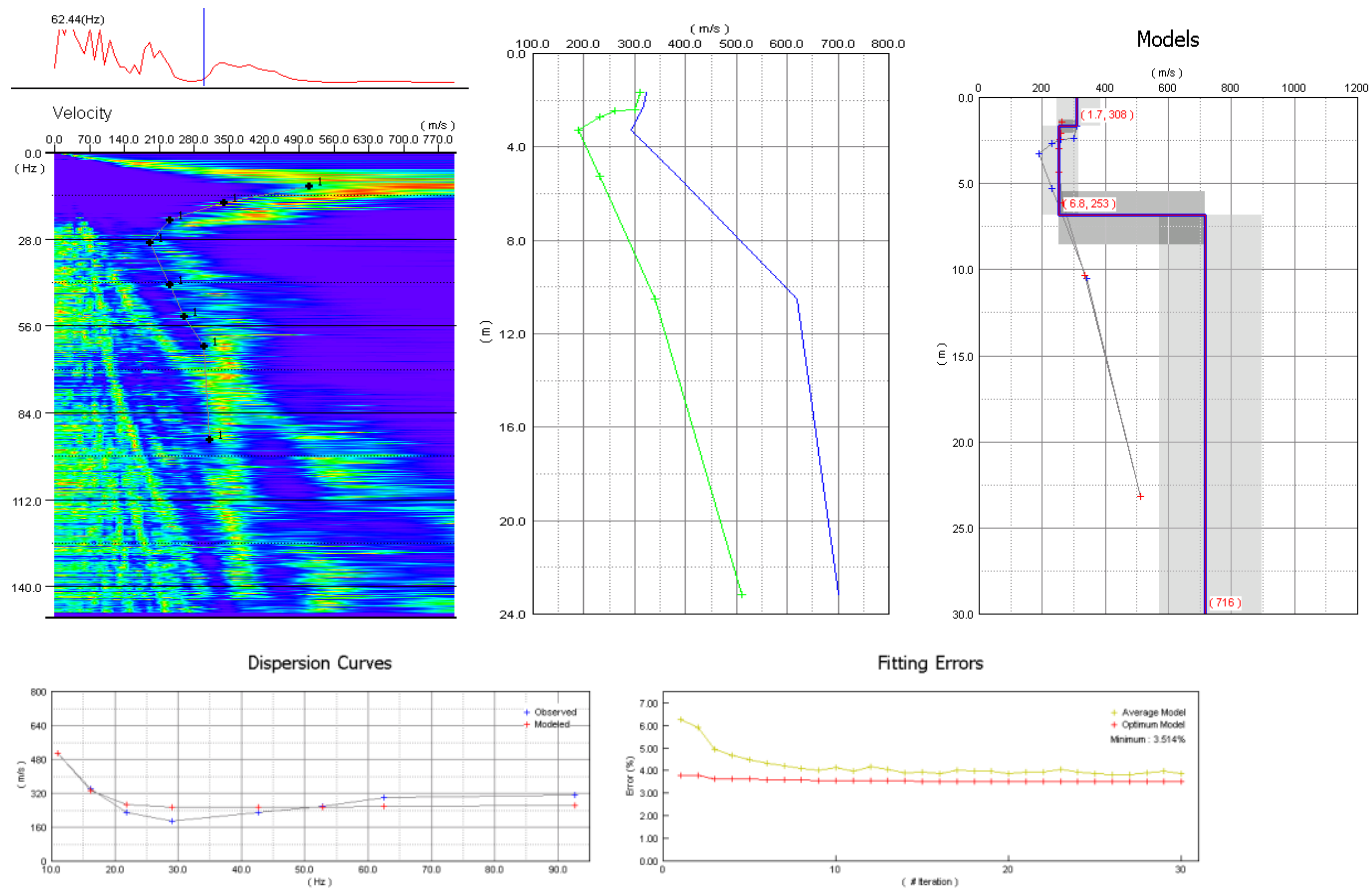

\section{Meter Survey}
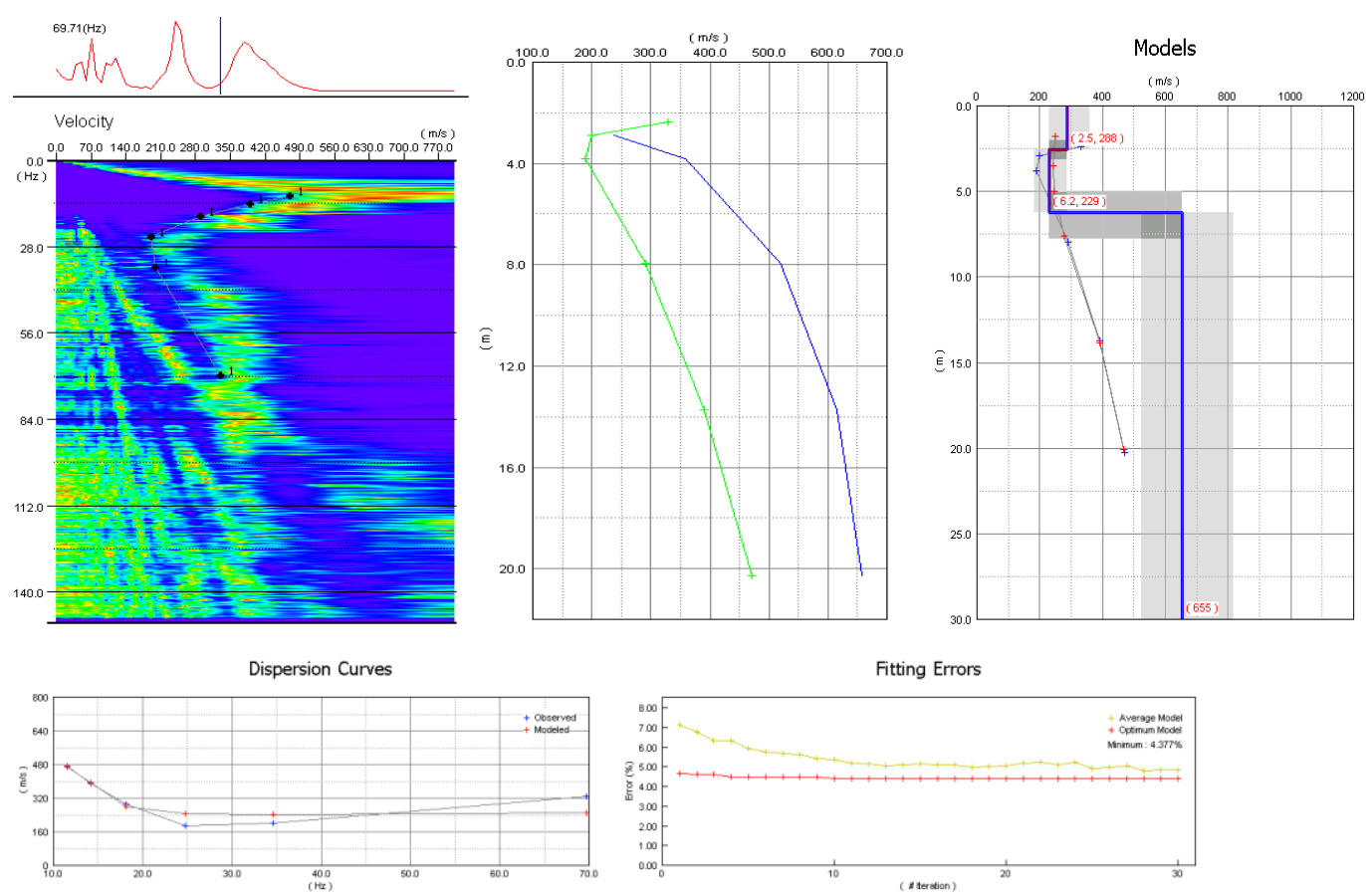


\section{Meter Survey}
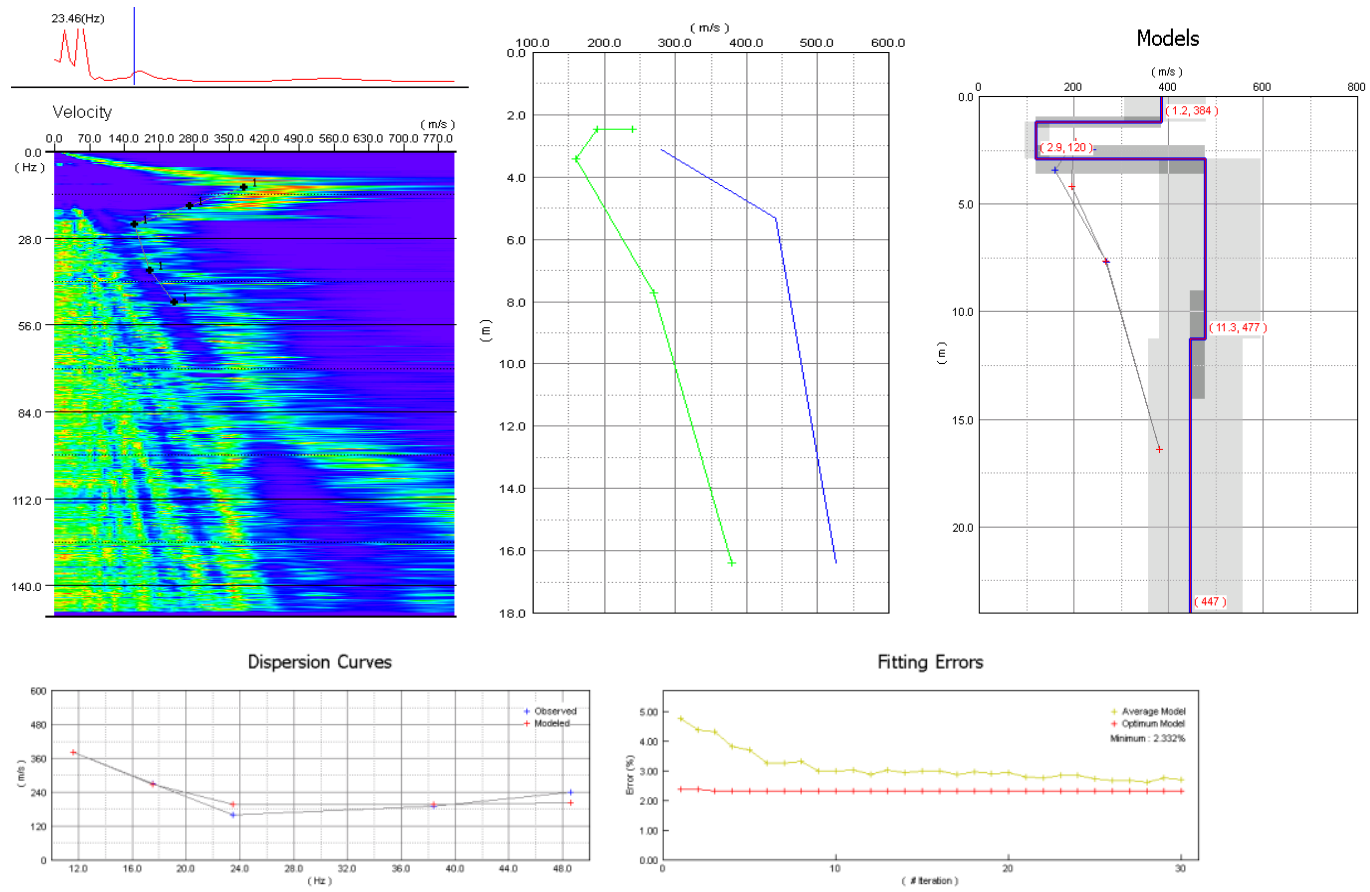

\section{Meter Survey}
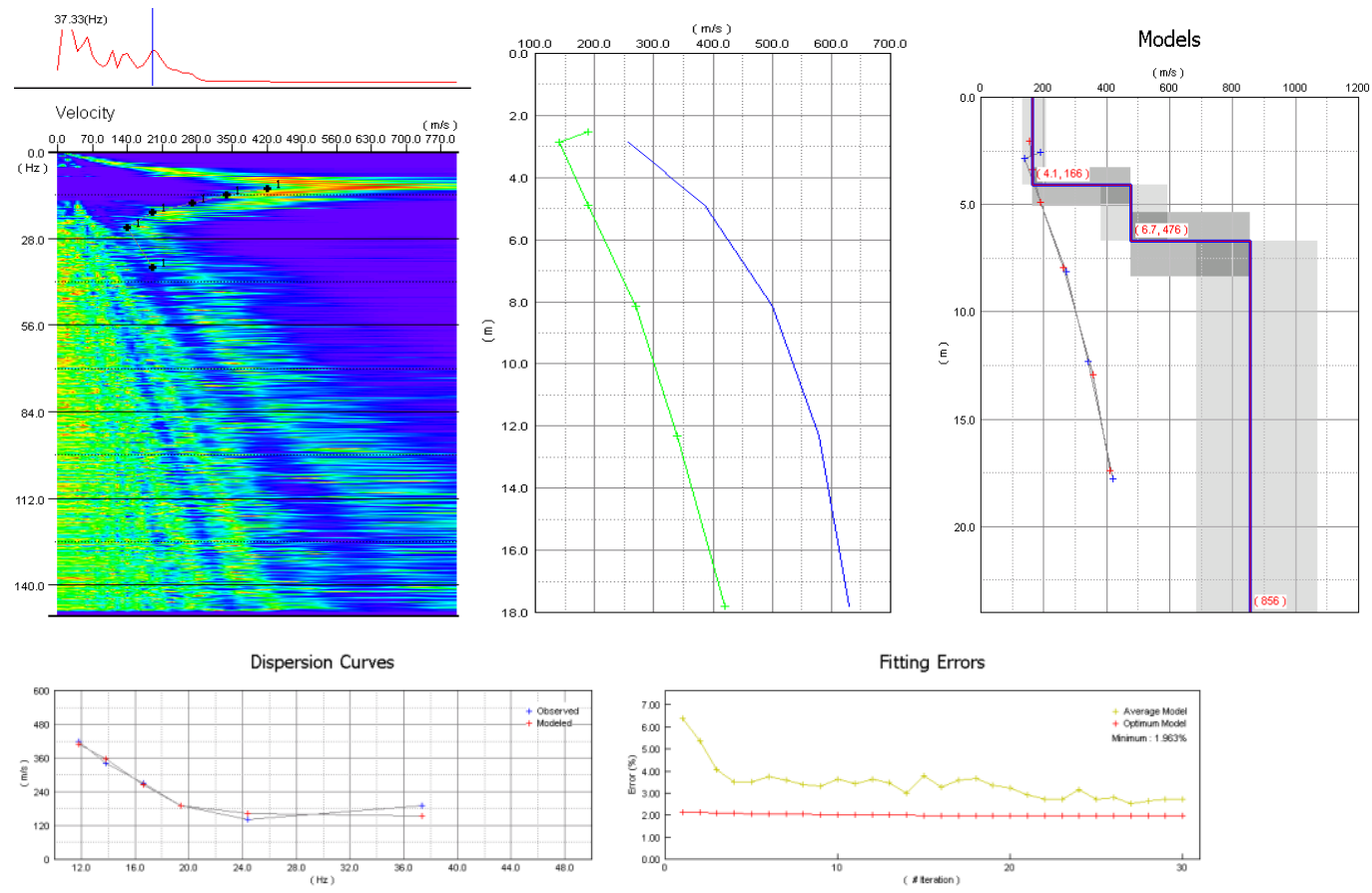
125 Meter Survey
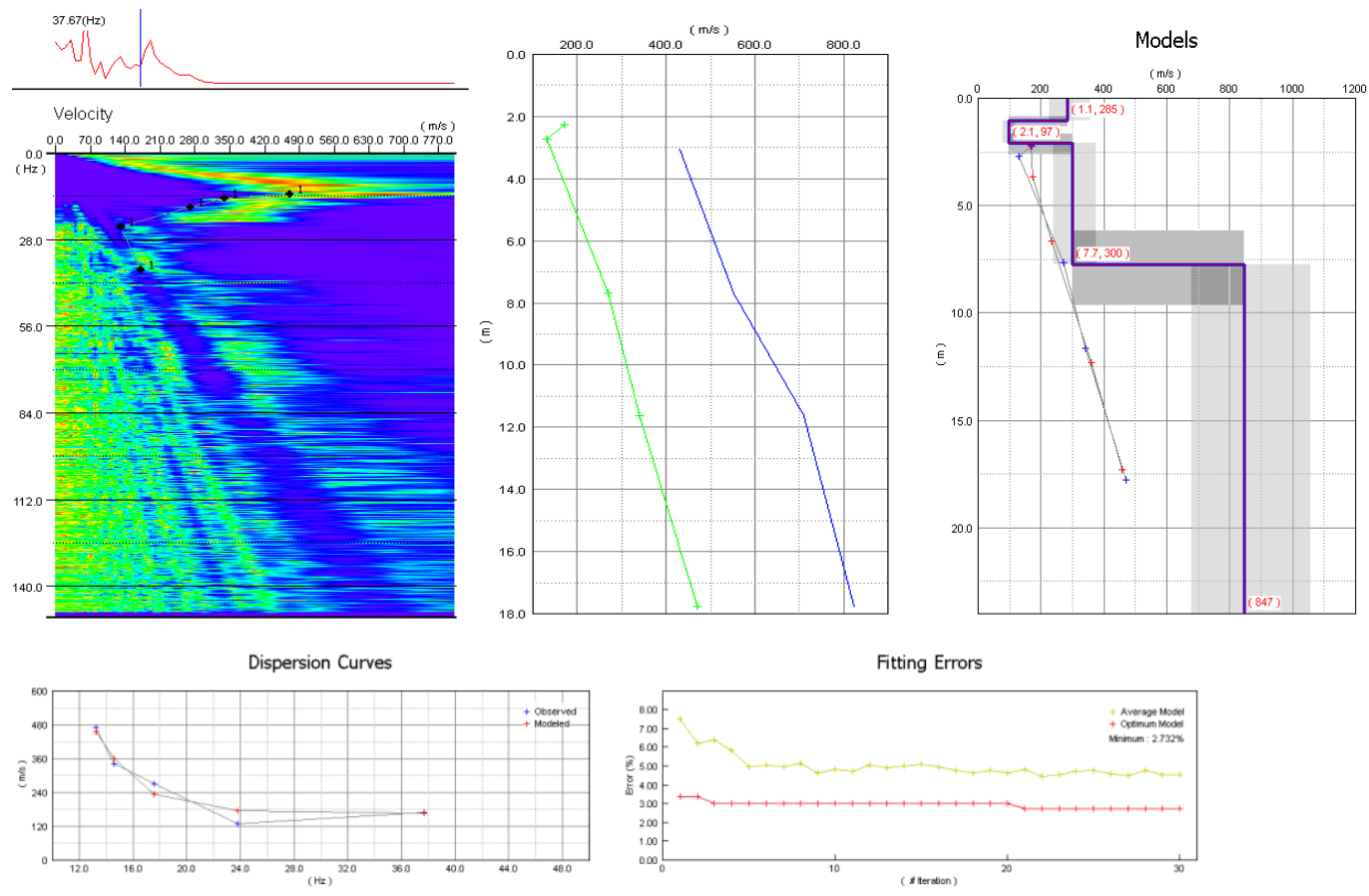

\section{Meter Survey}
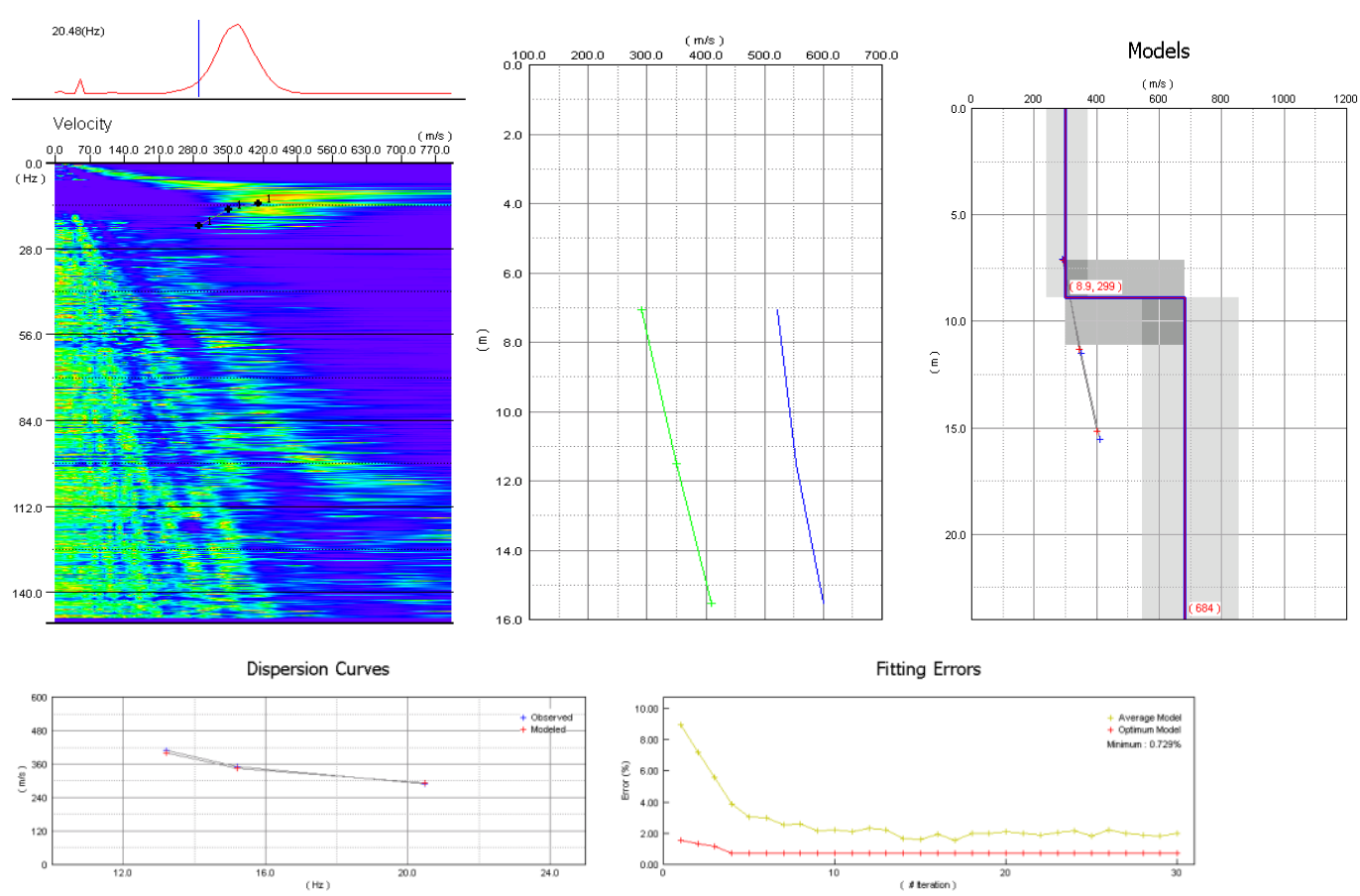


\section{Meter Survey}
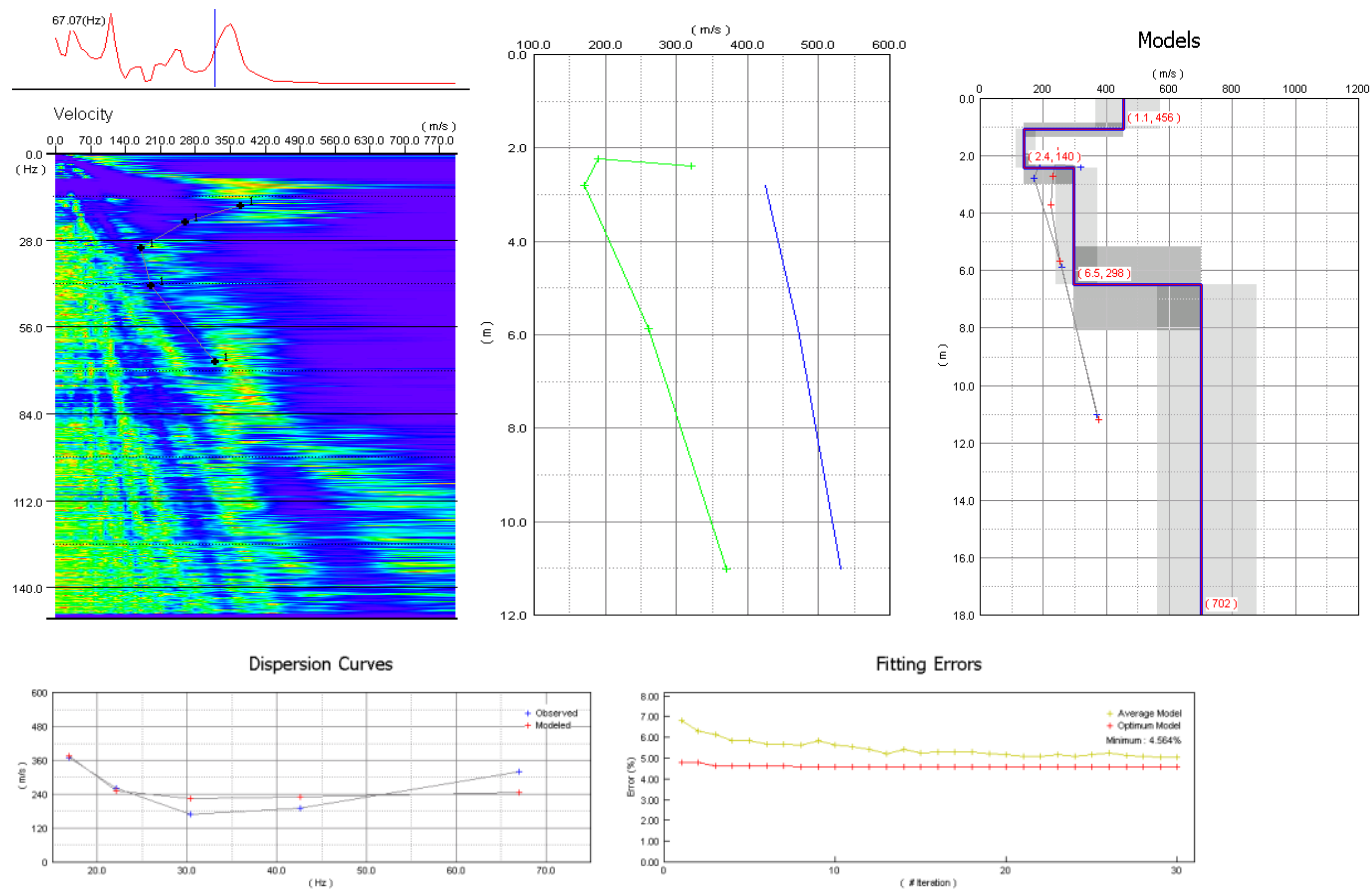

\section{Meter Survey}
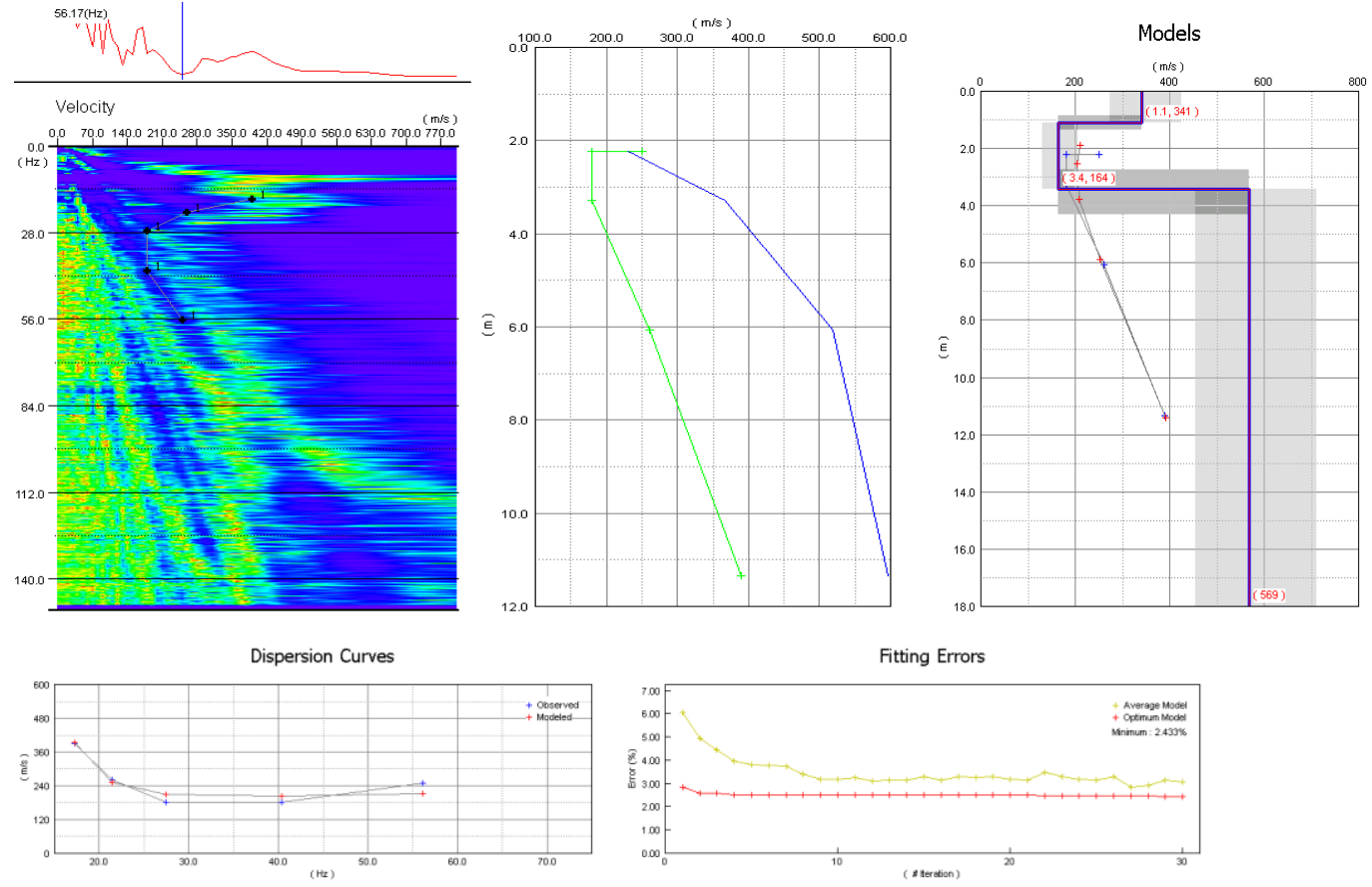
145 Meter Survey
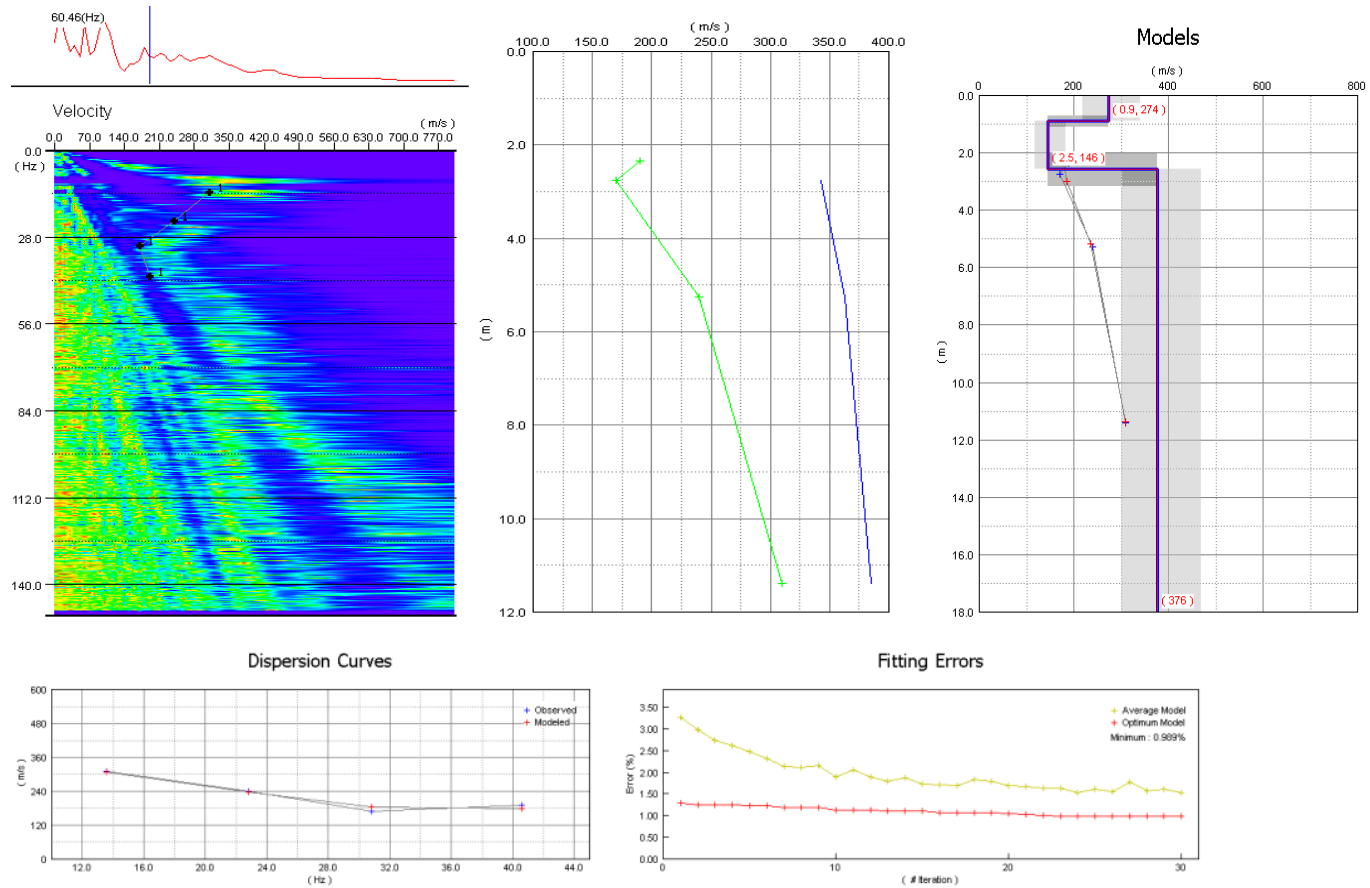

150 Meter Survey
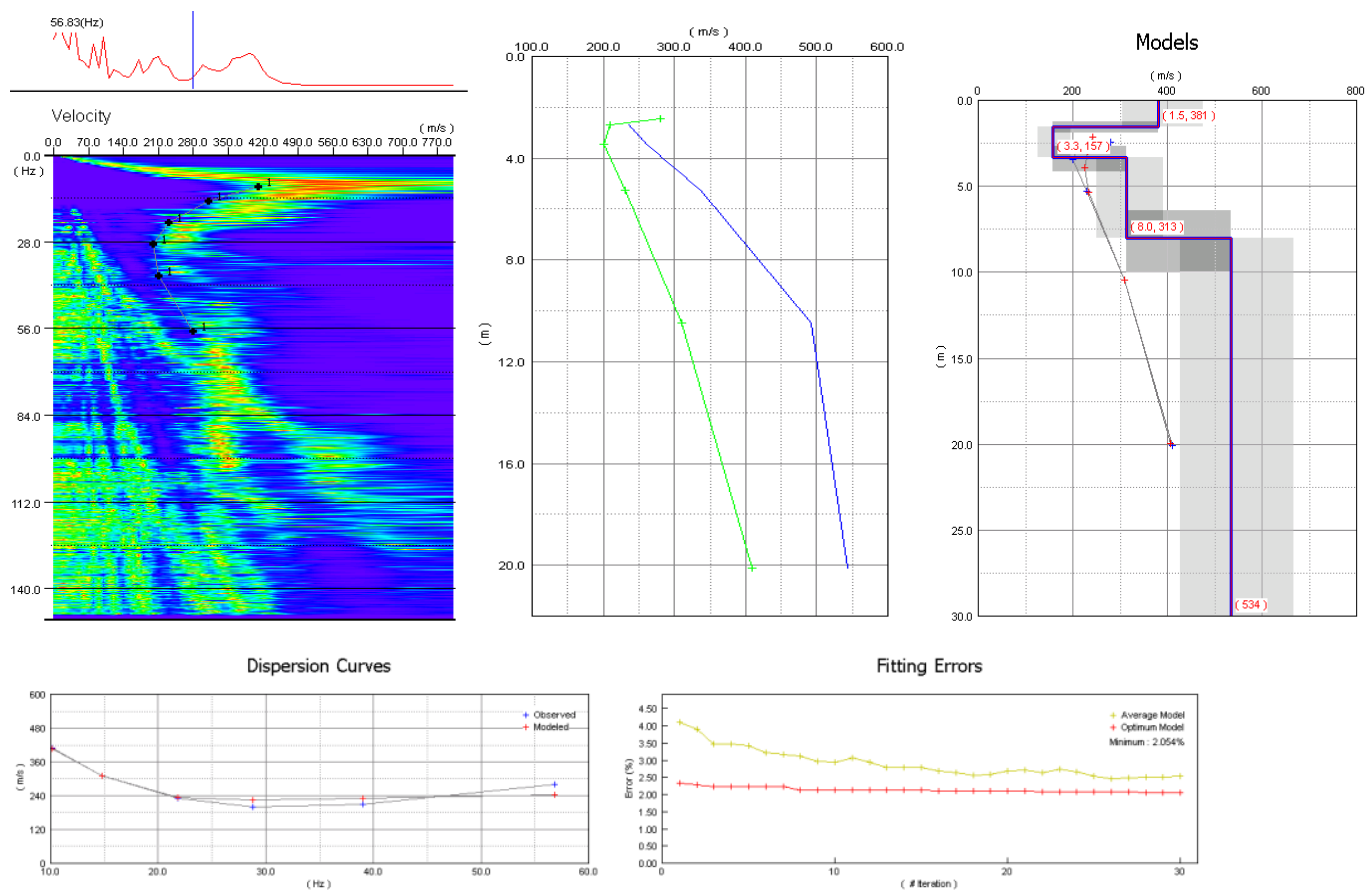
155 Meter Survey
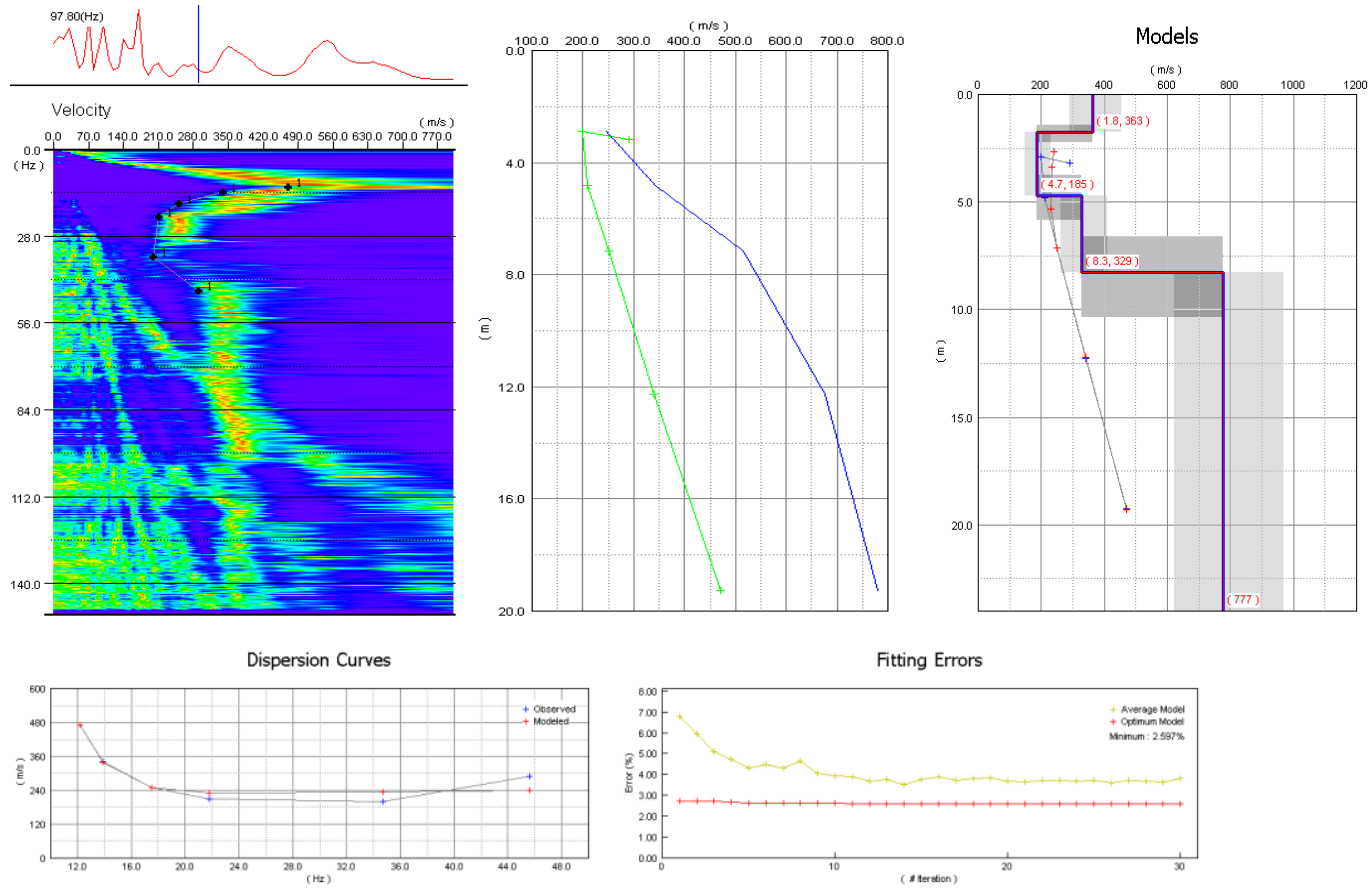

160 Meter Survey
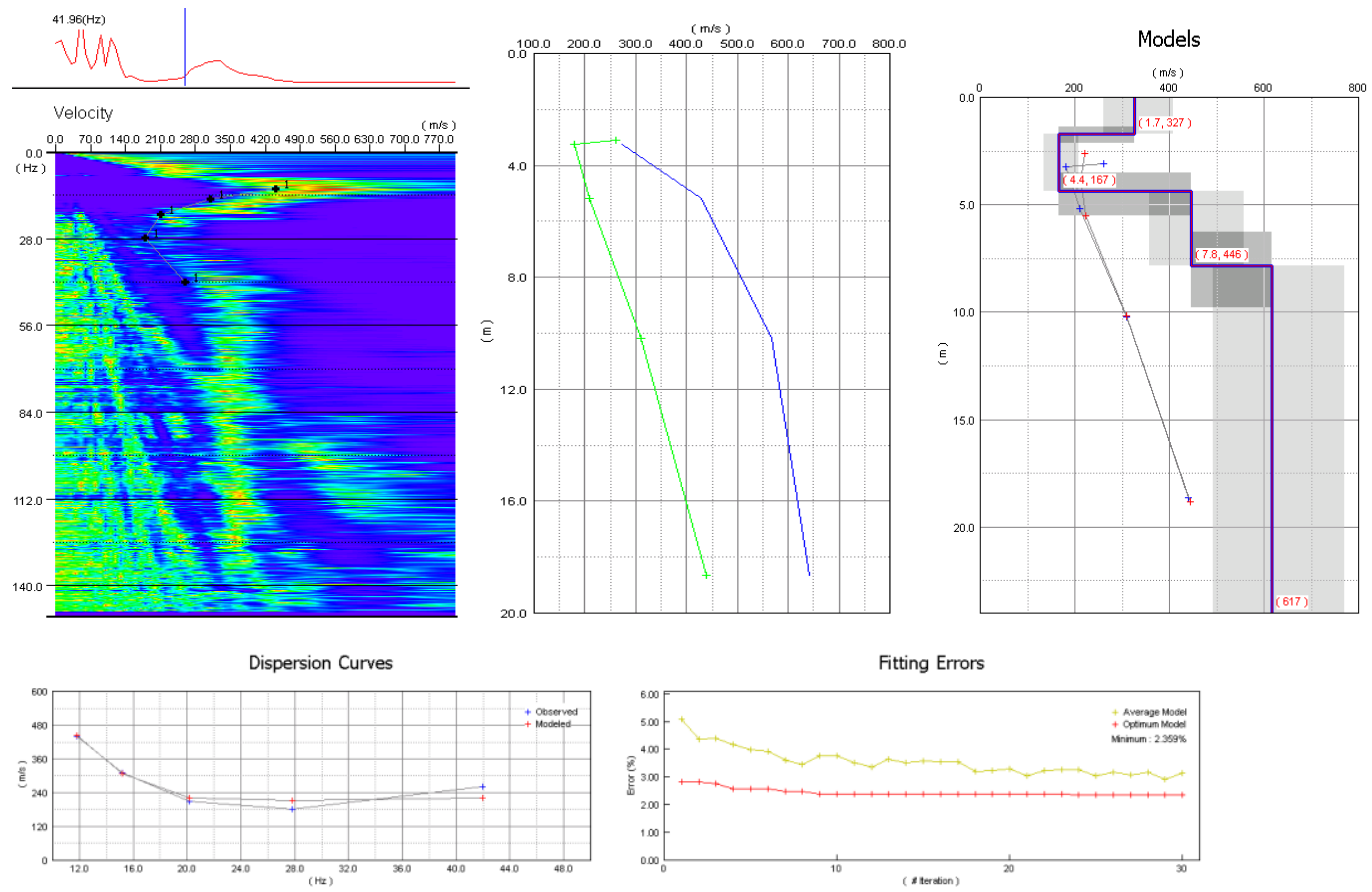
165 Meter Survey
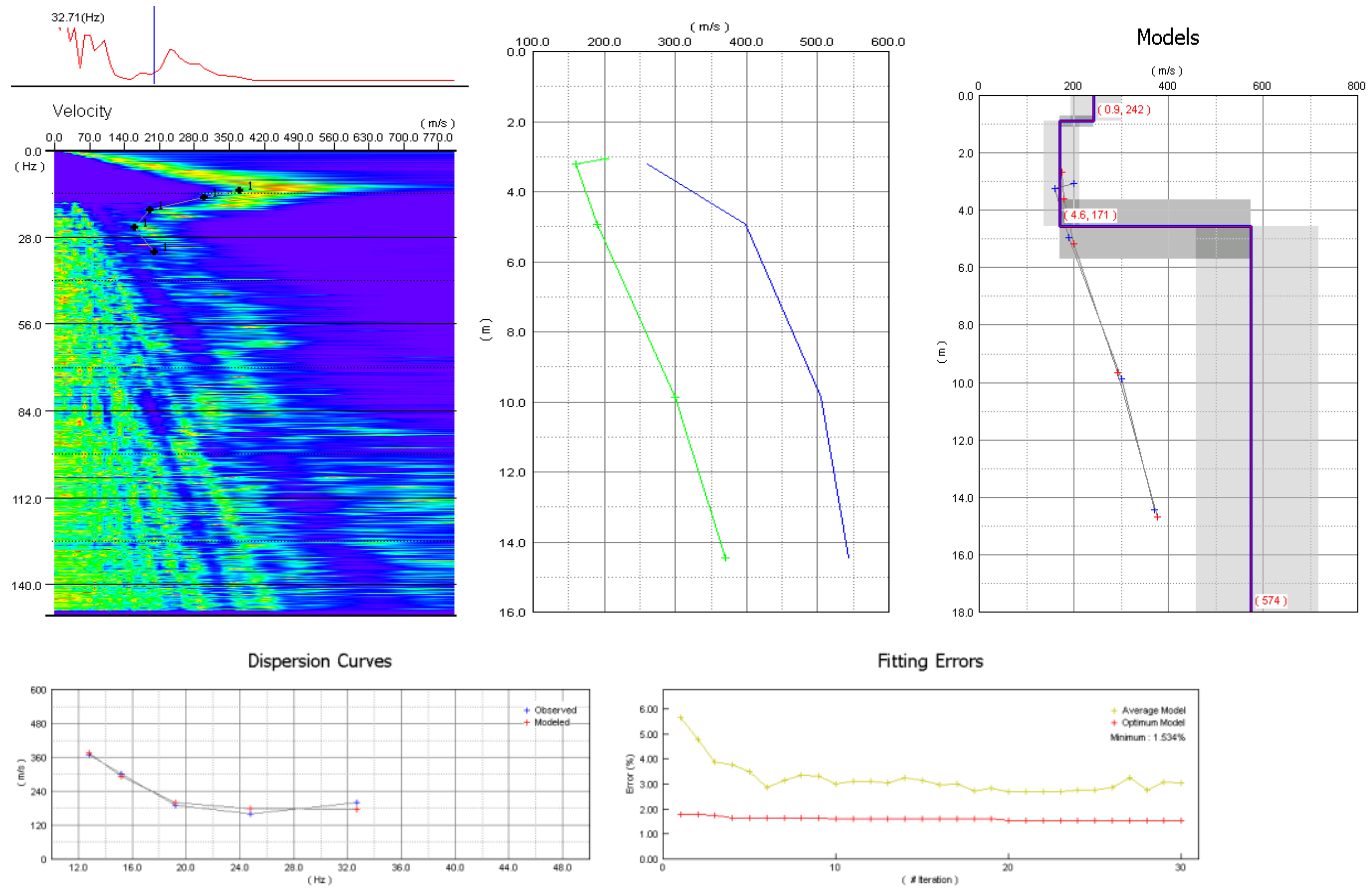

170 Meter Survey
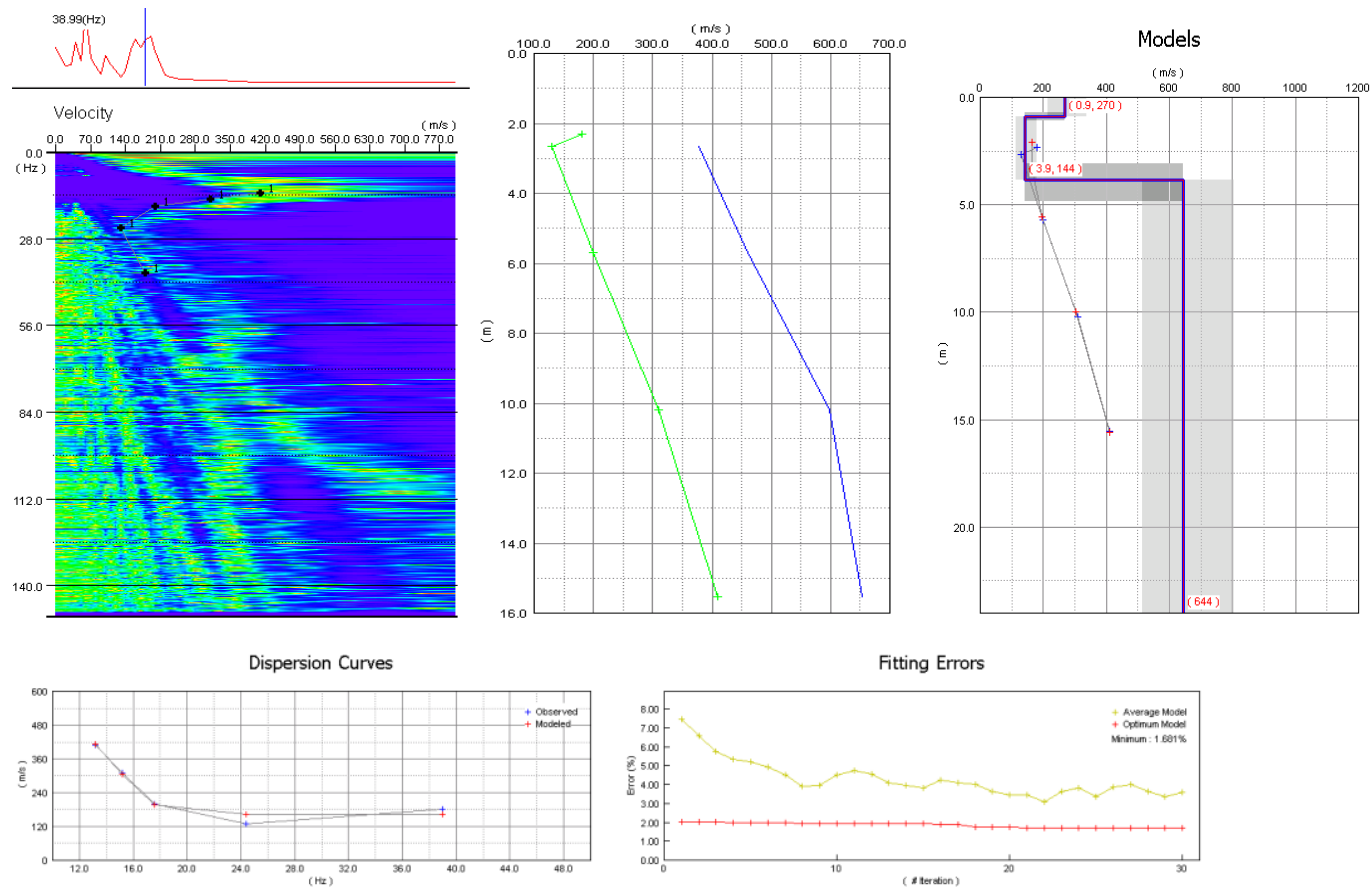
175 Meter Survey
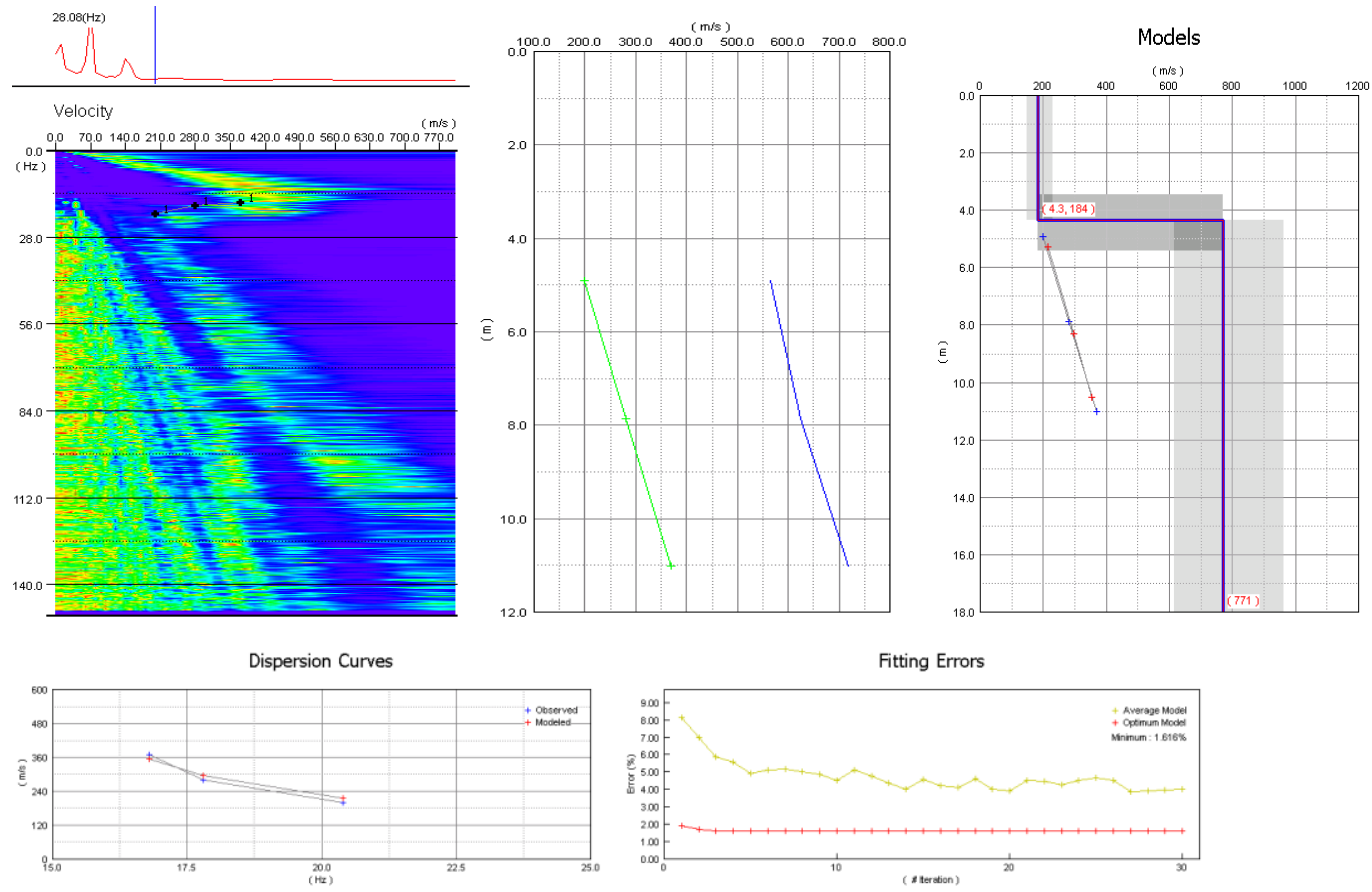

\section{Meter Survey}
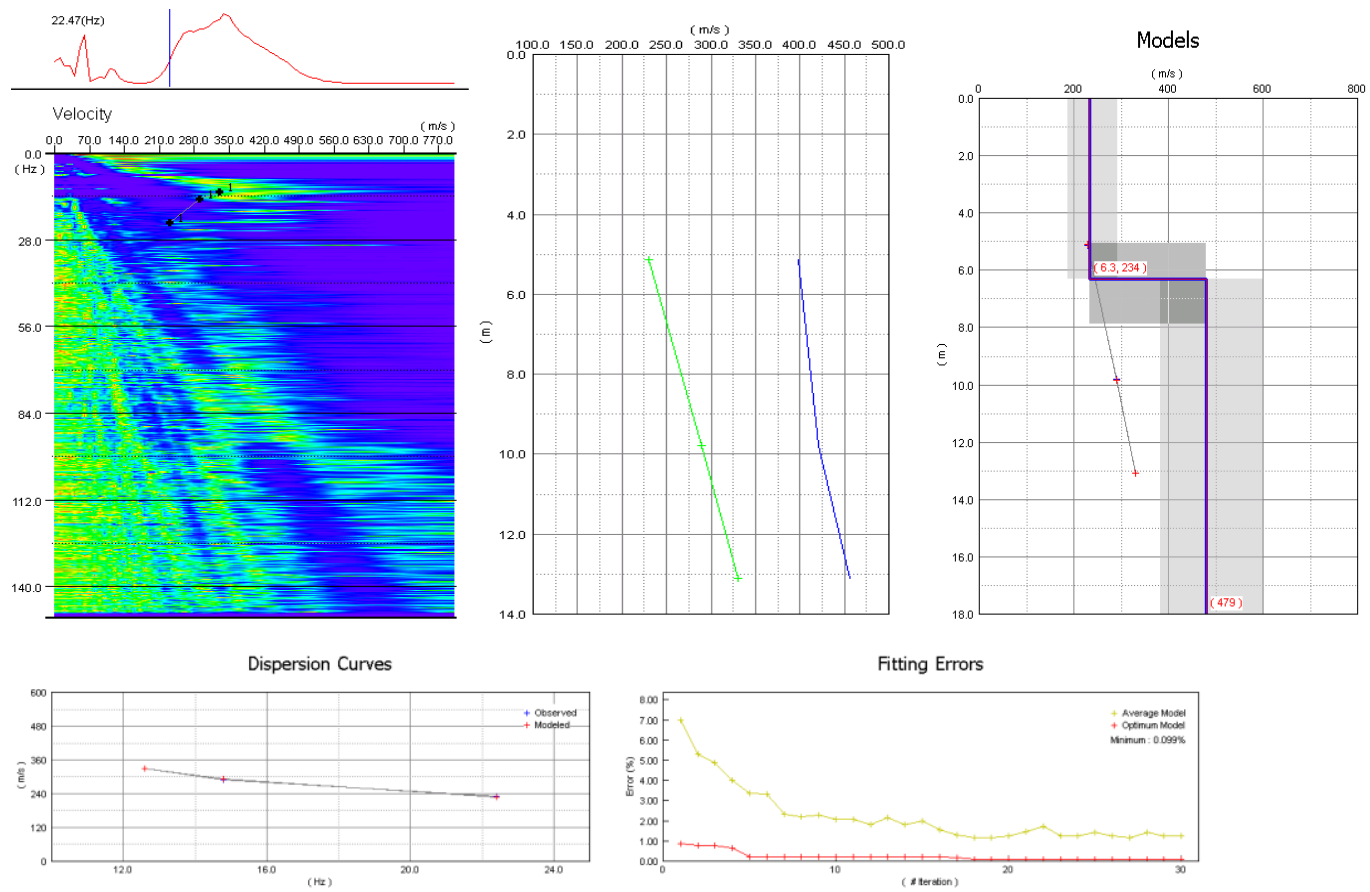


\section{Meter Survey}

* No $V_{R}$ dispersion curve available.
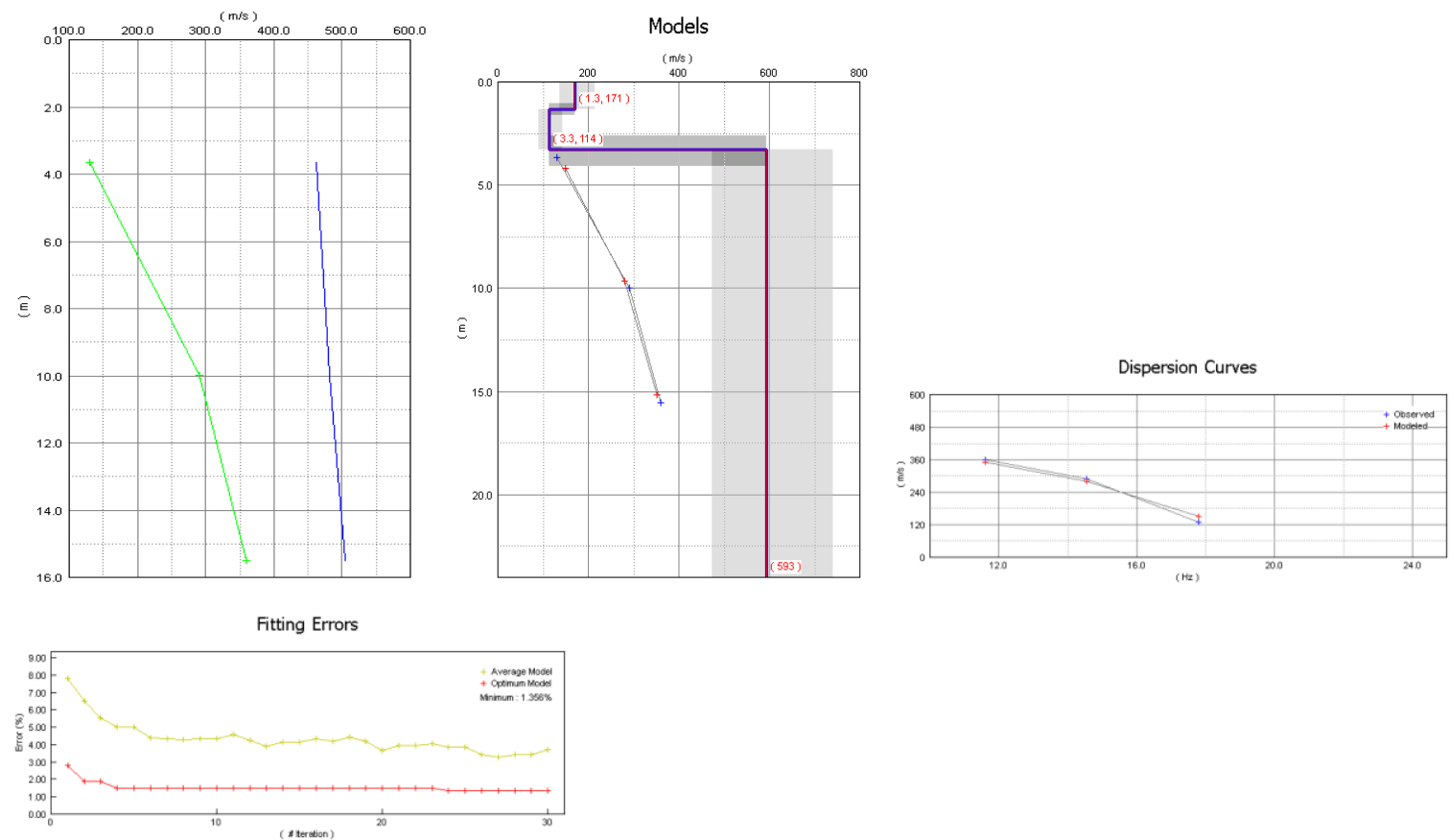

190 Meter Survey
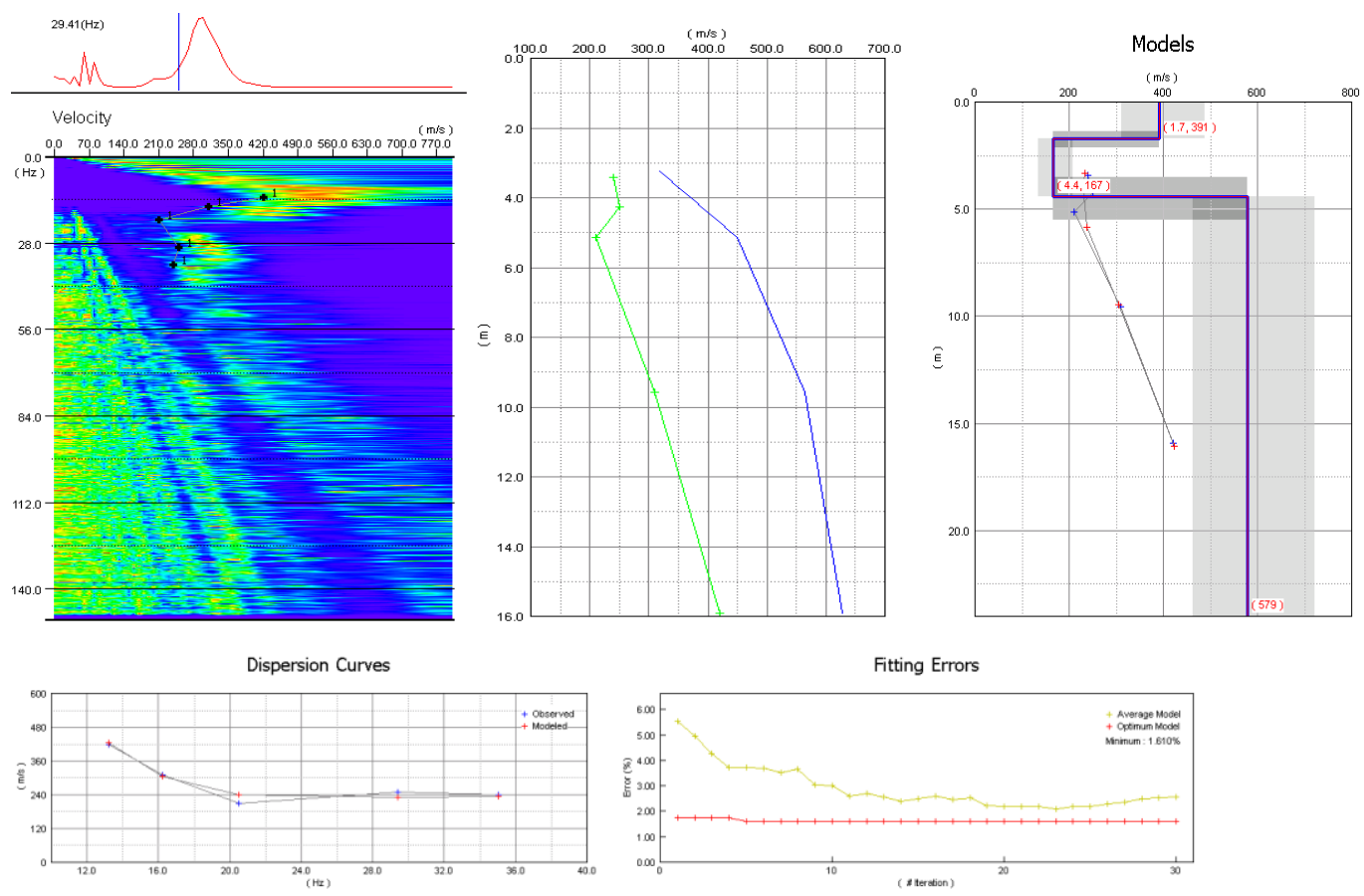


\section{Meter Survey}
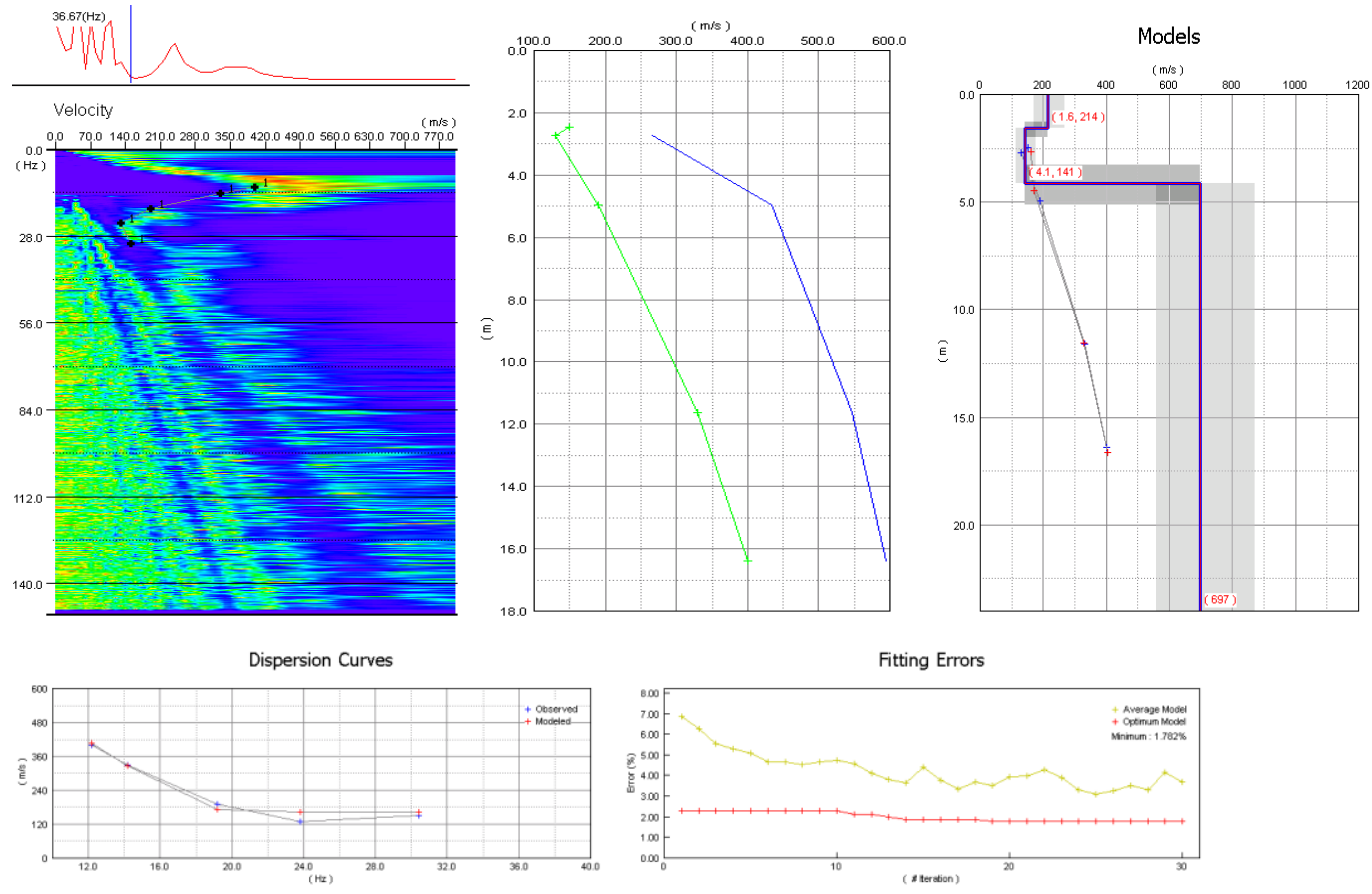

\section{Meter Survey}
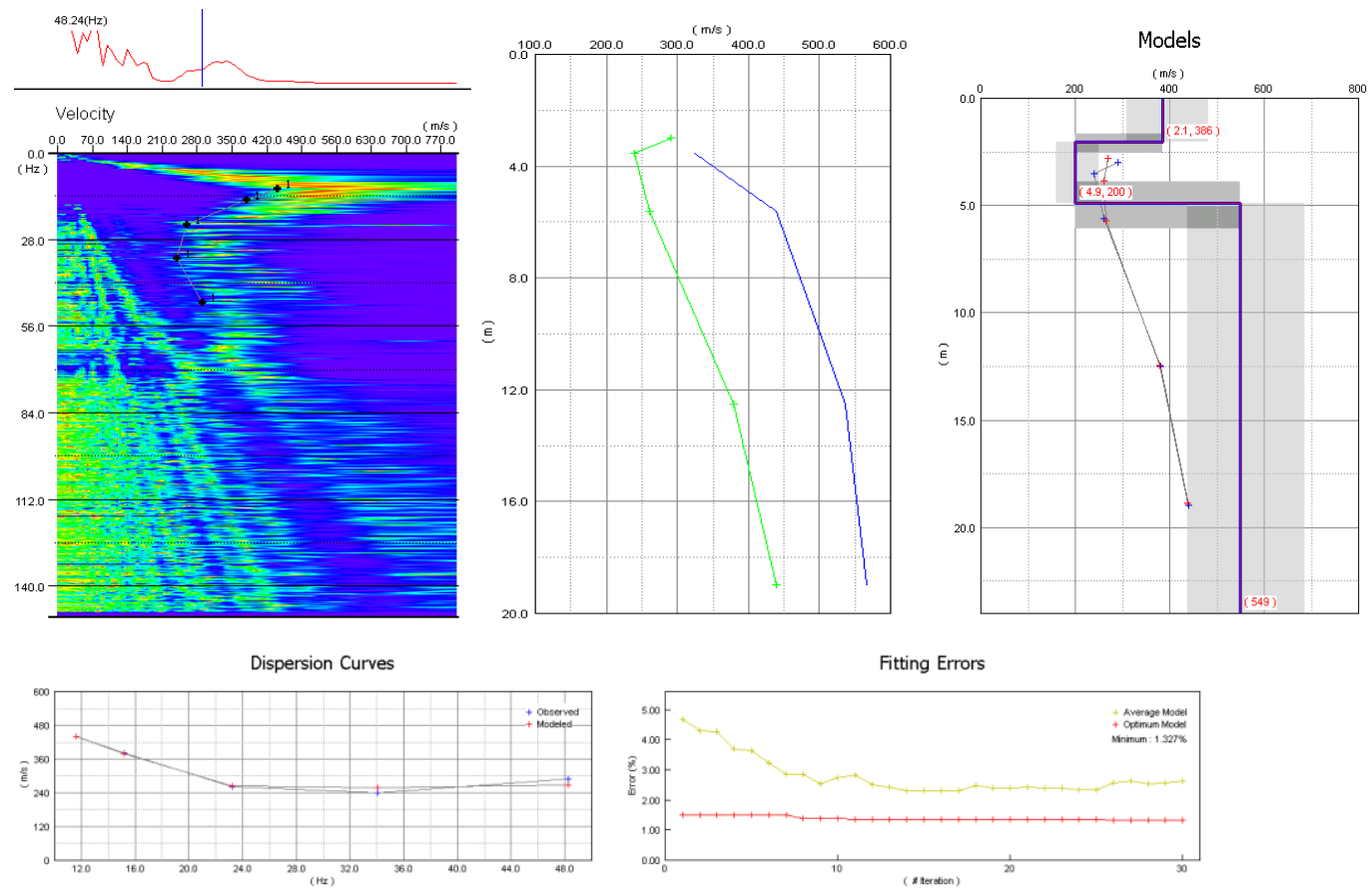
225 Meter Survey
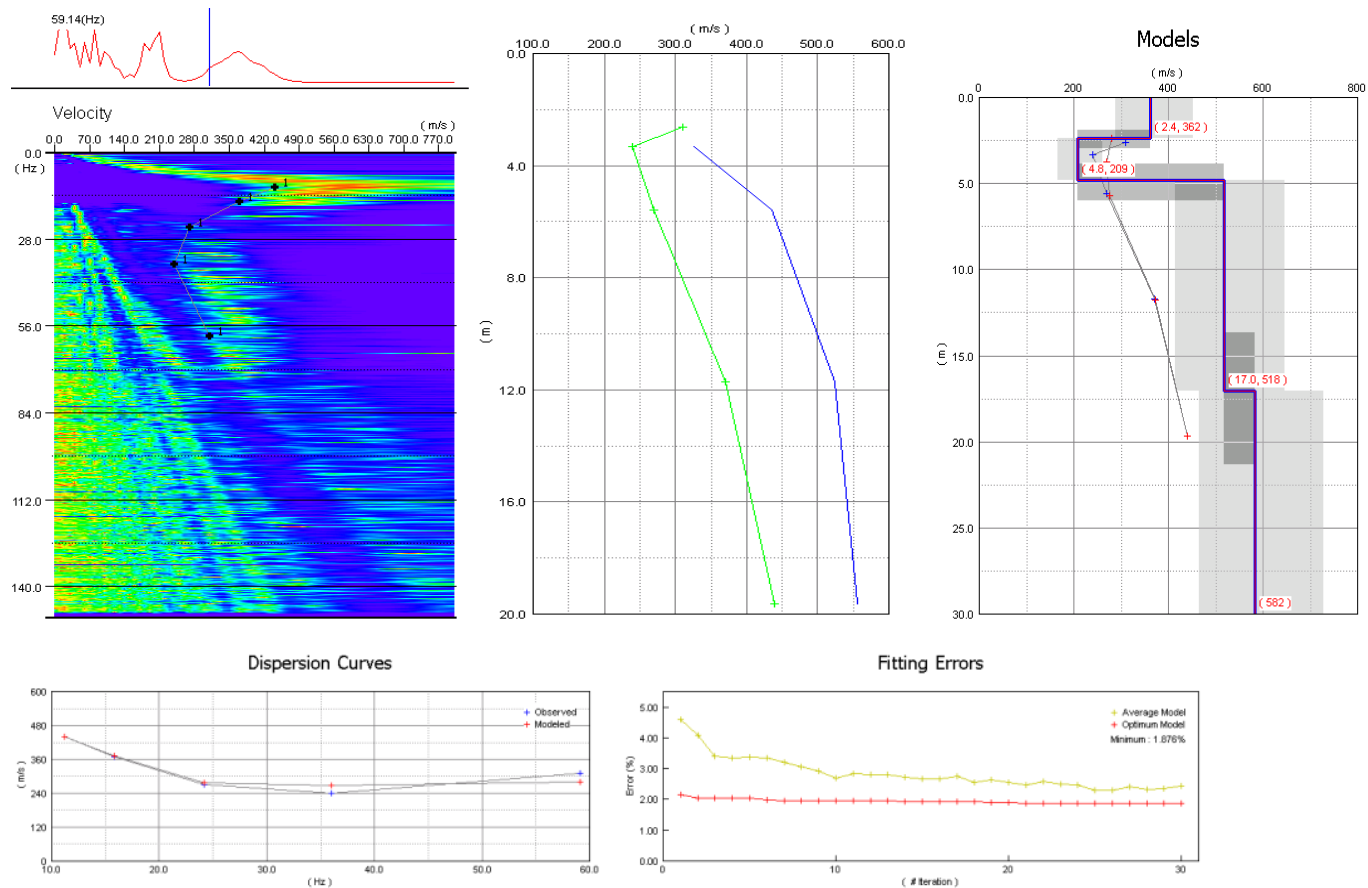

230 Meter Survey
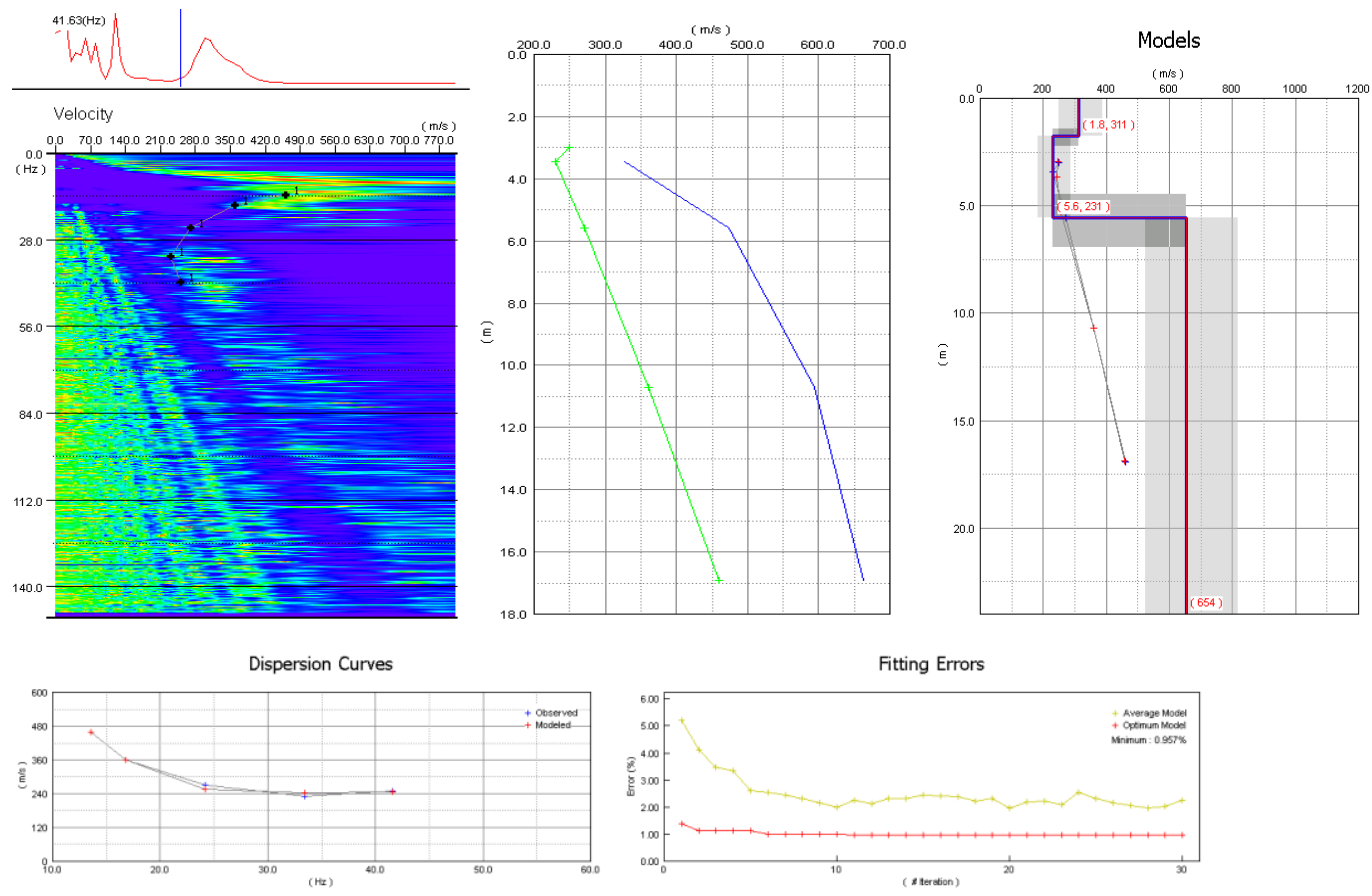
235 Meter Survey
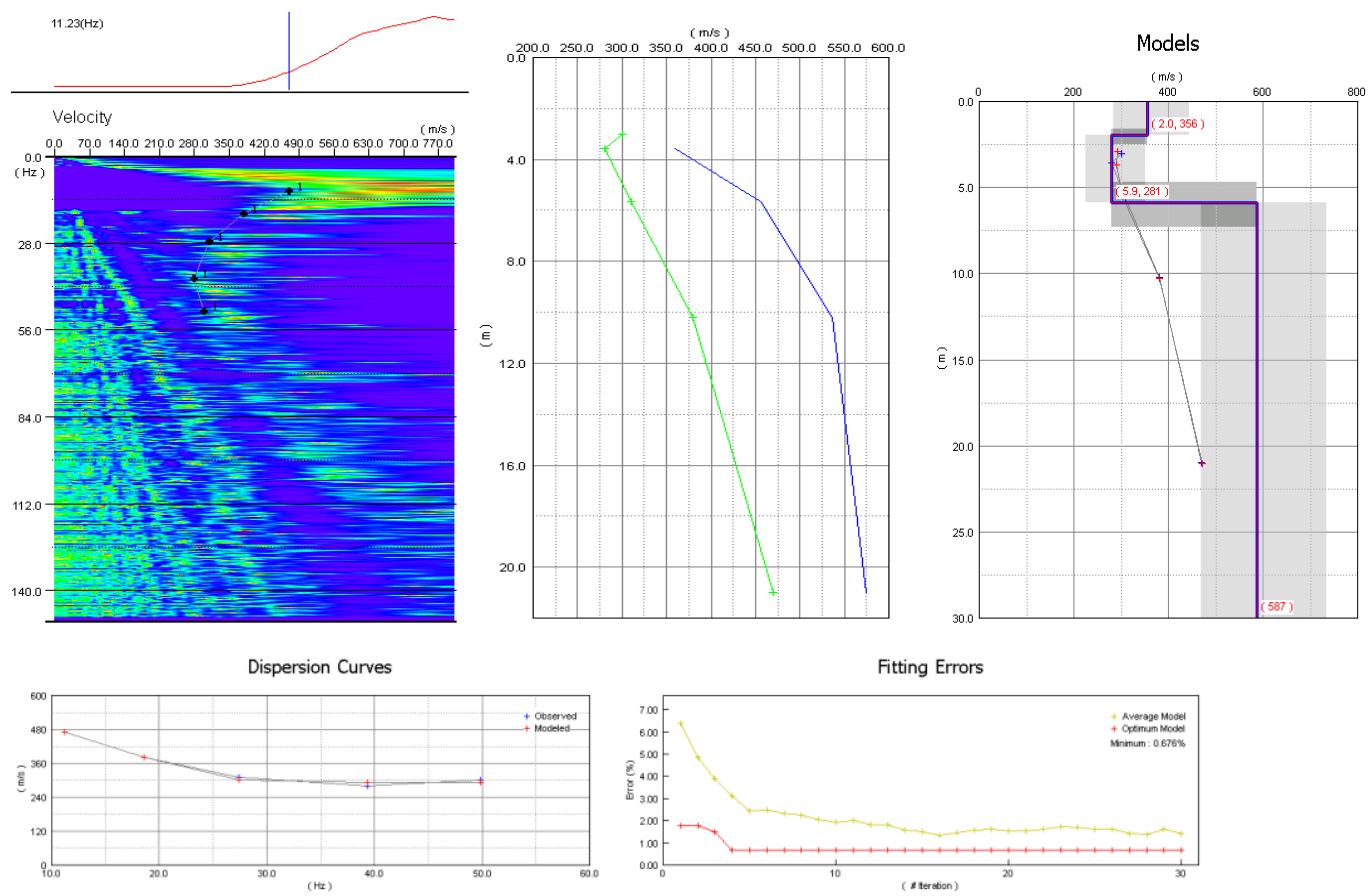

240 Meter Survey
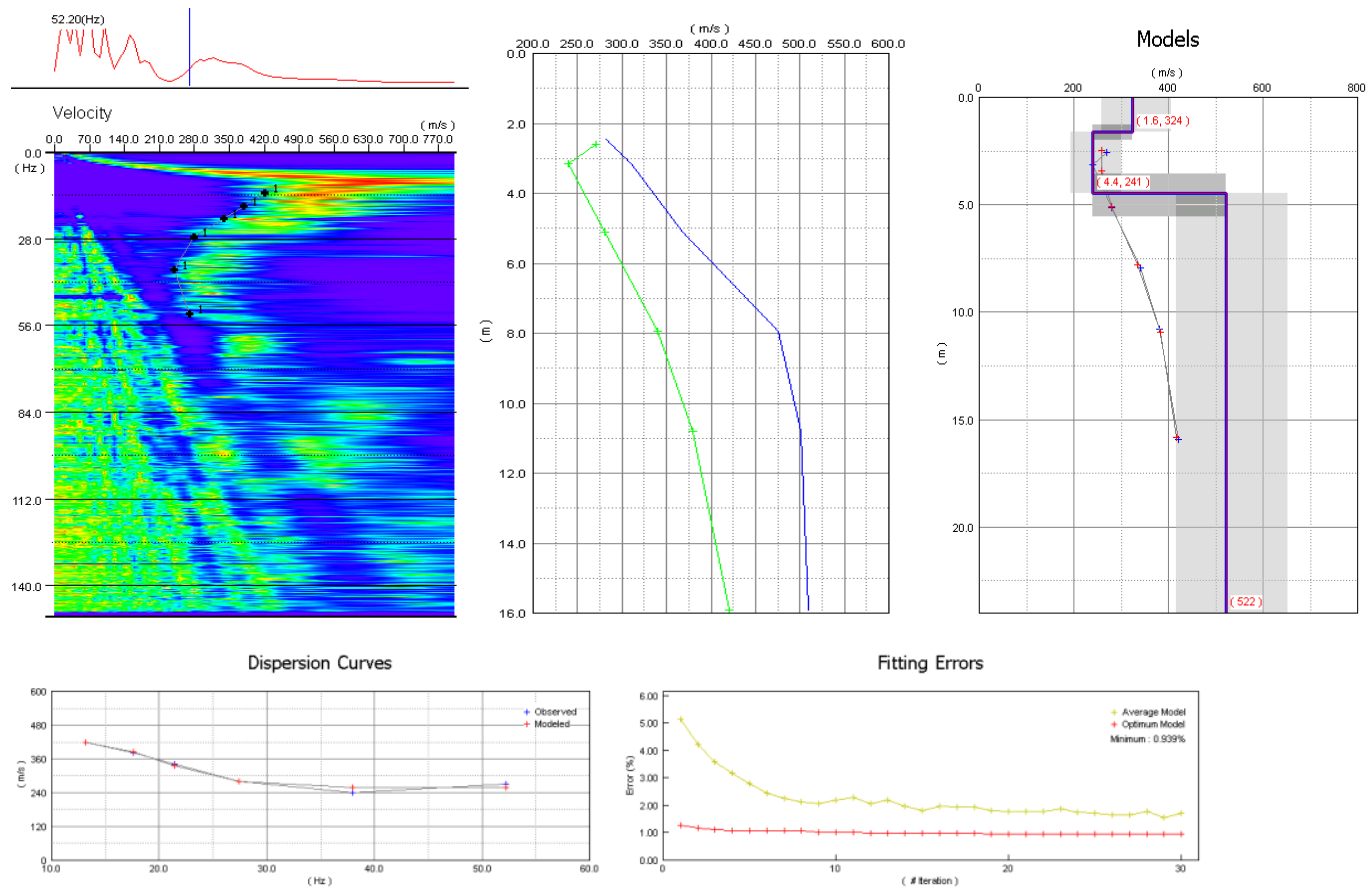
245 Meter Survey
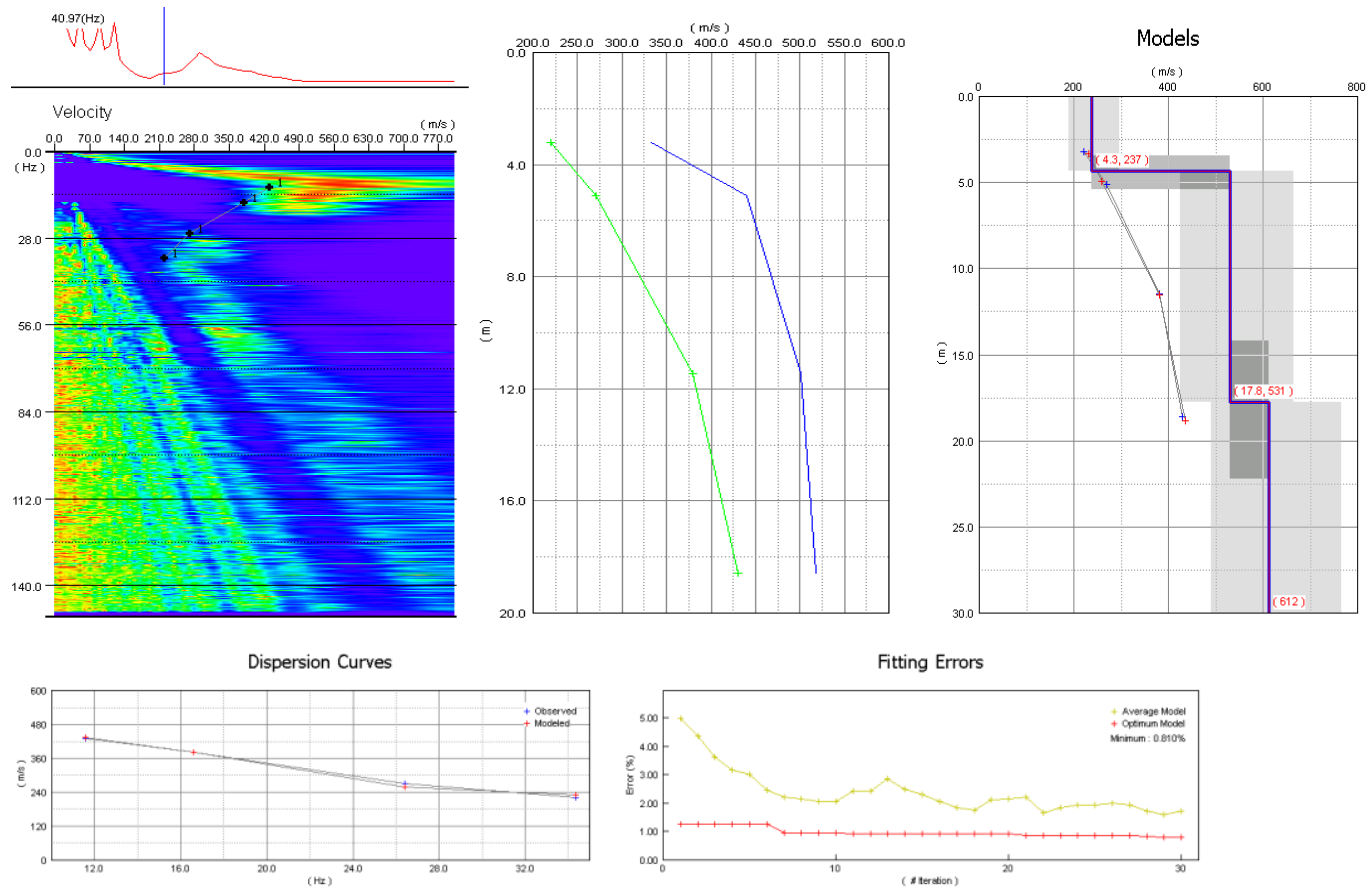

250 Meter Survey
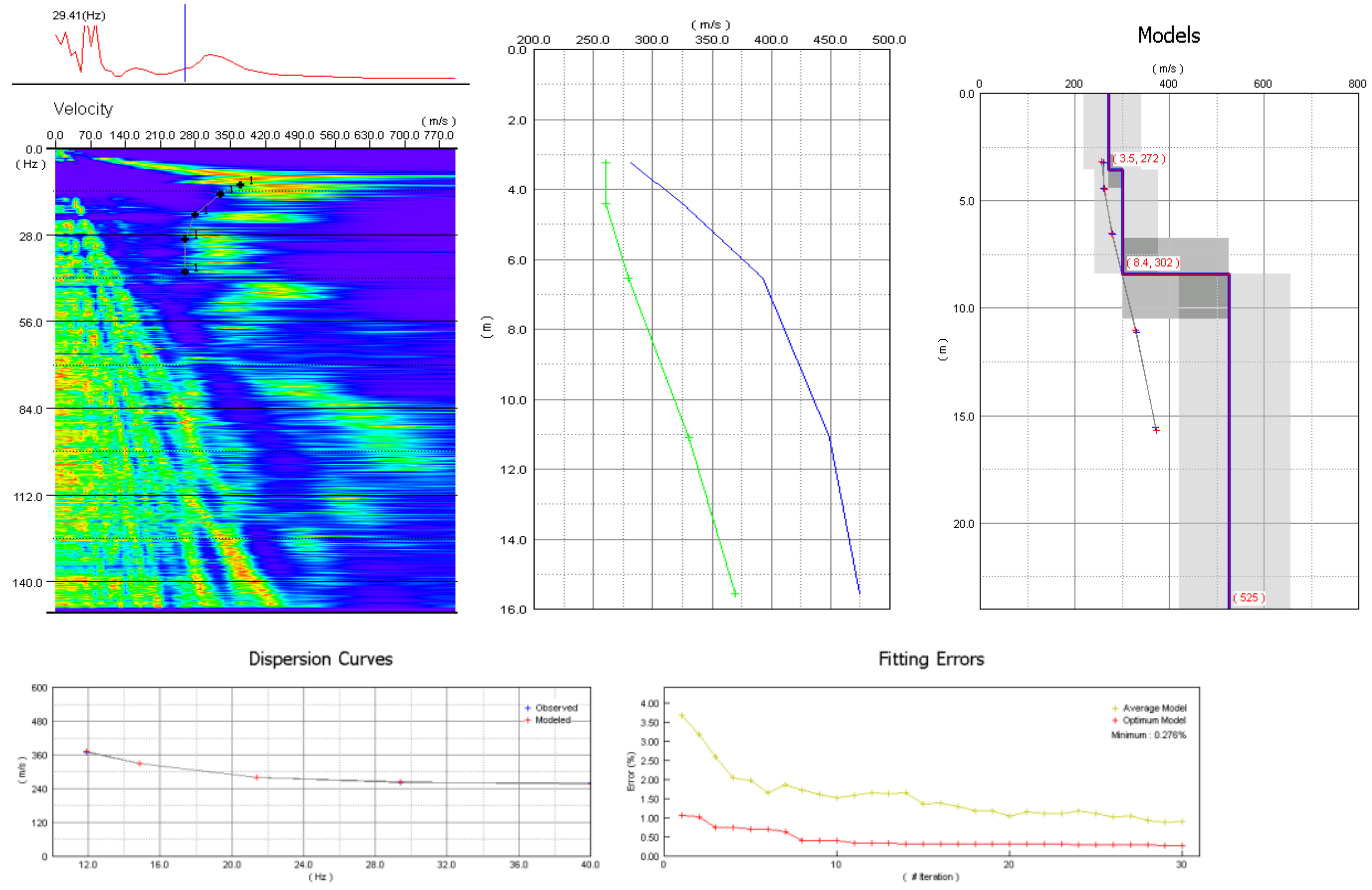


\section{Meter Survey}
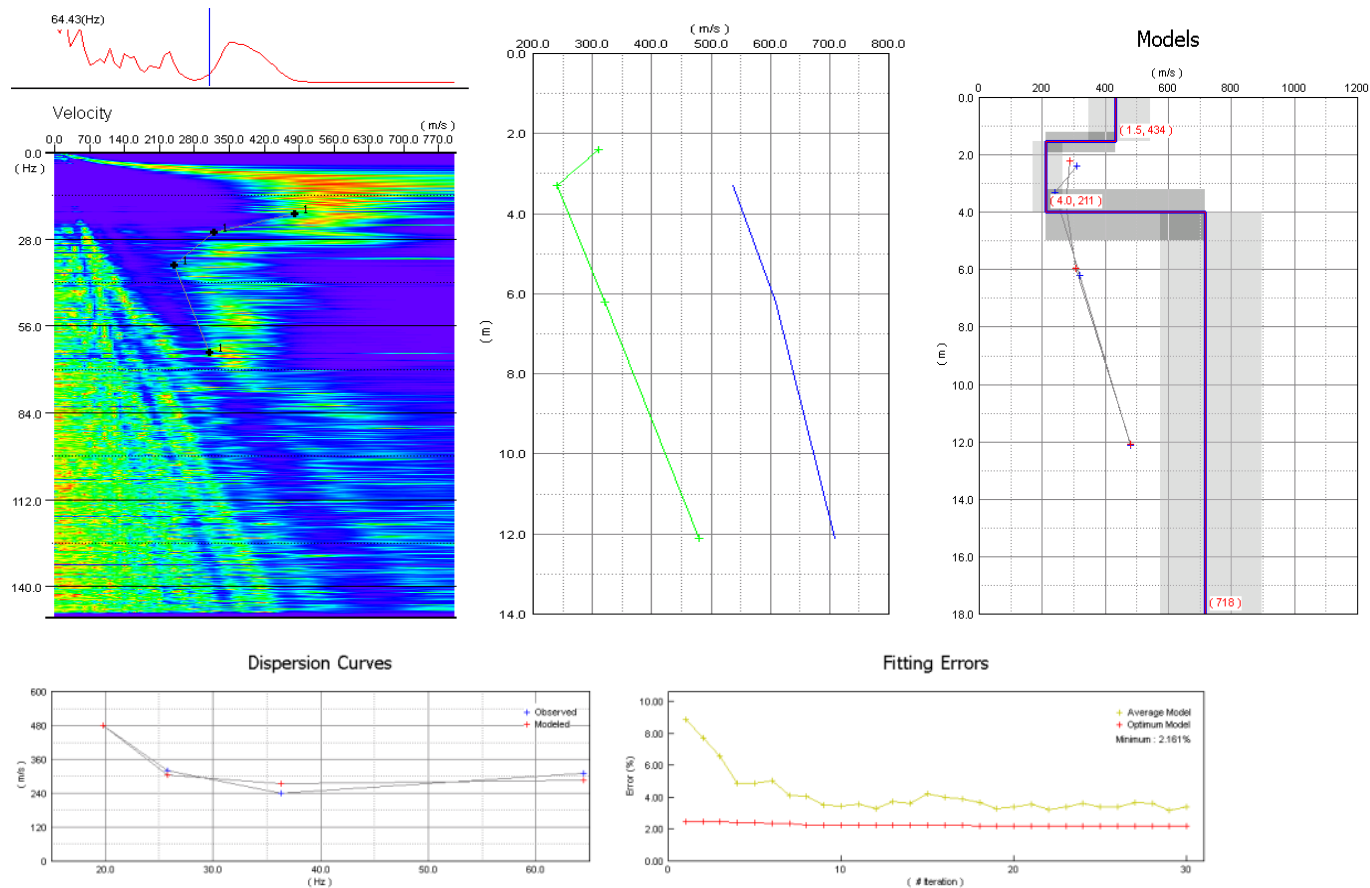

260 Meter Survey

*No $V_{R}$ dispersion curve available.
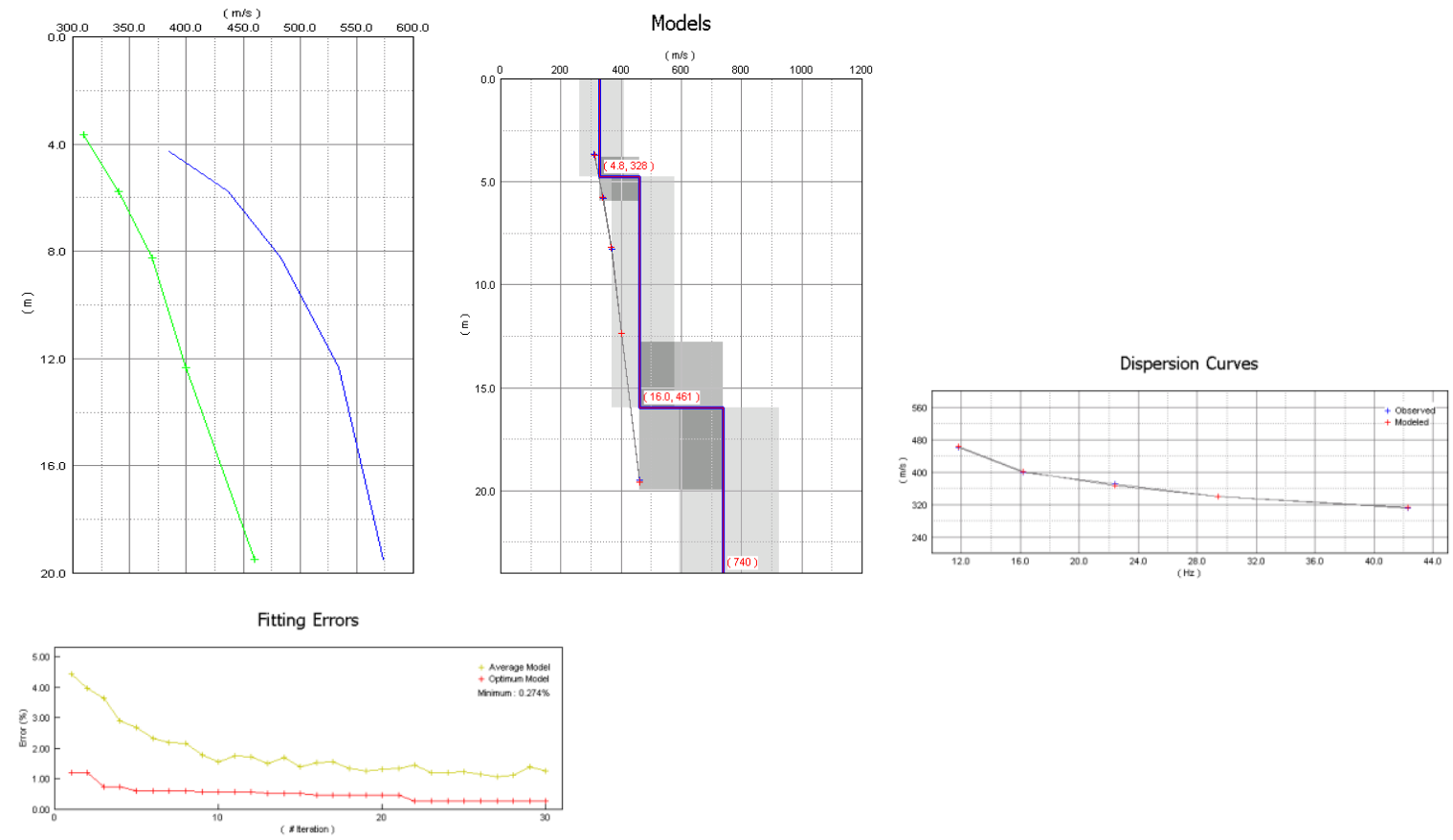
265 Meter Survey
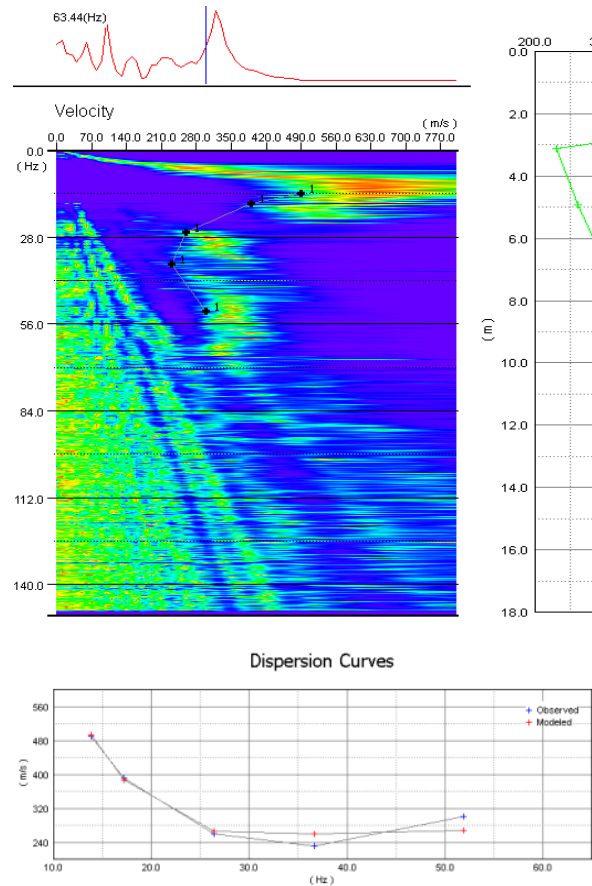
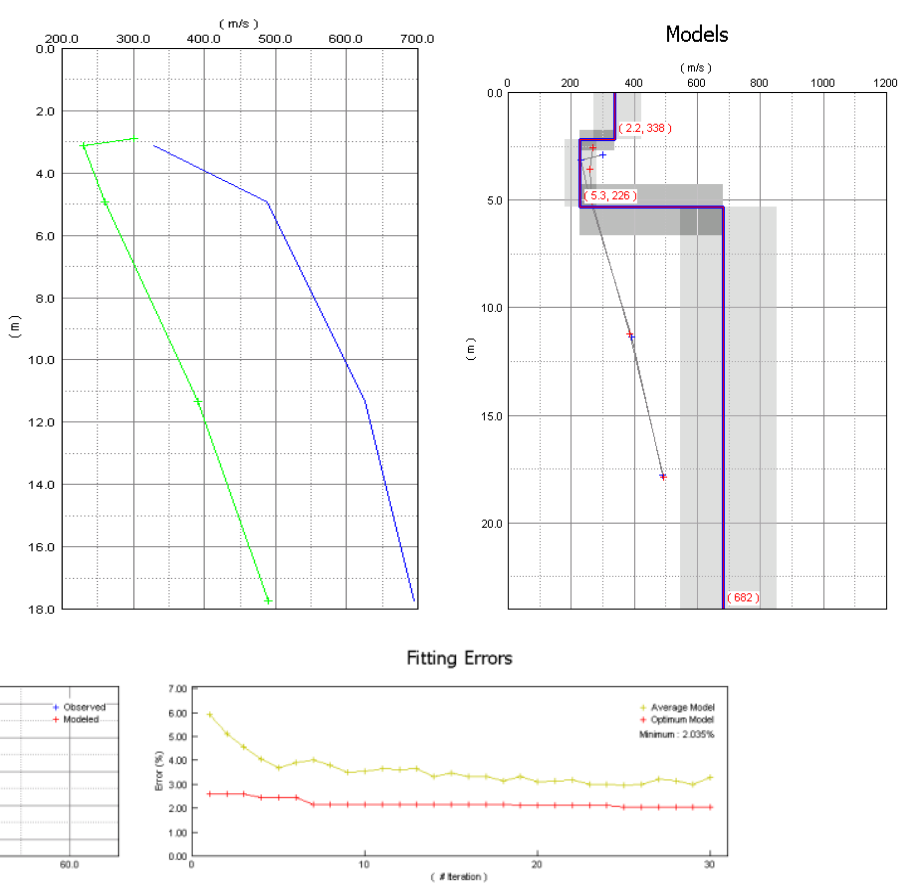

270 Meter Survey

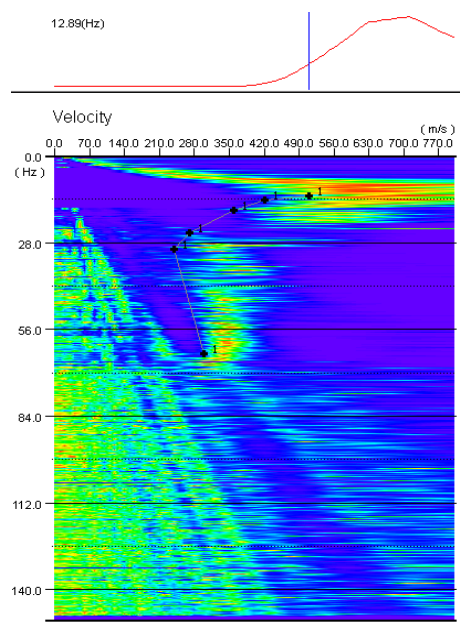

Dispersion Curves

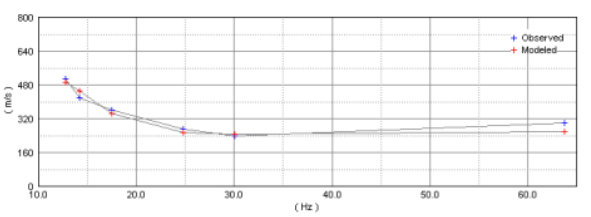

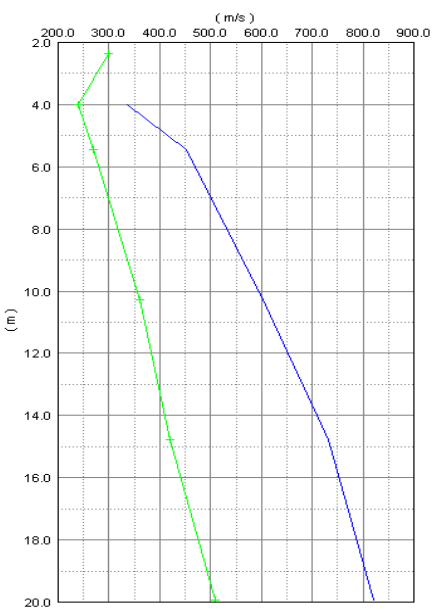

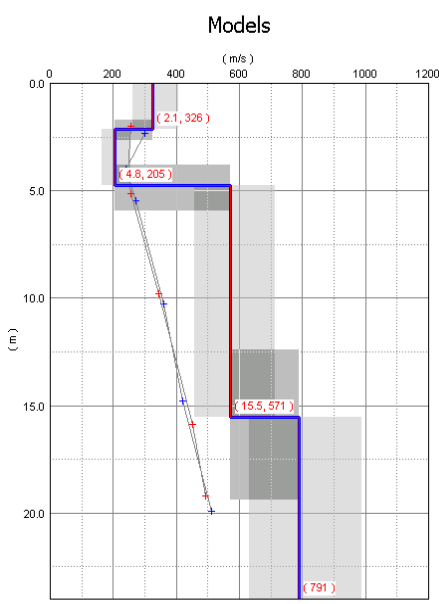

Fitting Errors

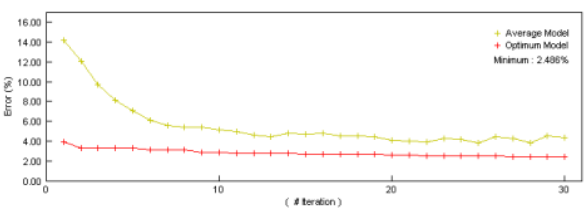


275 Meter Survey
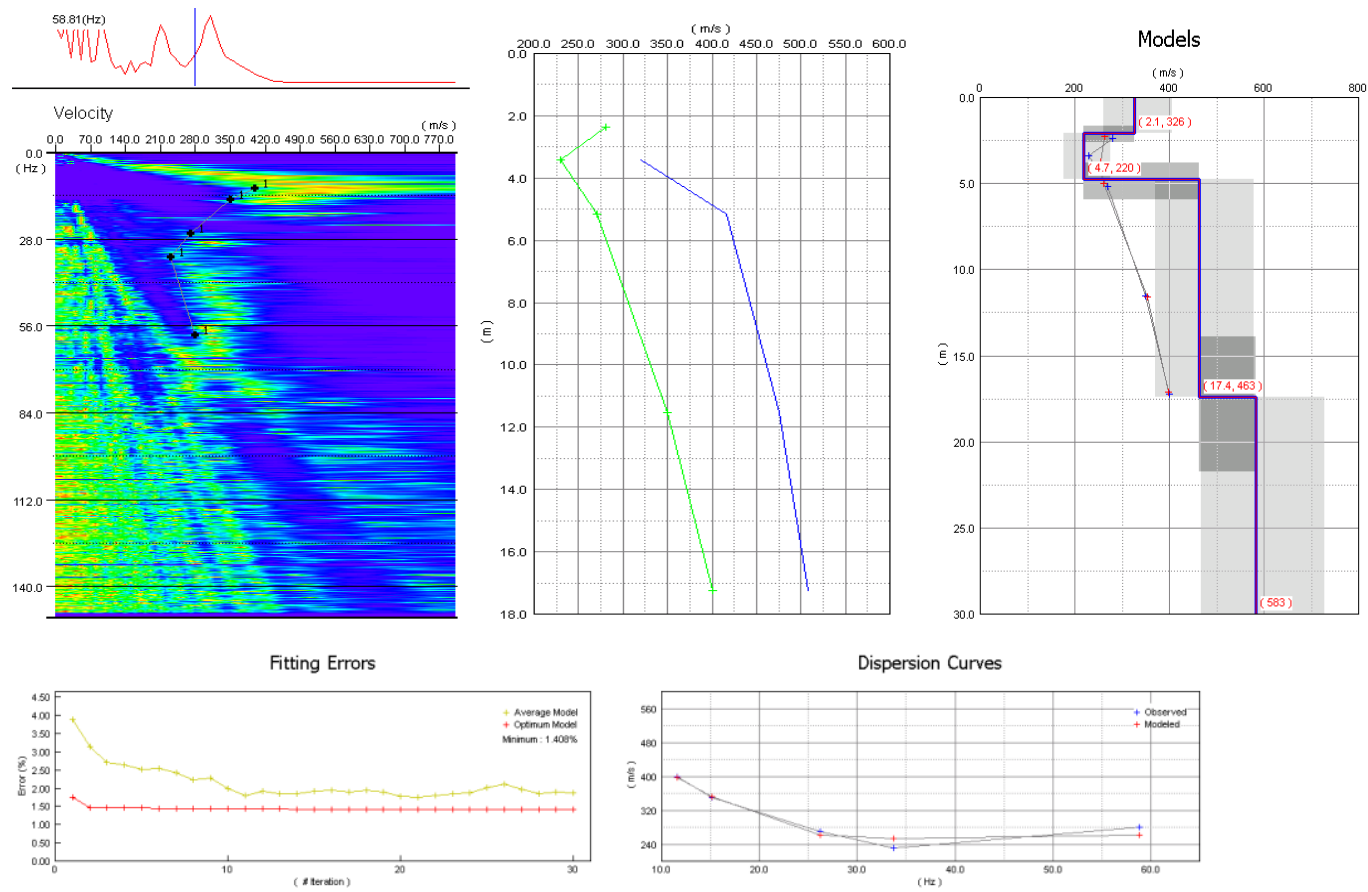

\section{Meter Survey}
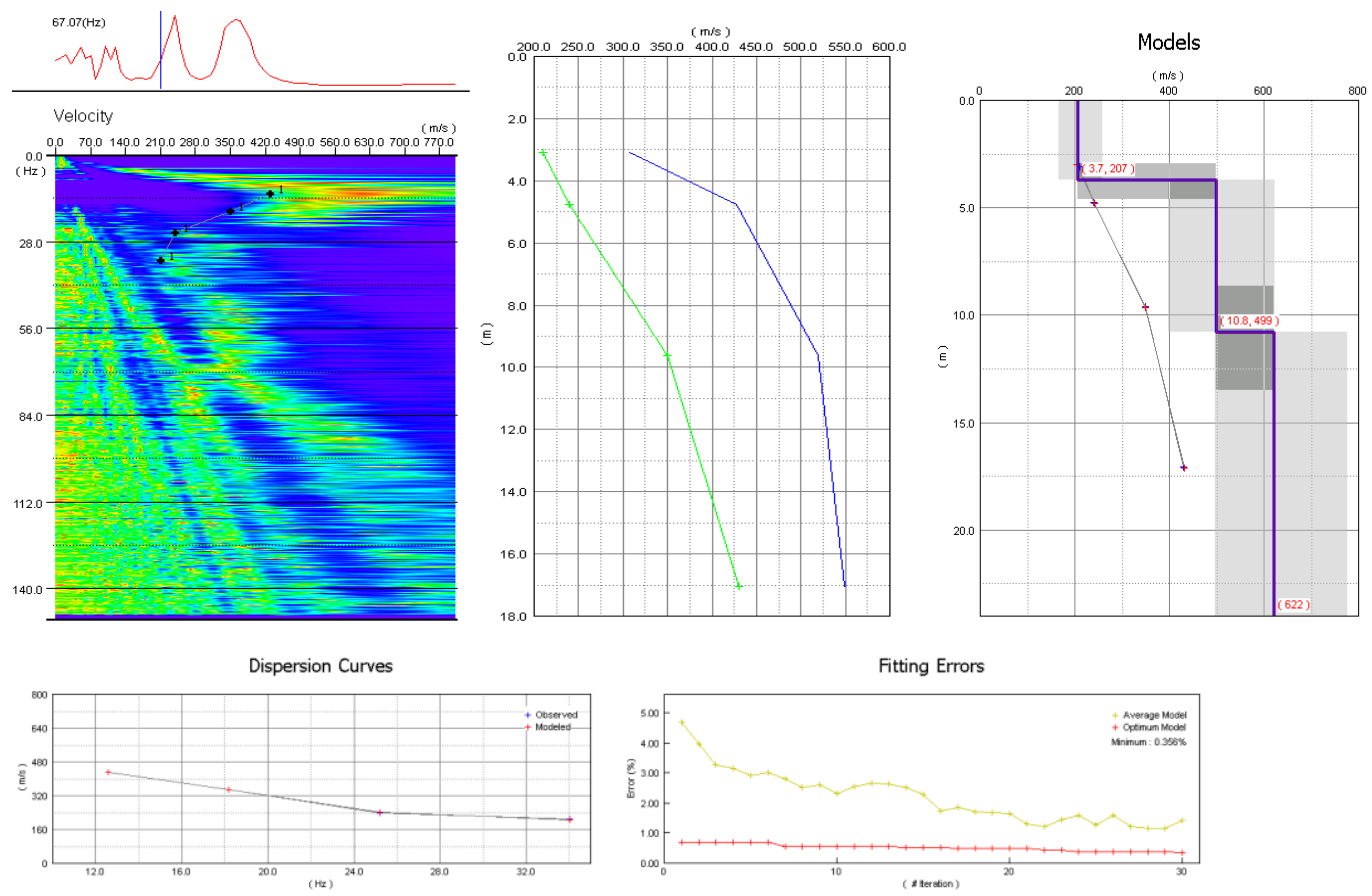
285 Meter Survey
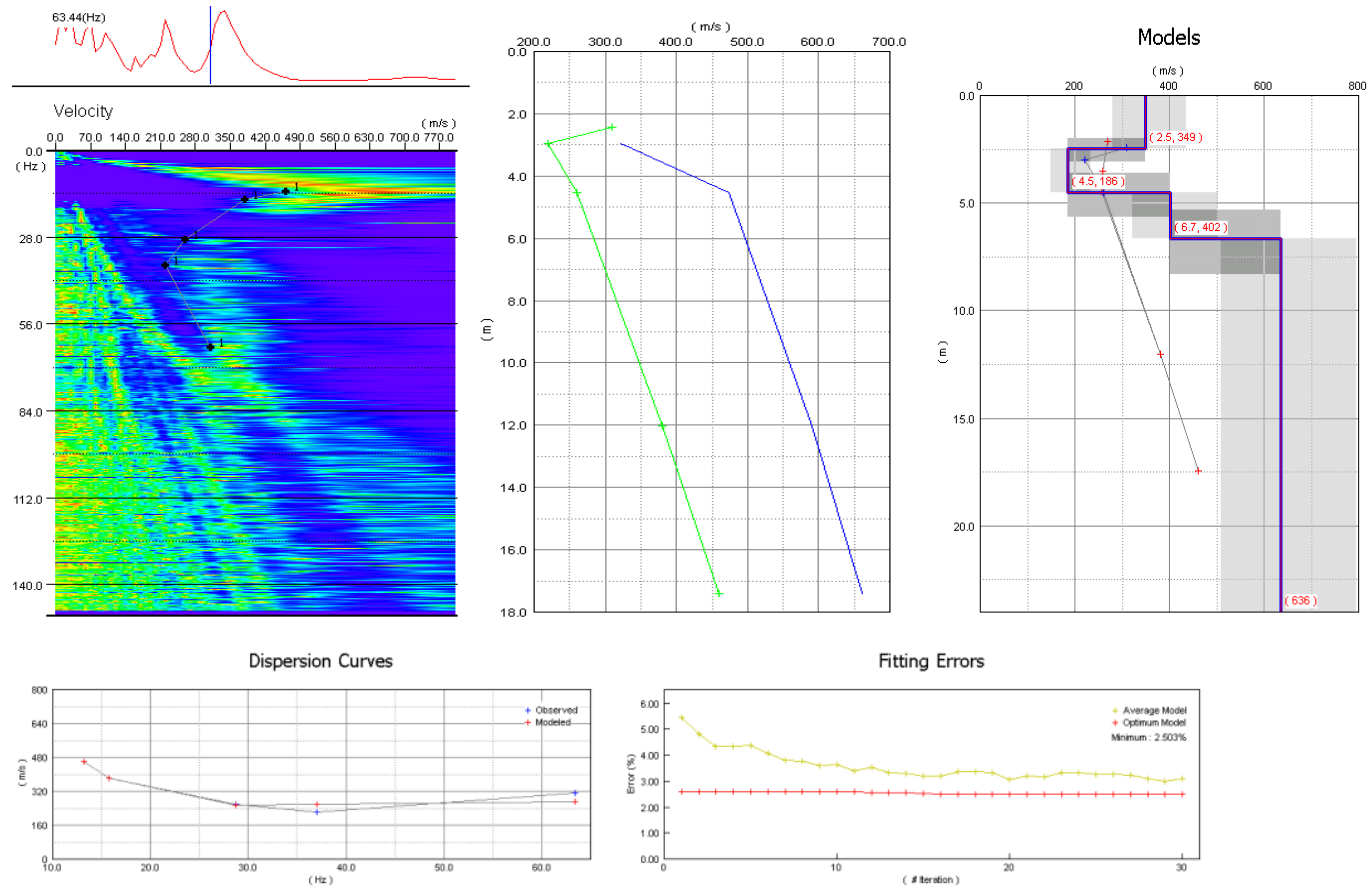

290 Meter Survey
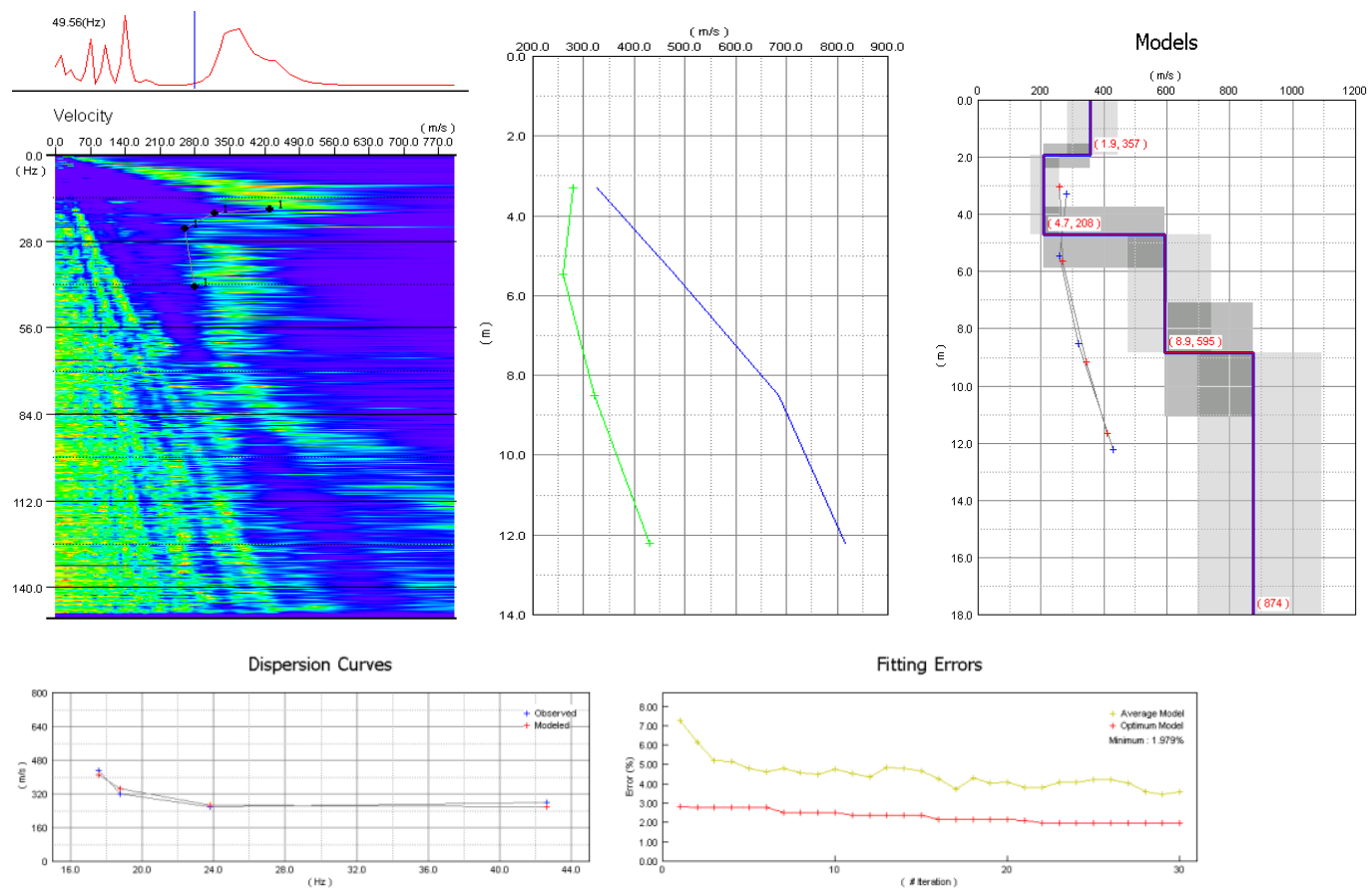


\section{Appendix C: Time weighted average Phase Velocity Values for Each Survey}

The time weighted average phase velocity values for Rayleigh waves and shear waves are shown below. Note that the offset columns refer to the survey locations with respect to the reference stake (see Figure 3.2). The column headers correspond to the depths where the soil columns were analyzed.

\section{Rayleigh wave Values:}

\begin{tabular}{|c|c|c|c|c|c|c|c|}
\hline Offset & $\begin{array}{l}5 \mathrm{~m} \\
(\mathrm{~m} / \mathrm{s})\end{array}$ & $\begin{array}{l}10 \mathrm{~m} \\
(\mathrm{~m} / \mathrm{s})\end{array}$ & $\begin{array}{l}15 \mathrm{~m} \\
(\mathrm{~m} / \mathrm{s})\end{array}$ & Offset & $\begin{array}{l}5 \mathrm{~m} \\
(\mathrm{~m} / \mathrm{s})\end{array}$ & $\begin{array}{l}10 \mathrm{~m} \\
(\mathrm{~m} / \mathrm{s})\end{array}$ & $\begin{array}{l}15 \mathrm{~m} \\
(\mathrm{~m} / \mathrm{s})\end{array}$ \\
\hline-20 & 180 & 210 & 245 & 130 & & 303 & 321 \\
\hline-15 & 174 & 210 & 250 & 135 & 225 & 255 & \\
\hline-10 & 179 & 226 & 268 & 140 & 218 & 251 & \\
\hline-5 & 182 & 224 & 254 & 145 & 195 & 225 & \\
\hline 0 & 213 & 249 & 278 & 150 & 248 & 256 & 277 \\
\hline 5 & 245 & 263 & 289 & 155 & 252 & 253 & 278 \\
\hline 10 & 260 & 280 & 306 & 160 & 230 & 242 & 269 \\
\hline 15 & & 332 & 356 & 165 & 189 & 213 & 244 \\
\hline 20 & 250 & 275 & 306 & 170 & 164 & 197 & 230 \\
\hline 25 & 174 & 222 & 258 & 175 & 200 & 230 & \\
\hline 30 & 295 & 309 & 338 & 180 & 225 & 243 & \\
\hline 35 & 261 & 297 & 329 & 185 & 151 & 182 & 214 \\
\hline 40 & 264 & 293 & 324 & 190 & 243 & 253 & 281 \\
\hline 45 & 258 & 294 & 319 & 195 & 152 & 188 & 221 \\
\hline 50 & 255 & 282 & 312 & 220 & 274 & 294 & 318 \\
\hline 55 & 263 & 280 & 307 & 225 & 277 & 299 & 321 \\
\hline 60 & 176 & 208 & 239 & 230 & 257 & 286 & 315 \\
\hline 65 & 284 & 313 & 337 & 235 & 279 & 310 & 335 \\
\hline 70 & & 305 & 326 & 240 & 271 & 302 & 328 \\
\hline 75 & 222 & 262 & 297 & 245 & 241 & 275 & 303 \\
\hline 80 & 260 & 284 & 308 & 250 & 265 & 283 & 302 \\
\hline 85 & & 290 & & 255 & 293 & 332 & \\
\hline 90 & 213 & 243 & 268 & 260 & 317 & 341 & 360 \\
\hline 95 & 163 & 202 & 236 & 265 & 279 & 305 & 335 \\
\hline 100 & 164 & 209 & 245 & 270 & 278 & 302 & 329 \\
\hline 105 & 247 & 265 & 292 & 275 & 267 & 289 & 310 \\
\hline 110 & 243 & 258 & 286 & 280 & 229 & 265 & 295 \\
\hline 115 & 210 & 231 & 257 & 285 & 273 & 298 & 325 \\
\hline 120 & 180 & 210 & 242 & 290 & 273 & 299 & \\
\hline 125 & 169 & 210 & 246 & & & & \\
\hline \multirow[t]{3}{*}{ Key: } & \multicolumn{2}{|c|}{ Outlier= } & Value & & & & \\
\hline & \multicolumn{2}{|c|}{ Extrapolated= } & Value & & & & \\
\hline & \multicolumn{2}{|c|}{ Not Available= } & & & & & \\
\hline
\end{tabular}


Shear wave Velocity:

\begin{tabular}{|c|c|c|c|c|c|c|c|}
\hline Offset & $\begin{array}{l}5 \mathrm{~m} \\
(\mathrm{~m} / \mathrm{s})\end{array}$ & $\begin{array}{l}10 \mathrm{~m} \\
(\mathrm{~m} / \mathrm{s})\end{array}$ & $\begin{array}{l}15 \mathrm{~m} \\
(\mathrm{~m} / \mathrm{s})\end{array}$ & Offset & $\begin{array}{l}5 \mathrm{~m} \\
(\mathrm{~m} / \mathrm{s})\end{array}$ & $\begin{array}{l}10 \mathrm{~m} \\
(\mathrm{~m} / \mathrm{s})\end{array}$ & $\begin{array}{l}15 \mathrm{~m} \\
(\mathrm{~m} / \mathrm{s})\end{array}$ \\
\hline-20 & 193 & 302 & 384 & 130 & & 319 & 388 \\
\hline-15 & 211 & 334 & 422 & 135 & 245 & 328 & \\
\hline-10 & 236 & 337 & 429 & 140 & 249 & 346 & \\
\hline-5 & 234 & 350 & 360 & 145 & 239 & 292 & \\
\hline 0 & 255 & 312 & 367 & 150 & 240 & 293 & 345 \\
\hline 5 & 259 & 310 & 383 & 155 & 232 & 296 & 373 \\
\hline 10 & 259 & 334 & 377 & 160 & 220 & 307 & 369 \\
\hline 15 & & 376 & 424 & 165 & 192 & 288 & 345 \\
\hline 20 & 248 & 345 & 395 & 170 & 193 & 297 & 362 \\
\hline 25 & 240 & 329 & 378 & 175 & 206 & 325 & \\
\hline 30 & 293 & 368 & 418 & 180 & 234 & 289 & \\
\hline 35 & 295 & 385 & 429 & 185 & 178 & 274 & 334 \\
\hline 40 & 298 & 346 & 415 & 190 & 232 & 331 & 386 \\
\hline 45 & 293 & 375 & 415 & 195 & 189 & 297 & 367 \\
\hline 50 & 266 & 363 & 412 & 220 & 301 & 371 & 410 \\
\hline 55 & 262 & 343 & 399 & 225 & 313 & 375 & 408 \\
\hline 60 & 190 & 283 & 354 & 230 & 287 & 375 & 429 \\
\hline 65 & 324 & 368 & 418 & 235 & 299 & 401 & 442 \\
\hline 70 & & 353 & 407 & 240 & 325 & 386 & 418 \\
\hline 75 & 254 & 350 & 417 & 245 & 301 & 368 & 404 \\
\hline 80 & 268 & 355 & 391 & 250 & 286 & 334 & 374 \\
\hline 85 & & 328 & & 255 & 359 & 454 & \\
\hline 90 & 238 & 298 & 346 & 260 & 361 & 397 & 422 \\
\hline 95 & 209 & 289 & 364 & 265 & 308 & 399 & 455 \\
\hline 100 & 214 & 326 & 404 & 270 & 298 & 372 & 422 \\
\hline 105 & 269 & 332 & 404 & 275 & 301 & 353 & 379 \\
\hline 110 & 255 & 326 & 392 & 280 & 286 & 353 & 404 \\
\hline 115 & 230 & 311 & 347 & 285 & 293 & 378 & 429 \\
\hline 120 & 188 & 294 & 376 & 290 & 309 & 408 & \\
\hline 125 & 209 & 281 & 362 & & & & \\
\hline \multirow[t]{3}{*}{ Key: } & \multicolumn{2}{|c|}{ Outlier= } & Value & & & & \\
\hline & \multicolumn{2}{|c|}{ Extrapolated= } & Value & & & & \\
\hline & \multicolumn{2}{|c|}{ Not Available $=$} & & & & & \\
\hline
\end{tabular}




\section{Appendix D: MATALB Output: Semi-variograms, Covariance Functions, and \\ Correlograms}

The following section contains empirical semi-variograms, covariance functions, correlograms, semi-variograms fitted with spherical models, semi-variograms fitted with exponential models, and semi-variograms fitted with Gaussian models for each depth and each differing amount of data. Note that the modeled semi-variograms are shown in the order of the spherical model, then exponential model, and then Gaussian model. 


\section{5m Rayleigh wave Velocity with All Data}
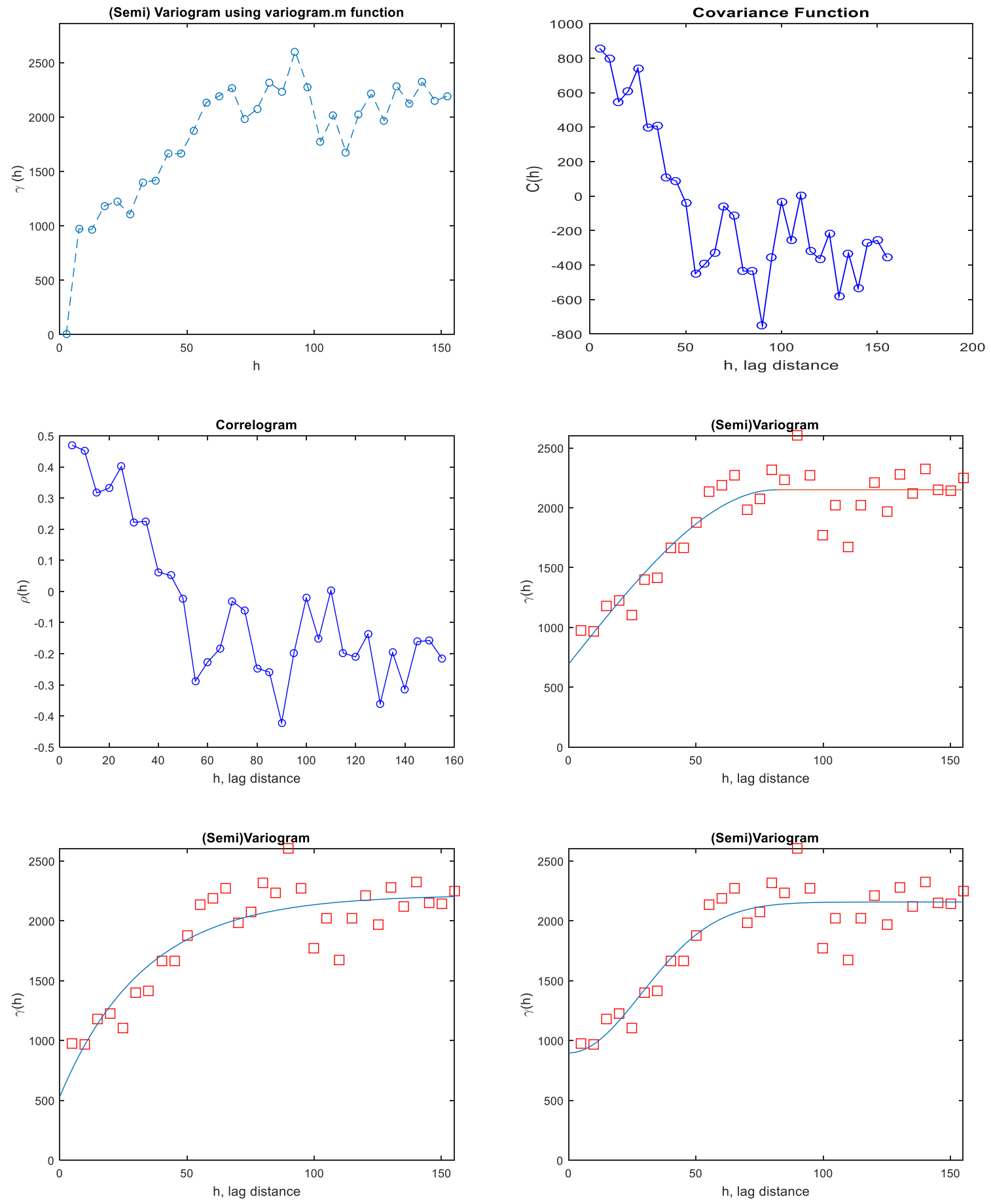


\section{0m Rayleigh wave Velocity with All Data}
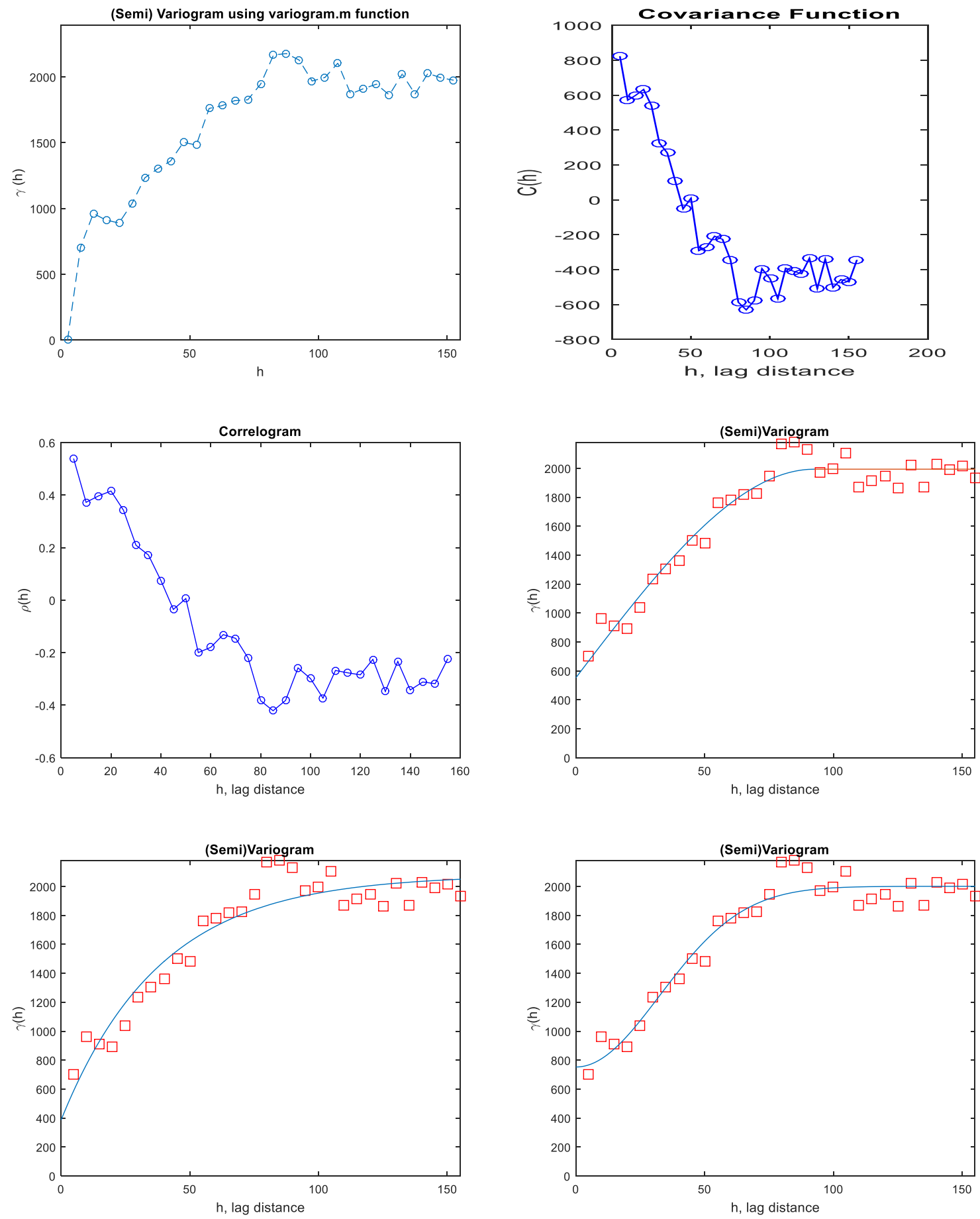


\section{5m Rayleigh wave Velocity with All Data}
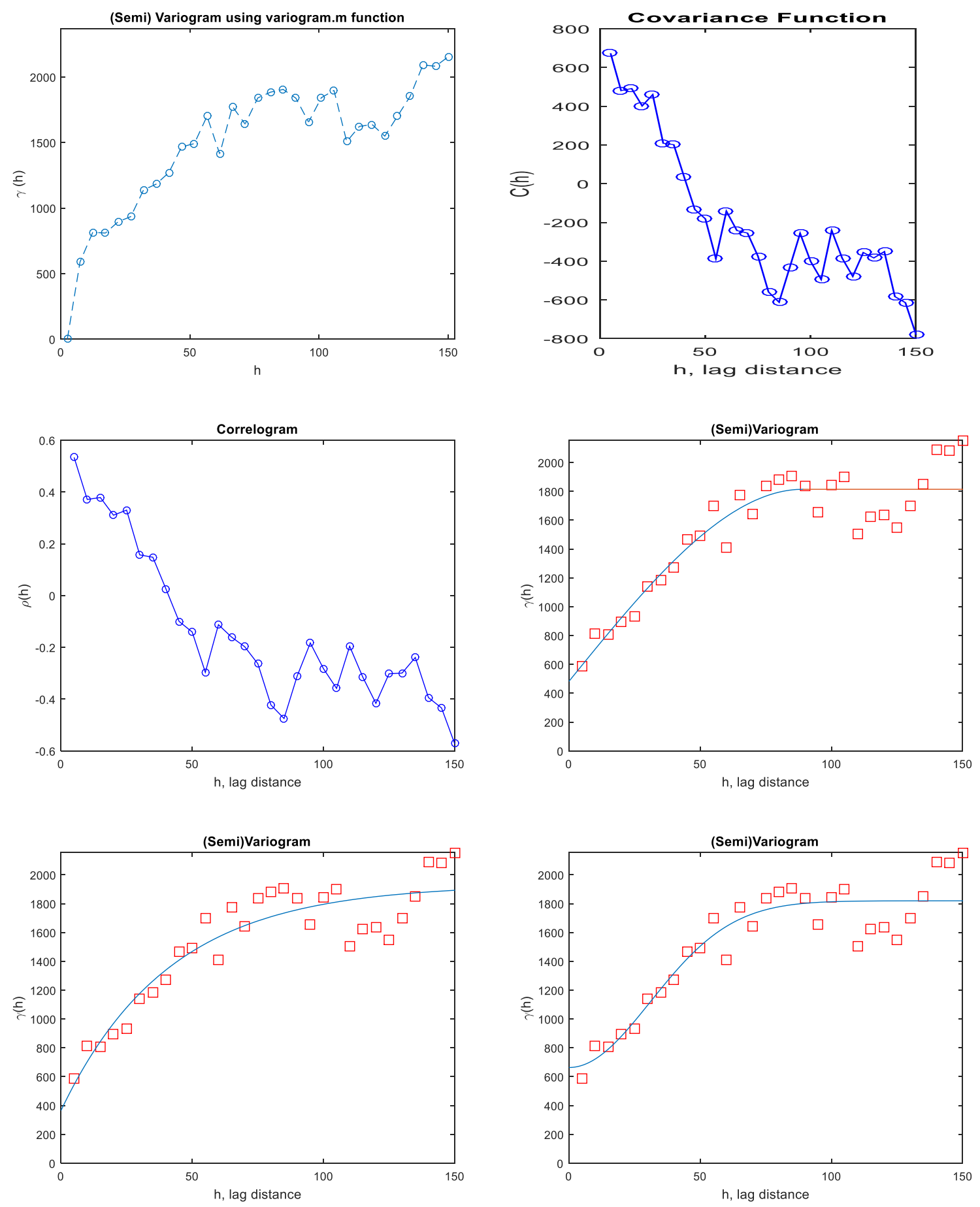


\section{5m Shear wave Velocity with All Data}
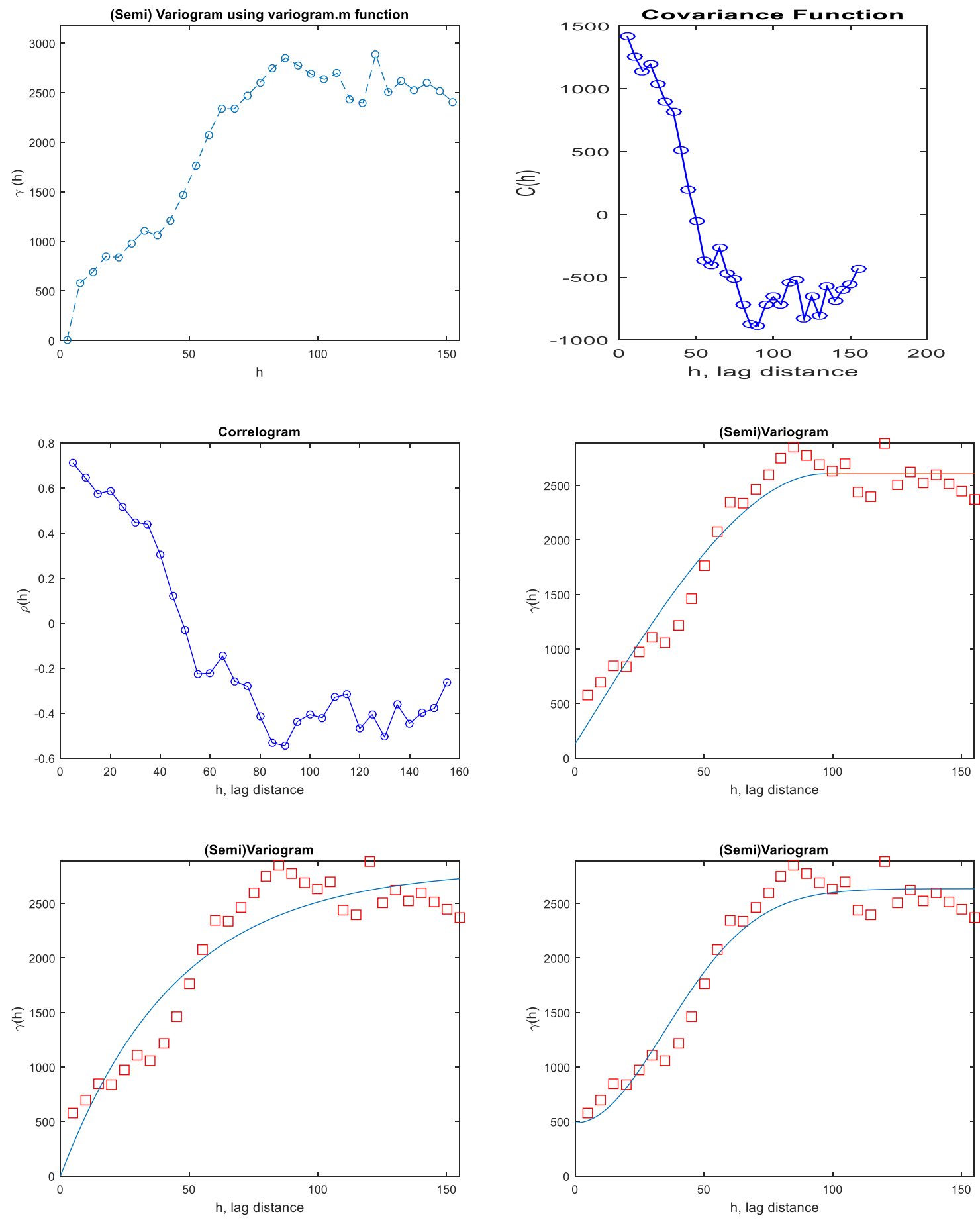


\section{0m Shear wave Velocity with All Data}
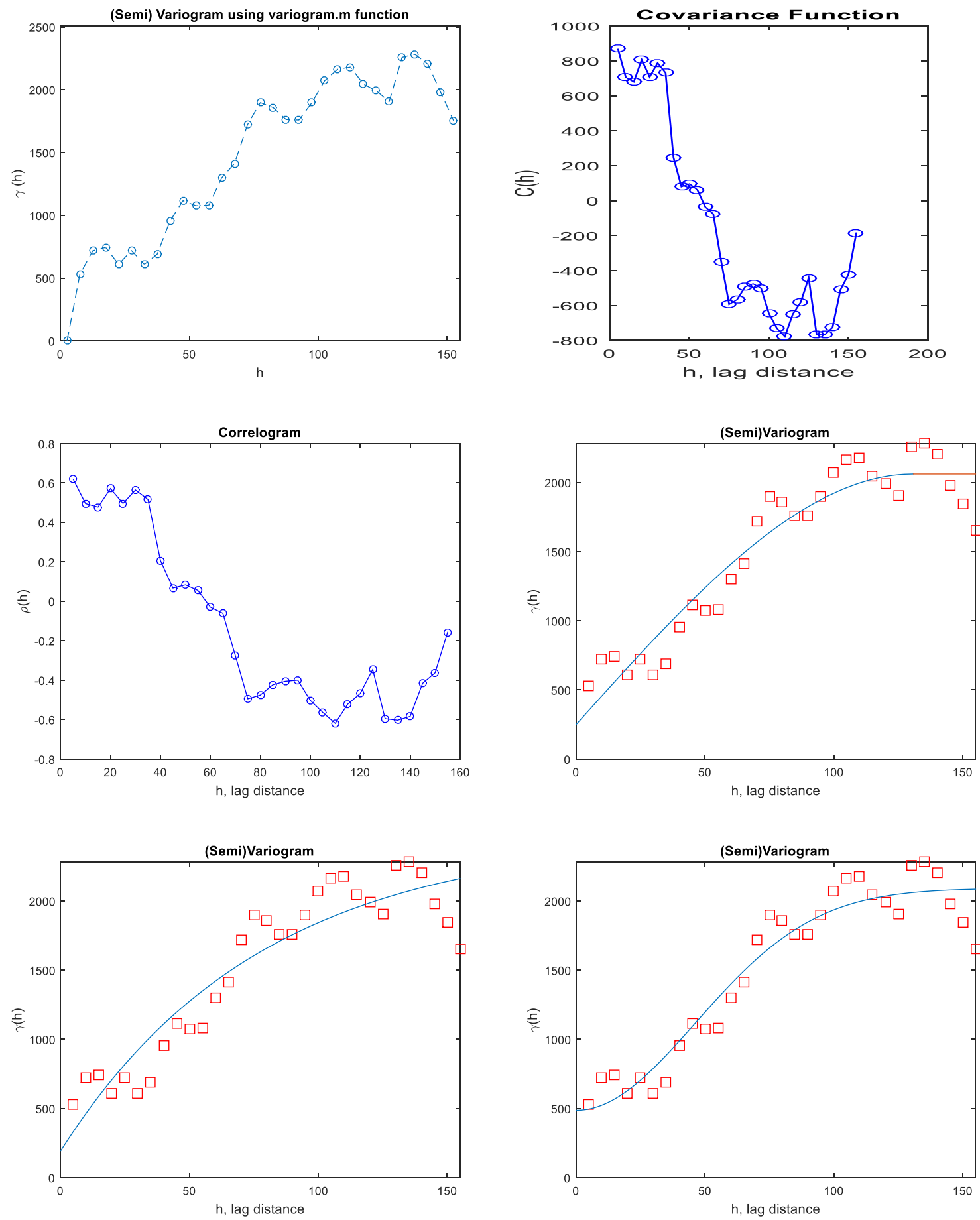


\section{5m Shear wave Velocity with All Data}
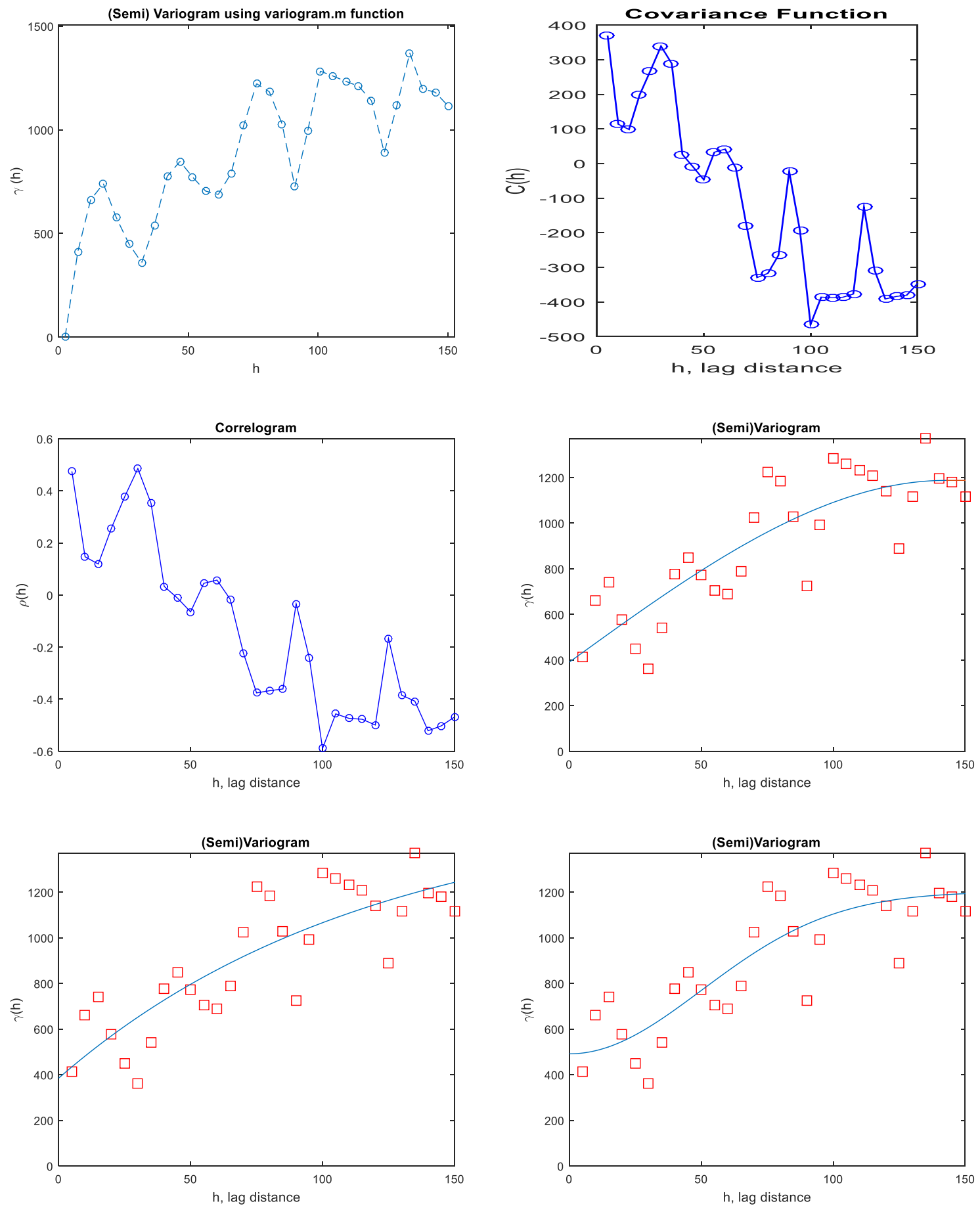


\section{5m Rayleigh wave Velocity without Data after Hill}
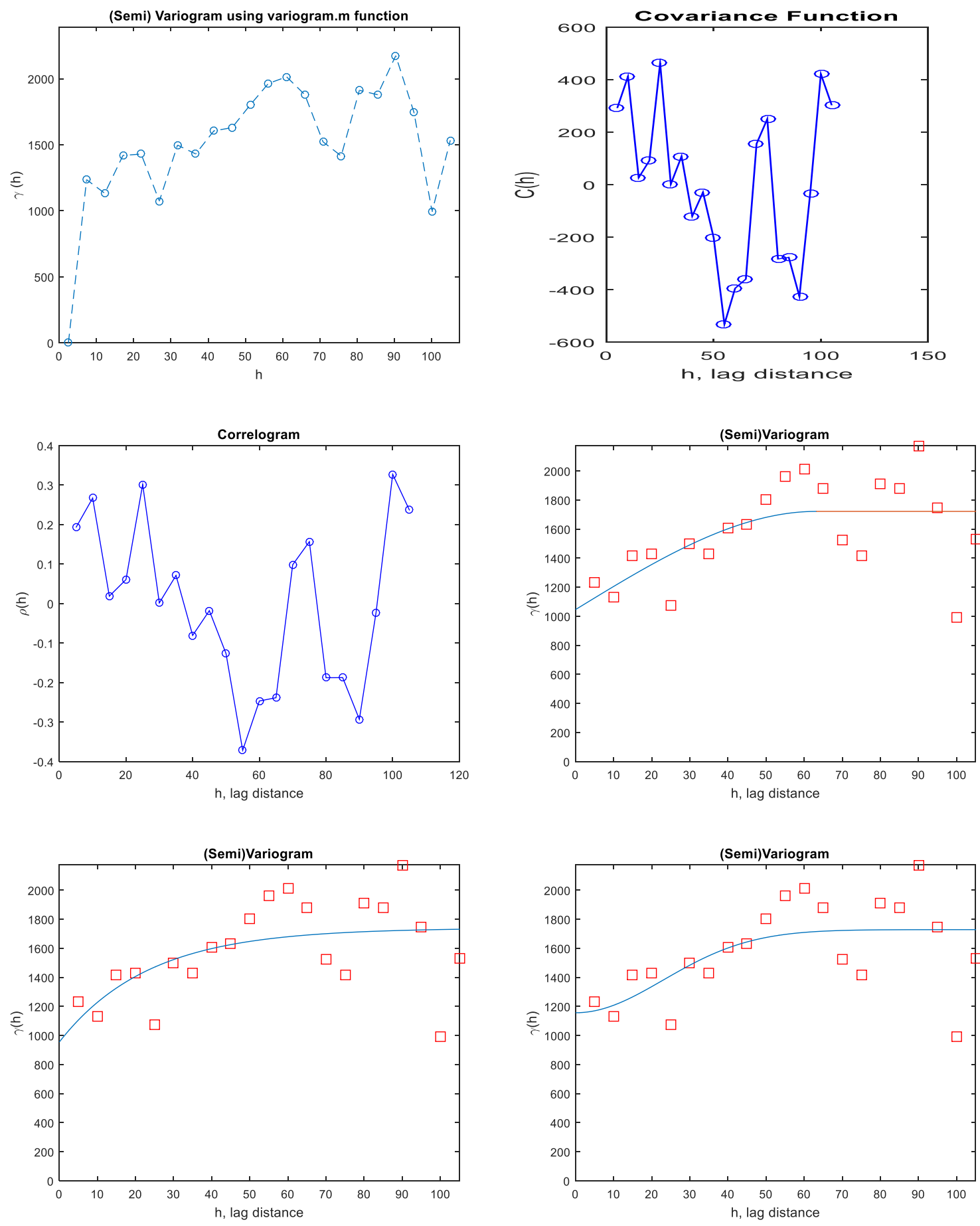


\section{0m Rayleigh wave Velocity without Data after Hill}
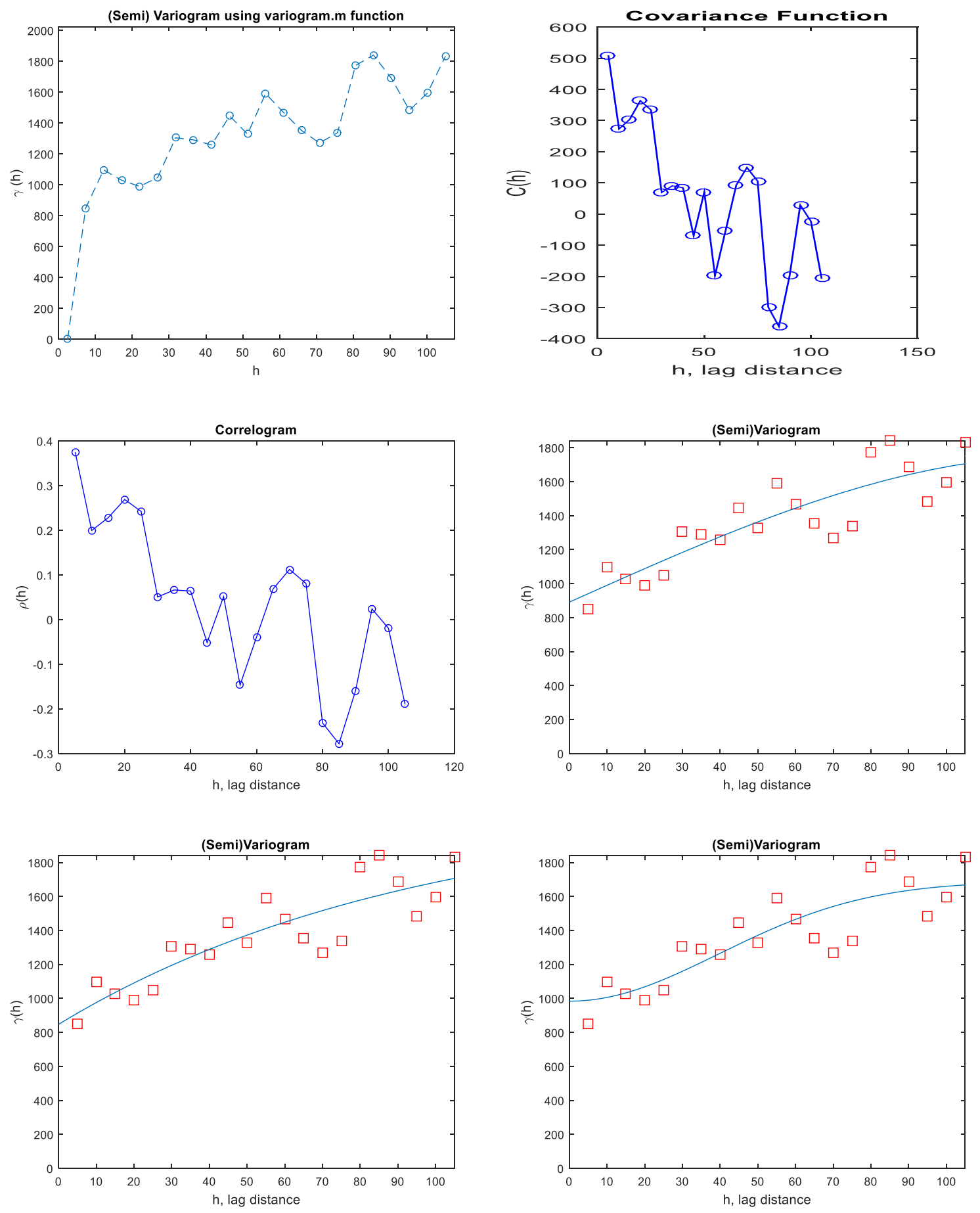


\section{5m Rayleigh wave Velocity without Data after Hill}
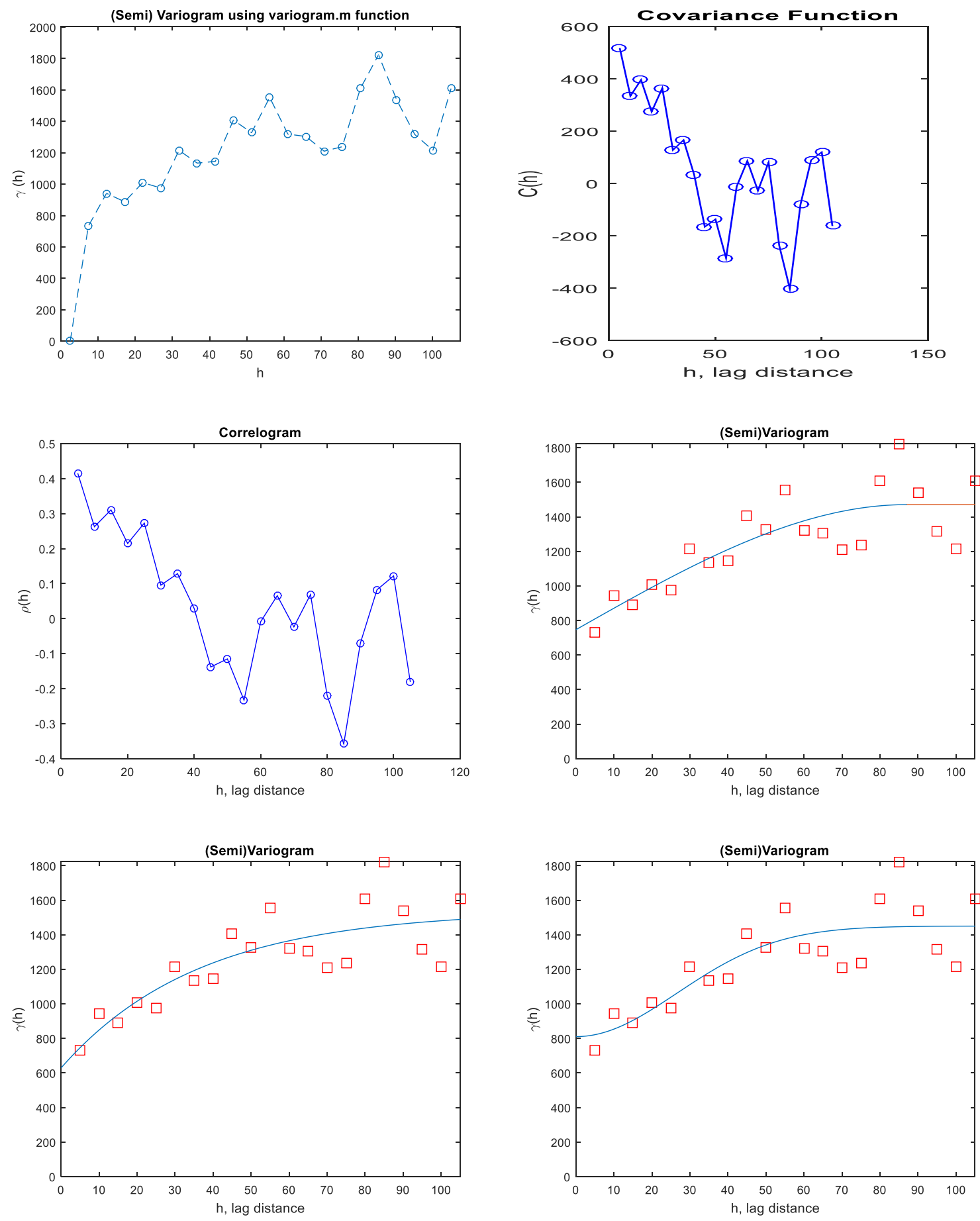


\section{5m Shear wave Velocity without Data after Hill}
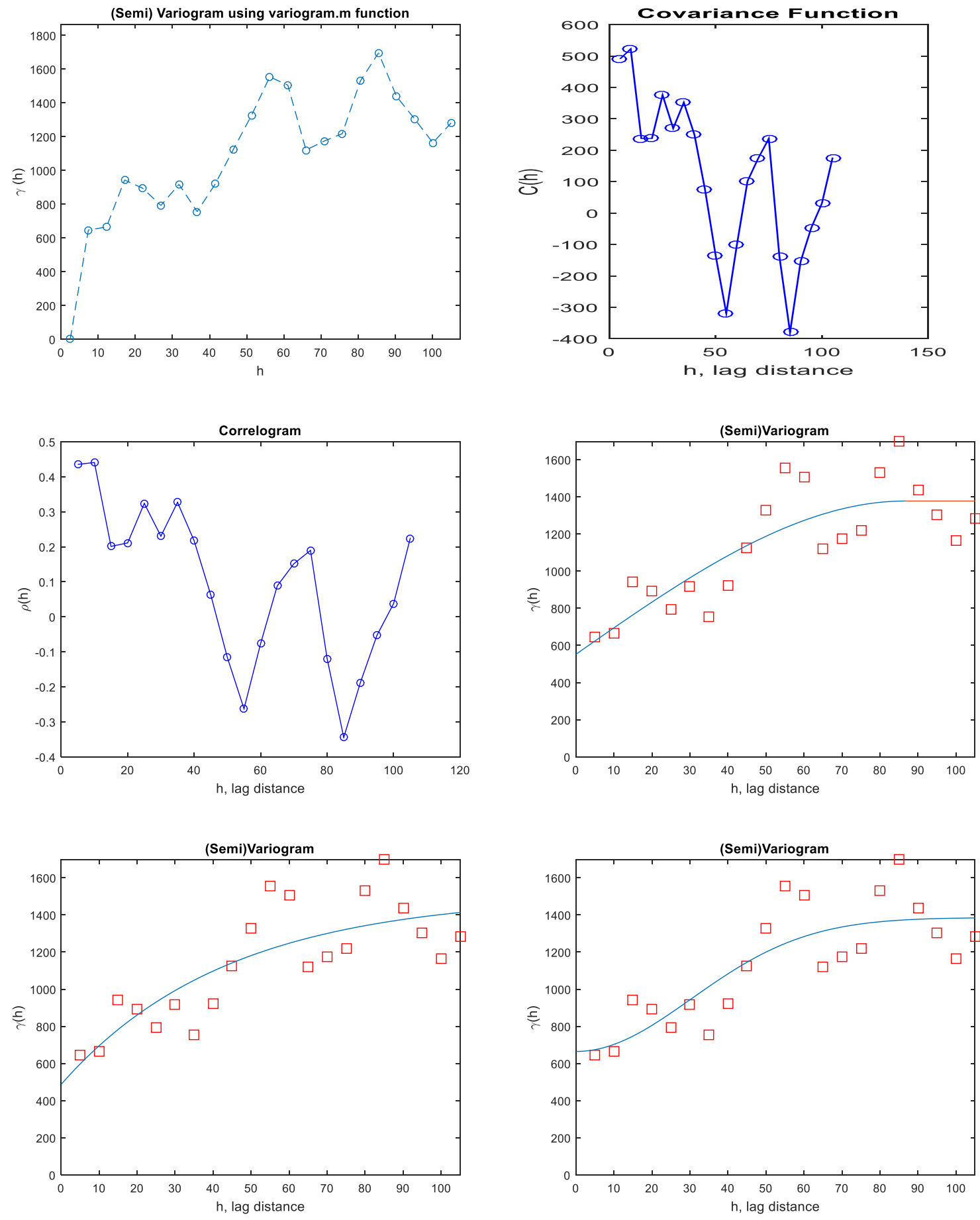


\section{0m Shear wave Velocity without Data after Hill}
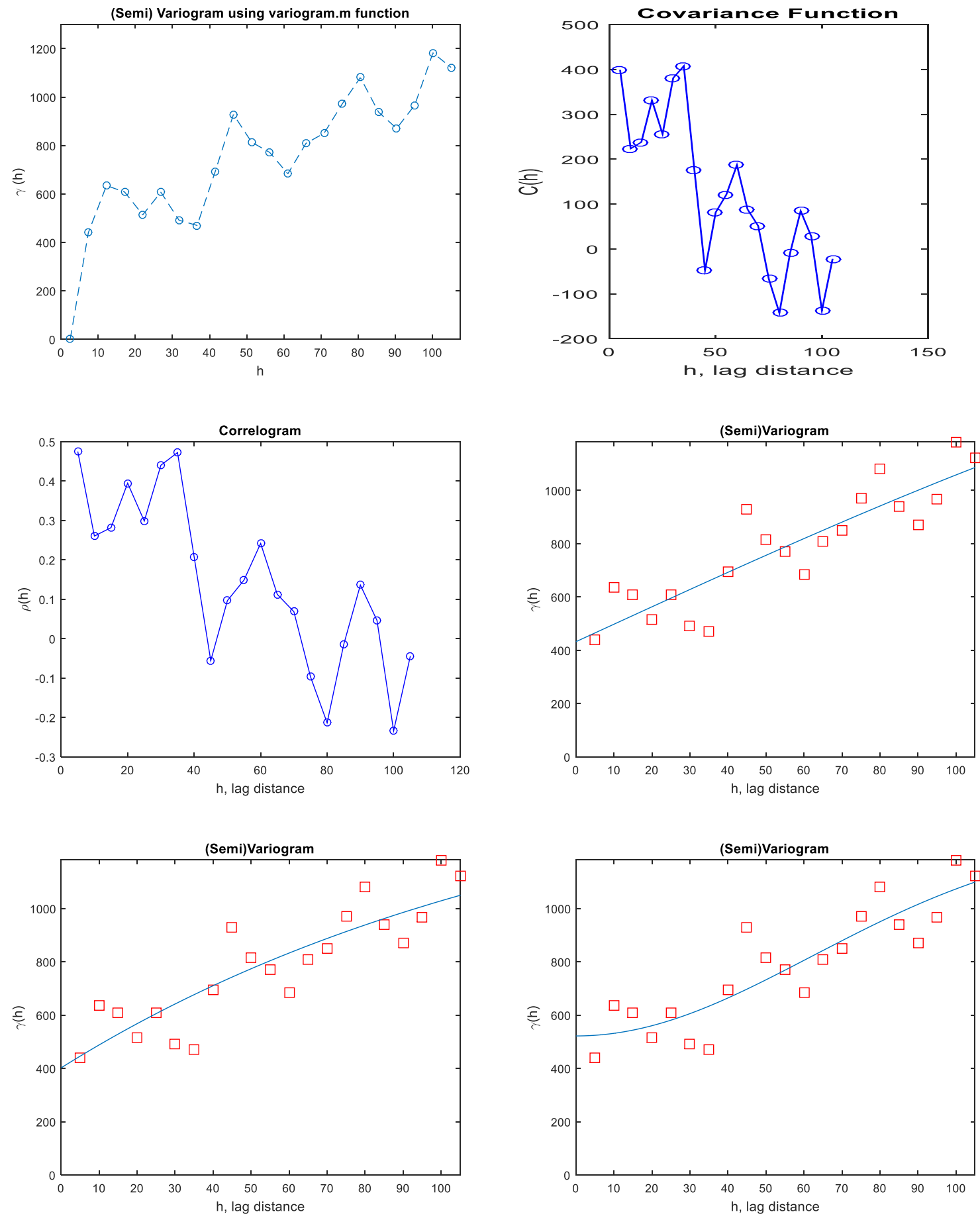


\section{5m Shear wave Velocity without Data after Hill}
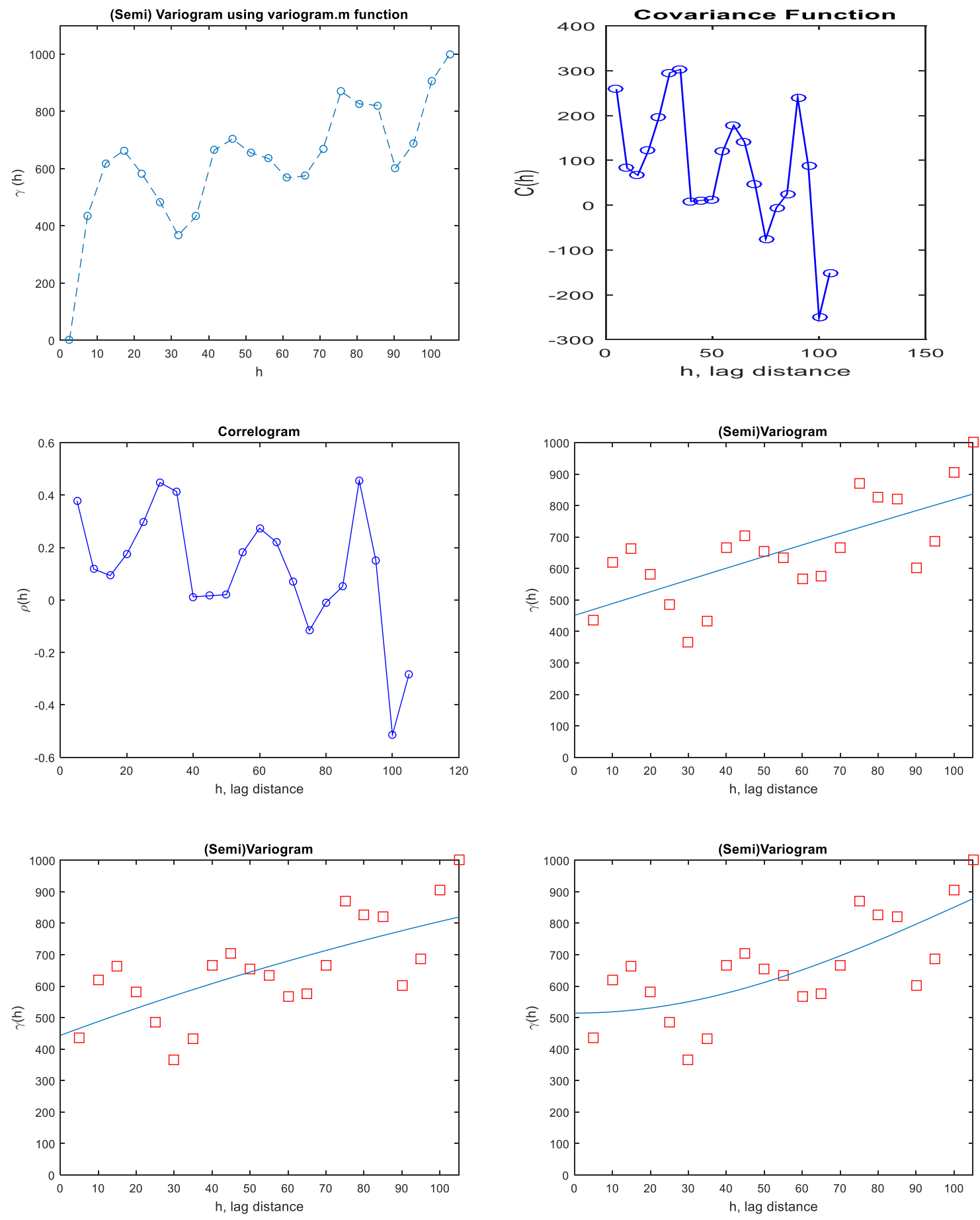
5m Rayleigh wave Velocity with Trend Removed as Linear Function without Data after

Hill
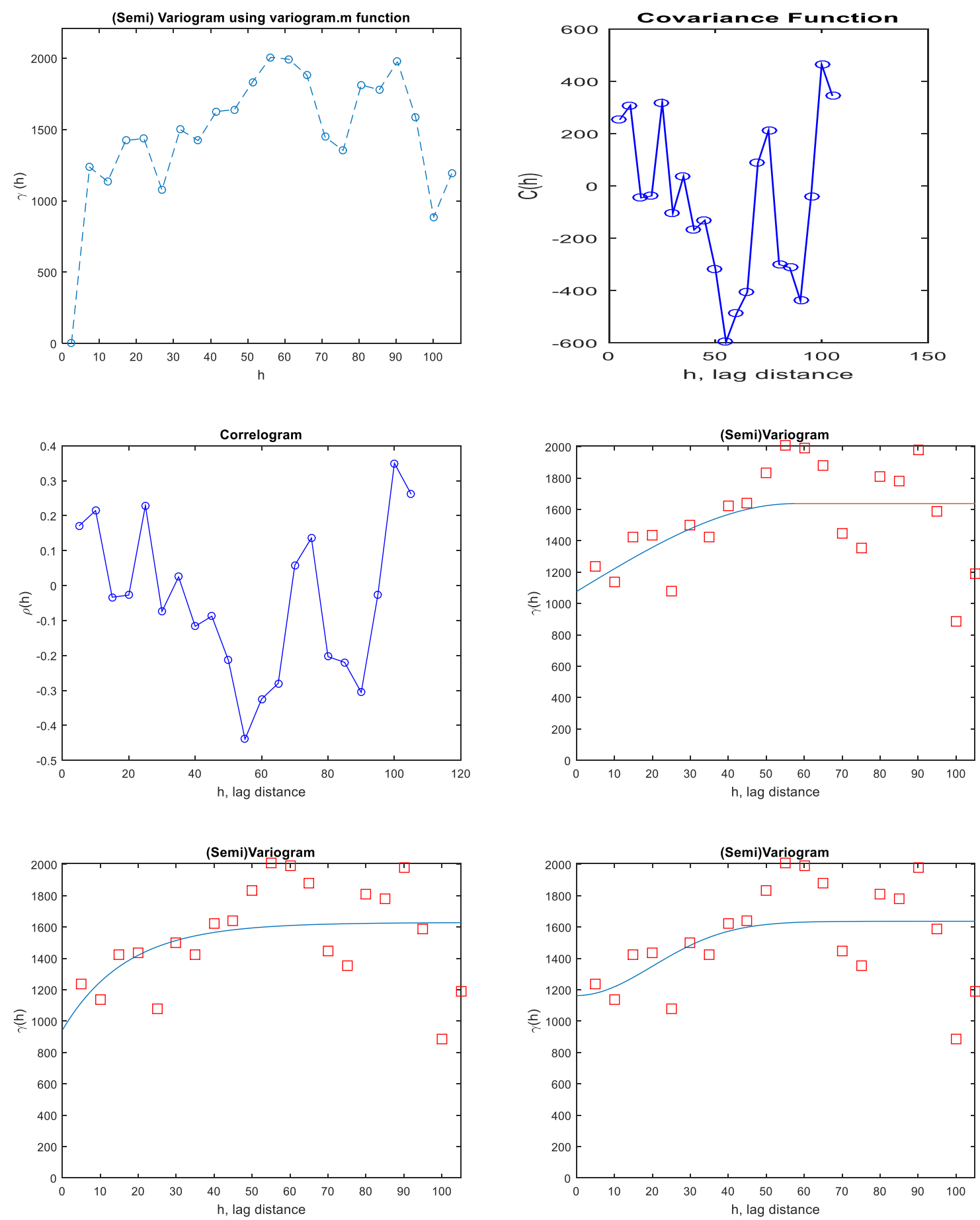
10m Rayleigh wave Velocity with Trend Removed as Linear Function without Data after

Hill
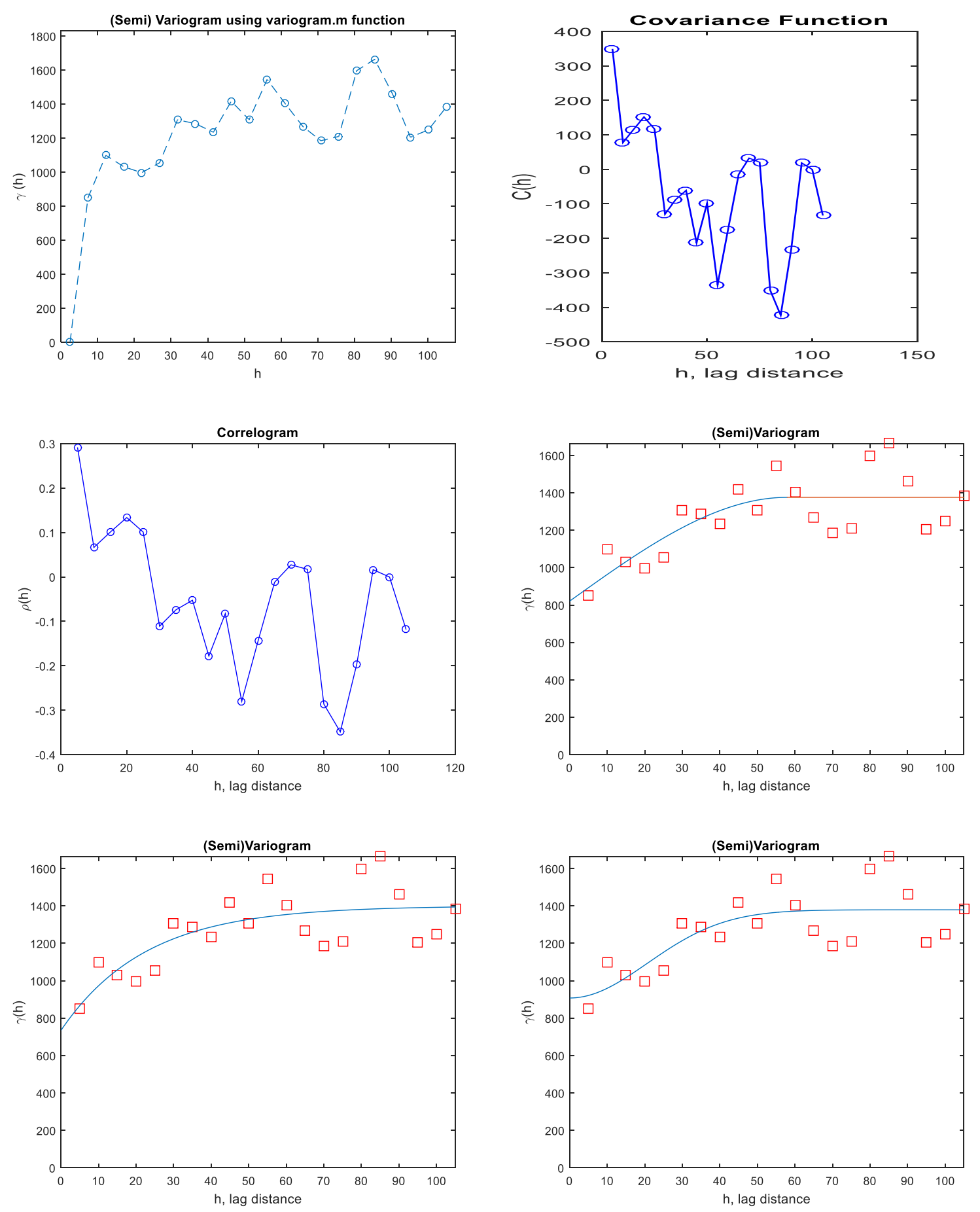


\section{5m Rayleigh wave Velocity with Trend Removed as Linear Function without Data after}

\section{Hill}
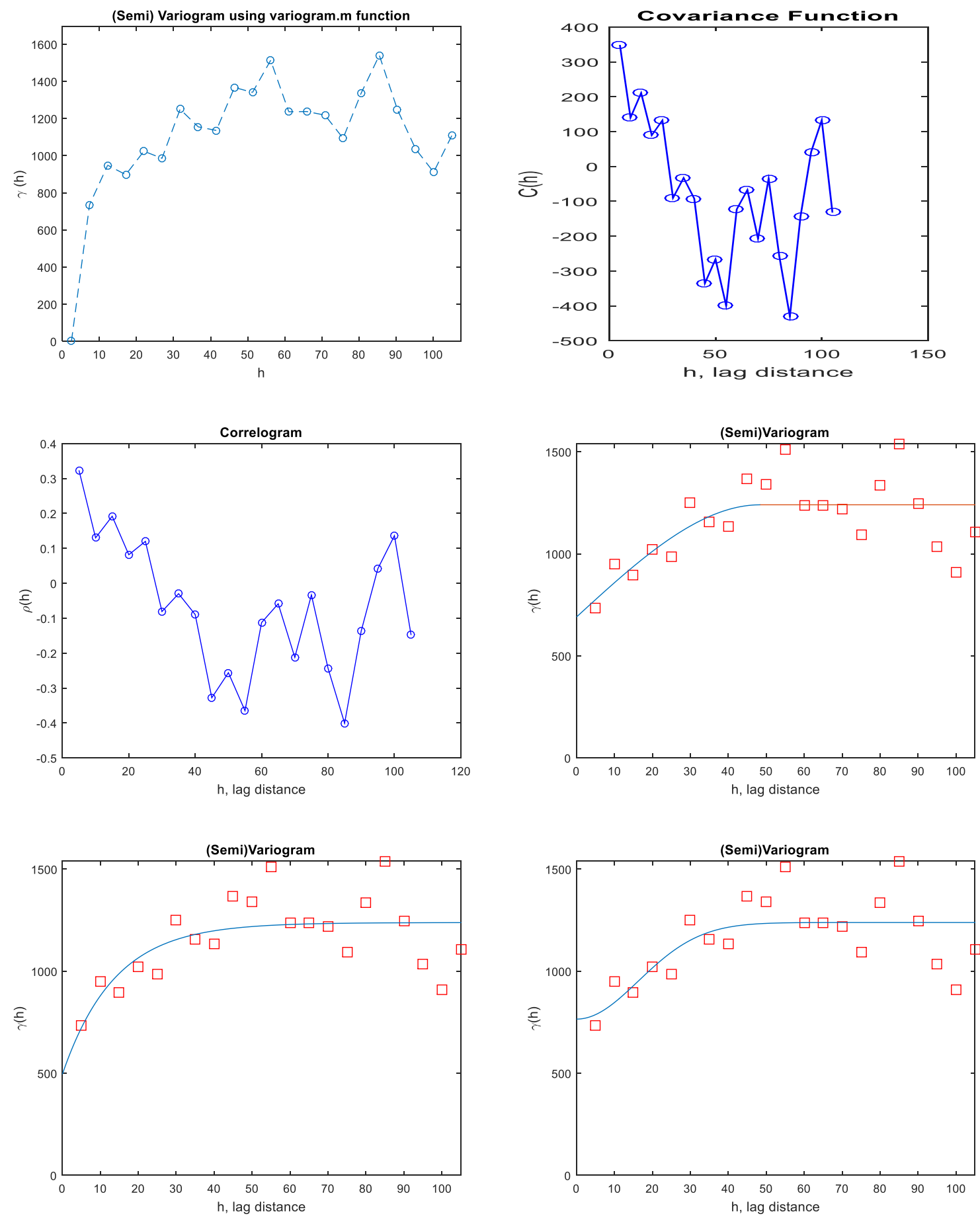
5m Shear wave Velocity with Trend Removed as Linear Function without Data after Hill
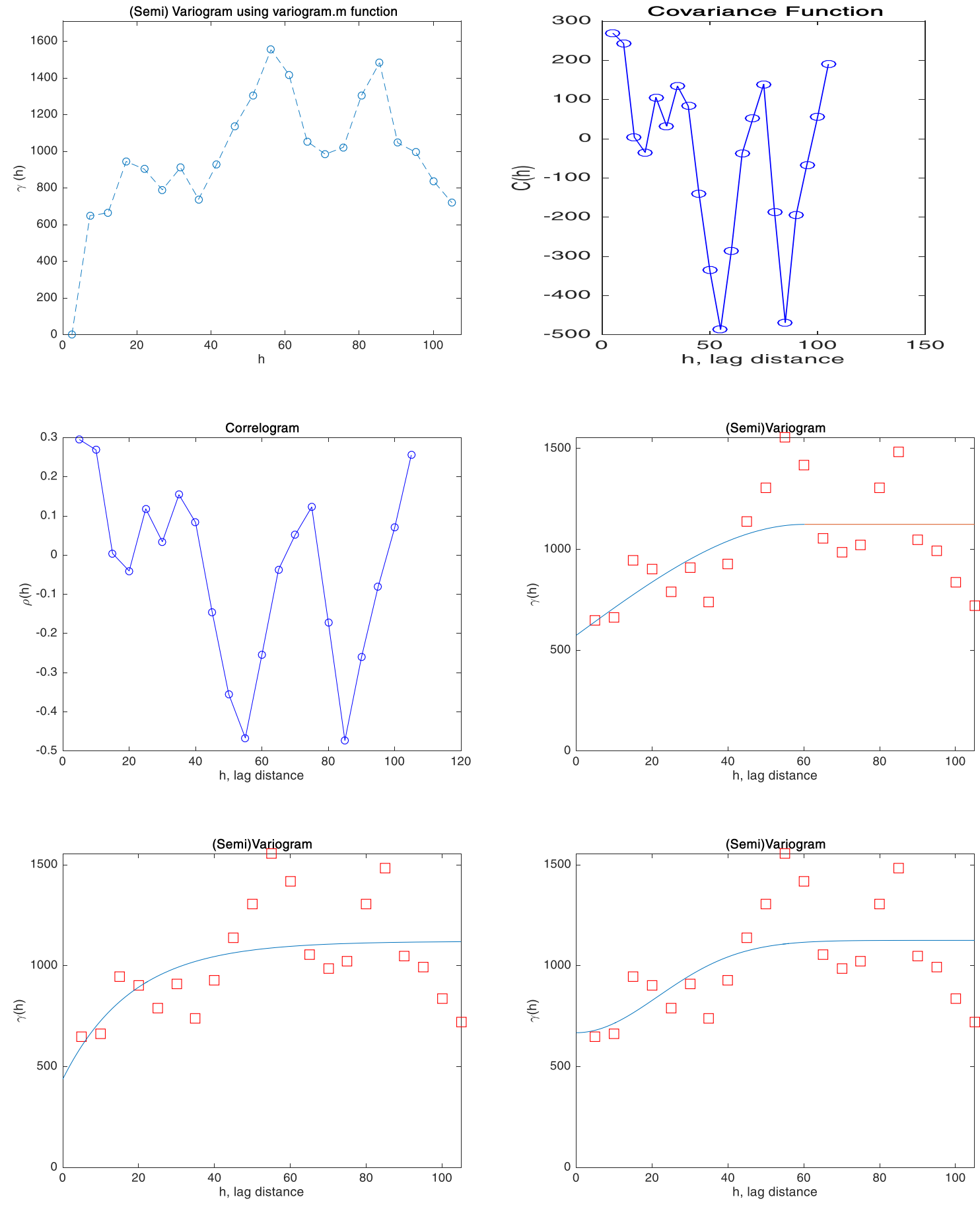
10m Shear wave Velocity with Trend Removed as Linear Function without Data after Hill
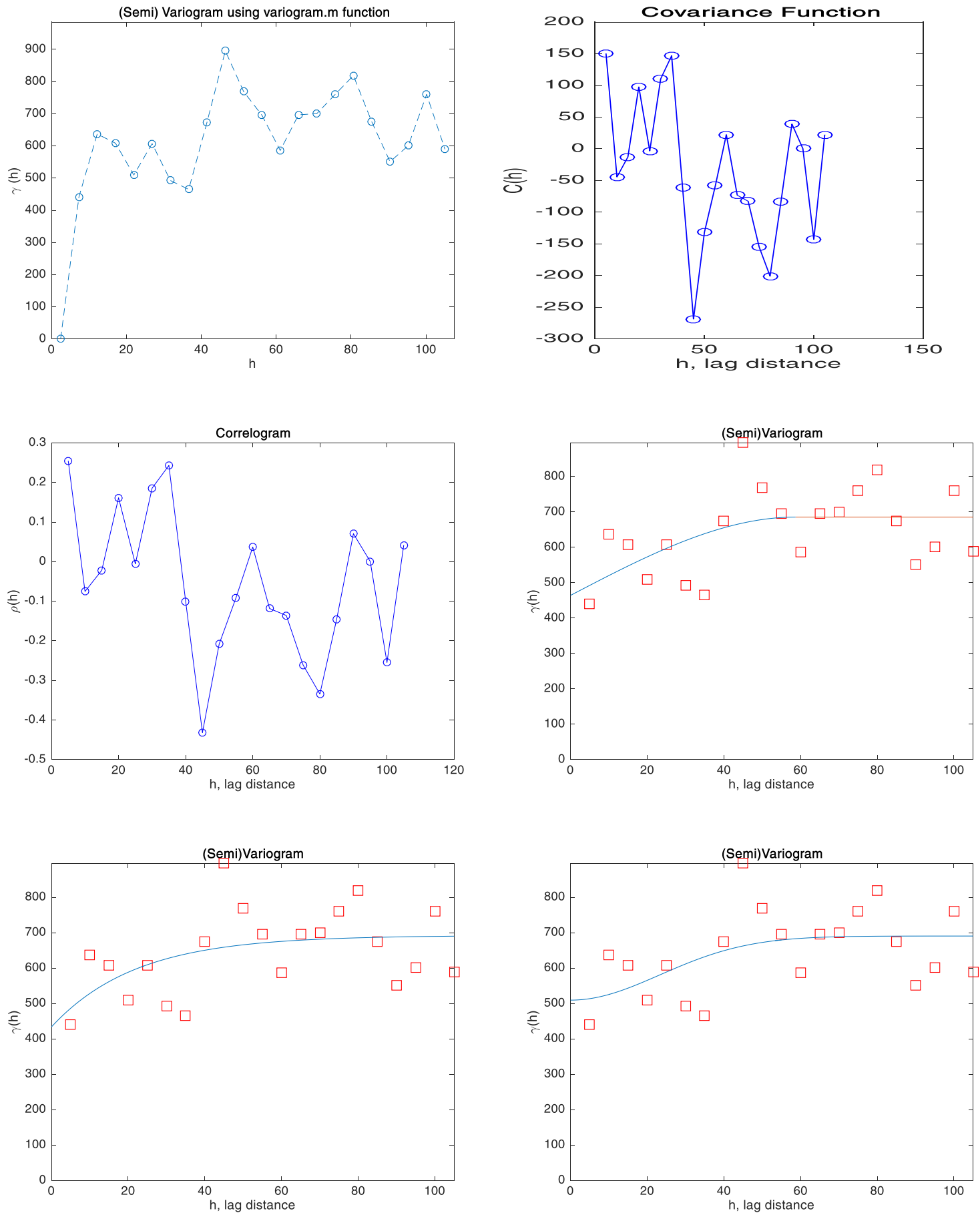
15m Shear wave Velocity with Trend Removed as Linear Function without Data after Hill
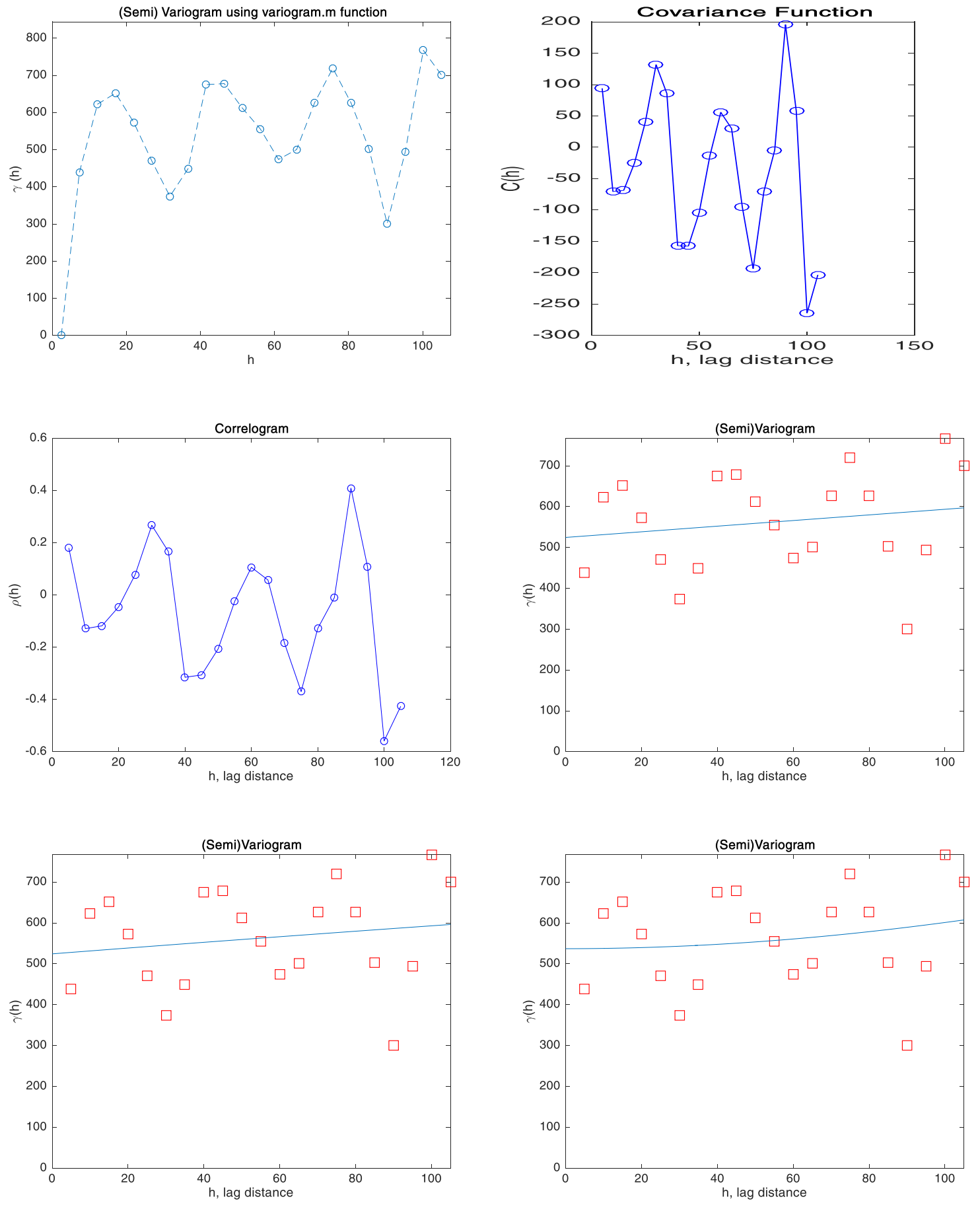


\section{5m Rayleigh wave Velocity with Trend Removed as Linear Piecewise Function}
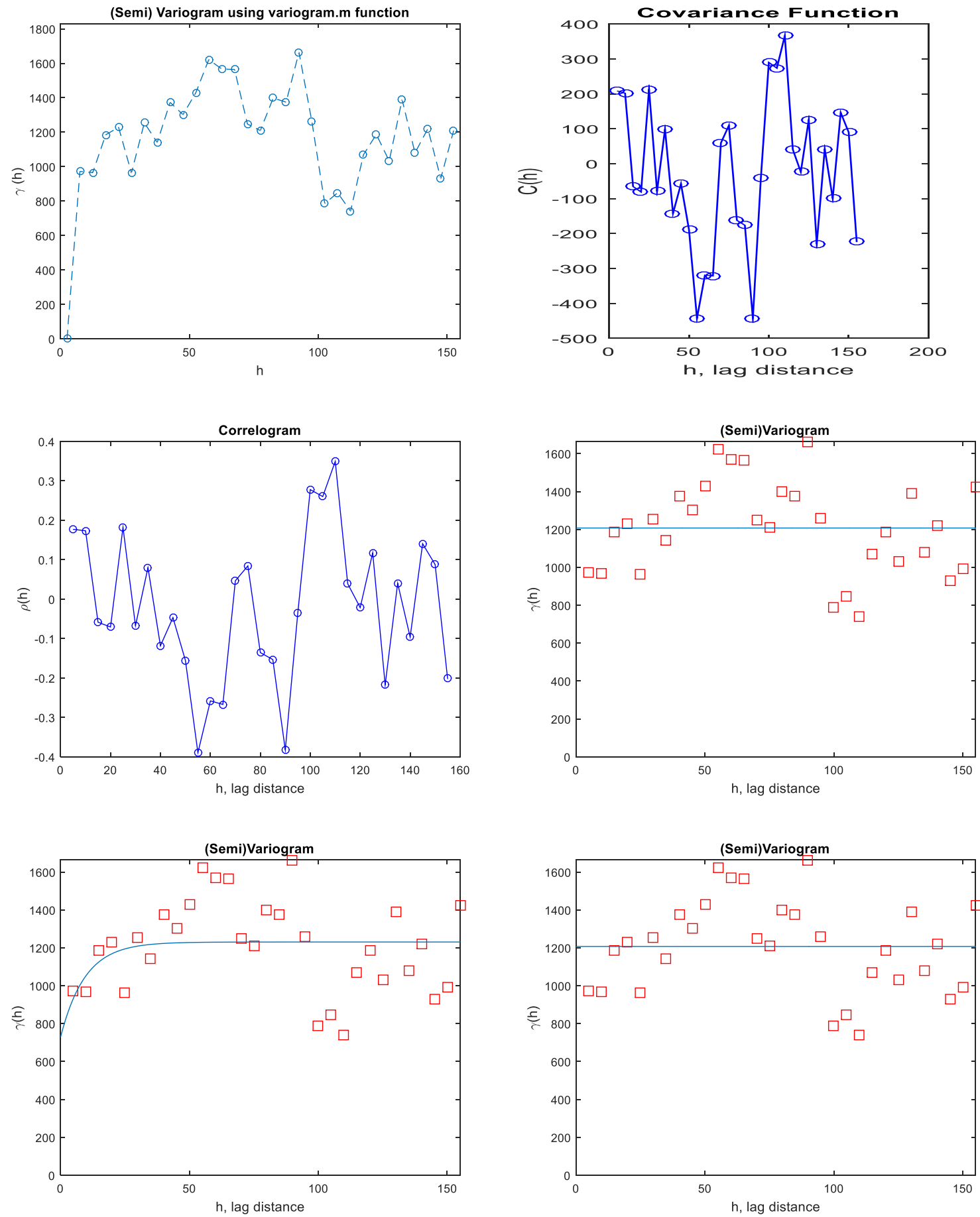


\section{0m Rayleigh wave Velocity with Trend Removed as Linear Piecewise Function}
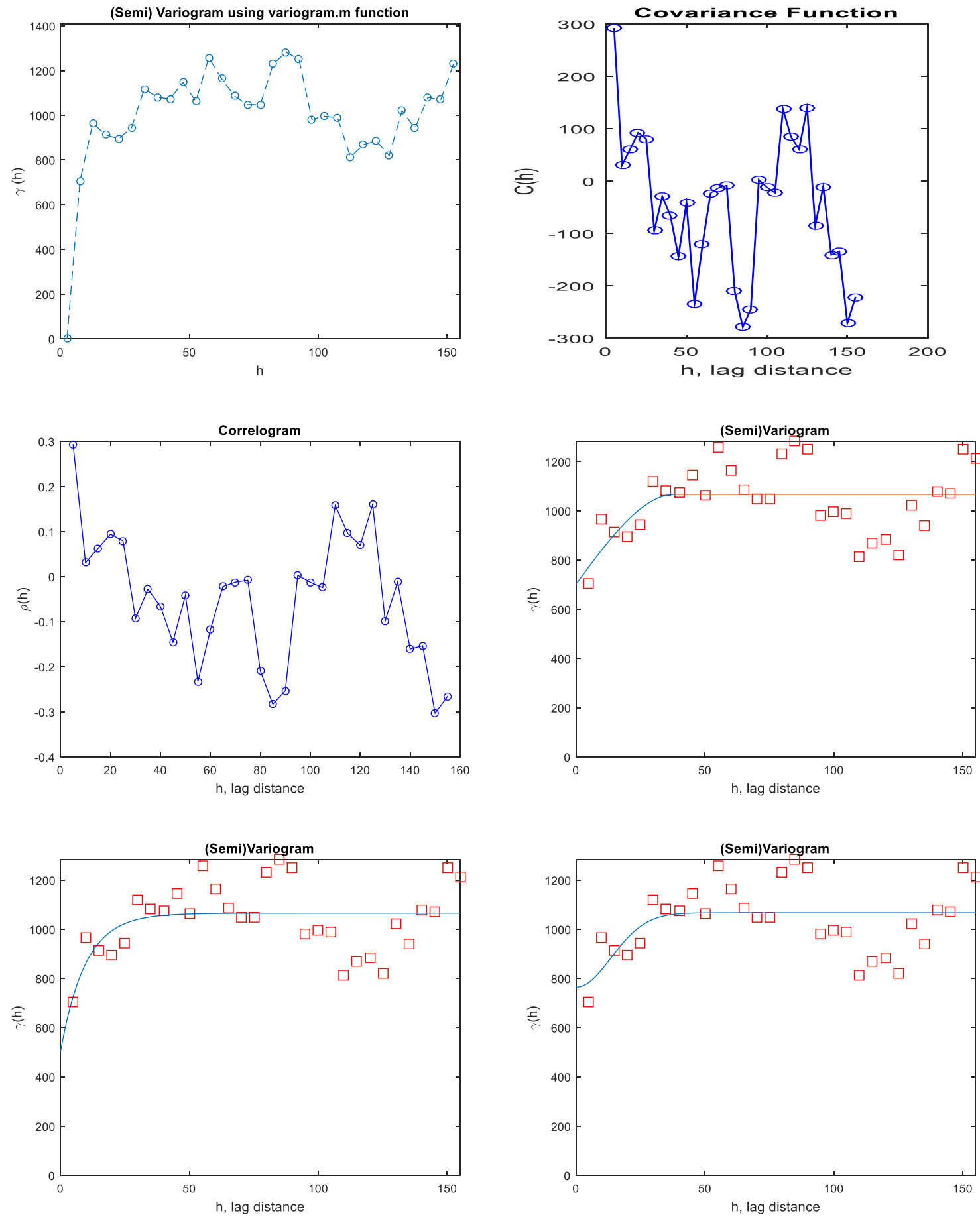


\section{5m Rayleigh wave Velocity with Trend Removed as Linear Piecewise Function}
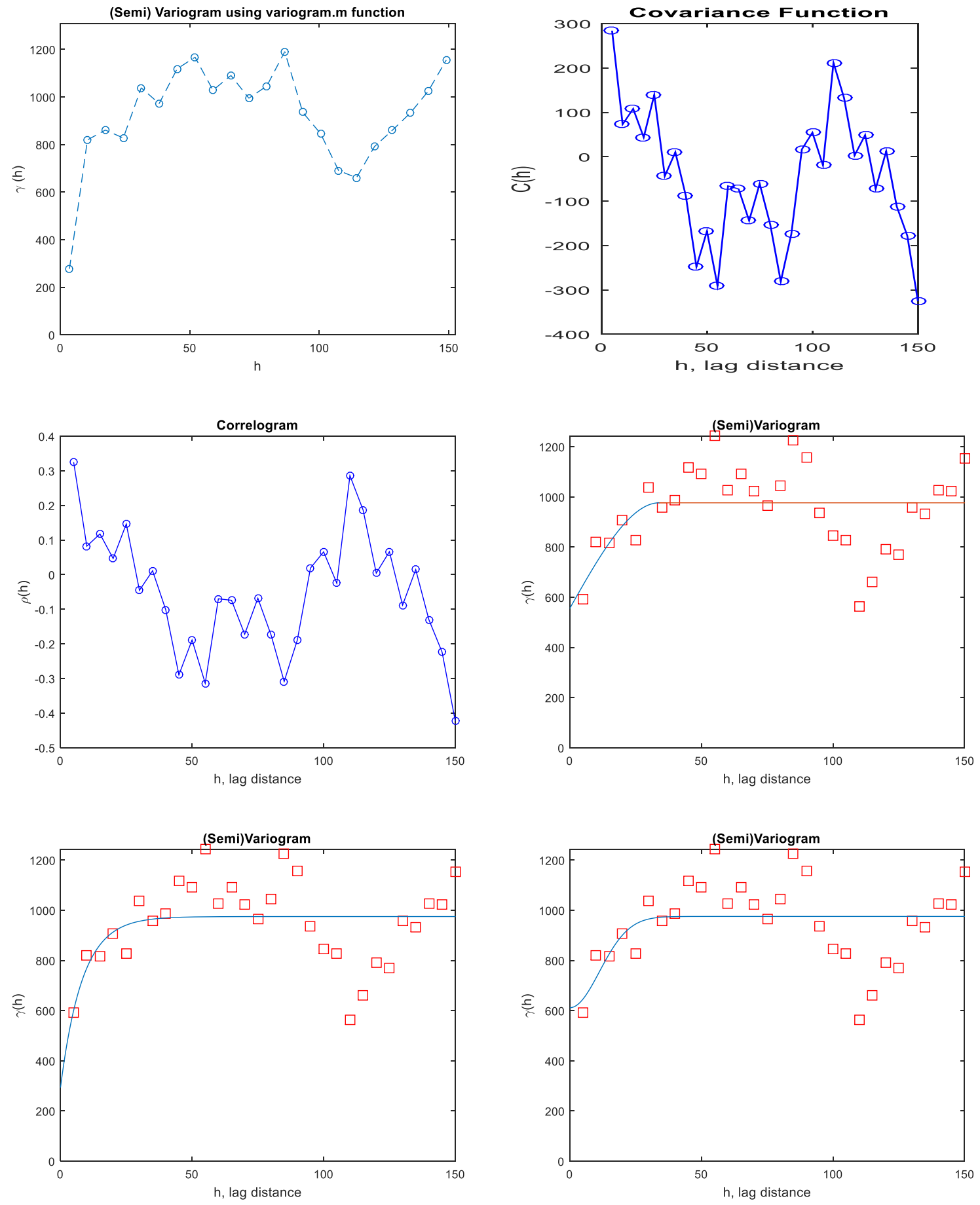


\section{5m Shear wave Velocity with Trend Removed as Linear Piecewise Function}
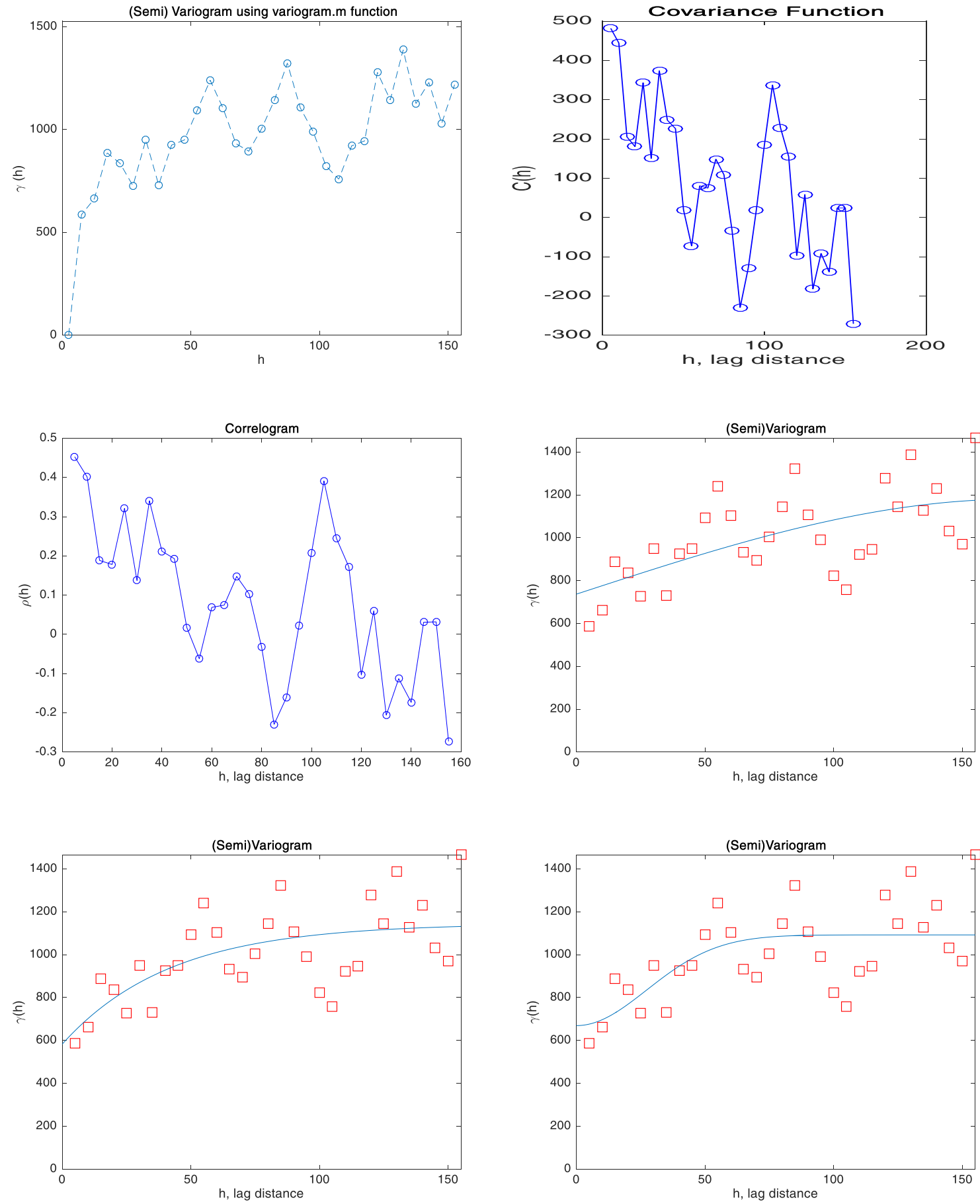


\section{0m Shear wave Velocity with Trend Removed as Linear Piecewise Function}
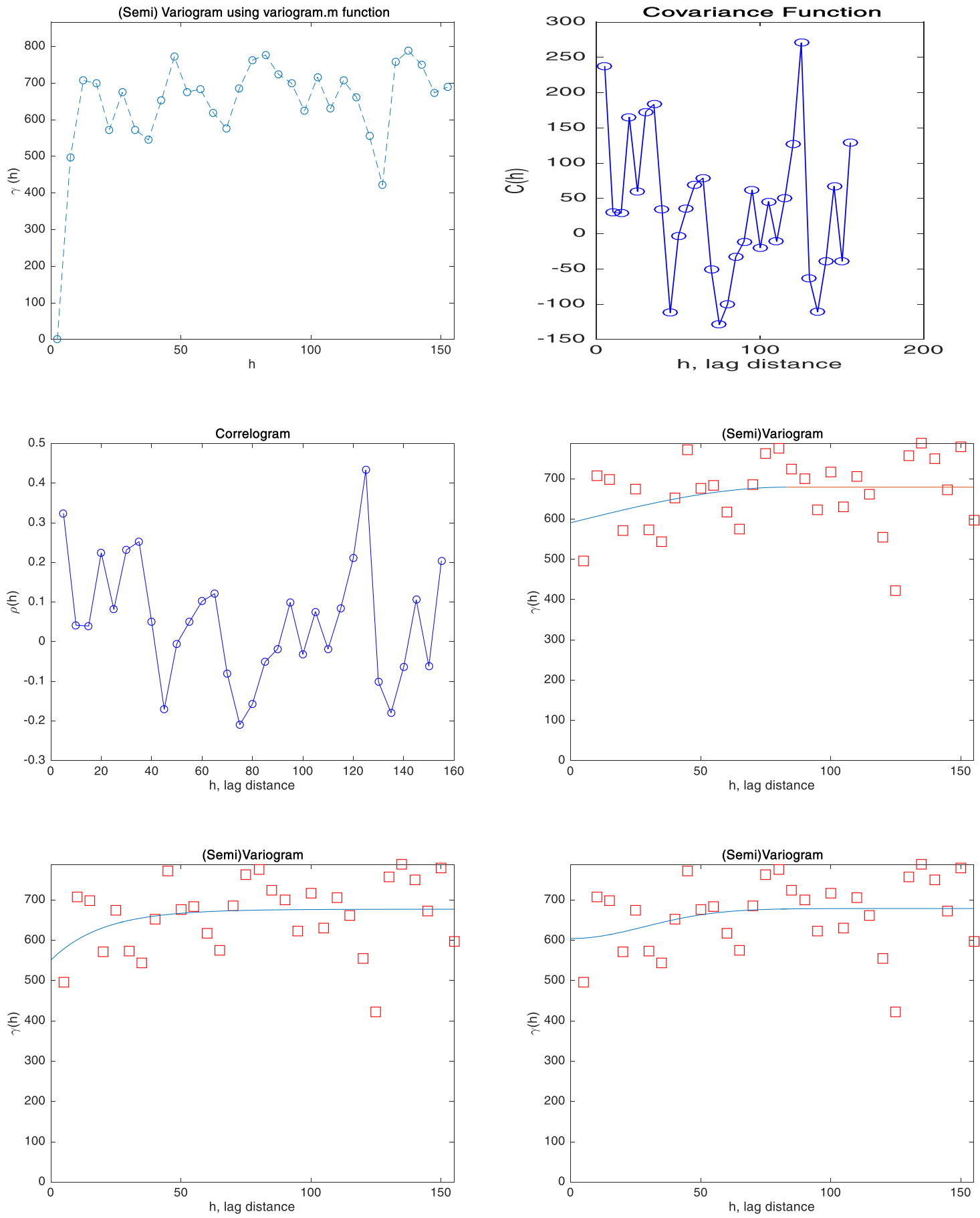


\section{5m Shear wave Velocity with Trend Removed as Linear Piecewise Function}
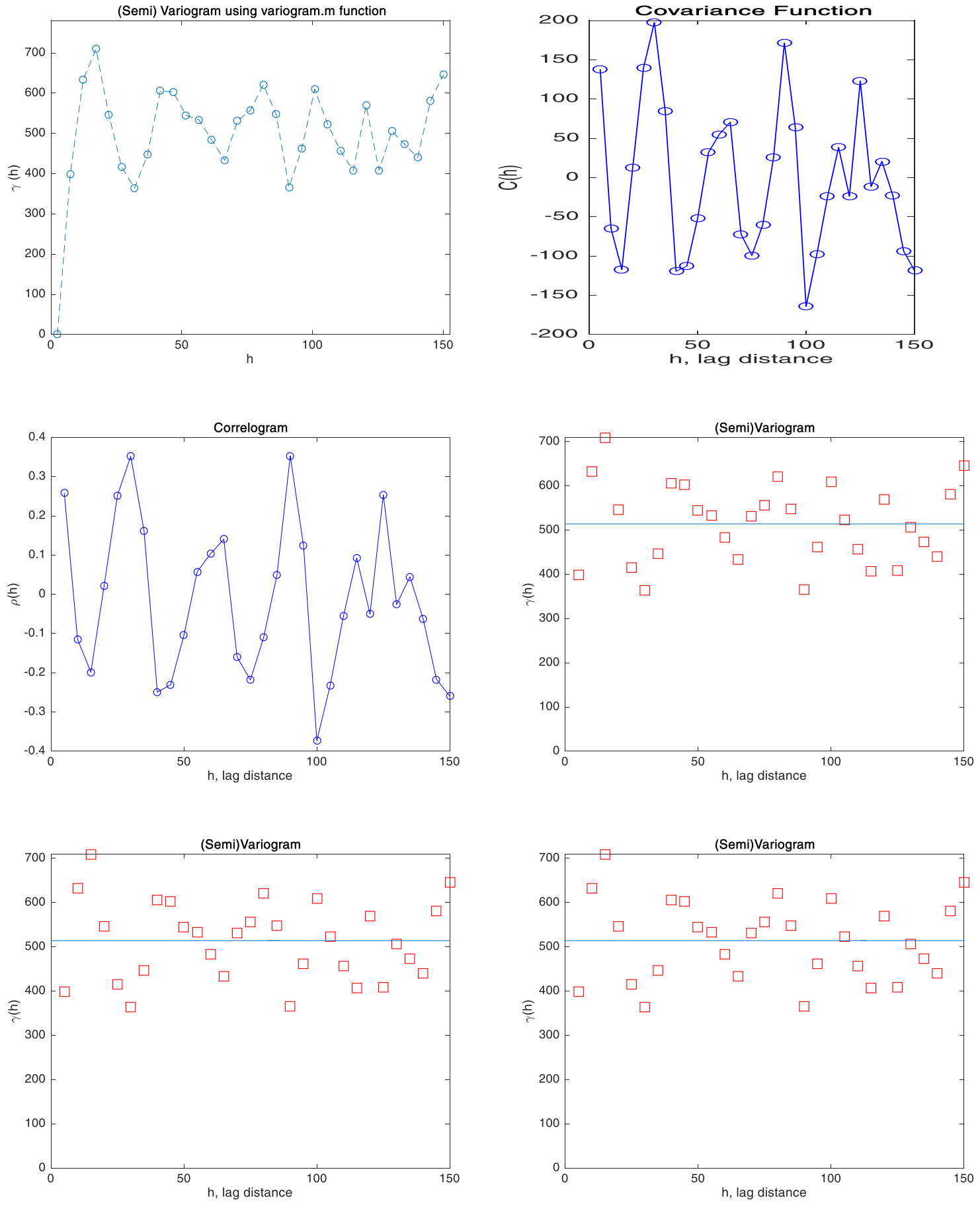
5m Rayleigh wave Velocity with Trend Removed as $4^{\text {th }}$-Order Polynomial Function
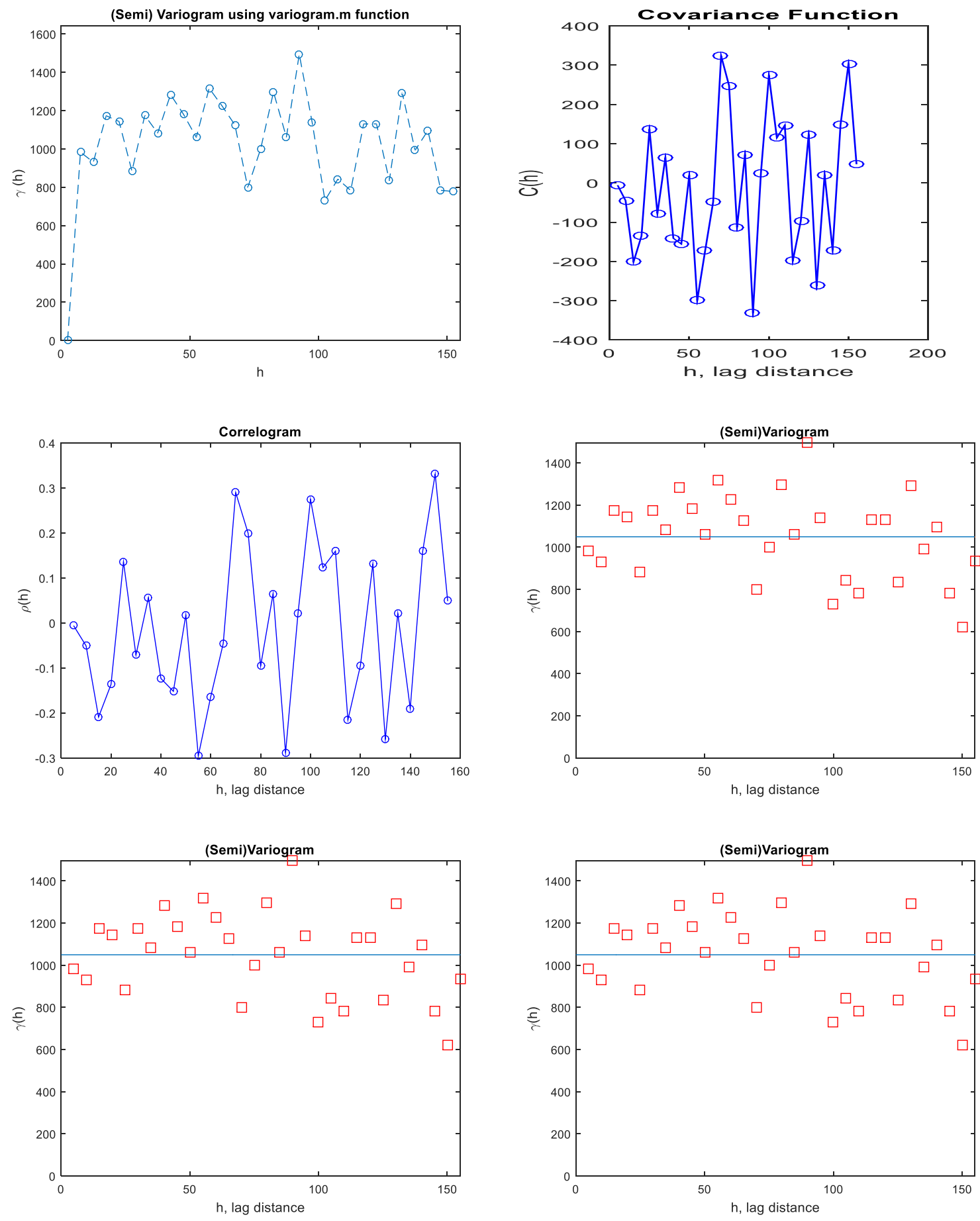
10m Rayleigh wave Velocity with Trend Removed as $4^{\text {th }}$-Order Polynomial Function
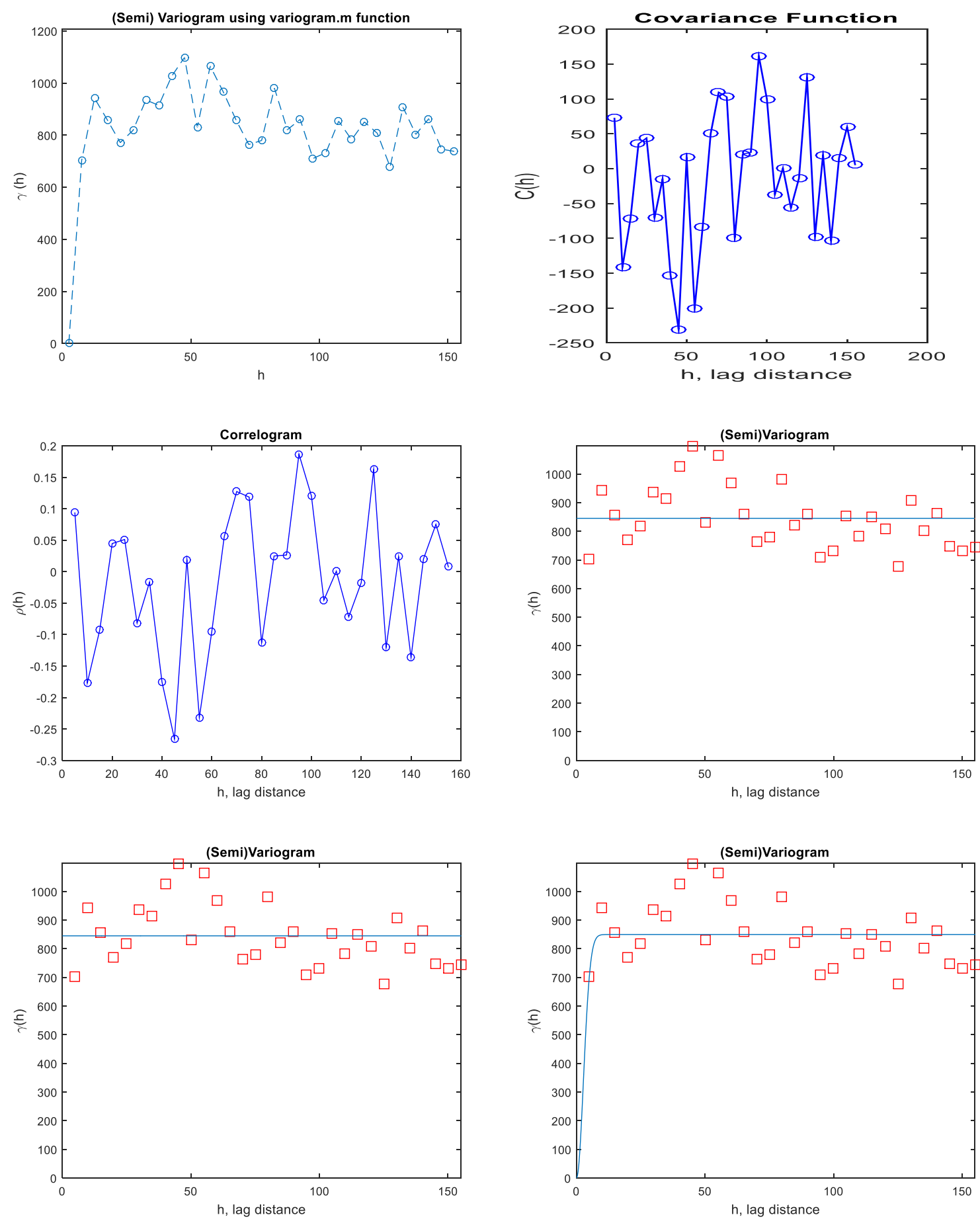
15m Rayleigh wave Velocity with Trend Removed as $4^{\text {th }}$-Order Polynomial Function
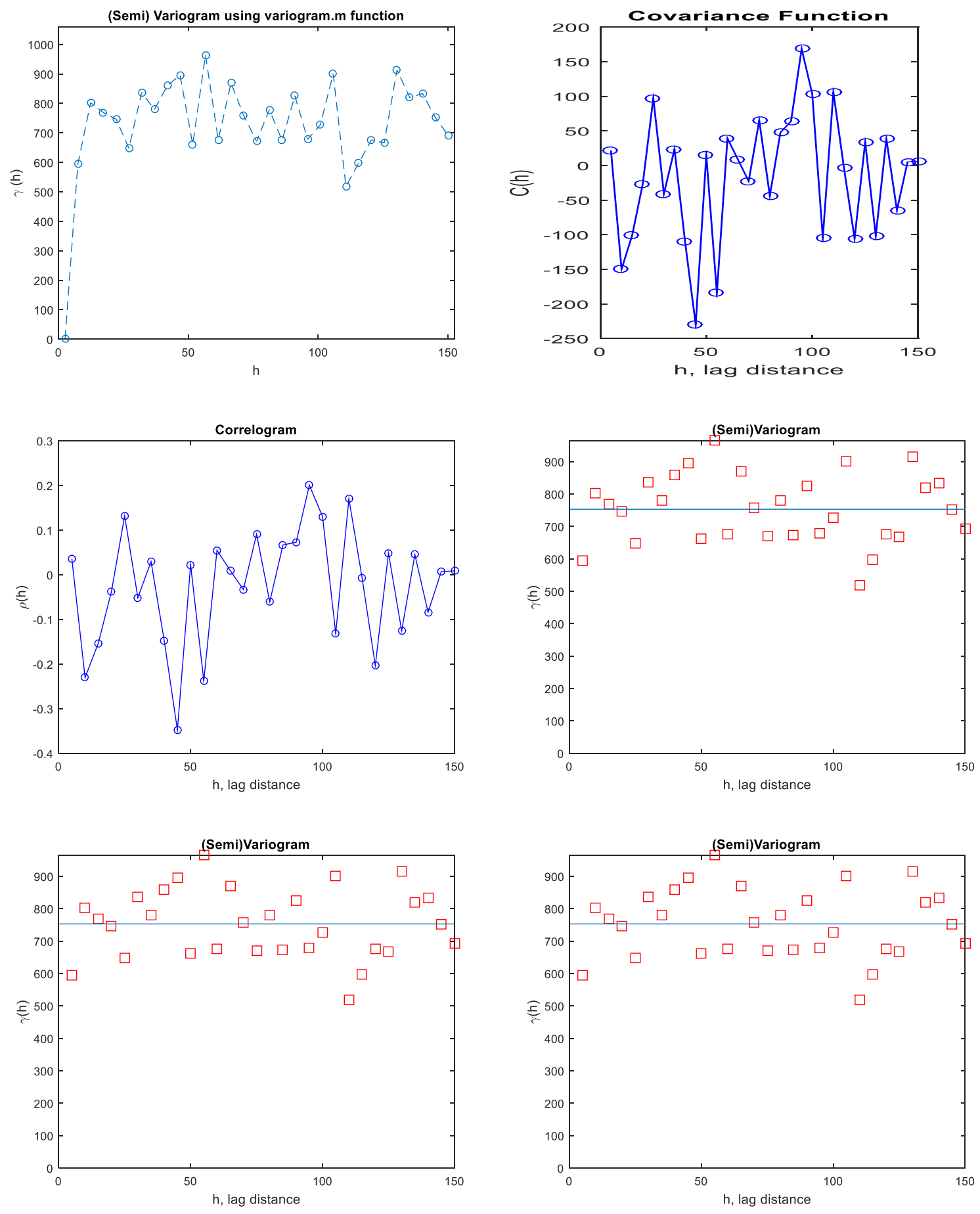
5m Shear wave Velocity with Trend Removed as $4^{\text {th }}$-Order Polynomial Function
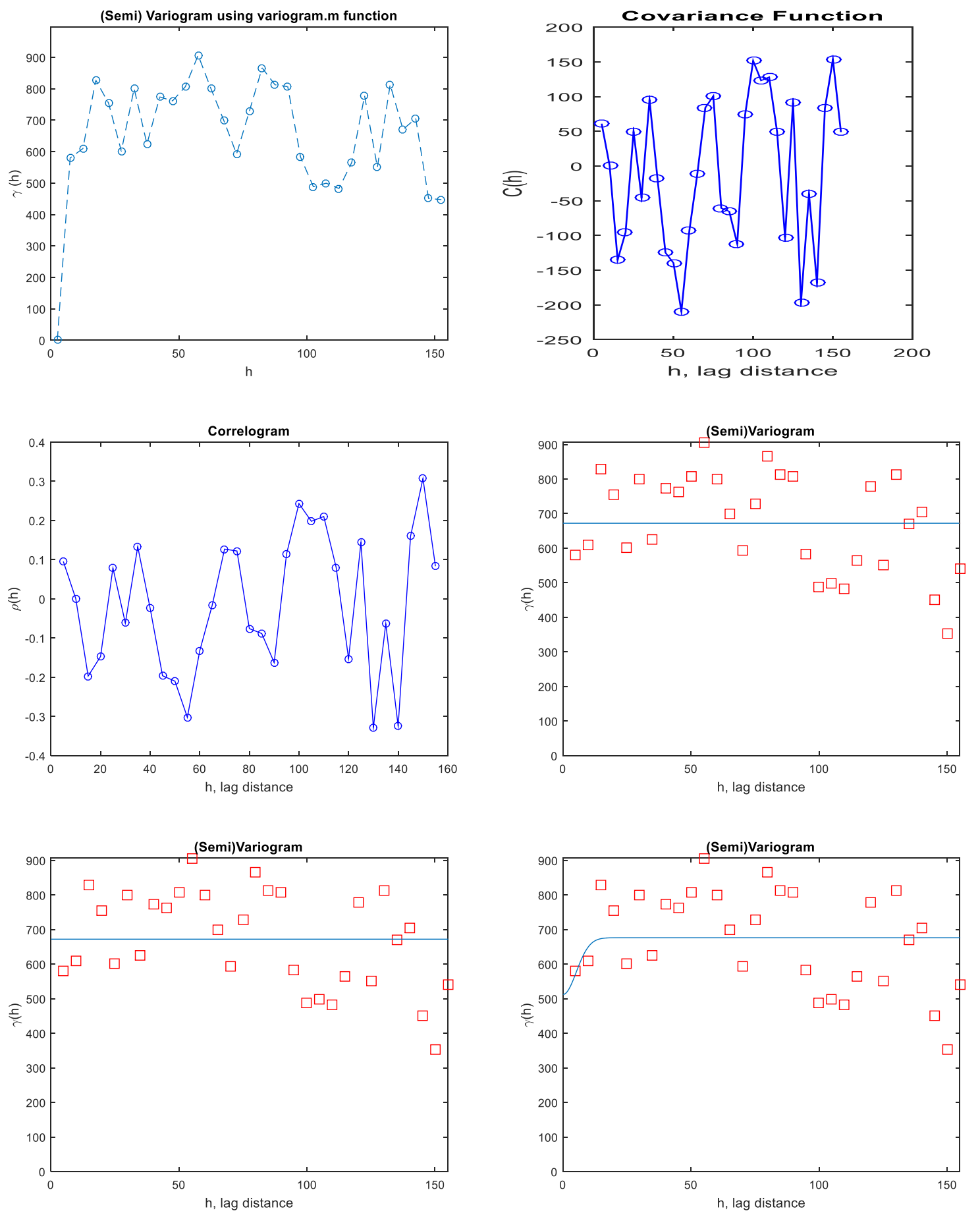
$10 \mathrm{~m}$ Shear wave Velocity with Trend Removed as $4^{\text {th }}$-Order Polynomial Function
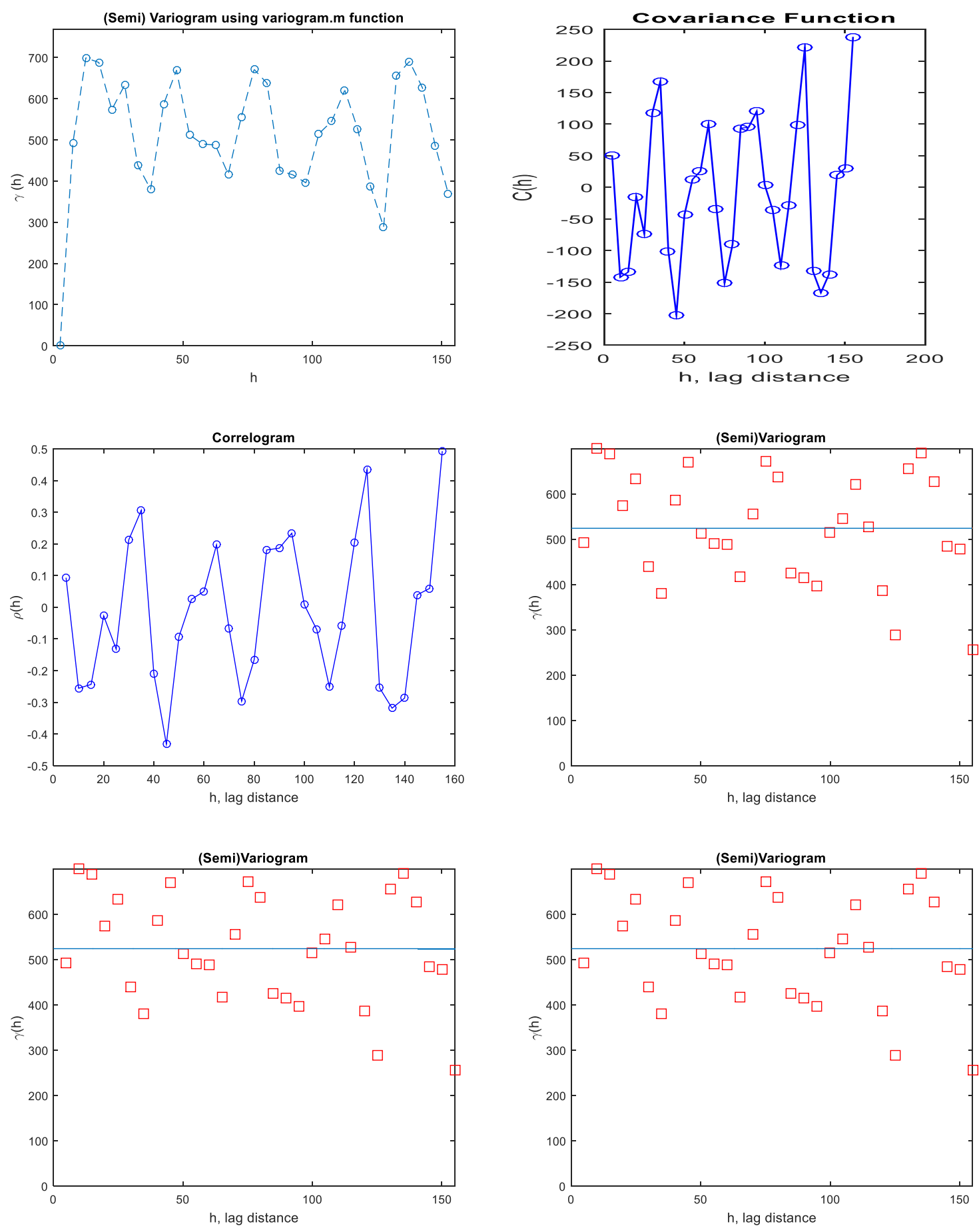
15m Shear wave Velocity with Trend Removed as $4^{\text {th }}$-Order Polynomial Function
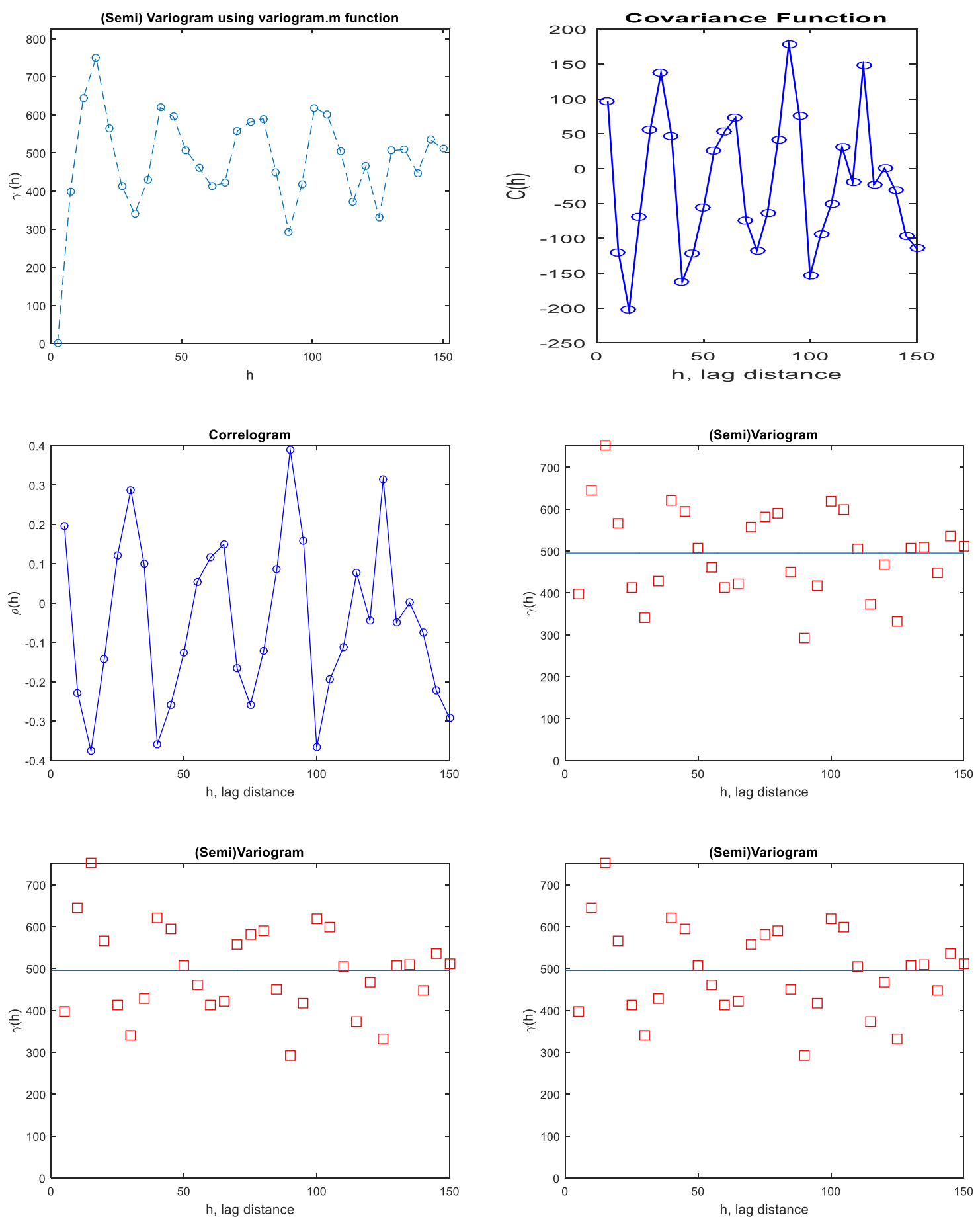
30m Shear wave Velocity based on Boore's 2004 study using 10m Shear wave Velocity
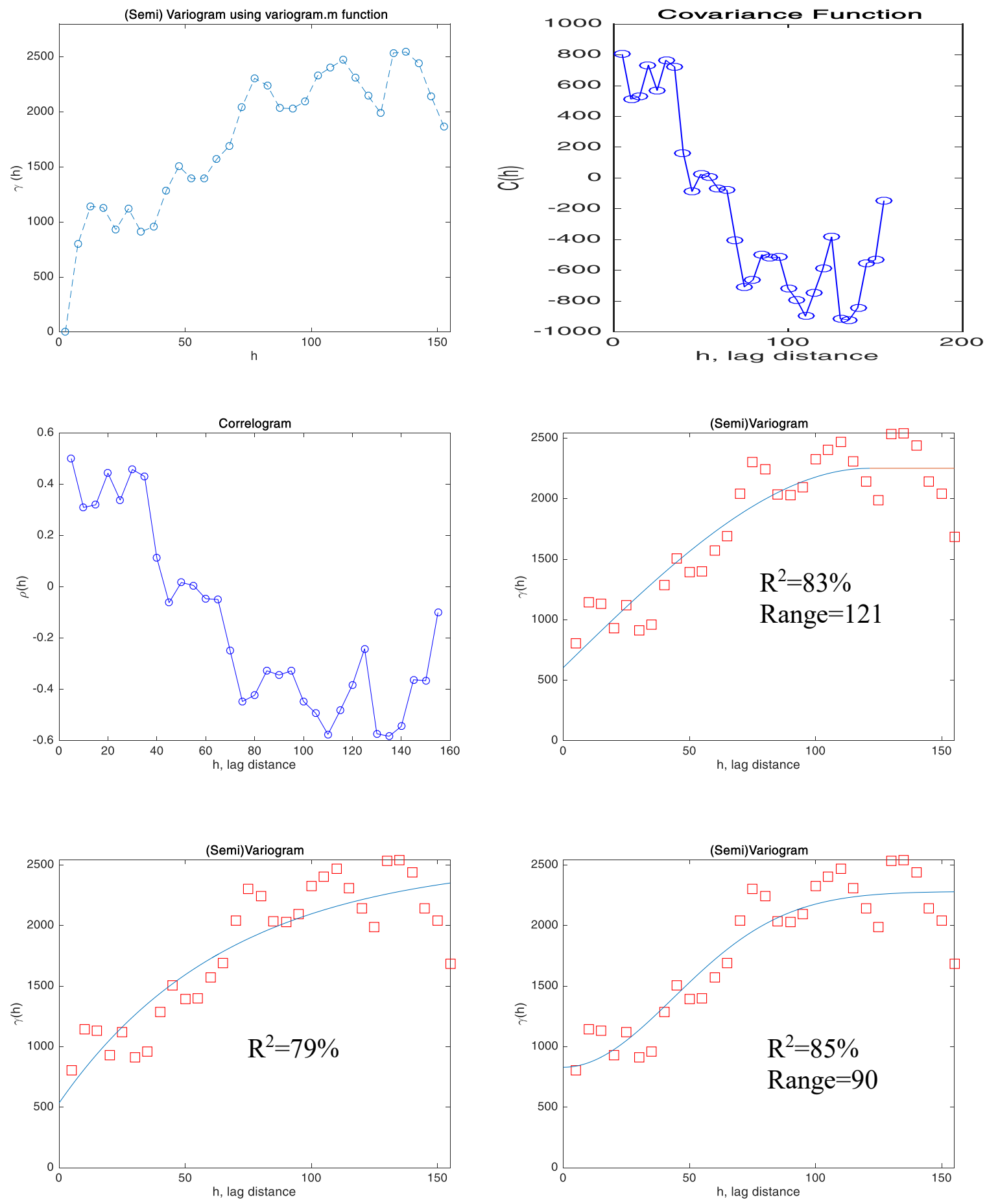


\section{Appendix E: Personal MATLAB Code}

This study used open-source MATLAB code (Schwanghart, 2010) to find and model semi-variograms, but MATLAB code to develop covariance functions and correlograms, verify semi-variogram calculations, and quantify spatial descriptors was written personally and can be referenced below.

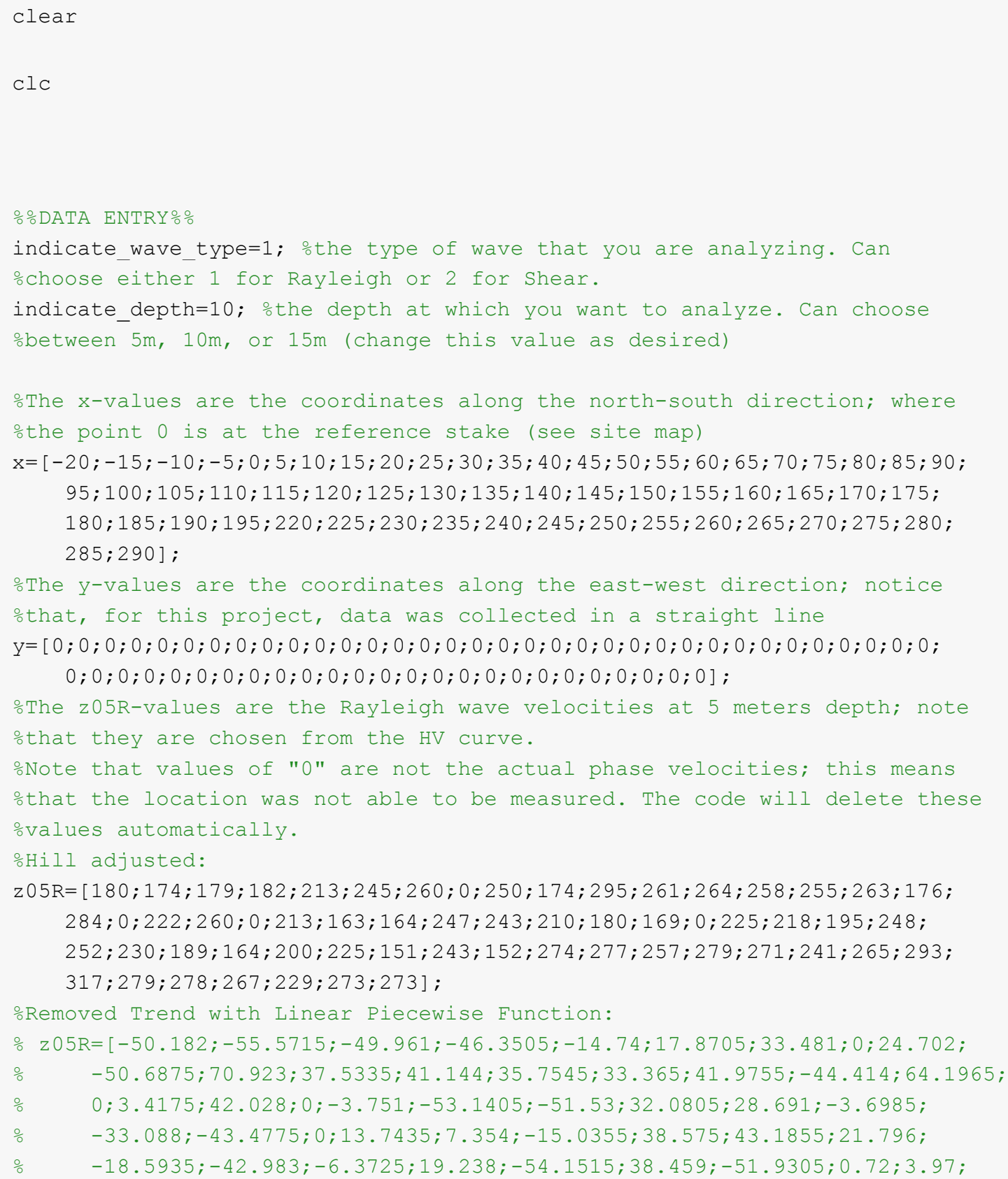


잉 $\quad-15.78 ; 6.47 ;-1.28 ;-31.03 ;-6.78 ; 21.47 ; 45.72 ; 7.97 ; 7.22 ;-3.53 ;-41.28$;

ㅇ. $2.97 ; 3.22]$;

Removed Trend with 4th-Order Polynomial Function:

응 $\mathrm{O} 5 \mathrm{R}=[39.5938991 ; 11.65202707 ;-2.371285314 ;-15.68475721 ; 1.509377006$;

$\quad 22.01536775 ; 27.64395023 ; 0 ; 4.544255082 ;-75.53012822 ; 42.82586886$;

응 $\quad 7.455405425 ; 10.2081254 ; 4.940157474 ; 3.514115128 ; 13.79909663$;

\% $\quad-70.32931498 ; 41.01094813 ; 0 ;-13.34180618 ; 28.76173598 ; 0 ;-9.931306184$;

잉 $\quad-55.8924222 ;-51.02881467 ; 35.59346253 ; 34.91484031 ; 4.882234338$;

응 $\quad-22.5509549 ;-31.42484216 ; 0 ; 27.37725422 ; 21.0054323 ;-1.902698761$;

잉 $\quad 50.64517022 ; 53.64783321 ; 30.11056895 ;-12.954859 ;-40.53020234$;

응 $\quad-7.59072795 ; 13.89478206 ;-64.03596964 ; 23.66120443 ;-71.9630235$;

응 $\quad 24.32227059 ; 22.19689588 ;-2.713601115 ; 14.69333015 ; 2.526724979$;

잉 $\quad-31.09789652 ;-10.05852947 ; 15.7733158 ; 38.53261374 ; 0.360823598$;

\% $\quad 0.405889396 ;-8.177760058 ;-42.22921117 ; 7.418934444 ; 14.94055995]$;

$\circ 10 \mathrm{~m}$ Point values (not averaged):

$\mathrm{z} 10 \mathrm{R}=[325 ; 340 ; 365 ; 320 ; 345 ; 340 ; 345 ; 375 ; 350 ; 365 ; 385 ; 405 ; 380 ; 375 ; 335 ; 345 ; 305$;

응 $\quad 345 ; 350 ; 365 ; 345 ; 300 ; 325 ; 320 ; 330 ; 335 ; 335 ; 300 ; 285 ; 315 ; 330 ; 370 ; 360 ; 280$;

응 $\quad 305 ; 310 ; 300 ; 300 ; 300 ; 330 ; 280 ; 290 ; 320 ; 320 ; 360 ; 365 ; 360 ; 385 ; 360 ; 365 ; 340$;

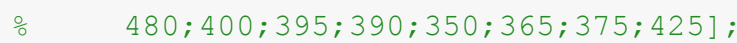

These are the old $10 \mathrm{~m}$ phase velocity values:

$\mathrm{z} 1001 \mathrm{~d}=[325 ; 350 ; 375 ; 355 ; 375 ; 340 ; 360 ; 380 ; 370 ; 355 ; 385 ; 395 ; 405 ; 410 ; 370 ; 370 ; 325 ; 370$;

응 $\quad 365 ; 0 ; 380 ; 280 ; 325 ; 345 ; 345 ; 355 ; 350 ; 310 ; 300 ; 325 ; 310 ; 325 ; 360 ; 275 ; 325 ; 330$;

\% $\quad 315 ; 320 ; 305 ; 285 ; 275 ; 300 ; 340 ; 320 ; 365 ; 370 ; 350 ; 375 ; 380 ; 385 ; 320 ; 395 ; 415 ; 0$;

응 $\quad 365 ; 335 ; 365 ; 365 ; 310]$;

The z05S-values are the Shear wave velocities at 5 meters depth; they are opulled from the shear wave velocity model given after inversion.

oNote that values of "O" are not the actual phase velocities; this means

othat the location was not able to be measured. The code will delete these ○values automatically.

ㅇill adjusted:

$\mathrm{z} 05 \mathrm{~S}=[193 ; 211 ; 236 ; 234 ; 255 ; 259 ; 259 ; 0 ; 248 ; 240 ; 293 ; 295 ; 298 ; 293 ; 266 ; 262 ; 190 ;$

$324 ; 0 ; 254 ; 268 ; 0 ; 238 ; 209 ; 214 ; 269 ; 255 ; 230 ; 188 ; 209 ; 0 ; 245 ; 249 ; 239 ;$

$240 ; 232 ; 220 ; 192 ; 193 ; 206 ; 234 ; 178 ; 232 ; 189 ; 301 ; 313 ; 287 ; 299 ; 325 ; 301 ; 286$;

$359 ; 361 ; 308 ; 298 ; 301 ; 286 ; 293 ; 309]$;

\%Non-hill adjusted:

zㅡㅇ $\mathrm{z} 5 \mathrm{~S}=[193 ; 211 ; 236 ; 234 ; 255 ; 259 ; 259 ; 0 ; 248 ; 240 ; 293 ; 295 ; 298 ; 293 ; 266 ; 262 ; 190$;

잉 $\quad 324 ; 0 ; 254 ; 268 ; 0 ; 238 ; 209 ; 214 ; 269 ; 255 ; 230 ; 188 ; 209 ; 0 ; 245 ; 249 ; 239$;

응 $\quad 240 ; 232 ; 220 ; 192 ; 193 ; 206 ; 234 ; 178 ; 232 ; 189 ; 255 ; 270 ; 255 ; 307 ; 282 ; 257 ; 280$;

잉 $299 ; 332 ; 265 ; 250 ; 264 ; 244 ; 261 ; 259]$;

으omoved Trend with Linear Piecewise Function:

$z 05 S=[-69.178 ;-50.0885 ;-23.999 ;-24.9095 ;-2.82 ; 2.2695 ; 3.359 ; 0 ;-5.462 ;-12.3725$

ㄴ $41.717 ; 44.8065 ; 48.896 ; 44.9855 ; 19.075 ; 16.1645 ;-54.746 ; 80.3435 ; 0 ; 12.5225$;

$27.612 ; 0 ;-0.209 ;-28.1195 ;-22.03 ; 34.0595 ; 21.149 ;-2.7615 ;-43.672 ;-21.5825 ; 0$;

응 $16.5965 ; 21.686 ; 12.7755 ; 14.865 ; 7.9545 ;-2.956 ;-29.8665 ;-27.777 ;-13.6875$;

$15.402 ;-39.5085 ; 15.581 ;-26.3295 ;-54.782 ;-39.5925 ;-54.403 ;-2.2135 ;-27.024$;

$-51.8345 ;-28.645 ;-9.4555 ; 23.734 ;-43.0765 ;-57.887 ;-43.6975 ;-63.508 ;-46.3185$;

ㅇ -48.129$]$;

\%Removed Trend with 4th-Order Polynomial Function: 


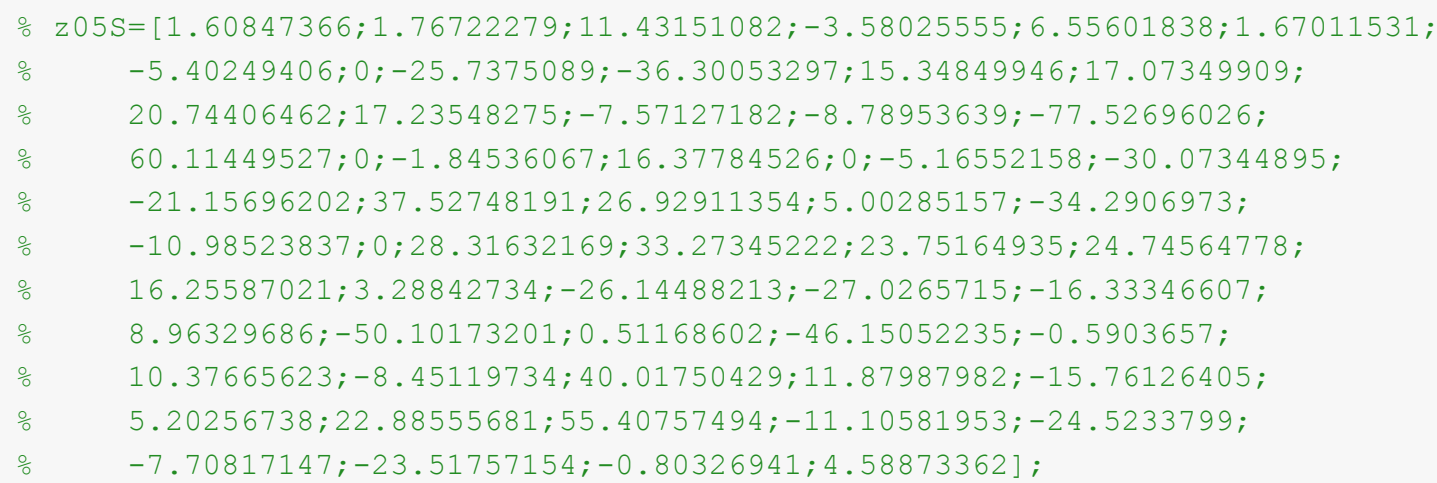

oThe z12R-values are the Rayleigh wave velocities at 12 meters depth; note othat they are just "eye-balled" values from the HV curve.

oNote that values of "O" are not the actual phase velocities; this means othat the location was not able to be measured. The code will delete these values automatically.

№n-Hill adjusted:

$\mathrm{z} 10 \mathrm{R}=[210 ; 210 ; 226 ; 224 ; 249 ; 263 ; 280 ; 332 ; 275 ; 222 ; 309 ; 297 ; 293 ; 294 ; 282 ; 280 ; 208$; $313 ; 305 ; 262 ; 284 ; 290 ; 243 ; 202 ; 209 ; 265 ; 258 ; 231 ; 210 ; 210 ; 303 ; 255 ; 251 ; 225 ;$ $256 ; 253 ; 242 ; 213 ; 197 ; 230 ; 243 ; 182 ; 253 ; 188 ; 294 ; 299 ; 286 ; 310 ; 302 ; 275 ; 283$; $332 ; 341 ; 305 ; 302 ; 289 ; 265 ; 298 ; 299]$;

․Removed Trend with Linear Piecewise Function

ㄱ10R $=[-61.77 ;-60.8 ;-43.83 ;-44.86 ;-18.89 ;-3.92 ; 14.05 ; 67.02 ; 10.99 ;-41.04$;

응 $\quad 46.93 ; 35.9 ; 32.87 ; 34.84 ; 23.81 ; 22.78 ;-48.25 ; 57.72 ; 50.69 ; 8.66 ; 31.63 ; 38.6$;

잉 $\quad-7.43 ;-47.46 ;-39.49 ; 17.48 ; 11.45 ;-14.58 ;-34.61 ;-33.64 ; 60.33 ; 13.3 ; 10.27$;

응 $\quad-14.76 ; 17.21 ; 15.18 ; 5.15 ;-22.88 ;-37.91 ;-3.94 ; 10.03 ;-50 ; 21.97 ;-42.06 ;$

\% $\quad-5.72 ;-0.57 ;-13.42 ; 10.73 ; 2.88 ;-23.97 ;-15.82 ; 33.33 ; 42.48 ; 6.63 ; 3.78$;

잉 $\quad-9.07 ;-32.92 ; 0.23 ; 1.38]$;

ㅇemoved Trend with 4th-Order Polynomial Function:

을 $\mathrm{z} 10 \mathrm{R}=[16.16226914 ;-2.891364489 ;-3.340443671 ;-19.37100678 ;-6.163364854$;

응 $\quad-1.892101543 ; 7.273926843 ; 53.17159136 ;-8.356509593 ;-64.46205023$;

\% $\quad 20.70902256 ; 8.016489276 ; 4.32585776 ; 6.508363213 ;-3.559031801$;

잉 $\quad-2.993637363 ;-71.90703619 ; 36.59491634 ; 32.41209224 ;-6.549909138$;

\% $\quad 19.62023891 ; 29.83959046 ;-12.96907306 ;-49.87724286 ;-38.95068279$;

잉 $20.75057064 ; 17.17220831 ;-6.734351567 ;-25.01196341 ;-22.69775428$;

응 $\quad 72.17687613 ; 25.58625546 ; 22.51043873 ;-3.064791684 ; 27.85207395 ;$

잉 $\quad 24.25827271 ; 12.15676905 ;-18.44374521 ;-36.52885093 ;-6.078401567$;

\% $\quad 3.933476753 ;-60.46161473 ; 6.773652586 ;-62.31766534 ; 20.2348399$;

응 $\quad 20.40891767 ; 2.718167925 ; 22.2514655 ; 10.10341257 ;-20.62566132$;

응 $\quad-15.82969926 ; 30.60308299 ; 37.79019706 ; 0.85488192 ;-2.073896093$;

응 $\quad-13.86144329 ;-35.36733861 ; 1.554566346 ; 8.056147348]$;

\% $\mathrm{zlOR}=\mathrm{flipud}(\mathrm{z} 10 \mathrm{R}$ correct) $\%$ to check the reverse direction

$\circ 12 \mathrm{~m}$ Point values (not averaged):

ㅇ $\mathrm{z} 12 \mathrm{R}=[365 ; 375 ; 400 ; 345 ; 370 ; 380 ; 375 ; 410 ; 360 ; 380 ; 420 ; 435 ; 410 ; 395 ; 360 ; 380 ; 340$;

응 $\quad 365 ; 375 ; 395 ; 365 ; 340 ; 340 ; 340 ; 375 ; 365 ; 365 ; 330 ; 320 ; 350 ; 360 ; 0 ; 395 ; 305 ;$

응 $\quad 330 ; 340 ; 330 ; 335 ; 335 ; 365 ; 340 ; 310 ; 360 ; 340 ; 360 ; 365 ; 360 ; 385 ; 360 ; 365 ; 340$;

응 $\quad 480 ; 400 ; 395 ; 390 ; 350 ; 365 ; 375 ; 425]$;

을ese are the old $12 \mathrm{~m}$ phase velocity values:

$z 1201 d=[365 ; 395 ; 400 ; 360 ; 395 ; 365 ; 375 ; 400 ; 390 ; 380 ; 420 ; 420 ; 420 ; 420 ; 380 ; 390 ; 355 ; 380$; 
응 $\quad 390 ; 375 ; 415 ; 305 ; 345 ; 360 ; 380 ; 380 ; 380 ; 330 ; 335 ; 365 ; 355 ; 0 ; 390 ; 315 ; 355 ; 355 ; 355 ;$ $\quad 340 ; 345 ; 325 ; 310 ; 330 ; 375 ; 360 ; 380 ; 380 ; 380 ; 400 ; 405 ; 395 ; 335 ; 400 ; 420 ; 420 ; 410$; 잉 $355 ; 385 ; 385 ; 335]$;

\%The zloS-values are the Shear wave velocities at 10 meters depth; they are opulled from the shear wave velocity model given after inversion.

oNote that values of "O" are not the actual phase velocities; this means othat the location was not able to be measured. The code will delete these ovalues automatically.

ㅇill adjusted:

$\mathrm{z10S}=[302 ; 334 ; 337 ; 350 ; 312 ; 310 ; 334 ; 376 ; 345 ; 329 ; 368 ; 385 ; 346 ; 375 ; 363 ; 343 ; 283$;

$368 ; 353 ; 350 ; 355 ; 328 ; 298 ; 289 ; 326 ; 332 ; 326 ; 311 ; 294 ; 281 ; 319 ; 328 ; 346 ; 292$;

$293 ; 296 ; 307 ; 288 ; 297 ; 325 ; 289 ; 274 ; 331 ; 297 ; 371 ; 375 ; 375 ; 401 ; 386 ; 368 ; 334$;

$454 ; 397 ; 399 ; 372 ; 353 ; 353 ; 378 ; 408]$;

№n-hill adjusted:

$\mathrm{z} 10 \mathrm{~S}=[302 ; 334 ; 337 ; 350 ; 312 ; 310 ; 334 ; 376 ; 345 ; 329 ; 368 ; 385 ; 346 ; 375 ; 363 ; 343 ; 283$;

응 $\quad 368 ; 353 ; 350 ; 355 ; 328 ; 298 ; 289 ; 326 ; 332 ; 326 ; 311 ; 294 ; 281 ; 319 ; 328 ; 346 ; 292$;

음 $\quad 293 ; 296 ; 307 ; 288 ; 297 ; 325 ; 289 ; 274 ; 331 ; 297 ; 348 ; 355 ; 345 ; 377 ; 367 ; 346 ; 311$;

\% $\quad 423 ; 386 ; 369 ; 348 ; 337 ; 328 ; 350 ; 369]$;

Removed Trend with Linear Piecewise Function:

\% $\mathrm{z} 10 \mathrm{~S}=[-48.374 ;-15.178 ;-10.982 ; 3.214 ;-33.59 ;-34.394 ;-9.198 ; 33.998 ; 4.194$;

ㅇ $\quad-10.61 ; 29.586 ; 47.782 ; 9.978 ; 40.174 ; 29.37 ; 10.566 ;-48.238 ; 37.958 ; 24.154 ;$

응 $\quad 22.35 ; 28.546 ; 2.742 ;-26.062 ;-33.866 ; 4.33 ; 11.526 ; 6.722 ;-7.082 ;-22.886$;

\% $\quad-34.69 ; 4.506 ; 14.702 ; 33.898 ;-18.906 ;-16.71 ;-12.514 ;-0.318 ;-18.122$;

응 $\quad-7.926 ; 21.27 ;-13.534 ;-27.338 ; 30.858 ;-1.946 ;-32.148 ;-25.355 ;-35.562$;

응 $\quad-3.769 ;-13.976 ;-35.183 ;-70.39 ; 41.403 ; 4.196 ;-13.011 ;-34.218 ;-45.425$;

\% $\quad-54.632 ;-32.839 ;-14.046]$;

을

$\mathrm{z} 10 \mathrm{~S}=[0.452582866 ; 20.01986364 ; 12.46899293 ; 16.6599005 ;-28.54307087$;

응 $\quad-36.2711654 ;-16.65121432 ; 22.19436414 ;-10.85243524 ;-27.90520468$;

$\quad 10.92687659 ; 28.53904234 ;-9.16906065 ; 21.7066274 ; 12.07457925 ;$

응 $\quad-5.152319304 ;-62.0567695 ; 26.28294044 ; 14.79293528 ; 15.40375281$;

응 $\quad 24.05034381 ; 0.672072033 ;-25.78728573 ;-31.37953971 ; 8.847912874$;

응 $\quad 17.85208779 ; 14.59441382 ; 2.04073273 ;-12.8387007 ;-24.06921869 ;$

\% $\quad 15.32825953 ; 25.33722773 ; 43.94559269 ;-9.854325813 ;-9.065795006$;

응 $\quad-6.687669114 ; 3.285610638 ;-17.13598397 ;-9.938068178 ; 15.8981558$;

잉 $\quad-22.60410125 ;-40.41721558 ; 13.49084961 ;-23.84345592 ; 8.434107842$;

응 $\quad 11.6581513 ;-1.981463534 ; 26.58701712 ; 13.43976003 ;-10.34265502$;

잉 $\quad-47.67523527 ; 62.53142508 ; 24.37114478 ; 6.942155615 ;-13.65289764$;

응 $\quad-23.30695721 ;-29.90855231 ;-4.341799187 ; 19.51359895]$;

\%The z15R-values are the Rayleigh wave velocities at 15 meters depth; note othat they are just "eye-balled" values from the HV curve.

oNote that values of "0" are not the actual phase velocities; this means othat the location was not able to be measured. The code will delete these ovalues automatically.

№n-Hill adjusted:

$\mathrm{z} 15 \mathrm{R}=[245 ; 250 ; 268 ; 254 ; 278 ; 289 ; 306 ; 356 ; 306 ; 258 ; 338 ; 329 ; 324 ; 319 ; 312 ; 307 ; 239$; $337 ; 326 ; 297 ; 308 ; 0 ; 268 ; 236 ; 245 ; 292 ; 286 ; 257 ; 242 ; 246 ; 321 ; 0 ; 0 ; 0 ; 277 ; 278$; $269 ; 244 ; 230 ; 0 ; 0 ; 214 ; 281 ; 221 ; 318 ; 321 ; 315 ; 335 ; 328 ; 303 ; 302 ; 0 ; 360 ; 335$; 
$329 ; 310 ; 295 ; 325 ; 0] ;$

Removed Trend with Linear Piecewise Function:

$\mathrm{z} 15 \mathrm{R}=[-57.476 ;-51.3545 ;-32.233 ;-45.1115 ;-19.99 ;-7.8685 ; 10.253 ; 61.3745$;

응 $\quad 12.496 ;-34.3825 ; 46.739 ; 38.8605 ; 34.982 ; 31.1035 ; 25.225 ; 21.3465 ;-45.532$;

잉 $\quad 53.5895 ; 43.711 ; 15.8325 ; 27.954 ; 0 ;-9.803 ;-40.6815 ;-30.56 ; 17.5615 ; 12.683$;

응 $\quad-15.1955 ;-29.074 ;-23.9525 ; 52.169 ; 0 ; 0 ; 0 ; 12.655 ; 14.7765 ; 6.898 ;-16.9805$;

\% $\quad-29.859 ; 0 ; 0 ;-42.4945 ; 25.627 ;-33.2515 ;-4.03 ;-0.905 ;-6.78 ; 13.345 ; 6.47$;

\% $\quad-18.405 ;-19.28 ; 0 ; 38.97 ; 14.095 ; 8.22 ;-10.655 ;-25.53 ; 4.595 ; 0]$;

Removed Trend with 4th-Order Polynomial Function:

일 $\mathrm{z}=[14.22515578 ; 1.354720969 ; 4.011223226 ;-22.98865739 ;-9.82253464$;

$\quad-7.662316129 ; 2.325796718 ; 46.98130865 ;-6.850569429 ;-57.31892042$;

응 $\quad 21.43287893 ; 12.26715804 ; 8.051952528 ; 4.661004154 ;-0.026239139$;

\% $\quad-2.124623237 ;-66.74328786 ; 35.01433346 ; 28.05051333 ; 3.273230568$;

응 $18.59617013 ; 0 ;-12.77401304 ;-40.61123498 ;-27.63643301 ; 23.0926087$;

$\quad 20.52381213 ;-5.389194533 ;-17.68707697 ;-11.40479466 ; 65.42839907 ; 0 ; 0 ; 0$;

잉 $\quad 23.88493777 ; 24.23360479 ; 14.07749289 ;-12.57270804 ;-28.70060195 ; 0 ; 0$;

잉 $\quad-53.70088856 ; 9.538529485 ;-54.53217265 ; 19.43438762 ; 17.76597497$;

ㄱ $\quad 7.261703262 ; 23.01214876 ; 12.11359391 ;-16.33197268 ;-20.21685621 ; 0$;

응 $\quad 34.15473588 ; 8.655131925 ; 3.204051876 ;-14.06227863 ;-26.00192778$;

\% $8.532742417 ; 0]$;

15m Point values (not averaged):

$\mathrm{z} 15 \mathrm{R}=[425 ; 415 ; 445 ; 365 ; 410 ; 415 ; 410 ; 435 ; 400 ; 405 ; 465 ; 480 ; 445 ; 425 ; 395 ; 415 ; 385$;

잉 $\quad 390 ; 415 ; 440 ; 390 ; 0 ; 365 ; 380 ; 420 ; 405 ; 410 ; 365 ; 365 ; 400 ; 410 ; 0 ; 0 ; 0$;

응 $\quad 360 ; 380 ; 390 ; 380 ; 395 ; 0 ; 0 ; 360 ; 415 ; 370 ; 395 ; 395 ; 415 ; 410 ; 385 ; 400 ; 375$;

응 $\quad 0 ; 430 ; 435 ; 430 ; 375 ; 410 ; 420 ; 0]$;

\%These are the old $15 \mathrm{~m}$ phase velocity values:

$\mathrm{z} 150 \mathrm{ld}=[455 ; 435 ; 440 ; 365 ; 430 ; 405 ; 410 ; 430 ; 415 ; 405 ; 440 ; 440 ; 445 ; 440 ; 410 ; 420 ; 400$;

$\quad 400 ; 390 ; 415 ; 445 ; 0 ; 360 ; 380 ; 425 ; 420 ; 420 ; 370 ; 380 ; 415 ; 0 ; 0 ; 0 ; 0$;

응 $\quad 380 ; 395 ; 400 ; 375 ; 380 ; 380 ; 0 ; 365 ; 425 ; 385 ; 405 ; 405 ; 415 ; 440 ; 415 ; 410 ; 355$;

응 $\quad 400 ; 435 ; 465 ; 455 ; 385 ; 0 ; 435 ; 0]$;

\%The z15S-values are the Shear wave velocities at 15 meters depth; they are opulled from the shear wave velocity model given after inversion.

oNote that values of "O" are not the actual phase velocities; this means

othat the location was not able to be measured. The code will delete these \%values automatically.

\%Hill adjusted:

$\mathrm{z} 15 \mathrm{~S}=[384 ; 422 ; 429 ; 360 ; 367 ; 383 ; 377 ; 424 ; 395 ; 378 ; 418 ; 429 ; 415 ; 415 ; 412 ; 399 ; 354$;

$418 ; 407 ; 417 ; 391 ; 0 ; 346 ; 364 ; 404 ; 404 ; 392 ; 347 ; 376 ; 362 ; 388 ; 0 ; 0 ; 0 ;$

$345 ; 373 ; 369 ; 345 ; 362 ; 0 ; 0 ; 334 ; 386 ; 367 ; 410 ; 408 ; 429 ; 442 ; 418 ; 404 ; 374 ;$

$0 ; 422 ; 455 ; 422 ; 379 ; 404 ; 429 ; 0]$;

№n-hill adjusted:

$\mathrm{z} 15 \mathrm{~S}=[384 ; 422 ; 429 ; 360 ; 367 ; 383 ; 377 ; 424 ; 395 ; 378 ; 418 ; 429 ; 415 ; 415 ; 412 ; 399 ; 354$;

응 $\quad 418 ; 407 ; 417 ; 391 ; 0 ; 346 ; 364 ; 404 ; 404 ; 392 ; 347 ; 376 ; 362 ; 388 ; 0 ; 0 ; 0$;

응 $\quad 345 ; 373 ; 369 ; 345 ; 362 ; 0 ; 0 ; 334 ; 386 ; 367 ; 396 ; 397 ; 410 ; 429 ; 407 ; 392 ; 360 ; 0$;

능 $\quad 408 ; 435 ; 400 ; 370 ; 386 ; 412 ; 0]$;

ㅇemoved Trend with Linear Piecewise Function:

$\mathrm{z} 15 \mathrm{~S}=[-23.526 ; 15.578 ; 23.682 ;-44.214 ;-36.11 ;-19.006 ;-23.902 ; 24.202 ;-3.694$;

잉 $\quad-19.59 ; 21.514 ; 33.618 ; 20.722 ; 21.826 ; 19.93 ; 8.034 ;-35.862 ; 29.242 ; 19.346$;

응 $\quad 30.45 ; 5.554 ; 0 ;-37.238 ;-18.134 ; 22.97 ; 24.074 ; 13.178 ;-30.718 ;-0.614$; 
응 $\quad-13.51 ; 13.594 ; 0 ; 0 ; 0 ;-24.99 ; 4.114 ; 1.218 ;-21.678 ;-3.574 ; 0 ; 0 ;-28.262$;

응 $\quad 24.842 ; 6.946 ;-20.588 ;-19.3525 ;-6.117 ; 13.1185 ;-8.646 ;-23.4105 ;-55.175 ; 0$;

ㅇ $\quad-6.704 ; 20.5315 ;-14.233 ;-43.9975 ;-27.762 ;-1.5265 ; 0]$;

Removed Trend with 4th-Order Polynomial Function:

\% $\mathrm{z} 15 \mathrm{~S}=[4.134949991 ; 35.50529324 ; 36.95500408 ;-36.59690443 ;-33.22887974$;

응 $\quad-20.01682981 ;-28.03412309 ; 17.64841148 ;-12.03751556 ;-29.15765415$;

응 $\quad 11.22478526 ; 23.0491317 ; 10.25725375 ; 11.79355944 ; 10.60499632$;

응 $\quad-0.358948549 ;-43.14624863 ; 23.19766214 ; 14.62988931 ; 27.11007792$;

응 $\quad 3.600412521 ; 0 ;-36.52704459 ;-16.2077697 ; 25.99578439 ; 28.05849972$;

잉 $\quad 17.95779784 ;-25.32636019 ; 5.188526173 ;-7.512503018 ; 19.55813179 ; 0 ; 0 ; 0$;

$\quad-21.60221555 ; 6.229314981 ; 1.811316305 ;-22.85085553 ;-6.749304962 ; 0 ; 0$;

잉 $\quad-38.7452709 ; 11.54091474 ;-9.331606967 ; 8.823695903 ; 7.573596112$;

\% $\quad 18.38004632 ; 35.28395556 ; 11.32877241 ;-5.439515102 ;-38.97237942 ; 0$;

읭 $\quad 6.874969235 ; 33.3649348 ;-1.690171232 ;-31.22912382 ;-14.18815842$;

잉 $\quad 13.49902903 ; 0]$;

The z20S-values are the Shear wave velocities at 20 meters depth; they are opulled from the shear wave velocity model given after inversion.

№te that values of "O" are not the actual phase velocities; this means

othat the location was not able to be measured. The code will delete these \%values automatically.

\%Hill adjusted:

$\mathrm{z} 20 \mathrm{~S}=[0 ; 0 ; 0 ; 364 ; 434 ; 443 ; 429 ; 454 ; 424 ; 410 ; 449 ; 452 ; 0 ; 438 ; 436 ; 434 ; 0 ;$

$449 ; 0 ; 0 ; 411 ; 0 ; 0 ; 0 ; 459 ; 453 ; 436 ; 0 ; 0 ; 0 ; 0 ; 0 ; 0 ; 0 ;$

$378 ; 429 ; 410 ; 0 ; 0 ; 0 ; 0 ; 0 ; 0 ; 0 ; 0 ; 438 ; 0 ; 469 ; 0 ; 0 ; 0$;

$0 ; 0 ; 0 ; 0 ; 0 ; 0 ; 0 ; 0]$;

\%Non-hill adjusted:

z $20 S=[0 ; 0 ; 0 ; 364 ; 434 ; 443 ; 429 ; 454 ; 424 ; 410 ; 449 ; 452 ; 0 ; 438 ; 436 ; 434 ; 0 ;$

\% $449 ; 0 ; 0 ; 411 ; 0 ; 0 ; 0 ; 459 ; 453 ; 436 ; 0 ; 0 ; 0 ; 0 ; 0 ; 0 ; 0$;

응 $378 ; 429 ; 410 ; 0 ; 0 ; 0 ; 0 ; 0 ; 0 ; 0 ; 426 ; 427 ; 0 ; 460 ; 0 ; 424 ; 0 ; 0 ; 451 ; 0 ; 454$;

응 $0 ; 0 ; 0 ; 0]$;

ochoosing which z-values to use

if indicate_wave_type $==1$

if indicate_depth==5

$\mathrm{z}=\mathrm{z} 05 \mathrm{R}$;

else

if indicate_depth $==10$

$\mathrm{z}=\mathrm{z} 10 \mathrm{R}$;

else

if indicate_depth $==15$

$z=z 15 R$;

else

fprintf('Error: the depth needs to be at 5, 10, or 15 meters. $\backslash n^{\prime}$ ) end

end

end

else

if indicate_wave_type $==2$

if indicate_depth==5 


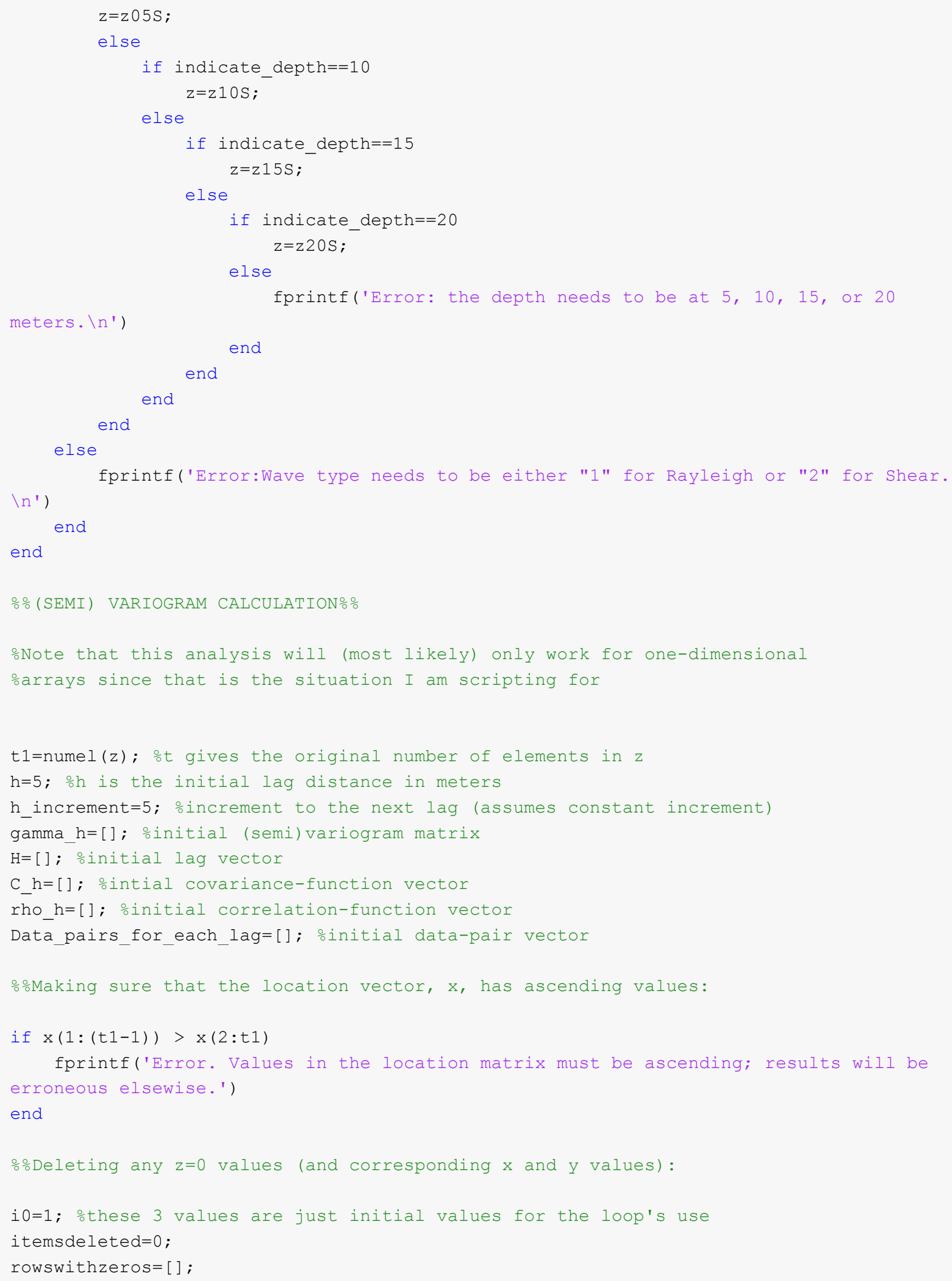




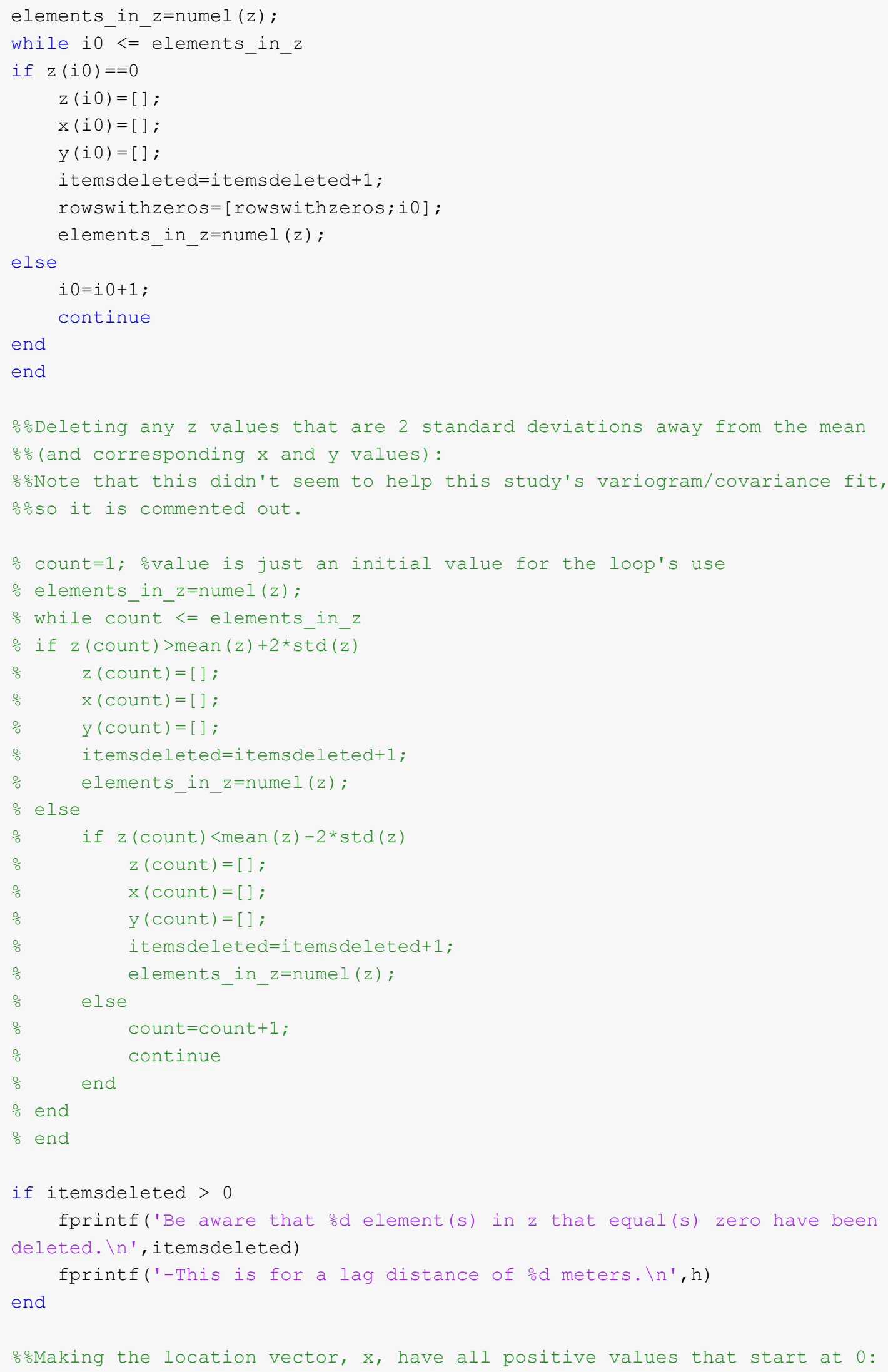




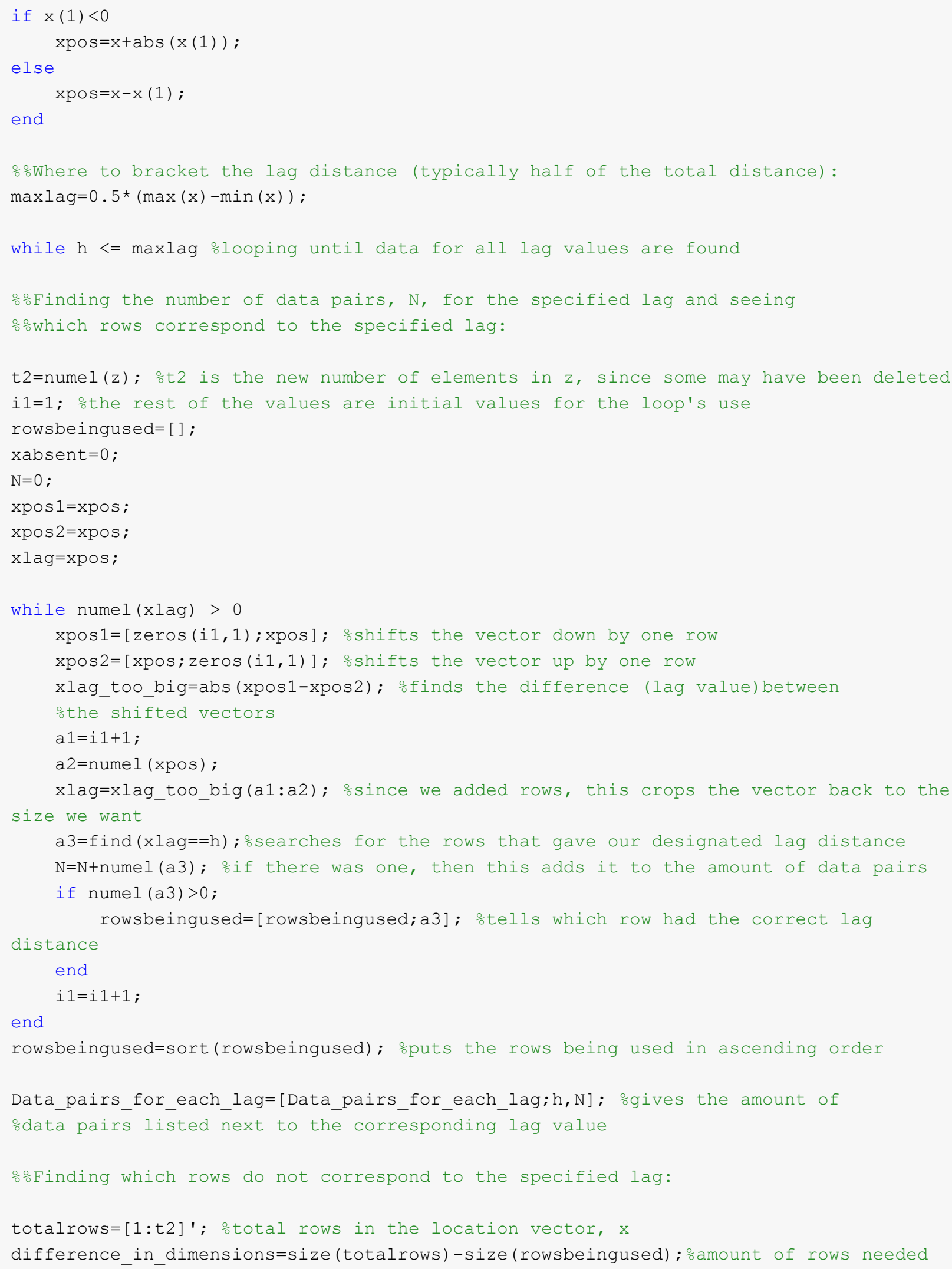




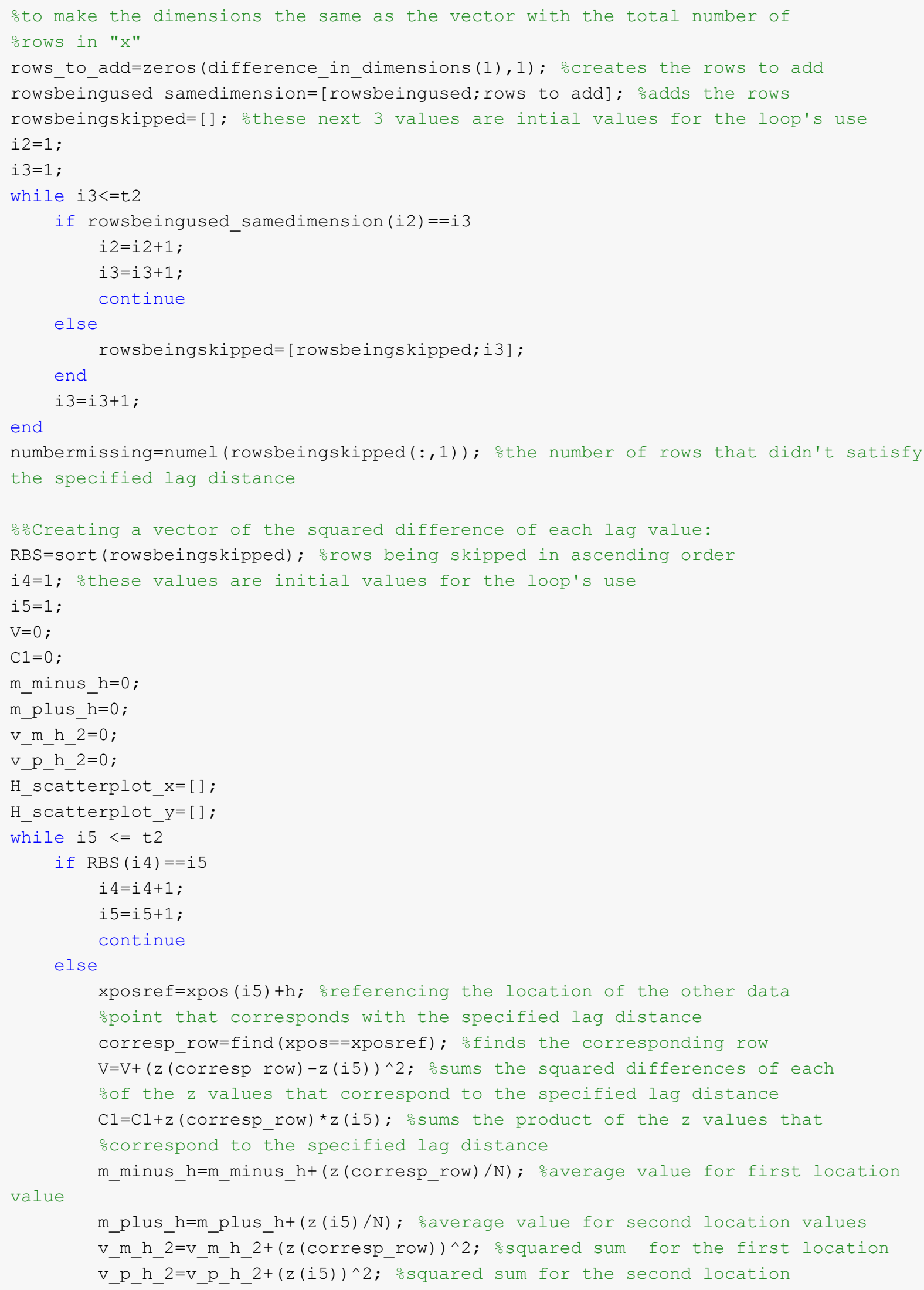


end

i $5=i 5+1$;

end

을eating the semivariogram point corresponding to the specified lag by

\%ㅇviding by the number of data pairs and halving the value:

$\mathrm{H}=[\mathrm{H} ; \mathrm{h}]$; \%creating lag vector

gamma_h=[gamma_h;V/ $(2 * N)]$; ocreating (semi) variogram vector

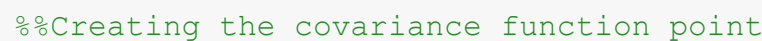

C_h $=\left[C_{-} h ;\left(\mathrm{C} 1-\mathrm{N}^{*}\left(\mathrm{~m} \_\right.\right.\right.$minus_h*m_plus_h $\left.\left.)\right) / \mathrm{N}\right]$; ocreating the covariance function vector oNotice that the multiplication of the mean values is multiplied by the onumber of pairs. This is because they are a constant value subtracted from \%the equation $\mathrm{N}$ amount of times.

응 Creating the correlation function point

sigma_minus_h=sqrt ( (v_m_h_2/N)-(m_minus_h $\left.)^{\wedge} 2\right)$; \%standard deviation for the first location

sigma_plus_h=sqrt ( (v_p_h_2/N)-(m_plus_h)^2); \%standard deviation for the second location

rho_h=[rho_h; ( $\mathrm{Cl}-\mathrm{N}^{*}\left(\mathrm{~m} \_\right.$minus_h*m_plus_h $\left.\left.)\right) / \mathrm{N}\right) /($ sigma_minus_h*sigma_plus_h $\left.)\right]$; \%creating othe correlation function vector

$\mathrm{h}=\mathrm{h}+\mathrm{h}$ _increment; $\%$ proceeding to the next lag value end

-Amount of data pairs

display(Data_pairs_for_each_lag)

\%semivariogram values:

semivariogram_data=table (H,gamma_h)

figure ocreate new figure

subplot $(1,2,1)$

plot (H, gamma_h, 'ko-')

title(' (Semi)Variogram')

xlabel('h, lag distance')

ylabel (' 'gamma (h) ')

ocovariance function:

covariance_function_data=table (H,C_h)

subplot $(1,2,2)$

plot $\left(\mathrm{H}, \mathrm{C} \_\mathrm{h}, \mathrm{h}^{\mathrm{b}} \mathrm{o-}\right.$ ')

title('Covariance Function')

xlabel('h, lag distance')

ylabel ('C (h) ') 


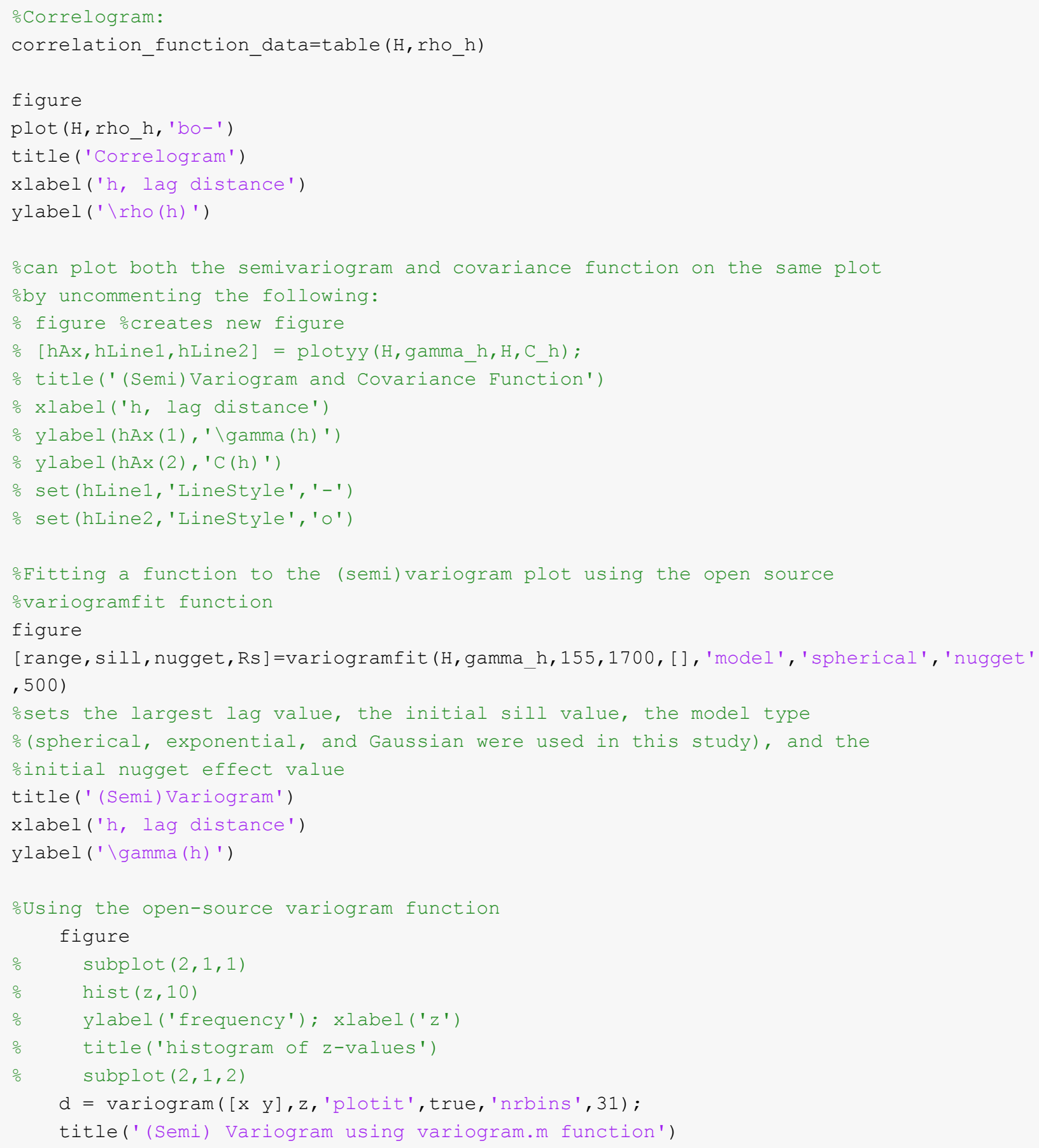

